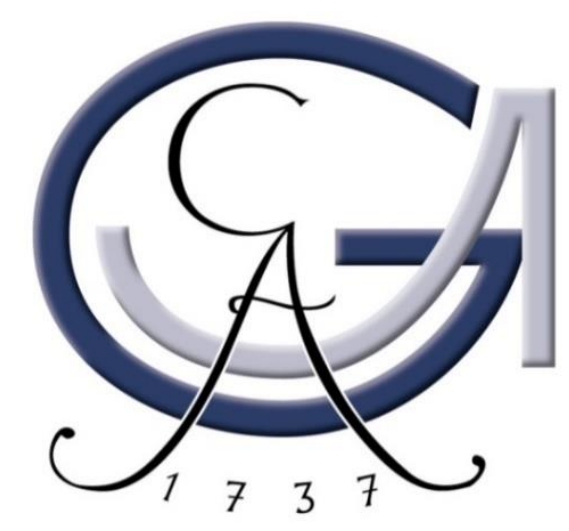

\title{
The influence of valproic acid and the role of cyclin D2 in prostate cancer
}

\author{
Doctoral Thesis \\ In partial fulfillment of the requirements for the degree \\ "Doctor rerum naturalium (Dr. rer. nat.)" \\ in the Molecular Medicine Study Program \\ at the Georg-August University Göttingen)
}

submitted by

Claudia Morich

born in Osterode am Harz, Germany

Göttingen, 2016 
Supervisor:

Dr. Silke Kaulfuß, Department of Human Genetics, University Medical Center Göttingen

Members of the Thesis Committee:

Official Supervisor:

Prof. Dr. Peter Burfeind, Department of Human Genetics, University Medical Center Göttingen

Second member of the thesis committee:

Prof. Dr. Matthias Dobbelstein, Department of Molecular Oncology, University Medical Center Göttingen

Third member of the thesis committee:

Prof. Dr. Dieter Kube, Department of Hematology and Oncology, University Medical Center Göttingen

Date of Disputation: 


\section{AFFIDAVIT}

Herewith I declare that my doctoral thesis entitled "The influence of valproic acid and the role of cyclin D2 in prostate cancer" has been written independently with no other sources and aids than quoted.

Göttingen, February 2016

Claudia Morich 


\section{Table of Contents}

List of Abbreviations .................................................................................... VI

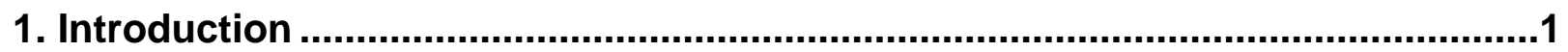

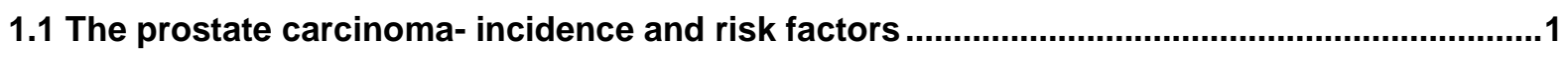

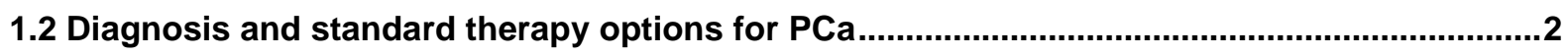

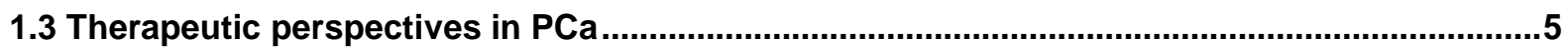

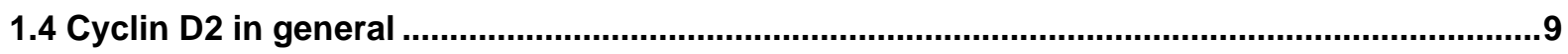

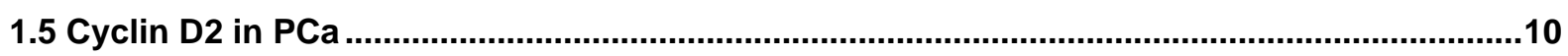

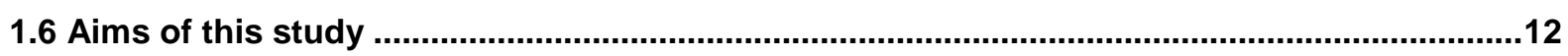

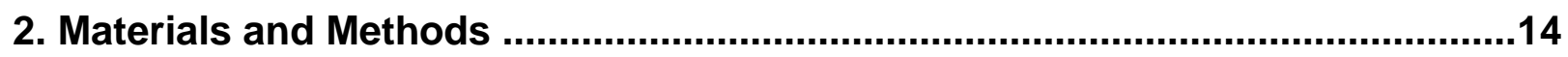

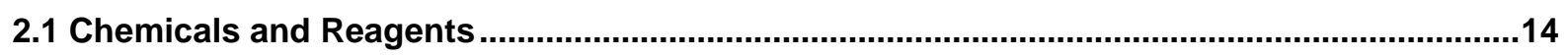

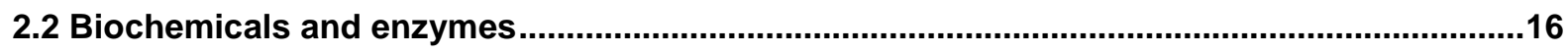

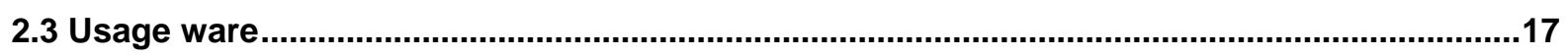

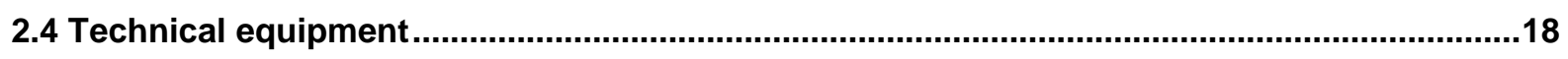

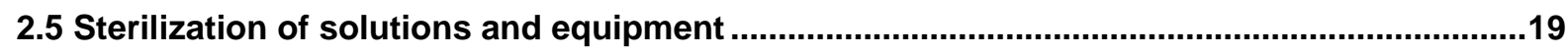

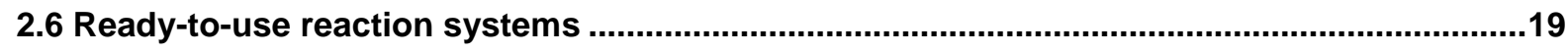

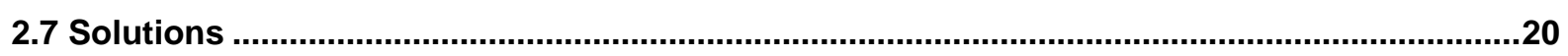

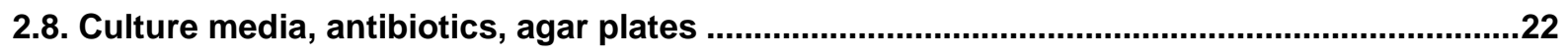

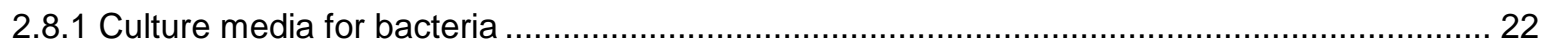

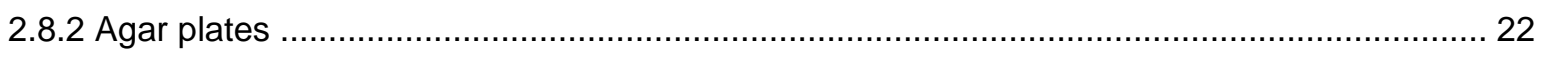

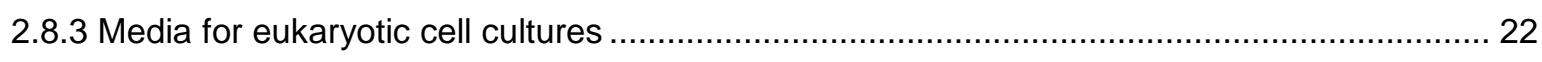

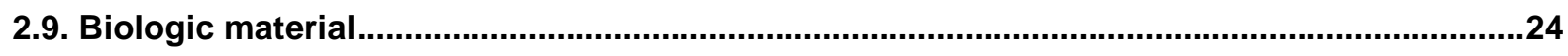

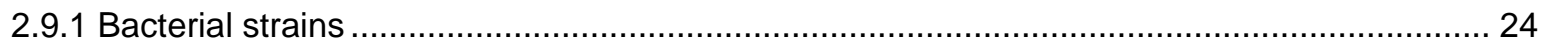

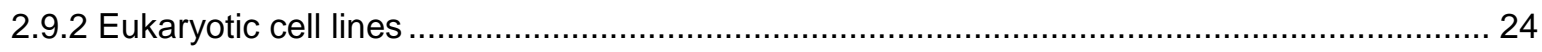

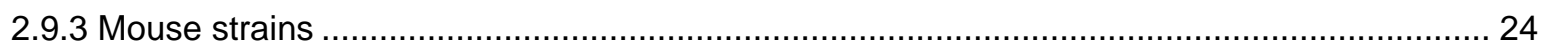

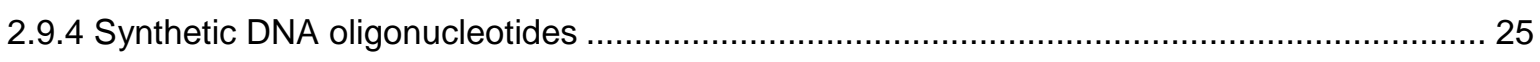

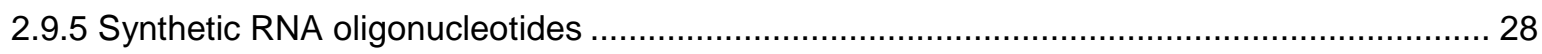

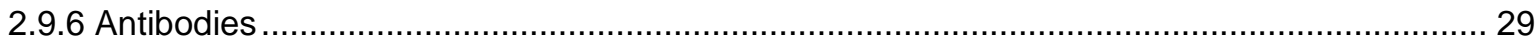

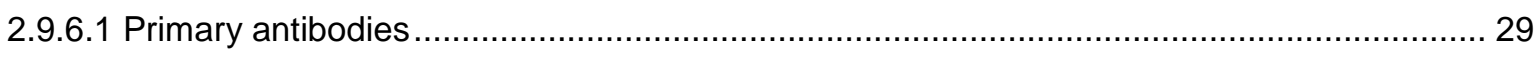

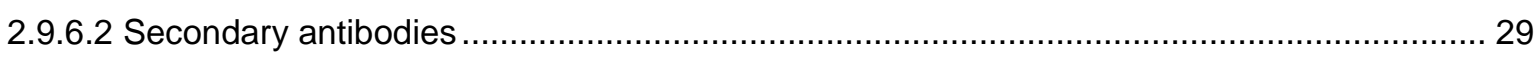

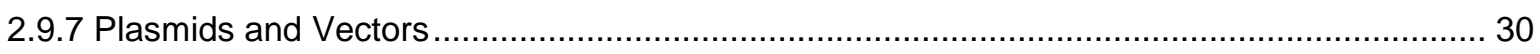




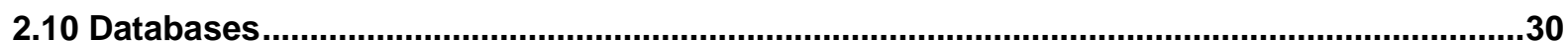

2.11 Isolation, purification and concentration determination of nucleic acids...........................31

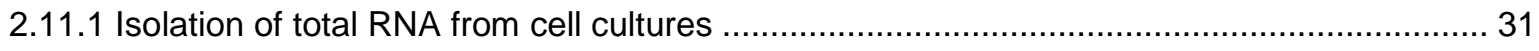

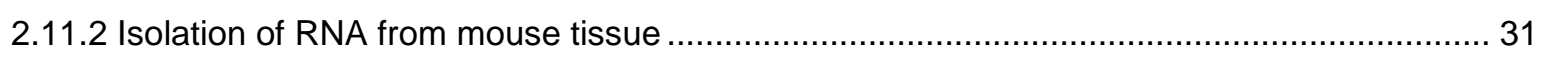

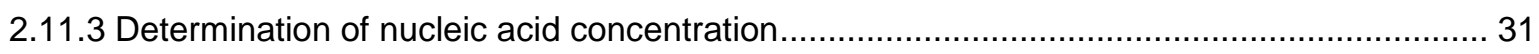

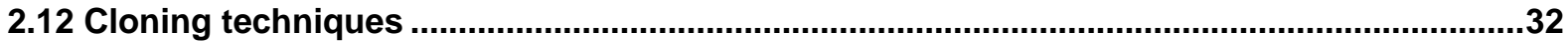

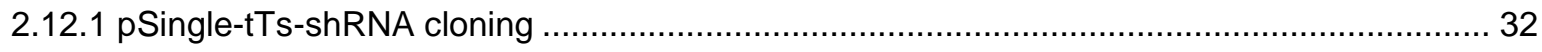

2.12.2 Amplification of DNA for subcloning into pGEM®-T Easy vector ....................................... 32

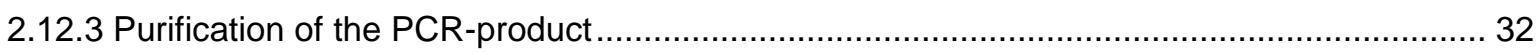

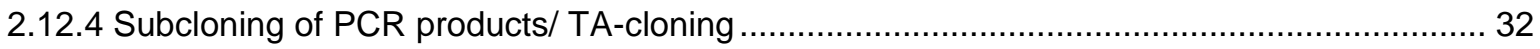

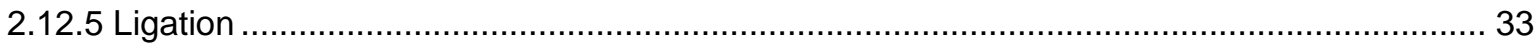

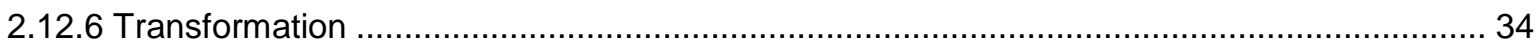

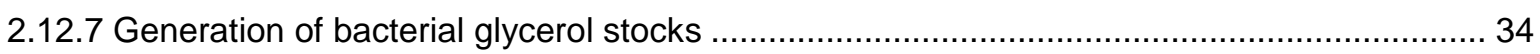

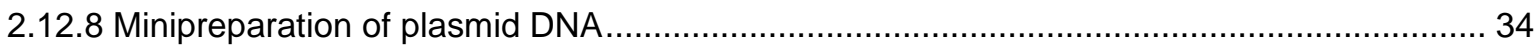

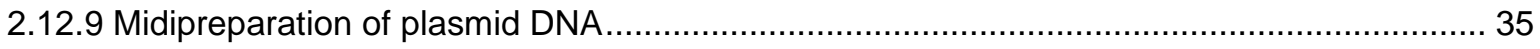

2.12.10 Cleavage of DNA with restriction endonucleases ...................................................... 35

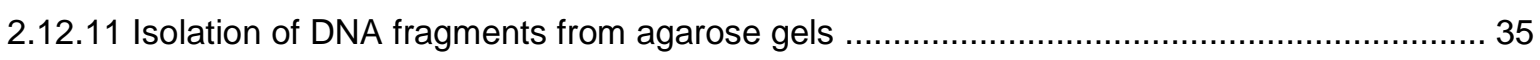

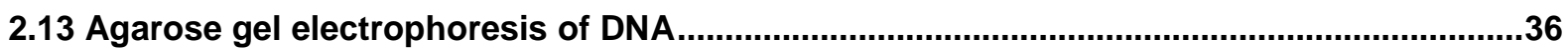

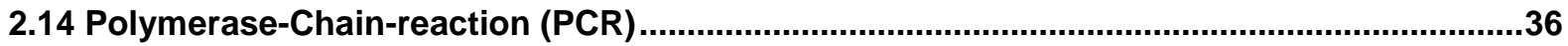

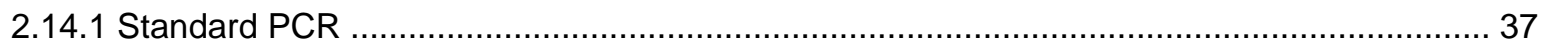

2.14.2 Amplification of DNA fragments for molecular cloning/ Touchdown PCR .......................... 37

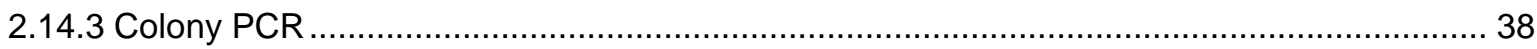

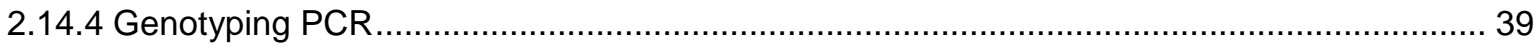

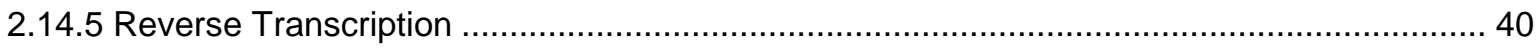

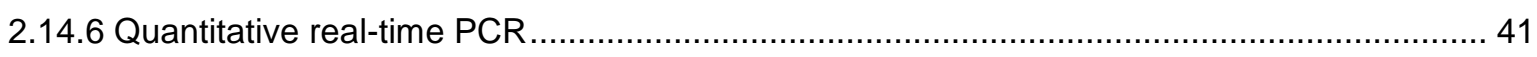

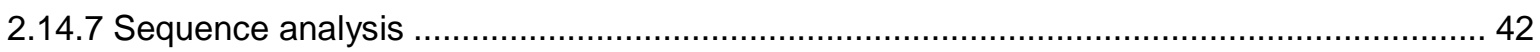

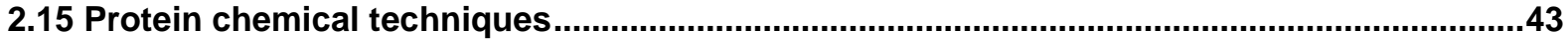

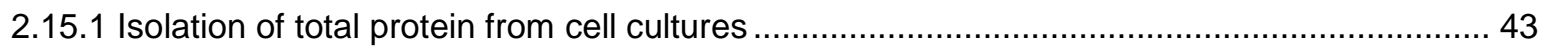

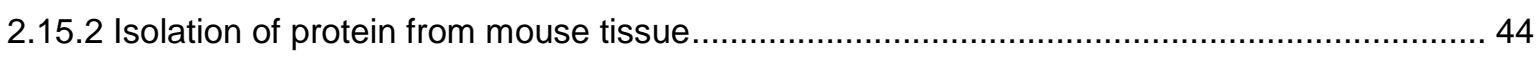

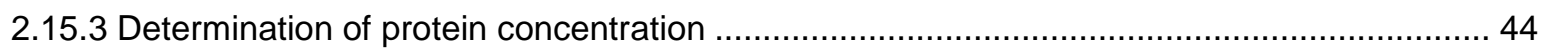

2.15.4 Sodium Dodecyl Sulfate Polyacrylamide gel electrophoresis (SDS-PAGE) ...................... 45

2.15.5 Transfer of proteins onto a PVDF membrane .............................................................. 45

2.15.6 Incubation of protein-bound membranes with antibodies.............................................. 46

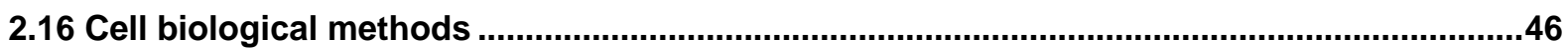

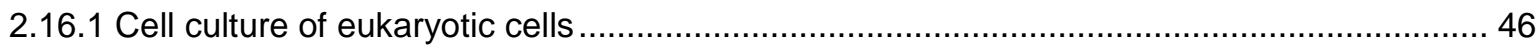

2.16.2 Cryo-preservation and revitalization of eukaryotic cells .................................................. 46 


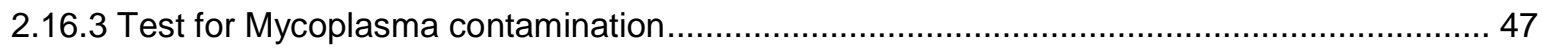

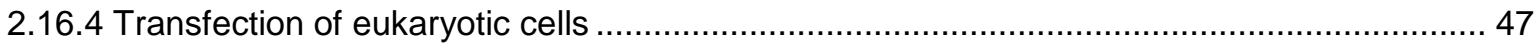

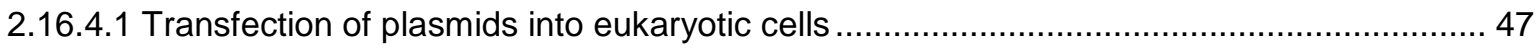

2.16.4.1.1 Generation of single-cell clones/populations .......................................................... 48

2.16.4.2 Transfection of small interfering RNA (siRNA) into eukaryotic cells ................................ 49

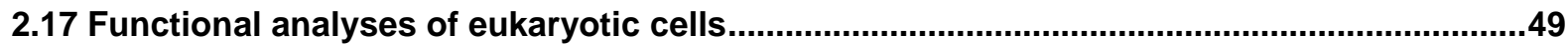

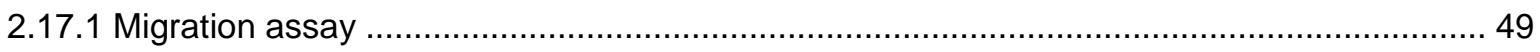

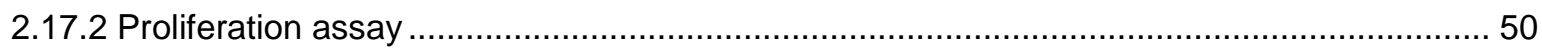

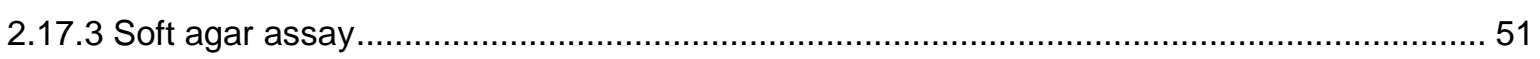

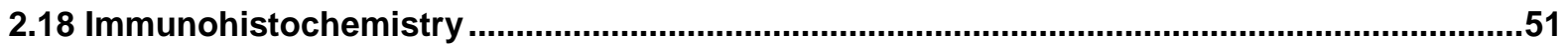

2.18 .1 Immunofluorescent staining of eukaryotic cells ......................................................... 51

2.18.2 Immunohistochemical staining of cryo-sections from CAM tumors .................................. 52

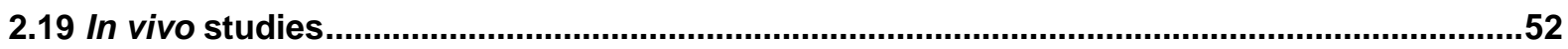

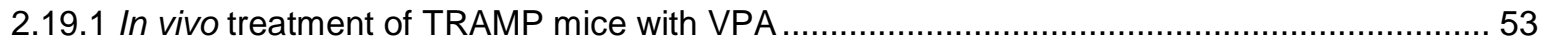

2.19.2 Chicken chorioallantoic membrane (CAM) assay .......................................................... 53

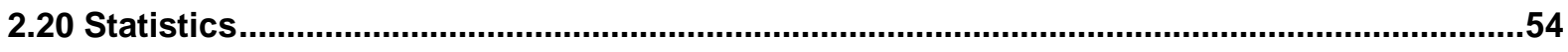

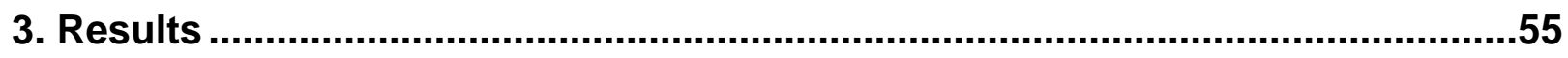

3.1 Molecular effects of VPA treatment in PCa therapy ................................................................55

3.1.1 Verification of deregulated candidate gene expression in tumors of VPA-treated TRAMP mice

3.1.1.1 Verification of deregulated candidate gene expression on RNA and protein level ............. 56

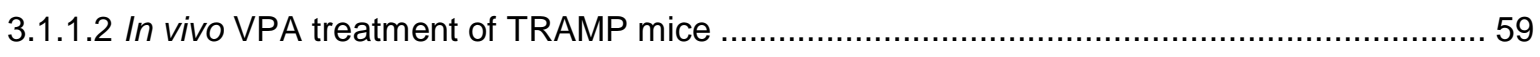

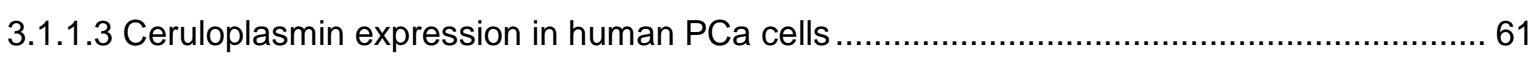

3.1.2 Effects of VPA treatment on the expression of angiogenesis markers ................................ 63

3.1.2.1 Effect of in vitro VPA treatment on the expression of blood- and lymphangiogenesis markers 63

3.1.2.1.1 Is the Vegfr2 signaling pathway activated by VPA treatment? ....................................... 68

3.1.2.2 Effect of in vivo VPA treatment on the expression of blood- and lymphangiogenesis markers 69

3.1.2.3 Studying the influence of VPA on angiogenesis in vivo - CAM experiments .................... 72

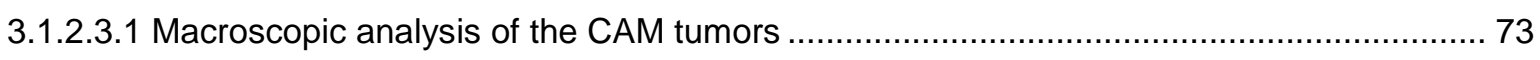

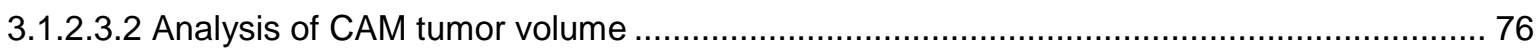

3.1.2.3.3 Studying blood and lymphangiogenesis in the CAM tumors with the markers Mep21 and

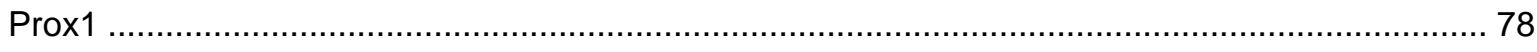

3.1.2.3.4 Expression analysis of angiogenesis markers in CAM tumors ................................... 80

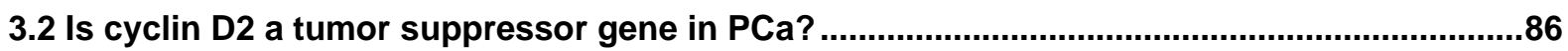


3.2.1 Cyclin D2 overexpression studies in human PCa cells 86

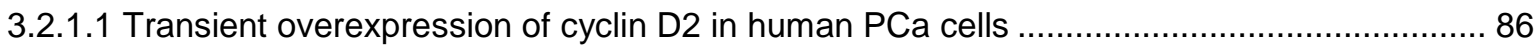

3.2.1.1.1 Generation of the pIRES2-EGFP-CCND2 expression vector ..................................... 86

3.2.1.1.2 Studies on the proliferation rate of PCa cells with transient cyclin D2 over-expression ... 88

3.2.1.2 Stable overexpression of cyclin D2 in human PCa cells ................................................ 89

3.2.1.2.1 Generation of the pEBTetD-CCND2 expression vector ............................................. 89

3.2.1.2.2 Functional effects of stable and inducible cyclin D2 overexpression .............................. 95

3.2.1.2.2.1 Studies on the proliferation rate of PCa cells with stable or inducible cyclin D2 overexpression

3.2.1.2.2.2 Studies on the migration rate of PCa cells with stable or inducible cyclin D2 overexpression 97

3.2.2 Cyclin D2 downregulation studies in $\mathrm{NIH} / 3 \mathrm{~T} 3$ cells ...................................................... 98

3.2.2.1 Influence of reduced cyclin D2 expression on migration behavior of NIH/3T3 cells 98

3.2.2.2 Influence of siRNA-mediated reduced cyclin D2 expression on transformation potential of $\mathrm{NIH} / 3 \mathrm{~T} 3$ cells 101

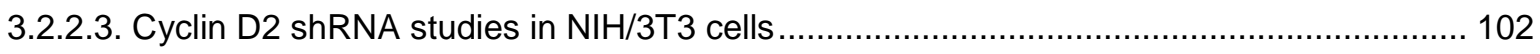

3.2.2.3.1 Generation of $\mathrm{NIH} / 3 \mathrm{~T} 3$ cell clones with a stable cyclin D2 downregulation ...... 102

3.2.2.3.2 Studies on the proliferation rate of $\mathrm{NIH} / 3 \mathrm{~T} 3$ cells with shRNA-mediated cyclin D2 downregulation 104

3.2.2.3.3.3 Influence of shRNA-mediated reduced expression of cyclin D2 on the transformation potential of $\mathrm{NIH} / 3 \mathrm{~T} 3$ cells

3.2.3 Studies on the functional consequences of cyclin D2 downregulation in VPA- treated PCa cells. 106

3.2.4 Generation of a conditional cyclin D2 knockout mouse model......................................... 109

3.2.4.1 Establishment of the Ccnd $2^{f / f f l}$ and $P B-C r e 4^{+}$mouse lines ............................................ 109

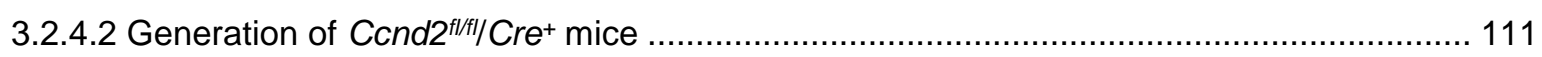

3.2.4.3 Verification of the prostate-specific cyclin D2 knockout .............................................. 113

3.2.4.4 Mating of the putative conditional cyclin D2 knockout mice with TRAMP mice ............... 116

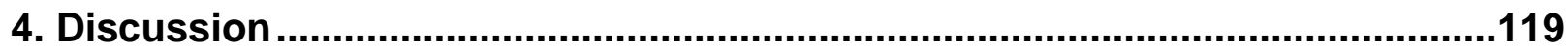

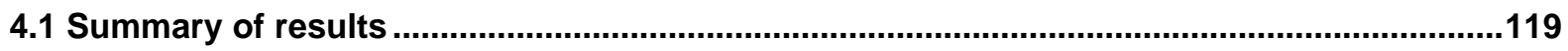

4.2 The histone deacetylase inhibitor VPA ...................................................................................122

4.2.1 Deregulated candidate genes after VPA treatment of mouse 2E PCa cells ....................... 126

4.2.1.1 Candidate gene expression in prostate tumor tissue and prostate tissue of in vivo VPA-

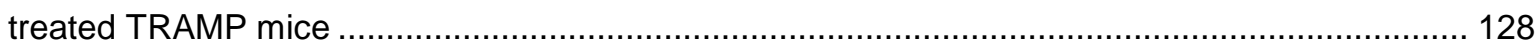

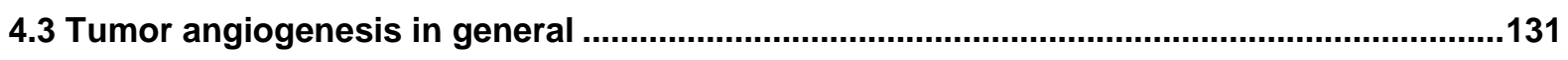

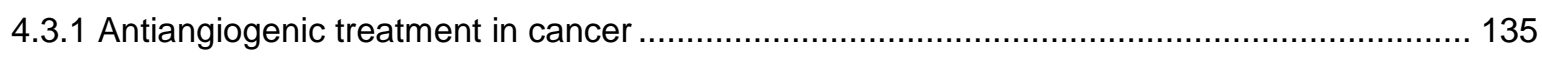

4.3.1.1 VPA as a possible anti-angiogenic treatment ........................................................... 138 


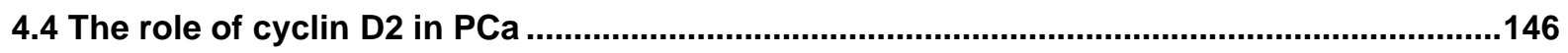

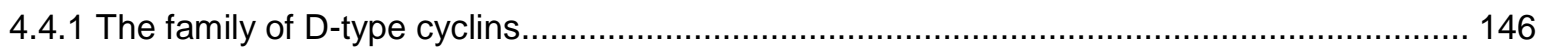

4.4.2 Does cyclin D2 act as an oncogene or a tumor suppressor gene in $\mathrm{PCa}$ ? ......................... 147

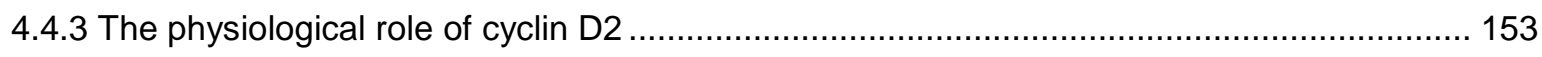

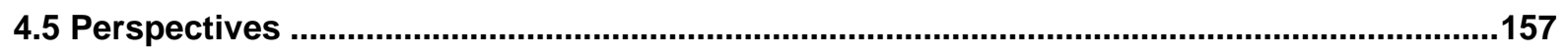

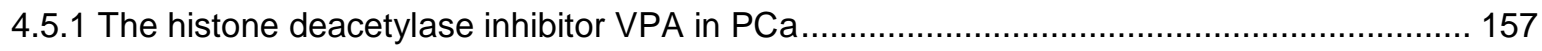

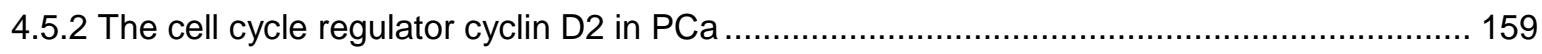

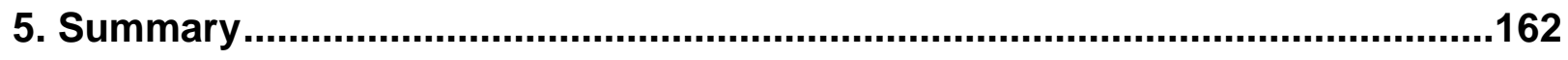

5.1 The histone deacetylase inhibitor valproic acid in $\mathrm{PCa}$...................................................162

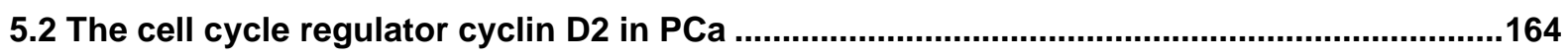

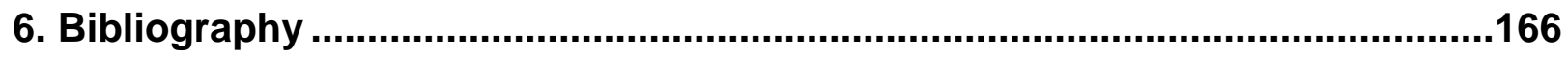

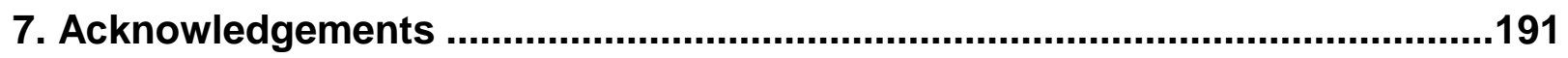




\section{List of Abbreviations}

A

$A b$

ad.

Amp

Ang1

app.

ATP

$\mathrm{Bp}$

BSA

C

${ }^{\circ} \mathrm{C}$

CAM

CCND

Cdk

cDNA

Cp

$\mathrm{Ct}$

CTP

Cxcl15

DAPI

$\mathrm{ddH}_{2} \mathrm{O}$

DHT

DMEM

DMSO

DNA

dNTP

DPBS

DTT

E.coli

EDTA

e.g.

EGFP

et al.
Nucleobase adenine

Antibody

add...to...

Ampicillin

Angiopoietin 1

approximately

Adenosine triphosphate

Base pair

Bovine serum albumin

Nucleobase cytosine

Degree Celsius

(Chicken) Chorioallantoic membrane

Cyclin

Cyclin-dependent kinase

Complementary DNA

Ceruloplasmin

Threshold cycle

Cytosine triphosphat

Chemokine (C-X-C motif) ligand 15

4',6-diamidino-2-phenylindole

Bi-destilled water

Dihydrotestosterone

Dulbecco's Modified Eagle Medium

Dimethyl sulfoxide

Deoxyribonucleic acid

Deoxynucleoside triphosphate

Dulbecco's phosphate-buffered saline solution

Dithiothreitol

Escherichia coli

Ethylenediaminetetraacetic acid

exempli gratia, for example

Enhanced green fluorescent protein

et alteri 
Fig

FKS

Flt-1

FW

G

$\mathrm{G}$

GAPDH

$\mathrm{H}$

Hif1a

HPRT

HRP

i.e.

Kan

$\mathrm{Kb}$

$K d r$

$\mathrm{kDa}$

Kras

LDS

Lif

Luc

M

$\mathrm{M}$

$\mathrm{mA}$

$\mathrm{Mg}$

$\mathrm{MgCl}_{2}$

Min

MI

$\mathrm{mM}$

mod.

mRNA

MTS

$\mathrm{N}$

$\mathrm{NaCl}$
Figure

Fetal calf serum

Vascular endothelial growth factor receptor 1

forward

Nucleobase guanine

Gram

Glyceraldehyde 3-phosphate dehydrogenase

Hour

Hypoxia inducible factor 1, alpha subunit

Hypoxanthine-guanine phosphoribosyltransferase

Horse radish peroxidase

id est

Kanamycin

Kilo base pair

Kinase insert domain receptor, Vascular endothelial growth

factor receptor 2

Kilo Dalton

Kirsten rat sarcoma viral oncogene homolog

Lithium dodecyl sulfate

Leukemia inhibitory factor

Luciferase

Milli $=10^{-3}$, murin

Molar

Milliampere

Milligram

Magnesium chloride

minutes

Milliliter

Millimolar

Modified

Messenger RNA

3-(4,5-dimethylthiazol-2-yl)-5-(3carboxymethoxyphenyl)-2-

(4-sulfophenyl)-2H-tetrazolium

Nano $=10^{-9}$

Sodium chloride 


\begin{tabular}{|c|c|}
\hline $\mathrm{Nm}$ & Nanometer \\
\hline OD & Optical density \\
\hline Oligo(dT) & 15-25 Desoxythymidine \\
\hline $\mathrm{o} / \mathrm{n}$ & Overnight \\
\hline$P$ & Pico $=10^{-12}$ \\
\hline PAGE & Polyacrylamide gel electrophoresis \\
\hline$P B G D$ & Porphobilinogen deaminase \\
\hline PBS & Phosphate buffered saline solution \\
\hline $\mathrm{PCa}$ & Prostate carcinoma \\
\hline PCR & Polymerase chain reaction \\
\hline Pecam-1 & Platelet endothelial cell adhesion molecule-1 \\
\hline \multirow[t]{2}{*}{$\mathrm{pH}$} & negative decimal logarithm of the hydrogen ion \\
\hline & concentration \\
\hline PIN & Prostatic intraepithelial neoplasia \\
\hline PMS & Phenazine methosulfate \\
\hline $\mathrm{P} / \mathrm{S}$ & Penicillin/Streptomycin \\
\hline$P S A$ & Prostate-specific antigen \\
\hline Pten & Phosphatase and tensin homologue \\
\hline Ptprn & Protein tyrosine phosphatase, receptor type, $N$ \\
\hline PVDF & Polyvinylidene fluoride \\
\hline qRT-PCR & Quantitative real-time PCR \\
\hline$R b$ & Retinoblastoma \\
\hline \multirow[t]{2}{*}{ Rcbtb2 } & Regulator of chromosome condensation (RCC1) and BTB \\
\hline & (POZ) domain containing protein 2 \\
\hline $\operatorname{Rev}$ & Reverse \\
\hline RKI & Robert Koch-Institute \\
\hline RNA & Ribonucleic acid \\
\hline RNase & Ribonuclease \\
\hline rpm & rounds per minute \\
\hline $\mathrm{RT}$ & room temperature \\
\hline RT-PCR & reverse Transcriptase PCR \\
\hline SDS & Sodium dodecyl sulfate \\
\hline sec & Seconds \\
\hline shRNA & short hairpin RNA \\
\hline siRNA & small interfering RNA \\
\hline
\end{tabular}


SV

sVegfr2

$\mathrm{T}$

Tab.

Taq.

TBP

TBS(-T)

Tie-1

TRAMP

Uchl1

UTP

UV

$\mathrm{V}$

Vegf

Vegfa

Vegfb

Vegfb

Vegfr1 (Flt1)

Vegfr2 (Kdr)

Vol.

vs.

VPA

$\mathrm{Wb}$

w/o

$\mathrm{w} / \mathrm{v}$

$x \mathrm{G}$

$X-G a l$

$\mu$

$\mu \mathrm{g}$

$\mu l$

$\mu \mathrm{m}$

$\mu \mathrm{M}$
Simian virus

Soluble vascular endothelial growth factor receptor 2

Nucleobase Thymine, temperature

Table

Thermus aquaticus

TATA-binding protein

Tris-buffered saline solution (with Tween)

Tyrosine kinase with immunoglobulin-like and EGF-like domains 1

Transgenic adenocarcinoma of mouse prostate

Ubiquitin carboxyl-terminal esterase $L 1$

Uridine-5'-triphosphate

Ultraviolet

Volt

Vascular endothelial growth factor

Vascular endothelial growth factor $A$

Vascular endothelial growth factor $B$

Vascular endothelial growth factor $C$

Vascular endothelial growth factor receptor 1

Vascular endothelial growth factor receptor 2

Volume

Versus

Valproic acid

Western blot

Without

Mass concentration

Multiple of acceleration of gravity

5-bromo-4-chloro-3-indolyl- $\beta$-D-galactopyranoside

Micro $=10^{-6}$

Microgram

Microliter

Micrometer

Micromolar 
$-1-$

\section{Introduction}

\subsection{The prostate carcinoma- incidence and risk factors}

Worldwide, prostate cancer (PCa) is with an estimated 1.1 million new cases the second most frequently diagnosed cancer in men and the fifth leading cause of cancer-related death in men with an estimated 307.500 deaths in 2012. Thereby, the majority of new cases (about two-thirds) was diagnosed in economically developed countries (American Cancer Society, Global cancer facts and figures, $3^{\text {rd }}$ edition, 2015). In Germany, PCa is with 63.710 (25.7\% of total cancer diagnosis, Fig. 1.1) newly diagnosed cases in 2011 still the most frequent diagnosed cancer in men, although the frequency decreased slightly from 67.300 newly diagnosed cases in the year 2010. In the list of cancer-related deaths in men in Germany PCa is listed with 12.957 deaths (10.5\%, Fig. 1.1) at the third place, preceded only by lung cancer (24.8\%) and intestinal cancer (11.5\%; Robert Koch-Institute (RKI) and German Centre for Cancer Registry Data (ZfKD), Krebs in Deutschland 2011/2012). Worldwide, the number of newly diagnosed PCa cases will even further increase due to recording of prostate-specific antigen (PSA) testing in countries where it was not yet commonly used for PCa diagnosis. In contrast, PCa death rates will decrease due to improved treatment and/or early detection. To date, the 5-year relative survival rate for PCa patients in the USA is $97 \%$, whereas in Germany it is $91 \%$ (American Cancer Society, Global cancer facts and figures, $3^{\text {rd }}$ edition, 2015).

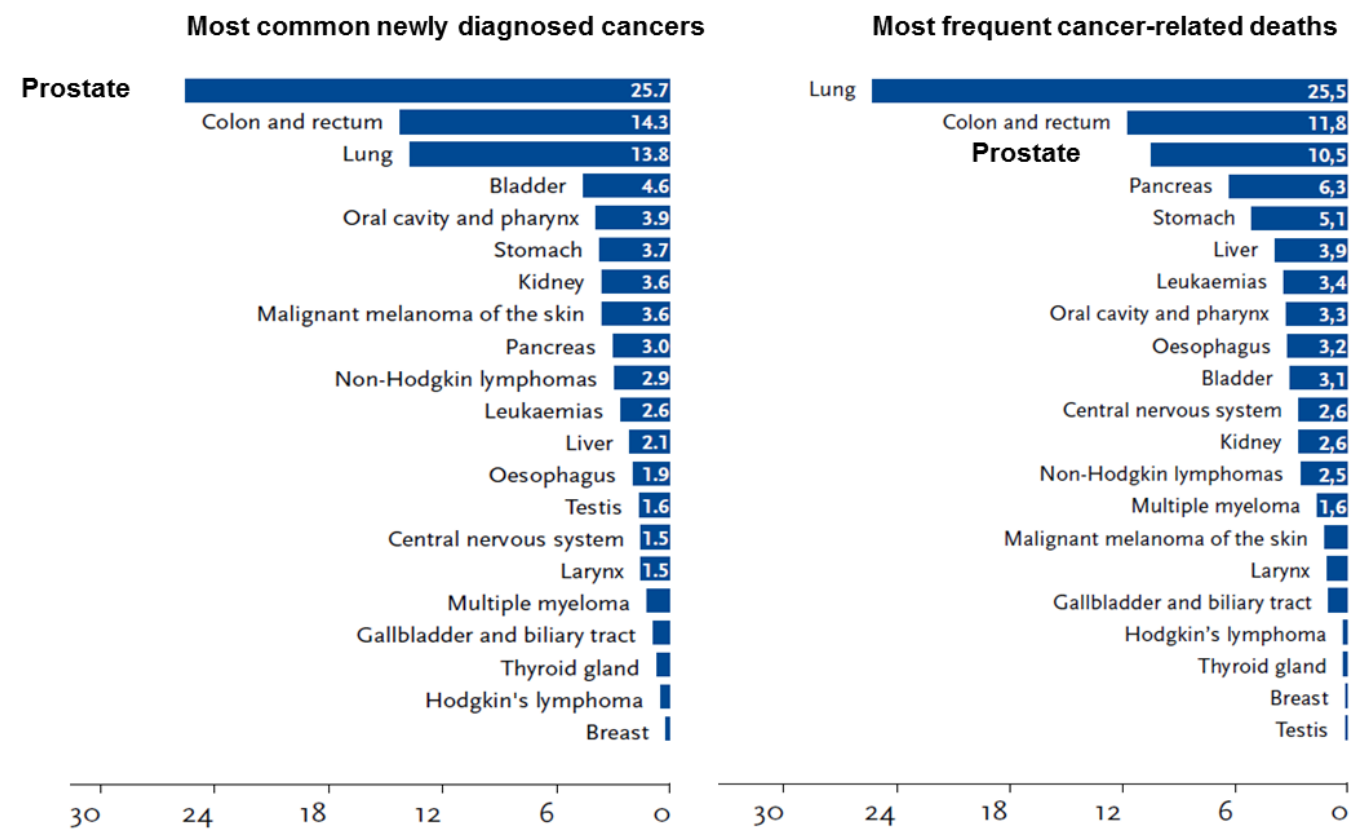

Fig.1.1: Most frequent tumor sites and most frequent cancer-related deaths as a percentage of all cancer cases in Germany, 2011. PCa is the most common newly diagnosed cancer in men in Germany with $25.7 \%$ of all 
new cancer cases. It is the third leading cause of cancer-related death with $10.5 \%$ of all cancer related deaths. Modified from: Robert Koch-Institute and German Centre for Cancer Registry Data (ZfKD), Krebs in Deutschland 2011/2012.

Several risk factors for the development of $\mathrm{PCa}$ are known and depicted in Fig. 1.2. The main risk factor for the development of PCa is increasing age. A 35-year old male in Germany has a $0.1 \%$ risk to develop PCa in the next 10 years, whereas a 75 -year old male has a $6 \%$ risk to develop PCa within 10 years (RKI and German Centre for Cancer Registry Data (ZfKD), Krebs in Deutschland 2011/2012). Other risk factors include the level of the male sex hormone testosterone, African ancestry and a family history of the disease, although the underlying molecular mechanisms remain unclear. In Asia, where the incidence rates for PCa are lowest (2012: $10.5 \%$ and $4.5 \%$ in East and South-Central Asia, respectively) the mortality rates are rising. Presumably this is due to an increased economic development and western lifestyle with increased consumption of animal fat, obesity and physical inactivity (American Cancer Society, Global cancer facts and figures, $3^{\text {rd }}$ edition, 2015), indicating that diet and life style might also influence the risk to develop PCa.

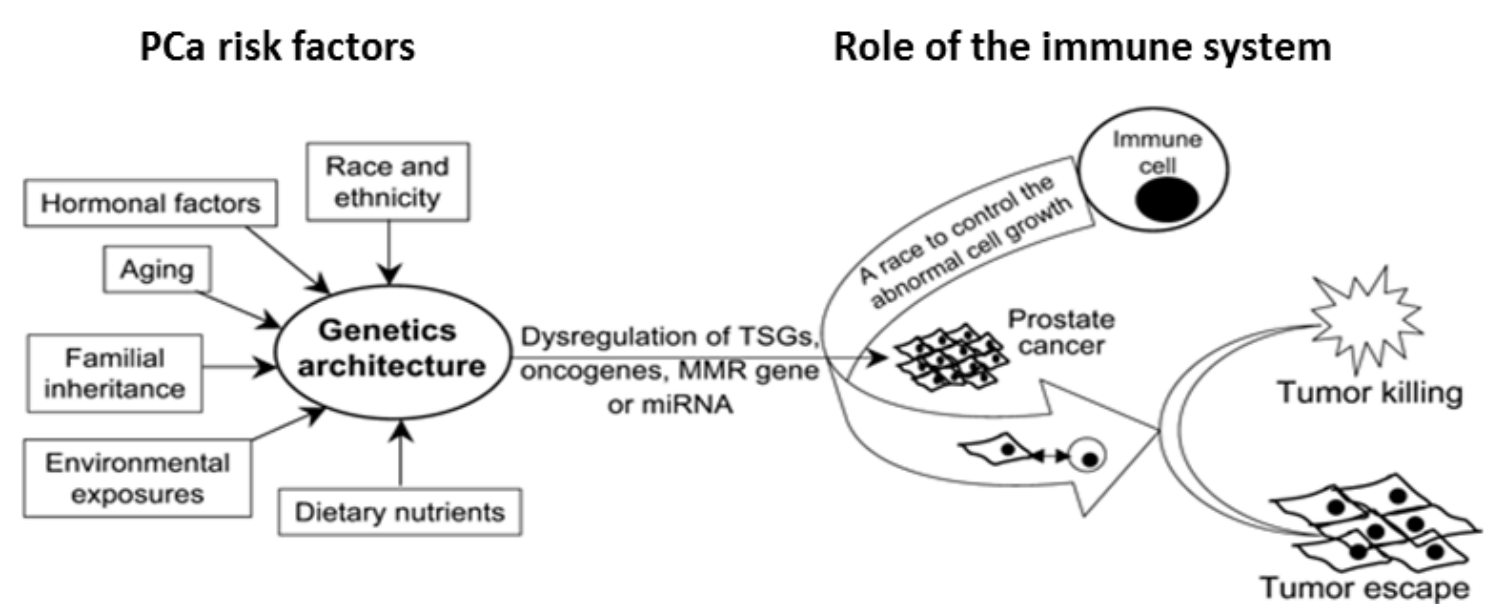

Fig.1.2: Risk factors involved in initiation and development of PCa and the role of the immune system. Risk factors collectively influence genetic and epigenetic factors, leading to dysregulation of tumor suppressor genes (TSGs), oncogenes, mismatch repair genes (MMR) and micro RNAs (miRNA). The balanced cell growth is shifted toward excessive cell growth leading to development of PCa. Immune cells, which are primarily responsible for the killing of tumor cells, are unable to take care of the great amount of cancer cells, resulting in tumor cells escape and further growth. Modified from Karan et al. 2008.

\subsection{Diagnosis and standard therapy options for PCa}

Since the late 1980s PSA testing is being used as a tumor marker for malignancy of the prostate. Today, it represents the most important tool to detect PCa in very early stages, even before the 
patient notices any symptoms. But its' use is still controversially discussed, mainly due to frequent overdiagnosis (tumors which would never have become clinically evident; estimated $23 \%-42 \%$ for PSA screen-detected cancers, Draisma et al. 2009) and due to the large potential of serious side effects of treatment options once PCa has been diagnosed. Therefore, in Germany health insurance is not covering the PSA screening. But it is still offered as individual health service by many medical practices and the patient has to pay for it himself. Yearly routine early detection screening for PCa in Germany starts for men at the age of 45 years and includes questioning of health problems like experience with weak or interrupted urine flow, the inability to urinate or difficulty starting or stopping the urine flow, the need to urinate frequently, especially at night, blood in the urine and pain or burning with urination. Also the prostate gland and the lymph nodes will be examined by palpation (RKI and German Centre for Cancer Registry Data (ZfKD), Krebs in Deutschland 2011/2012). Once the PSA test reveals a conspicuous value the only way to find out whether the patient really has a PCa is by examination of prostate biopsies. An elevated PSA value can not only be caused by PCa, but also by benign prostatic hyperplasia (BPH).

The treatment options for PCa depend on the patients' age as well as on the stage of the cancer (Fig. 1.3). According to the International Union against Cancer (UICC) PCa can be described by four mayor stages, stage one (T1) being a tumor that cannot be felt and is not visible on imaging. Stage two (T2) describes a tumor that is confined to the prostate whereas stage three (T3) describes a tumor that has spread beyond the prostatic capsule. Stage four (T4) describes a tumor that has metastasized at distant organs.

Men with a less aggressive tumor and older men will not be treated immediately since the treatment options cause severe side effects. Instead, they will be carefully observed (active surveillance/watchful waiting) and only when the tumor becomes more aggressive the treatment will be initiated. Early stage PCa (organ specific, no metastatic spread) that is not subject to careful observation can be treated by surgery (radical prostatectomy: open, laparoscopic or robotic-assisted), external beam radiation or radioactive seed implants (brachytherapy), which reduces radiation exposure of surrounding tissue compared to external beam radiation (American Cancer Society, Global cancer facts and figures, $3^{\text {rd }}$ edition, 2015). But all three early stage treatment options are associated with severe side effects, mainly effecting urination and erection. For advanced early stage disease the hormone depletion therapy, which cause withdrawal of androgens (testosterone and dihydrotestosterone), is used alongside to surgery or radiation therapy. This therapy approach, which can be either chemically or surgically, is not curative anymore, it solely prevents PCa cells from growing. Thereby, it slows tumor progression down for 2-4 years before recurrence, marking the progression to metastatic castration-resistant 
PCa (mCRPC) because PCa cells gradually developed resistance to hormonal therapy. Advanced stages of $\mathrm{PCa}$, indicated by metastatic spread or castration resistance, are also treated by hormone depletion therapy since the tumor shrinkage and/or limited expansion can help relieve pain and other symptoms. Other treatment options include radiation therapy in combination with hormone depletion therapy and chemotherapy with docetaxel. Docetaxel was the first known agent to extend survival in men with mCRPC, a cancer that has come back or has progressed while being treated with hormonal therapy and is invariably fatal (Harris et al. 2009).

During the last six years several new treatments have been tested in randomized phase III trials and approved for the treatment of mCRPC since they have been shown to improve survival (Fig. 1.3, bottom). The new treatment options include the immunotherapy sipuleucel-T (removed from the German market for unknown reasons in June 2015, Kantoff et al. 2010), cabazitaxel, which is a novel tubulin-binding taxane drug with antitumor activity in docetaxel-resistant cancers (de Bono et al. 2010), abiraterone acetate, which is an inhibitor of androgen biosynthesis (de Bono et al. 2011), radium-223, which delivers cytotoxic radiation to the sites of bone metastases (ElAmm and Aragon-Ching 2015) and enzalutamide, which targets multiple steps in the androgenreceptor-(AR) signaling pathway, the major driver of PCa growth (Scher et al. 2012).

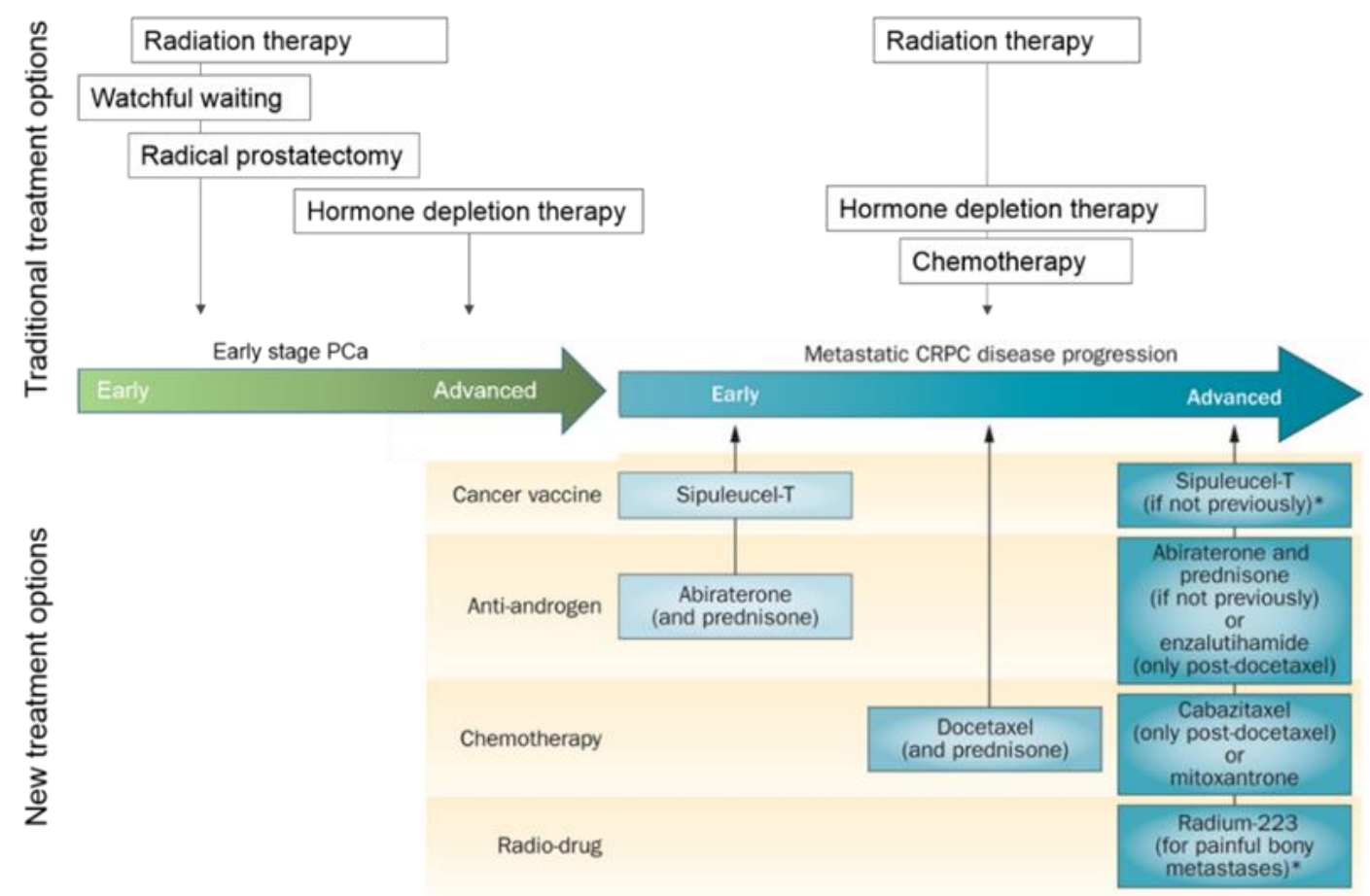

Fig.1.3: Treatment options for patients with PCa. At the top, the traditional treatment options for early stage PCa and $\mathrm{mCRPC}$ are indicated. At the bottom, the newly approved treatment options for $\mathrm{MCRPC}$ are depicted, which were developed during the last six years. Although, sipuleucel-T was removed from the German market in June 2015 for unknown reasons. Modified from Jha et al. 2014. 


\subsection{Therapeutic perspectives in PCa}

Current research is investigating the potential of several alternative treatment options in $\mathrm{PCa}$ prevention and therapy. A list of different treatment approaches under current investigation, including hormone therapy, chemotherapy and epigenetic modulators, together with examples of tested drugs and their targets are demonstrated in Table 1.1. The hormone therapy, especially the androgen deprivation therapy, is of great interest for the treatment of PCa treatment since androgens, such as testosterone and dihydrotestosterone (DHT), stimulate the growth of $\mathrm{PCa}$ cells. Established androgen receptor (AR) pathway-targeting agents display clinical efficacy in mCRPC, but dose-limiting side effects remain problematic for all current agents. One novel, promising agent under current investigation is ARN-509, a synthetic biaryl thiohydantoin compound and competitive AR inhibitor. It was shown to exhibit characteristics predicting a higher therapeutic index with a greater potential to reach maximally efficacious doses in man than current AR antagonists (Clegg et al. 2012). Another phase I study including thirty patients with progressive CRPC that received daily oral ARN-509, showed that this drug is safe and well tolerated, displayed dose-proportional pharmacokinetics and demonstrated anti-tumor activity (Rathkopf et al. 2013). Another promising strategy for PCa control is chemoprevention which is the use of naturally (e.g. dietary) occurring or synthetic agents as a way to prevent, delay, or slow the process of carcinogenesis. A clinical trial in which PCa patients were treated orally with the hormone vitamin D3 revealed an increase in prostate calcitriol levels and a modestly lowered PSA level (Wagner et al. 2013a). Calcitriol was shown to exert several anti-inflammatory actions in prostate cells which contribute to its potential as a chemopreventive and therapeutic agent in PCa (Krishnan et al. 2007).

Epigenetic modulators, such as microRNA (miRNA)-based therapeutic strategies are also under intensive investigation. miRNAs modulate the activity of key cell signaling networks by regulating the translation of pathway component proteins. Therefore, pharmacological targeting of miRNAs that regulate cancer cell signaling networks, either by promoting (using miRNA-supplementation) or by suppressing (using antisense oligonucleotide-based strategies) miRNA activity is a promising opportunity for therapeutic intervention in cancer in general (Sharma and Ruppert 2015). In PCa, miRNAs are rather implied in miRNA profiling which can be a useful diagnostic and prognostic tool to assist in the recognition of PCa with aggressive behavior (Schaefer et al. 2010, Walter et al. 2013). Although, studies using miRNA-34a in PCa showed that it has a proapoptotic effect and can suppress malignancy in human PCa cells by modulating c-Myc transcriptional complexes (Yamamura et al. 2012, Li et al. 2014). 
Phytochemicals such as sulforaphane (SFN) and 3,3'-diindolylmethane (DIM) are promising chemopreventive agents in PCa (Hsu et al. 2011, Wong et al. 2014). SFN and DIM, derived from glucosinolates in cruciferous vegetables like cabbage and broccoli, are epigenetic modulators which have been shown to alter promoter methylation in distinct sets of genes in PCa cells. SNF has been shown to de-methylate the cyclin D2 promoter in PCa cells, resulting in cyclin D2 reexpression (Hsu et al. 2011). Hyper-methylation of the cyclin D2 promoter, a major regulator of the cell cycle, is correlated with PCa progression, and restoration of cyclin D2 expression exerts anti-proliferative effects in LNCaP PCa cells (Kobayashi et al. 2009).

Regulation of gene expression as a therapeutic approach for PCa is also exerted by the histone deacetylase inhibitor valproic acid (VPA). Its therapeutic benefits have been studied for many tumor entities in vitro and in vivo, including cancer of the bladder (Ozawa et al. 2010, Vallo et al. 2011, Byler et al. 2012), hepatocellular cancer (Machado et al. 2011), head and neck cancer (Gan et al. 2012), pancreatic cancer and colon cancer (Jones et al. 2008, Venkataramani et al. 2010), renal cell carcinoma (Jones et al. 2009), small cell lung cancer (Hubaux et al. 2010), cervical cancer (Sami et al. 2008) and breast cancer (Fortunati et al. 2008) as well as in clinical trials for breast cancer, lung cancer, pancreatic cancer and ovarian cancer (Arce et al. 2006, Candelaria et al. 2007, Munster et al. 2009, Chateauvieux et al. 2010).

Similar to SNF, VPA was shown to specifically re-express cyclin D2 in mouse PCa cells in a time- and concentration-dependent manner (Witt et al. 2013). Several VPA in vitro and in vivo studies in PCa resulted in inhibition of proliferation (Xia et al. 2006, Annicotte et al. 2006, Shabbeer et al. 2007, Gao et al. 2007, Chou et al. 2011). Thereby, proliferation inhibition was due to functional effects of VPA treatment inducing either growth arrest, cell death, senescence or anti-angiogenic properties (Shabbeer et al. 2007, Gao et al. 2007, Wedel et al. 2011). Nonetheless, the underlying molecular mechanisms for the VPA-induced inhibition of PCa cell proliferation remain unsolved. Witt et al. (2013) could also show that treatment of murine PCa cells $2 \mathrm{E}$ with VPA resulted not only in proliferation inhibition but also in migration and invasion inhibition. These functional effects were concentration- and time-dependent. Microarray analysis of $2 \mathrm{E}$ cells treated for 24 hours with VPA identified several candidate genes that were deregulated after VPA treatment, including the angiogenesis-related genes ceruloplasmin 1 and 2 (Cp1, Cp2) and chemokine (C-X-C motif) ligand 15 (Cxc/15) among others (Witt et al. 2013). After VPA treatment their expression was decreased in a concentration- and time-dependent manner. 
$-7-$

Table 1.1: Examples of therapy options under investigation for PCa treatment. PCa therapy can be approached in several ways, including hormone therapy, chemotherapy and epigenetic modulations. Here, therapy options under current investigation, along with their effect/target, example drugs and the corresponding studies are depicted.

\begin{tabular}{|c|c|c|c|}
\hline Therapy option & Effect/Target & Drug & Studies \\
\hline $\begin{array}{l}\text { Hormone } \\
\text { therapies }\end{array}$ & $\begin{array}{ll}- & \text { lowered PSA level } \\
\text { and anti- } \\
\text { inflammatory } \\
\text { actions } \\
\text { - } \begin{array}{l}\text { androgen receptor } \\
\text { (AR) antagonist }\end{array}\end{array}$ & 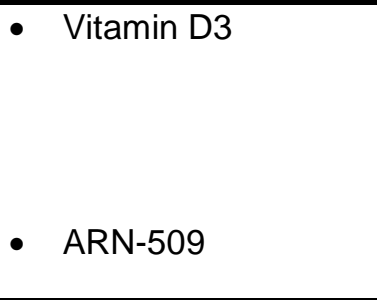 & $\begin{array}{l}\text { - Krishnan et al. 2007, } \\
\text { Wagner et al. 2013b } \\
\text { - Clegg et al. 2012, } \\
\text { Rathkopf et al. } 2013\end{array}$ \\
\hline Chemotherapies & $\begin{array}{l}\text { extended survival } \\
\text { and inhibition of } \\
\text { other tumorigenic } \\
\text { effects }\end{array}$ & $\begin{array}{l}\text { docetaxel in } \\
\text { combination with } \\
\text { anti-angiogenic } \\
\text { agents, immune } \\
\text { modulators or } \\
\text { miscellaneous } \\
\text { agents } \\
\end{array}$ & 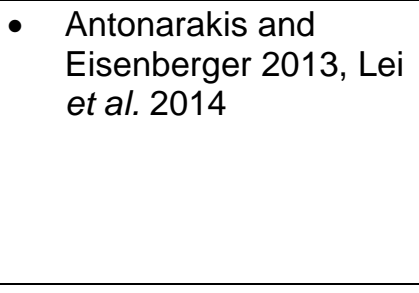 \\
\hline $\begin{array}{l}\text { Epigenetic } \\
\text { modulators }\end{array}$ & $\begin{array}{l}\text { - gene silencing } \\
\text { - } \begin{array}{l}\text { altered promoter } \\
\text { methylation }\end{array} \\
\text { - histone } \\
\text { deacetylase } \\
\text { inhibitor }\end{array}$ & 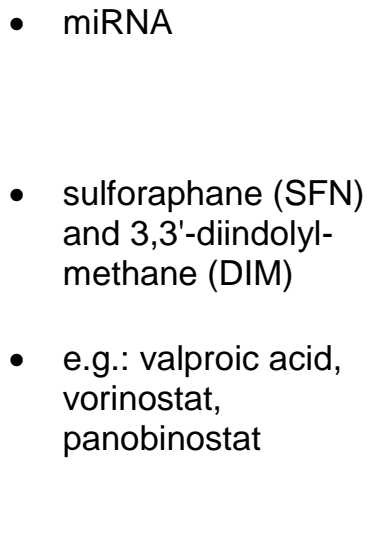 & $\begin{array}{l}\text { - Yamamura et al. 2012, } \\
\text { Li et al. 2014, Sharma } \\
\text { and Ruppert } 2015 \\
\text { - Hsu et al. 2011, Wong } \\
\text { et al. } 2014 \\
\\
\text { - Sharma et al. 2008, } \\
\text { Braiteh et al. 2008, } \\
\text { Munster et al. 2009, } \\
\text { Kaushik et al. 2015 }\end{array}$ \\
\hline
\end{tabular}

The anti-angiogenic effect of in vitro or in vivo VPA treatment has already been described in a few studies for PCa (Shabbeer et al. 2007, Gao et al. 2007, Sidana et al. 2012). Sidana et al. (2012) showed that DU145 and LNCaP xenografts showed a statistically significant decrease in microvessel density (MVD) following VPA treatment. MVD is a quantitative indication of the risk of tumor prognosis and metastases risk in various solid malignant tumors and it was evaluated by CD34 expression in the xenografts. CD34 is commonly used as a marker for tumor neovascularization since it is expressed by endothelial cells of blood vessels (Fina et al. 1990). Gao et al. (2007) could show that xenografts derived from the human PCa cell line PC-3 that were treated with VPA had a reduced expression of vascular endothelial growth factor (VEGF), the main angiogenic stimulator in normal and metastatic tissue (Ferrara 1995). For other cells and cancer types the anti-angiogenic effects of in vitro and in vivo VPA treatment have also been described (Michaelis et al. 2004), including gliomas (Osuka et al. 2012) and acute myeloid leukemia (Zhang et al., 2014). 
Anti-angiogenic treatment is an emerging and promising approach for the treatment of CRPC (Kluetz et al. 2010, Mukherji et al. 2013). By inhibiting tumor angiogenesis the metastatic spread as well as the growth of the primary tumor itself will be inhibited. Angiogenesis seems to play an important role in the pathophysiology of PCa (Weidner et al. 1993) and many anti-angiogenic agents have proven to be effective in the treatment of several solid tumors, including renal-cell carcinoma (Motzer et al. 2007), colorectal cancer (Hurwitz et al. 2004) and non-small cell lung cancer (Sandler et al. 2006). Bevacizumab, a monoclonal antibody against VEGF, in combination with other chemotherapy drugs proved to be very effective in many different cancers, but not so in PCa. A large phase III study in patients with CRPC using bevacizumab in combination with docetaxel chemotherapy did not improve overall survival (OS) of these patients and was even associated with greater toxicity (Kelly et al. 2012). Docetaxel in combination with other anti-angiogenic agents also did not increase the OS or progression-free survival (PFS), as shown by several clinical trials with CRPC patients. In contrast, it might even increase the risk for treatment-related mortality (Lei et al. 2014). Clinical phase III trials investigating the benefits of the anti-angiogenic agents sunitinib, a receptor tyrosine kinase inhibitor, or lenalidomide, an immune-modulating agent, in CRPC patients were even discontinued due to futility (Mukherji et al. 2013). Nonetheless, the rationale for the use of anti-angiogenic therapy in patients suffering from CRPC remains strong since PCa progression is strongly dependent on angiogenesis. It was shown that angiogenesis measured as microvessel density (MVD) is associated with tumor stage as well as WHO grade and is an independent predictor of clinical outcome. Besides, VEGF expression correlates positively with tumor stage and outcome (Strohmeyer et al. 2000). Therefore, novel anti-angiogenic agents are being under constant investigation. One promising approach is targeting the dual VEGFR2/MET with the tyrosine kinase inhibitor cabozantinib, which showed improvements in bone scans and pain response (Lee and Smith 2013, Smith et al. 2013). Generally, the role of anti-angiogenic treatment in PCa has yet to be defined.

The reduced Cp1, Cp2 and Cxc/15 expression after VPA treatment observed by Witt et al. (2013), together with the VPA-induced anti-angiogenic effects observed by Gao et al. (2007), Shabbeer et al. (2007) and Sidana et al. (2012) highlight the beneficial effects of VPA treatment in PCa by acting as an anti-angiogenic factor and thereby preventing the tumor from growing and spreading. To further investigate the molecular mechanisms underlying the VPA-mediated anti-angiogenic properties in PCa was subject of this project. 


\subsection{Cyclin D2 in general}

As mentioned above, for several PCa therapies, including treatment with the HDI VPA or with sulforaphane, it was shown that cyclin D2 will be specifically re-expressed, which usually is downregulated due to hypermethylation of the promoter, and might contribute to the anticancerous effects observed.

Cyclin D2 belongs to the highly conserved family of D-type cyclins, together with cyclin D1 and cyclin D3. Although the literature often refers to cyclin D in general, there are significant differences in the structure of the three D-type cyclins (Fig. 1.4) as wells as in their function.

The D-type cyclins consist of different domains for which the percentage in homology to the cyclin D1 sequence is indicated in Fig. 1.4. The greatest homology occurs in the cyclin box which mediates the interaction with cyclin-dependent kinase 4 (CDK4) and CDK6 and is also needed for the interaction with cyclin-dependent kinase inhibitors. The interaction with the retinoblastoma $(\mathrm{Rb})$ protein is mediated by the LXCXE motif, which is also conserved in all three D-type cyclins. Cyclin D1 contains as sole exception a LLXXXL motif which facilitates binding to specific transcription factors. Another common feature of all three D-type cyclins is a threonine residue near the $\mathrm{C}$ terminus which triggers ubiquitin-mediated degradation when phosphorylated. It is located within the PEST domain that is characteristic of proteins that are rapidly turned over (Musgrove et al. 2011).

\begin{tabular}{|c|c|c|c|c|c|}
\hline D1 & He a a a a & & & & 295 aа \\
\hline \multicolumn{4}{|c|}{ K112 } & \multicolumn{2}{|c|}{ T286 } \\
\hline D2 & $54 \%$ & $78 \%$ & $56 \%$ & $48 \%$ & 290 aа \\
\hline \multicolumn{4}{|c|}{ K111 } & \multicolumn{2}{|c|}{ T280 } \\
\hline D3 & $49 \%$ & $73 \%$ & $46 \%$ & $36 \%$ & 292 аa \\
\hline \multicolumn{4}{|c|}{ K112 } & \multicolumn{2}{|c|}{$\mathrm{T} 283$} \\
\hline \multicolumn{3}{|r|}{ Cyclin box } & & \multirow{2}{*}{\multicolumn{2}{|c|}{ PEST }} \\
\hline & LXCXE r & $\square$ LLXXXL motif & & & \\
\hline
\end{tabular}

Fig. 1.4: Schematic presentation of the protein domain structure of the three D-type cyclins. The three D-type cyclins consist of different domains of which the percentage in homology to the sequence of cyclin D1 is indicated. Cyclin D1, cyclin D2 and cyclin D3 share a LXCXE motif which mediates interaction with the retinoblastoma (Rb) protein, a cyclin box which mediates the interaction with cyclin-dependent kinases (CDK4 and CDK6) and cyclindependent kinase inhibitors and has the greatest homology between the D-type cyclins, a C-terminal PEST domain which is characteristic of proteins that are turned over rapidly, and a C-terminal threonine residue which triggers ubiquitin-mediated degradation once phosphorylated. Cyclin D1 comprises as sole exception a LLXXXL motif which facilitates binding to specific transcription factors. Modified from Musgrove et al. 2011. 
The D-type cyclins exhibit their major function in regulating the cell cycle. The cell cycle is divided into the G1, S, G2 and M phase plus the G0 rest phase. Transition of the cell from one phase to the next is strictly regulated by cyclins which serve as regulatory co-factors for cyclin-dependent kinases (CDKs) which are inactive in the absence of a partner cyclin. The specific complexes that result from binding of the cyclin to its' particular CDK activate the catalytic subunit of the CDK resulting in phosphorylation of target genes (Matsushime et al. 1992). This leads to either activation or inactivation of target proteins which ultimately leads to the progression to the next phase of the cell cycle. Different combinations of cyclin-CDK complexes, as well as the expression of CDK-inhibitors operate during different phases of the cell cycle and determine the target genes. CDKs are expressed continuously whereas the cyclins are expressed at specific stages of the cell cycle (Voorzanger-Rousselot et al. 2007). For example, cyclin D2 binds specifically to CDK4 and CDK6. The resulting complex causes phosphorylation of the tumor suppressor gene retinoblastoma $(\mathrm{Rb})$ growth-inhibitory complex. Once phosphorylated, this complex releases the E2F transcription factor which controls various genes required for DNA synthesis and cell cycle control and thus $\mathrm{Rb}$ is no longer able to prevent the $\mathrm{G} 1$ arrest and the cell progresses to the $S$ phase (Weinberg 1995). In tumor cells many of the regulatory mechanism of the cell cycle can be deregulated. According to Musgrove et al. (2011), especially the abnormal expression of the D-type cyclins and their CDKs is linked to cancer development and progression.

\subsection{Cyclin D2 in PCa}

In different tumor entities different expression patterns of cyclin D2 are observed. On the one hand, in ovarian epithelial carcinomas ( $23 \%$ of 81 patients, Milde-Langosch and Riethdorf 2003), colon cancer (53\% of 57 patients, Mermelshtein et al. 2005) and gastric cancer (34.2\% of 260 patients, Takano et al. 2000) cyclin D2 is frequently overexpressed. On the other hand, in breast cancer (44\% of 109 patients, Evron et al. 2001, Fischer et al. 2002), lung cancer (57\% of 56 patients with small cell lung cancer (SCLC), Virmani et al. 2003), pancreatic cancer (65.1\% of 109 patients, Matsubayashi et al. 2003) and some gastrointestinal tumors (48.9\% of 23 patients, Yu et al. 2003) cyclin D2 expression is frequently downregulated. The downregulation of cyclin D2 in these cancers is mainly due to a hypermethylation of the cyclin D2 promoter region. In PCa patients it was shown that, depending on the stage of the tumor, the cyclin D2 promoter was increasingly hypermethylated resulting in epigenetic silencing (32\% of $101 \mathrm{PCa}$ samples, Padar et al. 2003). Henrique et al. could show a significant inverse correlation between the cyclin D2 methylation status and the expression levels in prostatic tissue (Henrique et al. 2006). 
Methylation of the cyclin D2 promoter in PCa correlates thereby with clinico-pathological features of faster tumor progression and poor prognosis (Padar et al. 2003, Rosenbaum et al. 2005). Witt et al. could show by immunohistochemistry of PCa sections that indeed only the PCa cell lack cyclin D2 expression and the cells of the intercellular space as well as of the surrounding healthy tissue still exhibit cyclin D2 expression (Witt et al. 2013). In contrast, cyclin D1 expression was increased in PCa tissue. This was also shown by Drobnjak et al. (2000). They could demonstrate that overexpression of cyclin D1 is associated with metastatic spread of PCa to the bone (Drobnjak et al. 2000) highlighting the function of cyclin D1 as an oncogene in PCa (Ewen and Lamb 2004). The role of cyclin D2 in PCa further remains unclear, but studies conducted by Witt et al. (2013) suggest that it could act as a tumor suppressor gene in PCa. Witt and coworkers could show that the mouse PCa cell line 2E as well as the human PCa cells DU145, LNCaP and PC-3 which displayed a very low, not detectable basal cyclin D2 expression due to hypermethylation of the promoter region, specifically re-expressed cyclin D2 (in a time- and concentration-dependent manner) when treated with VPA or other HDIs. Treatment of the PCa cells with the HDI VPA led to increased acetylation of the cyclin D2 promoter and thereby resulting in its activation (Witt et al. 2013). Simultaneously, the proliferation rate of VPA-treated PCa cells was significantly reduced. In contrast, fibroblast cells, such as NIH/3T3 cells among four other tested cell lines, exhibited a high basal cyclin D2 expression which was not further increased after VPA treatment. An inhibition of the proliferation rate after VPA treatment could solely be observed in one out of five fibroblast cell lines (L-cells, Witt et al. 2013). The hypothesis that cyclin D2 could act as a tumor suppressor in PCa is further supported by a publication by Kobayashi et al. (2009) in which it is stated that restoration of the cyclin D2 expression in the human PCa cells LNCaP inhibited cell proliferation (Kobayashi et al. 2009). Moreover, in nonsmall cell lung cancer, a reduced cyclin D2 expression is correlated with a poor recurrence-free survival (Ko et al. 2012). The findings by Witt et al. (2013), Kobayashi et al. (2009) and Ko et al. (2012) support the hypothesis that cyclin D2 plays an important role in PCa and could be a putative tumor suppressor. During this study the possible connection between an increased cyclin D2 expression and a proliferation inhibition in PCa ought to be further investigated. Thereby, the role of cyclin D2 in PCa is further elucidated by overexpression studies, downregulation studies and generation of a mouse model with a prostate epithelium-specific cyclin D2 knockout. 


\subsection{Aims of this study}

In the present study VPA was investigated as a putative candidate for PCa therapy. Thereby, especially the influence of VPA treatment on tumor and tumor cell angiogenesis was studied in vitro and in vivo. Furthermore, the role of the candidate gene cyclin D2 as a possible tumor suppressor gene in PCa was subject of this thesis.

The main aims were:

- Analyses of the molecular effects of VPA treatment on candidate gene expression

- Verification of deregulated candidate gene expression in mouse prostate tumor tissue

- Performance of an in vivo VPA treatment experiment with isolation of prostate tissue to verify deregulated candidate gene expression in mouse prostate tissue

- Verification of deregulated expression of one candidate gene in human PCa cell lines

- Analysis of the molecular effects of VPA treatment on angiogenesis

- In vitro expression analysis of markers for blood and lymphangiogenesis

- In vivo expression analysis of markers for blood and lymphangiogenesis

- Analysis of in vivo effects of VPA treatment on prostate tumor cells using the chicken chorioallantoic membrane (CAM) assay

- Performance of CAM experiments

- Macroscopic analysis of CAM tumors

- Determination of the CAM-tumor volume

- Immunohistological analysis of the CAM tumors by staining for the lymphatic vessel marker Prox1 and the blood vessel marker Mep21

- Expression analysis of markers for blood and lymphangiogenesis in CAM tumors

- Overexpression studies of cyclin D2

- Cloning of cyclin D2 into pIRES2-EGFP and pEBTetD

- Analysis of effects of transient cyclin D2 overexpression in human PCa cells on proliferation

- Generation of human PCa cells with stable and inducible overexpression of cyclin D2 
- Analysis of effects of stable and inducible cyclin D2 overexpression in human PCa cells on proliferation and migration

- Downregulation studies of cyclin D2

- Effects of siRNA-mediated cyclin D2 and cyclin D1 downregulation on migration behavior in NIH/3T3 cells

- Effects of siRNA-mediated cyclin D2 downregulation on transformation potential of $\mathrm{NIH} / 3 \mathrm{~T} 3$ cells

- Generation of a pSingle-tTs-Ccnd2-shRNA expression plasmid for inducible downregulation of cyclinD2

- Generation of NIH/3T3 cells with stable downregulation of cyclin D2

- Analysis of effects of stable cyclin D2 downregulation in NIH/3T3 cells on proliferation

- Analysis of effects of stable cyclin D2 downregulation in $\mathrm{NIH} / 3 \mathrm{~T} 3$ cells on transformation potential

- Analysis of functional consequences of cyclin D2 downregulation in VPA-treated PCa cells

- Generation of a conditional cyclin D2 knockout mouse model for cyclin D2 to study its physiological role in the prostate

- Genotyping and establishment of purchased mouse lines PB-Cre4 $4^{+}$and $\mathrm{CCnd}^{\mathrm{f} / \mathrm{fl}}$

- Mating of the two mouse lines to generate a conditional cyclin D2 knockout mouse model

- Verification of the prostate-specific deletion of cyclin D2

- Mating of conditional cyclin D2 knockout mice with TRAMP mice for studies on prostate tumor development and progression 


\section{Materials and Methods}

\subsection{Chemicals and Reagents}

\begin{tabular}{|c|c|}
\hline Chemicals/ Reagents & Manufacturer \\
\hline $\begin{array}{l}\text { 5-bromo-4-chloro-3-indolyl- } \beta \text {-D-galacto- } \\
\text { pyranoside galactopyranosid (X-Gal) } \\
\text { Adenosintriphosphate (ATP) }\end{array}$ & $\begin{array}{l}\text { Carl Roth } \mathrm{GmbH} \text {, Karlsruhe, Germany } \\
\text { Biomol GmbH, Hamburg, Germany }\end{array}$ \\
\hline Agar-Agar & Carl Roth GmbH, Karlsruhe, Germany \\
\hline Agarose & Carl Roth GmbH, Karlsruhe, Germany \\
\hline Ampicillin & Carl Roth GmbH, Karlsruhe, Germany \\
\hline Ampuwa & Fresenius AG, Bad Homburg, Germany \\
\hline Bacto-Trypton & Carl Roth GmbH, Karlsruhe, Germany \\
\hline Boric acid & Scharlau Chemie S.A., Barcelona, Spain \\
\hline Bromophenol blue & Carl Roth GmbH, Karlsruhe, Germany \\
\hline Cell culture media & $\begin{array}{l}\text { PAN, Aidenbach, Germany, Gibco by Life } \\
\text { Technologies, Darmstadt, Germany }\end{array}$ \\
\hline Crystal violet & $\begin{array}{l}\text { Sigma-Aldrich Chemie GmbH, Taufkirchen, } \\
\text { Germany }\end{array}$ \\
\hline Dimethylsulfoxid (DMSO) & Carl Roth GmbH, Karlsruhe, Germany \\
\hline Disodium phosphate $\left(\mathrm{Na}_{2} \mathrm{HPO}_{4}\right)$ & $\begin{array}{l}\text { Sigma-Aldrich Chemie GmbH, Taufkirchen, } \\
\text { Germany }\end{array}$ \\
\hline Dithiothreitol (DTT) & Biomol, Hamburg, Germany \\
\hline DHT (Dihydrotestosterone) & $\begin{array}{l}\text { Sigma-Aldrich Chemie GmbH, Taufkirchen, } \\
\text { Germany }\end{array}$ \\
\hline DNA Stain G & Serva GmbH, Heidelberg, Germany \\
\hline dNTPs (100 mM) & Life Technologies, Darmstadt, Germany \\
\hline Doxycycline-Hyclat Biochemica & AppliChem GmbH, Darmstadt, Germany \\
\hline $\begin{array}{l}\text { Dulbecco's phosphate buffered sodium } \\
\text { chloride solution (DPBS) }\end{array}$ & PAN, Aidenbach, Germany \\
\hline Ethanol & $\begin{array}{l}\text { Chemie Vertrieb Hannover, Hannover, } \\
\text { Germany }\end{array}$ \\
\hline Ethidiumbromid & $\begin{array}{l}\text { Sigma-Aldrich Chemie GmbH, Taufkirchen, } \\
\text { Germany }\end{array}$ \\
\hline Ethylendiamine-tetraacetic acid (EDTA) & ICN, Aurora, USA \\
\hline Ficoll@ 400 & AppliChem GmbH, Darmstadt, Germany \\
\hline Formaldehyde & Carl Roth GmbH, Karlsruhe, Germany \\
\hline Formamide & Carl Roth GmbH, Karlsruhe, Germany \\
\hline
\end{tabular}


Fluoromount-G

Fungizone

Geneticin disulfate (G418)-solution

Gentamycin sulphate solution

Glycerol

Glycin

L-Glutamine

Hydrochloric acid, J.T.Baker®

IGEPAL-CA-360 (NP-40)

Isopropanol

Kanamycin

Methanol

$\mathrm{NaOH}$

Natriumdeoxycholat

N.N-Dimethylformamide

NuPAGE ${ }^{\text {TM }}$ MES Running buffer (20x)

NuPAGE ${ }^{\text {TM }}$ LDS Sample buffer (4x)

NuPAGE ${ }^{T M}$ See Blue Plus2

OptiMEM®I

Orange-G

Penicillin/Streptomycin

peqGREEN DNA/RNA Dye

Potassium dihydrogen phosphate $\left(\mathrm{KH}_{2} \mathrm{PO}_{4}\right)$

Potassium acetate

Potassium chloride $(\mathrm{KCl})$

Powdered milk

Puromycin

Roti ${ }^{\circledR}$-Nanoquant

Simply Blue Safe Stain

Sodium chloride
Sigma-Aldrich Chemie GmbH, Taufkirchen, Germany

Life Technologies, Darmstadt, Germany

Carl Roth $\mathrm{GmbH}$, Karlsruhe, Germany

Carl Roth $\mathrm{GmbH}$, Karlsruhe, Germany

Carl Roth $\mathrm{GmbH}$, Karlsruhe, Germany

Carl Roth $\mathrm{GmbH}$, Karlsruhe, Germany

PAN, Aidenbach, Germany

Avantor Performance Materials B.V.,

Deventer, Netherlands

Sigma-Aldrich Chemie GmbH, Taufkirchen, Germany

Carl Roth $\mathrm{GmbH}$, Karlsruhe, Germany

Sigma Aldrich Chemie $\mathrm{GmbH}$, Taufkirchen, Germany

Carl Roth $\mathrm{GmbH}$, Karlsruhe, Germany

Merck KGaA, Darmstadt, Germany

AppliChem GmbH, Darmstadt, Germany

Sigma Aldrich Chemie $\mathrm{GmbH}$, Taufkirchen, Germany

Life Technologies, Darmstadt, Germany

Life Technologies, Darmstadt, Germany

Life Technologies, Darmstadt, Germany

PAN, Aidenbach, Germany

Sigma-Aldrich, Deisenhofen, Germany

PAN, Aidenbach, Germany

Peqlab Biotechnologie $\mathrm{GmbH}$, Erlangen, Germany

AppliChem GmbH, Darmstadt, Germany

Carl Roth $\mathrm{GmbH}$, Karlsruhe, Germany

Carl Roth $\mathrm{GmbH}$, Karlsruhe, Germany

Carl Roth $\mathrm{GmbH}$, Karlsruhe, Germany

InvivoGen, San Diego, USA

Carl Roth $\mathrm{GmbH}$, Karlsruhe, Germany

Life Technologies, Darmstadt, Germany

AppliChem GmbH, Darmstadt, Germany 
Sodiumdodecylsulfate (SDS)

Sucrose (D(+)-Saccharose)

Tris

Triton X-100

Tween 20

Valproic acid

VectaShield with DAPI

Xylencyanol

Yeast extract
Serva GmbH, Heidelberg, Germany

Carl Roth $\mathrm{GmbH}$, Karlsruhe, Germany

AppliChem GmbH, Darmstadt, Germany

Fluka, Deisenhofen, Germany

Carl Roth $\mathrm{GmbH}$, Karlsruhe, Germany

Sigma-Aldrich, Deisenhofen, Germany

VectorLab, Burlingame, USA

Sigma-Aldrich, Deisenhofen, Germany

Carl Roth $\mathrm{GmbH}$, Karlsruhe, Germany

\subsection{Biochemicals and enzymes}

\begin{tabular}{|c|c|}
\hline Biochemicals/enzymes & Manufacturer \\
\hline Albumin fraction $\mathrm{V}(\mathrm{BSA})$ & Biomol GmbH, Hamburg, Germany \\
\hline BigDye® & Life Technologies, Darmstadt, Germany \\
\hline $\begin{array}{l}\text { Complete Mini Protease Inhibitor Cocktail } \\
\text { Tablets }\end{array}$ & Roche, Mannheim, Germany \\
\hline DH5a TM Competent Cells & Life Technologies, Karlsruhe, Germany \\
\hline Direct PCR Lysis Reagent & Peqlab, Erlangen, Germany \\
\hline Fetal bovine serum (SeraPlus) & PAN, Aidenbach, Germany \\
\hline Immulase ${ }^{\mathrm{TM}}$ DNA Polymerase & Bioline, Luckenwalde, Germany \\
\hline Lipofectamine ${ }^{\circledR} 2000$ & $\begin{array}{l}\text { Thermo Fisher Scientific, Langenselbold, } \\
\text { Germany }\end{array}$ \\
\hline Matrige $(\circledast$ Basement Membrane Matrix & Corning Inc., New York, USA \\
\hline Metafectene $\AA^{\circledR}$ Pro & $\begin{array}{l}\text { Biontex Laboratories GmbH, Munich, } \\
\text { Germany }\end{array}$ \\
\hline MycoZap ${ }^{\mathrm{TM}}$ Spray & Lonza, Cologne, Germany \\
\hline Nu-Serum ${ }^{\mathrm{TM}}$ & Corning Life Sciences, Bedford, USA \\
\hline $\begin{array}{l}\text { Oligofectamine }{ }^{\mathrm{TM}} \text { Transfection Reagent } \\
\text { Phosnhatase Inhibitor Mix |l solution }\end{array}$ & $\begin{array}{l}\text { Thermo Fisher Scientific, Langenselbold, } \\
\text { Germany } \\
\text { Serva GmbH Heidelbera Germany }\end{array}$ \\
\hline $\begin{array}{l}\text { PolyFreeze (Tissue Freezing Medium) - } \\
\text { Clear } \\
\text { Platinum }{ }^{\circledR} \text { SYBR }^{\circledast} \text { Green qPCR SuperMix- } \\
\text { UDG with Rox } \\
\text { Proteinase K }\end{array}$ & $\begin{array}{l}\text { Polysciences Europe GmbH, Eppelheim, } \\
\text { Germany } \\
\text { Life Technologies, Darmstadt, Germany } \\
\text { Carl Roth GmbH, Karlsruhe, Germany }\end{array}$ \\
\hline ProteInase K & Carr Roin GmoH, Karısrune, Germany \\
\hline
\end{tabular}


Phusion $^{\mathrm{TM}}$ High-Fidelity DNA Polymerase

Restriction enzymes

Reverse Transcriptase SuperScript II

RNase A

T4 DNA Ligase

Tet System Approved Fetal Bovine Serum

Trypsin/EDTA solution
Finnzymes, Espoo, Finnland

New England Biolabs, Ipswich, USA

Life Technologies, Darmstadt, Germany AppliChem GmbH, Darmstadt, Germany

Life Technologies, Darmstadt, Germany Clontech-Takara, Saint Germain-en-Laye, France

PAN, Aidenach, Germany

\subsection{Usage ware}

Usage ware

10, 13, $50 \mathrm{ml}$ Cellstar ${ }^{\circledR}$ Tubes

6-, 12, 24- or 96well cell culture plates

96- well assay plates, white

384 well plates, white

384 well plates, black

4 chamber polystyrene vessel tissue culture treated glass slide

Amersham Hybond PVDF-Membran

Blotting Papier GB 002, 003, 004

Cell culture flasks (T25, T75)

Cover glass $24 \times 60 \mathrm{~mm}$

Cryo.S ${ }^{\mathrm{TM}}$ cups with screw cap

FALCON culture slides

Flat-bottomed Nucleon ${ }^{\mathrm{TM}}$ surface 96- well cell culture plates

Gloves

Membrane filter

Microcentrifuge tubes $(0.2 \mathrm{ml})$

Microscope slides Superfrost ${ }^{\circledR}$ Plus

Migration assay inserts

\section{Manufacturer}

Greiner-bio-one $\mathrm{GmbH}$, Frickenhausen, Germany

Sarstedt, Nümbrecht, Germany

Biozym, Hessisch Oldendorf, Germany

ABgene, Hamburg, Germany

4titude, Surrey, UK

Corning Life Sciences, Bedford, USA

GE Healthcare Life Sciences, Freiburg, Germany

Schleicher \& Schüll, Dassel, Germany

Sarstedt, Nümbrecht, Germany

Menzel Gläser, Braunschweig, Germany

Greiner-bio-one $\mathrm{GmbH}$, Frickenhausen, Germany

Becton Dickinson $\mathrm{GmbH}$, Heidelberg,

Germany

Nunc A/S, Roskilde, Denmark

Rösner- Mautby Meditrade $\mathrm{GmbH}$, Kiefersfelden, Germany Millipore, Billerica, USA

Sartedt, Nümbrecht, Germany

Schütt, Göttingen, Germany

Millipore, Billerica, USA, Corning Inc., New York, USA 
Needle Eclipse ${ }^{\mathrm{TM}}$ with Smartclip Technologie

Neubauer improved hemocytometer

Nunc® F96 Micro Well ${ }^{\mathrm{TM}}$ white

NuPAGE ${ }^{\mathrm{TM}} 4-12 \%$ Bis-Tris Gel

Pasteur pipette

Petri dishes

Petri dishes (cell culture), Nuncleon ${ }^{\mathrm{TM}}$ surface

Pipet tips

Pipettes (1000 $\mu \mathrm{l}, 200 \mu \mathrm{l}, 20 \mu \mathrm{l}, 10 \mu \mathrm{l})$

PVDF-Membrane

Quarz- Cuvette

Reaction tubes (1.5ml, $2 \mathrm{ml})$

Serological pipettes $(5 \mathrm{ml}, 10 \mathrm{ml})$

Sterile Single-use filter Minisart

Sterile surgical blades

Scissors (HSB-390-10/HSB-006-10)

Stainless steel beads $(\varnothing 5 \mathrm{~mm})$

Syringe Discardit ${ }^{\mathrm{TM}}$ II (10 $\left.\mathrm{ml} / 20 \mathrm{ml}\right)$

Syringe disposable filters, $0.45 \mu \mathrm{m} / 20 \mu \mathrm{m}$

Tweezer (Wironit HWC 110-10)

QPCR Adhesive Clear Seals
BD, Heidelberg, Germany

Hartenstein, Würzburg, Germany

Nunc A/S, Roskilde, Denmark

Life Technologies, Darmstadt, Germany

Brand GmbH\&Co.KG, Wertheim, Germany

Sarstedt, Nümbrecht, Germany

Nunc A/S, Roskilde, Denmark

Sarstedt, Nümbrecht, Germany

Gilson, Limburg-Offheim, Germany

GE Healthcare, Munich, Germany

Hellma, Mühlheim, Germany

Sartstedt, Nümbrecht, Germany

Sartstedt, Nümbrecht, Germany

Sartorius, Göttingen, Germany

Braun, Tuttlingen, Germany

Hammacher, Solingen, Germany

Quiagen, Hilden, Germany

BD, Heidelberg, Germany

Sartorius, Göttingen, Germany

Hammacher, Solingen,Germany/ Inox, Dumont, Switzerland

4titude, Surrey, UK

\subsection{Technical equipment}

\begin{tabular}{l|l}
\hline Technical equipment & Manufacturer \\
\hline 3500XL Genetic Analyzer & $\begin{array}{l}\text { Applied Biosystems GmbH, Darmstadt, } \\
\text { Germany } \\
\text { Applied Biosystems GmbH, Darmstadt, } \\
\text { System }\end{array}$ Autoclaves \\
Germany \\
Biomedis Laborservice GmbH, Gießen, \\
Webeco, Bad Schwartau, Germany \\
Systec, Wettenberg, Germany \\
Bio-Rad Laboratories GmbH, Munich, \\
Germany \\
Centrifuges (1-15, 1-15K, 4K15)
\end{tabular}


Centrifuge Heraeus Fresco21

$\mathrm{CO}_{2}$ Water Jacketed Incubator Series II

Electro Blotter (Fastblot B44)

Electrophoresis power supply Power Pac 3000

FluorChem ${ }^{\circledR} \mathrm{Q}$

Gel-image documentation system Doc-Print VX2

Heating block, MR Hei-Standard

Inverted microscope IX71

Fluorescence microscope BX60

Nanodrop 2000c

Mini centrifuge Sprout ${ }^{\mathrm{TM}}$

RM5 Assistent 348 Roller

Scale Quintix

Synergy Mx

Thermomixer 5436

TissueLyser LT

Thermocycler 2720

Vortexer

UV light table EXC-F20-M
Thermo Scientific, Langenselbold, Germany

Systec, Wettenberg

Biometra $\mathrm{GmbH}$, Göttingen, Germany

Bio-Rad Laboratories $\mathrm{GmbH}$, Munich, Germany

Alpha Innotech, Logan, Utah, USA

Vilber Lourmat, Eberhardzell, Germany

Heidolph Instruments, Schwabach, Germany

Olympus GmbH, Hamburg, Germany

Olympus $\mathrm{GmbH}$, Hamburg, Germany

Thermo Scientific, Langenselbold, Germany

Heathrow Scientific, Illinois, USA

Karl Hecht GmbH \& Co KG, Sondheim, Germany

Sartorius, Göttingen, Germany

Bio Tek, Bad Friedrichshall, Germany

Eppendorf AG, Hamburg, Germany

Qiagen, Hilden, Germany

Applied Biosystems, Carlsbad, USA

Schütt Labortechnik, Göttingen

Vilber Lourmat, Eberhardzell, Germany

\subsection{Sterilization of solutions and equipment}

Laboratory equipment, solutions and culture media were autoclaved at $121^{\circ} \mathrm{C}$ and $105 \mathrm{~Pa}$ for 60 min or sterilized at $220^{\circ} \mathrm{C}$ overnight.

\subsection{Ready-to-use reaction systems}

\begin{tabular}{l|l}
\hline Reaction system & Manufacturer \\
\hline $\begin{array}{l}\text { CellTiter } 96^{\circledR \quad A Q u a o u s ~ N o n-R a d i o a c t i v e ~} \\
\text { Proliferation Assay (MTS) }\end{array}$ & Promega, Mannheim, Germany \\
ECL Prime & GE Healthcare, Munich, Germany \\
Myco Alert@ Mycoplasma Detection Kit & Lonza, Cologne, Germany
\end{tabular}




\begin{tabular}{l|l} 
MSB® Spin PCR apace & Invitek, Berlin, Germany \\
NucleoSpin® Extract II & Machery \& Nagel, Düren. Germany \\
peqGold Total RNA Kit & PeqLab, Erlangen, Germany \\
PhosSTOP Phosphatase Inhibitor & Roche, Mannheim, Germany \\
PureLinkTM HiPure Plasmid Midiprep Kit & Life Technologies, Darmstadt, Germany \\
Diff-Quick Staining Set & Dade Behring GmbH, Marburg, Germany
\end{tabular}

\subsection{Solutions}

Solutions for routine applications were prepared according to Sambrook et al. (1989). Required chemicals were dissolved in $\mathrm{dd}_{2} \mathrm{O}$ and, when necessary, autoclaved or filtered under sterile conditions.

\begin{tabular}{|c|c|}
\hline Solution & Composition \\
\hline Blocking Buffer I (Western blot) & $\begin{array}{l}\text { 1x TBS-Tween } \\
5 \% \text { low-fat dry milk }\end{array}$ \\
\hline Antibody solution (Immunohistochemistry) & $\begin{array}{l}1 \mathrm{x} \text { TBS }(0.05 \mathrm{M}, \mathrm{pH} 7.2-7.4) \\
1 \% \text { BSA } \\
0.5 \% \text { Triton X-100 }\end{array}$ \\
\hline Blocking Buffer II (Immunohistochemistry) & 1x PBS with $3 \%$ BSA \\
\hline $\begin{array}{l}\text { Goat blocking reagent (Immuno- } \\
\text { histochemistry) }\end{array}$ & $\begin{array}{l}1 \times \text { PBS } \\
5 \% \text { goat serum } \\
1 \% \text { BSA } \\
0.2 \% \text { Triton } X-100\end{array}$ \\
\hline Lysis Buffer for Protein (modified RIPA) & $\begin{array}{l}150 \mathrm{mM} \mathrm{NaCl} \\
1 \mathrm{mM} \text { EDTA } \\
50 \mathrm{mM} \text { Tris-HCl, pH } 7.4 \\
1 \% \text { IGEPAL-CA-360 (NP-40) } \\
0.25 \% \text { sodium deoxycholate } \\
1 \text { Tablet/10 ml Complete-Mini protease- } \\
\text { inhibitor } \\
100 \mu \mathrm{l} / 10 \mathrm{ml} \text { Phosphatase-Inhibitor-Mix II } \\
\text { solution }\end{array}$ \\
\hline
\end{tabular}




\begin{tabular}{|c|c|}
\hline P1 Buffer (Plasmid preparation) & $\begin{array}{l}50 \text { mM Tris- } \mathrm{HCl}, \mathrm{pH} 8.0 \\
10 \mathrm{mM} \text { EDTA } \\
100 \mu \mathrm{g} / \mathrm{ml} \text { RNase A }\end{array}$ \\
\hline P2 Buffer (Plasmid preparation) & $\begin{array}{l}200 \mathrm{mM} \mathrm{NaOH} \\
1 \% \mathrm{SDS}\end{array}$ \\
\hline P3 Buffer (Plasmid preparation) & 3 M Potassium acetate, $\mathrm{pH} 5.5$ \\
\hline 10x PBS & $\begin{array}{l}1.37 \mathrm{M} \mathrm{NaCl} \\
81 \mathrm{mM} \mathrm{Na}_{2} \mathrm{HPO}_{4} \\
27 \mathrm{mM} \mathrm{KCl} \\
14.7 \mathrm{mM} \mathrm{KH}_{2} \mathrm{PO}_{4}\end{array}$ \\
\hline 10x TBS & $\begin{array}{l}1.37 \mathrm{M} \mathrm{NaCl} \\
100 \mathrm{mM} \text { Tris } \\
\text { Adjust to } \mathrm{pH} 7.6 \text { with } \mathrm{HCl}\end{array}$ \\
\hline 1x TBS-Tween (TBS-T) & $\begin{array}{l}10 \% 10 x \text { TBS } \\
0.1 \% \text { Tween } 20\end{array}$ \\
\hline Stop Mix I & $\begin{array}{l}95 \% \text { Formamide } \\
20 \text { mM EDTA } \\
0.05 \% \text { Bromophenol blue } \\
0.05 \% \text { Xylencyanol }\end{array}$ \\
\hline Stop Mix II & $\begin{array}{l}15 \% \text { Ficoll } 400 \\
200 \mathrm{mM} \text { EDTA } \\
0.1 \% \text { Orange G }\end{array}$ \\
\hline Transfer Buffer Ila (Western blot) & $\begin{array}{l}25 \mathrm{mM} \text { Tris pH } 8.3 \\
150 \mathrm{mM} \text { Glycin } \\
20 \% \text { Methanol }\end{array}$ \\
\hline 20x Turbo-Puffer & $\begin{array}{l}0.2 \mathrm{M} \mathrm{NaOH} \\
\text { Adjust to } \mathrm{pH} 8.0 \text { with } \mathrm{H}_{3} \mathrm{BO}_{3} \text { (Boric acid) }\end{array}$ \\
\hline Washing Buffer I (Western blot) & $\begin{array}{l}\text { 1x TBS-Tween } \\
2.5 \% \text { low-fat dry milk }\end{array}$ \\
\hline X-Gal stock solution & $20 \mathrm{mg} \mathrm{X-Gal/ml} \mathrm{N.N-Dimethylformamide}$ \\
\hline
\end{tabular}




\subsection{Culture media, antibiotics, agar plates}

\subsubsection{Culture media for bacteria}

Luria-Bertani medium (LB medium), $\mathrm{pH} 7.0$ : $\quad 1 \%$ Bacto-Trypton

$0.5 \%$ Yeast extract

$1 \% \mathrm{NaCl}$

The LB medium was prepared with bi-distilled water. Afterwards it was autoclaved and kept at $4{ }^{\circ} \mathrm{C}$. For selection, either ampicillin $(50 \mu \mathrm{g} / \mathrm{ml}$ final concentration) or kanamycin $(25 \mu \mathrm{g} / \mathrm{ml}$ final concentration) was added to the medium.

\subsubsection{Agar plates}

Agar plates were prepared by adding $1.5 \%$ (w/v) Agar-Agar to the liquid LB medium before autoclaving. Following the autoclaving procedure the LB medium was cooled to $55^{\circ} \mathrm{C}$ on a stirring plate before the antibiotics were added in the corresponding concentration (ampicillin: 50 $\mu \mathrm{g} / \mathrm{ml}$, kanamycin: $25 \mu \mathrm{g} / \mathrm{ml})$. Finally, the medium was poured into petri dishes and set aside to cool out and harden. The ready-to-use agar plates were stored in a sterile plastic back at $4^{\circ} \mathrm{C}$.

\subsubsection{Media for eukaryotic cell cultures}

Media used for the culture of eukaryotic cells was purchased from PAN, Aidenbach, Germany. Before use, fetal bovine serum (FBS), which was heat-inactivated for 30 min at $56{ }^{\circ} \mathrm{C}$, and antibiotics (Penicillin/Streptomycin) were added. The following media were used for cell culture:

\begin{tabular}{l|l}
\hline Cell line & Components of the media \\
\hline $2 \mathrm{E}$ & Advanced DMEM \\
& $8 \%$ NuSerum \\
& $10 \%$ FBS \\
& $100 \mathrm{nM}$ Dihydrotestosterone (DHT) \\
& $80 \mu \mathrm{g} / \mathrm{ml}$ Gentamycin \\
$1 \%$ Glutamine
\end{tabular}




\begin{tabular}{|c|c|}
\hline Caco-2 & $\begin{array}{l}\text { MEM } \\
20 \% \text { FBS } \\
1.2 \% \text { Penicillin/Streptomycin }\end{array}$ \\
\hline LNCaP, PC-3, DU145, SW620 & $\begin{array}{l}\text { RPMI } 1640 \\
10 \% \text { FBS } \\
1.2 \% \text { Penicillin/Streptomycin }\end{array}$ \\
\hline LNCaP and PC-3 + pIRES2-EGFP-CCND2 & $\begin{array}{l}\text { See LNCaP, PC-3 } \\
+400 \mu \mathrm{g} / \mathrm{ml} \mathrm{G} 418\end{array}$ \\
\hline LNCaP + pEBTetD-CCND2 & $\begin{array}{l}\text { RPMI } 1640 \\
10 \% \text { Tet System approved FBS } \\
1.2 \% \text { Penicillin/Streptomycin } \\
0.4 \mu \mathrm{g} / \mathrm{ml} \text { Puromycin }\end{array}$ \\
\hline PC-3 + pEBTetD-CCND2 & $\begin{array}{l}\text { RPMI } 1640 \\
10 \% \text { Tet System approved FBS } \\
1.2 \% \text { Penicillin/Streptomycin } \\
1.4 \text { mg/ ml Puromycin }\end{array}$ \\
\hline $\mathrm{NIH} / 3 \mathrm{T3}$ & $\begin{array}{l}\text { DMEM } \\
10 \% \text { FBS } \\
1.2 \% \text { Penicillin/Streptomycin }\end{array}$ \\
\hline NIH/3T3 + pSingle-tTs-Ccnd2-shRNA & $\begin{array}{l}\text { See NIH/3T3 } \\
+400 \mu \mathrm{g} / \mathrm{ml} \text { G418 }\end{array}$ \\
\hline
\end{tabular}

For cryopreservation of the cells in liquid nitrogen cryo-medium was diluted 1:1 with the appropriate medium.

Cryo-medium: $\quad 7.5 \mathrm{ml}$ medium of the corresponding cell line

$+12.5 \mathrm{ml} \mathrm{FBS}$

$+5 \mathrm{ml} \mathrm{DMSO}$

The solution was sterile filtered with a $20 \mu \mathrm{M}$ filter, aliquoted and stored at $-20^{\circ} \mathrm{C}$. 


\subsection{Biologic material}

\subsubsection{Bacterial strains}

The bacterial strain Escherichia coli DH5a was used for the transformation of plasmids (Hanahan 1983). It was purchased form Life Technologies, Karlsruhe, Germany.

\subsubsection{Eukaryotic cell lines}

\begin{tabular}{|c|c|}
\hline Cell line & Description \\
\hline $2 \mathrm{E}$ & $\begin{array}{l}\text { Murine prostate adenocarcinoma cell line, generated from a } \\
\text { prostate tumor of a TRAMP mouse in our research group, C57/BI6 } \\
\text { background, (Hardenberg, 2010) }\end{array}$ \\
\hline CaCo-2 & $\begin{array}{l}\text { Human colorectal adenocarcinoma cell line, ATCC, Rockville, } \\
\text { USA }\end{array}$ \\
\hline DU145 & $\begin{array}{l}\text { Human prostate adenocarcinoma cell line (brain metastasis), } \\
\text { castration resistant (Stone et al. 1978) }\end{array}$ \\
\hline LNCaP & $\begin{array}{l}\text { Human prostate adenocarcinoma cell line (lymph node } \\
\text { metastasis), androgen dependent (Horoszewicz et al. 1983), } \\
\text { ATCC, Rockville, USA }\end{array}$ \\
\hline $\mathrm{NIH} / 3 \mathrm{T3}$ & Murine embryonal fibroblast cell line, ATCC, Rockville, USA \\
\hline PC-3 & $\begin{array}{l}\text { Human prostate adenocarcinoma cell line (bone metastasis), } \\
\text { castration resistant (Kaighn et al. 1979, Ohnuki et al. 1980), } \\
\text { ATCC, Rockville USA }\end{array}$ \\
\hline SW 620 & $\begin{array}{l}\text { Human colorectal adenocarcinoma cell line (lymph node } \\
\text { metastasis), ATCC, Rockville, USA }\end{array}$ \\
\hline
\end{tabular}

\subsubsection{Mouse strains}

All mice experiments were conducted according to the European and German protection of animals act. The number of sacrificed mice was kept to a minimum as well as the stress and pain level. The mice were euthanized by $\mathrm{CO}_{2}$-asphyxation and subsequent cranial dislocation. Keeping conditions were set to 12 hours light/dark cycles at $22^{\circ} \mathrm{C}$ and $55 \pm 5 \%$ relative humidity. Animal food was ordered from ssniff Spezialdiäten $\mathrm{GmbH}$, Soest, Germany. 
TRAMP mice with a C57/BI6 background were ordered and obtained from Jackson Lab. (Bar Harbor, USA). C57/BI6 mice came from a colony of our own department.

Heterozygous Ccnd $2^{f l o x /+}$ mice were kindly provided by Peter Sicinski from the Dana Faber Institute, Boston, USA. PB-Cre $4^{+}$mice were kindly provided by Xiantuo Wu from the Department of Pathology, Keck School of Medicine, University of Southern California, USA (Wu et al. 2001).

\subsubsection{Synthetic DNA oligonucleotides}

For the generation of PCR products as well as for quantitative RT-PCR (qRT-PCR) analysis synthetic oligonucleotides were purchased from Eurofins MWG Operon (Ebersberg, Germany). Sequences are listed from the 5'- to $3^{\prime}$ - end.

Human specific primers for quantitative real-time PCR

\begin{tabular}{l|l}
\hline Primer name & Sequence \\
\hline Cp-Q1-fw & CTCTGATCACCCCGAGAAAG \\
Cp-Q1-rev & AAGCTATGGCCGTGAAAATG \\
\hline Cp-Q2-fw & AAACAATTTACTCTTGTGCAACAC \\
Cp-Q2-rev & GGAATGTTCCGTGTCAACAG \\
\hline Cyclin D2-Q1-fw & ATTGCTCTGTGTGCCACCGACTT \\
Cyclin D2-Q1-rev & CCGTCACGTTGGTCCTGACGG \\
\hline GAPDH-fw & CATCACCATCTTCCAGGAGC \\
GAPDH-rev & ATGACCTTGCCCACAGCCTT \\
\hline HPRT-fw & ACCCTTTCCAAATCCTCAGC \\
HPRT-rev & GTTATGGCGACCCGCAG \\
\hline LDHA-fw & GGAGATCCATCATCTCTCCC \\
LDHA-rev & GGCCTGTGCCATCAGTATCT \\
\hline PBGD-fw & GCAATGCGGCTGCAACGGCGGAAG \\
PBGD-rev & CCTGTGGTGGACATAGCAATGATT \\
\hline TBP-fw & AGCCTGCCACCTTACGCTCAG \\
TBP-rev & TGCTGCCTTTGTTGCTCTTCCA \\
\hline VEGFA-Q1-fw & AAGGAGGAGGGCAGAATCAT \\
VEGFA-Q1-rev & GCAGTAGCTGCGCTGATAGA \\
\hline VEGFC-Q1-fw & TGAACACCAGCACGAGCTAC \\
VEGFC-Q1-rev & GCCTTGAGAGAGAGGCACTG \\
\hline VEGFR1-Q1-fw & TCCAAGAAGTGACACCGAGA \\
VEGFR-Q1-rev & TTGTGGGCTAGGAAACAAGG \\
\hline VEGFR2-Q1-fw & GACTTGGCCTCGGTCATTTA \\
VEGFR2-Q1-rev & ACACGACTCCATGTTGGTCA \\
\hline
\end{tabular}


sVEGFR1-Q1-fw

sVEGFR1-Q1-rev

sVEGFR2-Q1-fw

sVEGFR2-Q1-rev
GCACGTTTGGATTTGGAGGA

GGAAAGGATCATCCCAAGTTGTT

GCCTTGCTCAAGACAGGAAG

CAACTGCCTCTGCACAATGA

Mouse specific primers for quantitative real-time PCR

\begin{tabular}{|c|c|}
\hline Primer name & \\
\hline Ang1-Q1-fw & CATTCTTCGCTGCCATTCTG \\
\hline Ang1-Q1-rev & GCACATTGCCCATGTTGAATC \\
\hline Ccnd1-Q1-fw & ACACCAGCTCCTGTGCTGCGAA \\
\hline Ccnd1-Q1-rev & CCAGGTAGTTCATGGCCAGCGG \\
\hline Ccnd2-Q1-fw & GGAGCTGCTGGAGTGGGAACTGGT \\
\hline Ccnd2-Q1-rev & GCGCATGCTTGCGGATCAGGGACA \\
\hline Cp1-Q1-fw & ACCAAGCAGGGCCTGGGAAAAGGA \\
\hline Cp1-Q1-rev & CCCAAGTGCTCGTCTTCGGCTCGT \\
\hline Cp2-Q1-fw & TACTCCACTGCCACGTGACTGACC \\
\hline Cp2-Q1-rev & ACCAGGTCCCTCGCAAATGAACAGT \\
\hline Cxcl15-Q1-fw & GGCTGTCCTTAACCTAGGCATCTT \\
\hline Cxcl15-Q1-rev & AGCATCAGGATCCAAACAAATCAT \\
\hline HPRT-fw & AGCCCCAAAATGGTTAAGGTTGC \\
\hline HPRT-rev & TTGCAGATTCAACTTGCGCTCAT \\
\hline Lif1-Q1-fw & AGACTGTGGAGGGCTGCGAGACCA \\
\hline Lif1-Q1-rev & ATGGGTGGCGTATGGCACAGGTGG \\
\hline PECAM-1-Q1-fw & GAGCCCAATCACGTTTCAGTTT \\
\hline PECAM-1-Q1-rev & TCCTTCCTGCTTCTTGCTAGCT \\
\hline Ptprn-Q1-fw & TGCCCACGGCTGTCTGTTTGACCG \\
\hline Ptprn-Q1-rev & TGGGCACCAAACCAGACCTGTCCC \\
\hline Rcbtb2-Q1-fw & TCCAGCGGGTTGCCTGTGGCTACG \\
\hline Rcbtb2-Q1-rev & TGCCCACCTTGTGTCTTGGCAGCA \\
\hline Tie-1-Q3-fw & TCAACTGCAGCTCCAAAATG \\
\hline Tie-1-Q3-rev & CATGACAGACACCTCCATGC \\
\hline TBP-Q-fw & CACCAATGACTCCTATGACCCCTA \\
\hline TBP-Q-rev & CAGTTGTCCGTGGCTCTCTTATTC \\
\hline Uchl1-Q1-fw & GTACGAGCTCGATGGGCGAATGCC \\
\hline Uchl1-Q1-rev & GGGATCGGCTGGTTCTCTCTCCCC \\
\hline VEGFA-Q1-fw & CCC CGG ACG GGC CTC CGA AA \\
\hline VAGFA-Q1-rev & TGC ACA GCG CAT CAG CGG CA \\
\hline VEGFA-Q2-fw & GGAGATCCTTCGAGGAGCACTT \\
\hline VEFGA-Q2-rev & GGCGATTTAGCAGCAGATATAAGAA \\
\hline VEGFC-Q1-fw & TTCTTGTCTCTGGCGTGTTCCC \\
\hline VEGFC-Q1-rev & GCTCCTCCAGGTCTTTGCCTTC \\
\hline
\end{tabular}


VEGFR1/FIt-1-Q2-fw VEGFR1/FIt-1-Q2-rev VEGFR2/KDR-Q1-fw VEGFR2/KDR-Q1-rev
CAAGAGCGATGTGTGTCCT TCCCATCCTGTTGGACGTTG GCCCTGCTGTGGTCTCACTAC CAAAGCATTGCCCATTCGAT

Cyclin D2 shRNA oligonucleotides for cloning into pSingle-tTs-shRNA

\begin{tabular}{l|l}
\hline Primer name & Sequence \\
\hline shRNA-B-fw & $\begin{array}{l}\text { ctcgagGCCACACTGATGTGGATTGTCTCAAATTCAAGAGA } \\
\text { TTTGAGACAATCCACATCAGTGTGGTTTTTTACGCGTa } \\
\text { shRNA-B-rev } \\
\text { aagcttACGCGTAAAAAACCACACTGATGTGGATTGTCTCAA } \\
\text { ATCTCTTGAATTTGAGACAATCCACATCAGTGTGGCc }\end{array}$ \\
shRNA-C-fw & $\begin{array}{l}\text { ctcgagGTGCTGGAGTGGGAACTGGTAGTGTTTTCAAGAGA } \\
\text { AACACTACCAGTTCCCACTCCAGCATTTTTTACGCGTa } \\
\text { aagcttACGCGTAAAAATGCTGGAGTGGGAACTGGTAGTG } \\
\text { TTTCTCTTGAAACACTACCAGTTCCCACTCCAGCACc }\end{array}$
\end{tabular}

Primers for amplification of cDNA designated for cloning

\begin{tabular}{l|l}
\hline Primer name & Sequence \\
\hline CyclinD2-fw-Kpn (pEBTetD) & GGTACCATGGAGCTGCTGTGCCACGAGGTGG \\
\hline $\begin{array}{l}\text { CyclinD2-rev-EcoRI (pEBTetD/pIRES2- } \\
\text { EGFP) }\end{array}$ & GAATTCTCACAGGTCGATATCCCGCACGTCTG \\
\hline CyclinD2-fw-Xhol (pIRES2-EGFP) & CTCGAGATGGAGCTGCTGTGCCACGAGGTGG
\end{tabular}

Mouse specific primers for genotyping

\begin{tabular}{l|l}
\hline Primer name & Sequence \\
\hline Cyclin D2 flox 3' & CAGTCCTCCAGCACACCA \\
Cyclin D2 flox 5 & GAGGACGAGTTTGTGCTCA \\
Cyclin D2 delta & CCAGACTTTATCCTCCGGGT \\
\hline PB-Cre Ell a cre fw & CCTGGAAAATGCTTCTGTCCG \\
PB-Cre Ell a cre rev & CAGGGTGTTATAAGCAATCCC \\
\hline TRAMP rPB-423 fw & CTCTGCACCTTGTCAGTGAGGTCCAG \\
TRAMP-SV40Tag rev & CTCCTTTCAAGACCTAGAAGGTCCA
\end{tabular}




\subsubsection{Synthetic RNA oligonucleotides}

Synthetic mouse RNA oligonucleotides (siRNAs)

\begin{tabular}{l|l}
\hline Primer name & Sequence \\
\hline Cyclin D2 A & CAGCAGUUCCGUCAAGAGCAGCAUA \\
\hline Cyclin D2 B & UAUGCUGCUCUUGACGGAACUGCUG \\
\hline Cyclin D2 C & CCACACUGAUGUGGAUUGUCUCAAA \\
\hline Luciferase & CGUACGCGGAAUACUUTGATT
\end{tabular}

Synthetic human RNA oligonucleotides (siRNAs)

\begin{tabular}{l|l}
\hline Primer name & Sequence \\
\hline Cyclin D2 A & UGCUCCUCAAUAGCCUGCAGCAGUA \\
\hline Cyclin D2 B & UGACGGAUCCAAGUCGGAGGAUGAA \\
\hline Cyclin D2 C & GAUGAGGAAGUGAGCUCGCUCACUU
\end{tabular}

Vector specific primers for sequencing

\begin{tabular}{l|l}
\hline Primer name & Sequence \\
\hline Sp6-new & TTAGGTGACACTATAGAATACTCAAGC \\
\hline T7-new & AATACGACTCACTATAGGGCGAATTGG \\
\hline pSingle-fw & GCCATGAACAAAGGTGGCTATAAAGAGGTCATC \\
pSingle-rev & GAAGCGGAAGAGCGCCCAATACGCAAACCGCCT \\
\hline pIRES2-EGFP-3fw & AGACCTTCATTGCTCTGTGTGC \\
pIRES2-EGFP-4fw & CTTCCCTCTGGCCATGAATTAC \\
pIRES2-EGFP-3rev & TTCGAAGCTTGAGTCACAGGTC \\
pIRES2-EGFP-5rev & ATAGACAAACGCACACCGGCCTTATTC \\
\hline pEBTetD-fw & GTGAACCGTCAGATCGCCTG \\
pEBTetD-rev & CGTGTCACATGTGGAACAGG
\end{tabular}




\subsubsection{Antibodies}

\subsubsection{Primary antibodies}

\begin{tabular}{|c|c|c|}
\hline Antibody & Manufacturer & Dilution \\
\hline $\begin{array}{l}\text { Anti- } \alpha-\text { Tubulin (clone B-5-1- } \\
\text { 2), monoclonal antibody, } \\
\text { mouse }\end{array}$ & Sigma-Aldrich, Deisenhofen, Germany & $1: 10000$ \\
\hline $\begin{array}{l}\text { Anti-Ceruloplasmin } \\
\text { (ab135649), polyclonal } \\
\text { antibody, rabbit }\end{array}$ & Abcam, Cambridge, UK & $1: 2000$ \\
\hline $\begin{array}{l}\text { Anti-Cyclin D1 (A-12), } \\
\text { monoclonal antibody, mouse }\end{array}$ & Santa Cruz, Heidelberg, Germany & $1: 2000$ \\
\hline $\begin{array}{l}\text { Anti-Cyclin D2 (M-20), } \\
\text { polyclonal antibody, rabbit }\end{array}$ & Santa Cruz, Heidelberg, Germany & $1: 200$ \\
\hline $\begin{array}{l}\text { Anti-Cyclin D2 (D52F9), } \\
\text { monoclonal antibody, rabbit }\end{array}$ & Cell Signaling, Danvers, USA & $1: 2000$ \\
\hline $\begin{array}{l}\text { Anti-HSC-70 (B-6), } \\
\text { monoclonal antibody, mouse }\end{array}$ & Santa Cruz, Heidelberg, Germany & $1: 10000$ \\
\hline Anti-Mep21, mouse & $\begin{array}{l}\text { M. Williams, AbLab, University of } \\
\text { British Columbia, Vancouver, B.C., } \\
\text { Canada }\end{array}$ & $1: 500$ \\
\hline $\begin{array}{l}\text { Anti-Prox1 (102-PA32), } \\
\text { polyclonal antibody, rabbit }\end{array}$ & $\begin{array}{l}\text { ReliaTech GmbH, Wolfenbüttel, } \\
\text { Germany }\end{array}$ & $1: 500$ \\
\hline
\end{tabular}

\subsubsection{Secondary antibodies}

\begin{tabular}{l|l|l}
\hline Antibody & Manufacturer & Dilution \\
\hline $\begin{array}{l}\text { Anti-rabbit IgG, } \\
\text { Cy3-conjugated, sheep }\end{array}$ & Sigma-Aldrich, Deisenhofen & $1: 200$ \\
\hline $\begin{array}{l}\text { Anti-rabbit IgG (H+L), HRP- } \\
\text { (horse radish peroxidase) } \\
\text { conjugated, goat }\end{array}$ & $\begin{array}{l}\text { Dianova Jackson ImmunoResearch, } \\
\text { Hamburg, Germany }\end{array}$ & $1: 5000$ \\
\hline $\begin{array}{l}\text { Anti-mouse IgG1 (v1), Alexa } \\
\text { Fluor® 488-conjugated, goat }\end{array}$ & $\begin{array}{l}\text { Life Technologies, Darmstadt, } \\
\text { Germany }\end{array}$ & $1: 200$ \\
\hline $\begin{array}{l}\text { Anti-rabbit IgG, Alexa Fluor® } \\
\text { 594-conjugated, goat }\end{array}$ & $\begin{array}{l}\text { Life Technologies, Darmstadt, } \\
\text { Germany }\end{array}$ & $1: 200$ \\
\hline $\begin{array}{l}\text { Anti-mouse IgG (H+L), HRP- } \\
\text { conjugated, rabbit }\end{array}$ & $\begin{array}{l}\text { Dianova, Jackson ImmunoResearch, } \\
\text { Hamburg, Germany }\end{array}$ & $1: 5000$ \\
& $1: 10000$
\end{tabular}




\subsubsection{Plasmids and Vectors}

\begin{tabular}{l|l}
\hline Plasmid/Vector & Source \\
\hline pGEM®-T Easy & Promega, Wisconsin, USA \\
\hline pIRES2-EGFP & $\begin{array}{l}\text { Clontech-Takara, Saint Germain-en-Laye, } \\
\text { France }\end{array}$ \\
\hline pIRES2-EGFP-CCND2 & $\begin{array}{l}\text { Claudia Morich, 2013 } \\
\text { Bach et al. 2007 }\end{array}$ \\
\hline pEBTetD & Claudia Morich, 2013 \\
\hline pEBTetD-CCND2 & $\begin{array}{l}\text { Clontech-Takara, Saint Germain-en-Laye, } \\
\text { France }\end{array}$ \\
\hline pSingle-tTs-shRNA & Claudia Morich, 2014 \\
\hline pSingle-tTs-shRNA-Ccnd2 & $\begin{array}{l}\text { Clontech-Takara, Saint Germain-en-Laye, } \\
\text { France }\end{array}$ \\
\hline pEGFP & Silke Kaulfuß (unpublished data) \\
\hline pEGFP-KRAS & Silke Kaulfuß (unpublished data) \\
\hline pEGFP-KRAS-G12V &
\end{tabular}

\subsection{Databases}

\begin{tabular}{l|l}
\hline Usage & Program \\
\hline Analysis of DNA and protein sequences & $\begin{array}{l}\text { BLAST-program, (Altschul et al. 1990) } \\
\text { (http://www.ncbi.nlm.nih.gov) }\end{array}$ \\
\hline Restriction site analysis & $\begin{array}{l}\text { NEB Cutter 2.0 } \\
\text { (http://tools.neb.com/NEBcutter2/index.php) } \\
\text { WEB Cutter 2.0 } \\
\text { (http://rna.lundberg.gu.se/cutter2) }\end{array}$ \\
\hline Bioinformatics & $\begin{array}{l}\text { Ensembl v32 (http://www.ensembl.org) } \\
\text { National Center for Biotechnology } \\
\text { Information (http://ncbi.nlm.nih.gov) }\end{array}$ \\
\hline Primer design & $\begin{array}{l}\text { Primer3 (http://bioinfo.ut.ee/primer3-0.4.0) } \\
\text { Erimer-BLAST } \\
\text { (http://www.ncbi.nlm.nih.gov/tools/primer- } \\
\text { blast/index.cgi?LINK_LOC=BlastHome) }\end{array}$ \\
\hline Statistical analysis & $\begin{array}{l}\text { GraphPad } \\
\text { (http://graphpad.com/quickcalcs/ttest1.cfm) }\end{array}$ \\
\hline
\end{tabular}




\subsection{Isolation, purification and concentration determination of nucleic acids}

\subsubsection{Isolation of total RNA from cell cultures}

Total RNA from cell cultures was isolated and purified by using the peqGOLD Total RNA Kit according to the manufactures instruction (Peqlab, Erlangen, Germany).

\subsubsection{Isolation of RNA from mouse tissue}

RNA from mouse prostate or prostate tumor tissue was obtained via homogenization of the tissue using the TissueLyser LT (Quiagen, Hilden, Germany). A small sample of the tissue was transferred to a $2 \mathrm{ml}$ reaction tube together with a stainless steel bead ( $\varnothing 5 \mathrm{~mm}$, Quiagen) and $400 \mu \mathrm{l}$ RNA lysis buffer T from the peqGOLD Total RNA Kit (Peqlab, Erlangen, Germany). The amount of used RNA lysis buffer T depended on the size of the tissue sample. The tissue was homogenized for $5 \mathrm{~min}$ at 50 1/s oscillation in the TissueLyser LT. Thereafter the cups were kept for $5 \mathrm{~min}$ on ice before centrifuging them for $10 \mathrm{~min}$ at $13000 \mathrm{rpm}$ and $4^{\circ} \mathrm{C}$. The supernatant containing the lysed cells was transferred to a new $1.5 \mathrm{ml}$ reaction tube and RNA isolation and purification was continued by use of the peqGOLD Total RNA Kit according to the manufactures instruction.

\subsubsection{Determination of nucleic acid concentration}

The concentration of nucleic acids was measured by either one of three different methods. One approach was to use a spectral photometer (BioPhotometer, Eppendorf, Germany). The blank value was measured with water or whatever other substance the nucleic acid was eluted in. Then the absorption maximum of nucleic acids $(260 \mathrm{~nm})$ was measured. Contaminations by proteins $(280 \mathrm{~nm})$ or salts $(320 \mathrm{~nm})$ were also detected. The nucleic acid concentration was calculated by the Lambert's law. A Nanodrop-photometer (PeqLab, Erlangen, Germany) was also used to measure the concentration of nucleic acids, especially of RNAs. The blank value was measured similar to the spectral photometer with water or whatever other substance the nucleic acid was eluted in. Only $1 \mu$ of the sample is needed to measure the absorption maximum of nucleic acids $(260 \mathrm{~nm})$. Another method used to measure the nucleic acid concentration, especially of low concentrations, was by agarose gel electrophoresis. $2 \mu$ of DNA were loaded onto an agarose gel and run with the MassRuler DNA standard (MassRuler DNA ladder, Thermo Scientific, Langenselbold, Germany). The ladder consists of a mixture of 
chromatography-purified individual DNA fragments and therefore the amount of DNA for each band was known. A picture was taken of the stained DNA when the gel was run for an appropriate time. The DNA concentration of the samples was based upon the band intensities.

\subsection{Cloning techniques}

\subsection{1 pSingle-tTs-shRNA cloning}

Cyclin D2 shRNA oligonucleotides were designed using the shRNA Sequence Designer software (Clontech) and purchased. The shRNA oligonucleotides were annealed and cloned into the Xhol/Hindlll-digested pSingle-tTS-shRNA vector according to the manufacturer instruction (Knockout ${ }^{\mathrm{TM}}$ Single Vector Inducible RNAi System, Clontech-Takara, Saint Germain-en-Laye, France).

\subsubsection{Amplification of DNA for subcloning into $p G E M{ }^{\circledR}-T$ Easy vector}

cDNA generated from RNA of either mouse or human cell lines was used for amplification of the open reading frame of the gene of interest using specific primers. The PCR reaction was conducted according to section 2.14.2.

\subsubsection{Purification of the PCR-product}

The generated PCR product was purified using the MSB® Spin PCR apace Kit (Invitek, Berlin, Germany). The purification was done according to the manufacturer's instruction.

\subsubsection{Subcloning of PCR products/ TA-cloning}

Many polymerases, including the Taq DNA polymerase, possess a terminal transferase activity which results in the non-template addition of a single nucleotide to the $3^{\prime}$-ends of the PCR products (Clark 1988, Hu 1993). The Phusion ${ }^{\text {TM }}$ High-Fidelity DNA polymerase (Finnzymes, Finnland) contains no such terminal transferase activity. Therefore, PCR products amplified with the Phusion ${ }^{\mathrm{TM}}$ High-Fidelity DNA polymerase contain no 3'-overhangs. Since the pGEM®-T Easy vector, which contains 5'-dT overhangs, was used for cloning of PCR products, the terminal $\mathrm{dA}$ had to be added to the PCR products. The following A-tailing reaction was carried out before the PCR product could be cloned into the pGEM®-T Easy vector (TA cloning): 
$7 \mu \mathrm{l} \mathrm{PCR}$ product (purified)

$1 \mu \mathrm{l} 1 \mathrm{x}$ Immolase buffer

$0.3 \mu \mathrm{MgCl} 2$

$1 \mu \mathrm{l}$ Immolase

$2 \mu \mathrm{d}$ ATPs $(2 \mathrm{mM})$

The reaction mixture was incubated for $30 \mathrm{~min}$ at $70^{\circ} \mathrm{C}$.

\subsubsection{Ligation}

During a ligation reaction, the T4 DNA ligase catalyzes the formation of a phosphodiester bond between the $3^{\prime}$-hydroxy- and 5'- phosphate group of linearized DNA, resulting in the generation of recombinant DNA molecules.

\section{Ligation with pGEM®-T Easy}

After adding A-overhangs to the PCR product the A-tailing mixture was cloned into the pGEM®T Easy vector (Promega, Wisconsin, USA). The A-overhangs of the PCR product anneal to the 3 '-thymidine overhangs of the T-cloning PGEM®-T Easy vector. The following reaction mixture with a total volume of $10 \mu \mathrm{l}$ was used:

$$
\begin{aligned}
& 1 \mu \mathrm{l} \text { pGEM®T-Easy vector } \\
& 1 \mu \mathrm{l} \mathrm{T4} \text { DNA ligase } \\
& 5 \mu \mathrm{l} 2 \mathrm{x} \text { rapid ligation buffer } \\
& 1 \mu \mathrm{l} \mathrm{PCR} \text { product (insert) } \\
& 2 \mu \mathrm{l} \mathrm{H}_{2} \mathrm{O}
\end{aligned}
$$

The reaction was kept for $30 \mathrm{~min}$ at RT before it was transferred for overnight incubation to $4^{\circ} \mathrm{C}$. The following day continued with the transformation of competent DH5a-cells.

\section{Ligation with pIRES2-EGFP or pEBTetD}

The pGEM®-T Easy-Ccnd2 plasmid was digested overnight with the corresponding enzymes. After the digestion was run on an agarose gel and the cyclin D2-insert DNA-band was excised 
and purified it was ligated into the corresponding vector pIRES2-EGFP or pEBTetD in multiple insert-DNA to vector-DNA ratios, ranging from 1:1, $2: 1$ to $3: 1$.

\author{
$1 \mu \mathrm{l}$ vector (digested) \\ 1-3 $\mu$ I PCR product (insert) \\ $5 \mu \mathrm{l} 2 \mathrm{x}$ rapid ligation buffer \\ ad. $\mathrm{H}_{2} \mathrm{O}$ to final volume of $9 \mu \mathrm{l}$
}

The reaction mixture was incubated for $5 \mathrm{~min}$ at $65^{\circ} \mathrm{C}$ and then for $5 \mathrm{~min}$ on ice. Finally, $1 \mu \mathrm{T} 4$ DNA ligase was added. The reaction was kept for $30 \mathrm{~min}$ at RT before it was transferred for overnight incubation to $4^{\circ} \mathrm{C}$ or to $16^{\circ} \mathrm{C}$ thermocycler.

\title{
2.12.6 Transformation
}

A $50 \mu \mathrm{l}$ aliquot of competent bacteria (E.coli DH5a) was thawed for 5-10 min on ice. 2-7 $\mu \mathrm{l}$ of the ligation reaction were added and gently mixed with the bacteria. After incubation for 30 min on ice a $45 \mathrm{sec}$. heat shock was performed at $42^{\circ} \mathrm{C}$. The cells cooled down again for 5 min on ice before $200 \mu$ LB medium were added and mixed by incubation on a thermomixer for 1 hour at $37^{\circ} \mathrm{C}$ and $1000 \mathrm{rpm}$. Finally, the transformed bacteria were plated out on LB agar plates containing the appropriate antibiotic. The plates were incubated overnight at $37^{\circ} \mathrm{C}$ (Hanahan 1983). Bacteria transformed with the $P G E M ®-T$ Easy plasmid had to be plated on $L B$ agar plates not only containing the appropriate antibiotic but also X-Gal for blue and white screening (10 $\mu \mathrm{l}$ $\mathrm{X}$-Gal in $100 \mu \mathrm{H} 2 \mathrm{O}$ ). A blue colored colony indicates that the lacZ gene in pGEM®-T Easy is intact (no insert) whereas a white colony indicates that the gene is disrupted (insert present, Langley et al. 1975, Vieira and Messing 1982).

\subsubsection{Generation of bacterial glycerol stocks}

$10 \%$ sterile glycerol was added to the bacterial suspension, mixed well by vortexing and stored at $-80^{\circ} \mathrm{C}$ for future purposes.

\subsubsection{Minipreparation of plasmid DNA}

Small amounts of plasmid DNA were prepared for testing by restriction endonuclease digestion whether the plasmid contains the desired insert or not. Therefore, $1 \mathrm{ml} \mathrm{LB}$ medium, together with 
$1 \mu \mathrm{l}$ of the corresponding antibiotic, was inoculated with a single clone from the agar plate and incubated overnight on a thermomixer at $37^{\circ} \mathrm{C}$ and $600 \mathrm{rpm}$. The following day the bacterial overnight-culture was centrifuged at $5000 \times \mathrm{g}$. The pellet was resuspended in $250 \mu \mathrm{P} 1$ buffer and $250 \mu \mathrm{l}$ of P2 buffer (modified alkaline lysis) were added afterwards. The suspension was incubated for $5 \mathrm{~min}$ at RT before $250 \mu \mathrm{l}$ P3 were added (for neutralization). The sample was centrifuged for $10 \mathrm{~min}$ at $4^{\circ} \mathrm{C}$ and $13000 \mathrm{rpm}$. The resulting supernatant was transferred to a 1.5 $\mathrm{ml}$ reaction tube and $500 \mu \mathrm{l}$ isopropanol were added. The solution was well mixed and precipitated by centrifugation for $30 \mathrm{~min}$ at $4^{\circ} \mathrm{C}$ and $13000 \mathrm{rpm}$. Thereafter the DNA pellet was washed with $200 \mu \mathrm{l}$ of $70 \%$ ethanol and centrifugation for $5 \mathrm{~min}$ at $13000 \mathrm{rpm}$. The supernatant was discarded and the plasmid DNA pellet was dried and resolved in $20 \mu \mathrm{ddH}_{2} \mathrm{O}$.

\subsubsection{Midipreparation of plasmid DNA}

Large amounts of plasmid DNA were needed for restriction analysis, subcloning, sequencing or transfection of cell lines. To generate these large amounts of plasmid DNA, $50 \mathrm{ml}$ LB medium together with $50 \mu \mathrm{l}$ of the corresponding antibiotic were inoculated with either $50 \mu$ bacterial overnight-culture (taken before Minipreparation and stored at $4^{\circ} \mathrm{C}$ ) or with a swap of a glycerol stock. The solution was incubated overnight on a shaker at $37^{\circ} \mathrm{C}$. Plasmid DNA of the resulting bacterial overnight-culture was isolated by using the PureLink ${ }^{\mathrm{TM}}$ HiPure Plasmid Midiprep Kit (Life Technologies, Darmstadt, Germany) which uses affinity chromatography columns to purify the DNA. The purification was performed according to the manufactures instructions.

\subsubsection{Cleavage of DNA with restriction endonucleases}

Enzymatic cleavage of DNA by restriction enzymes was prepared in volumes ranging from $10 \mu \mathrm{l}$ for test digestions, up to $100 \mu$ for isolation of DNA fragments from agarose gels. Per $\mu g$ DNA 1 $U$ of the respective restriction enzyme was used together with the corresponding buffer. The reaction was incubated at $37^{\circ} \mathrm{C}$ for $30 \mathrm{~min}$ for test digestions or overnight for gel extractions. When two restriction enzymes had to be used, either a buffer was chosen that ensured sufficient activity of the endonucleases or a sequential restriction was performed.

\subsubsection{Isolation of DNA fragments from agarose gels}

DNA fragments resulting from cleavage with restriction enzymes were separated by gel electrophoresis on an agarose gel. The desired fragment was excised from the gel using a sterile 
scalpel and dissolved at $55^{\circ} \mathrm{C}$ in the appropriate amount of Binding Buffer NTI. Binding buffer $\mathrm{NTI}$ is supplied within the NucleoSpin ${ }^{\mathrm{TM}}$ Extract II Kit (Machery \& Nagel, Düren, Germany), which was used for further isolation of the DNA from the gel slice according to the manufacturer's instructions. The DNA was eluted in $\mathrm{ddH}_{2} \mathrm{O}$.

\subsection{Agarose gel electrophoresis of DNA}

During agarose gel electrophoresis an electric field is used to separate charged macromolecules, such as nucleic acids, according to their size. Small fragments will move further through the gel than larger fragments. The resolution of the separation can be influenced by the concentration of the gel and the charge to mass ratio of the corresponding macromolecule. Gels with an agarose concentration of $0.5-2.0 \%(\mathrm{w} / \mathrm{v})$ were prepared, depending on the size of the fragments to be separated. The agarose was resolved in the appropriate amount of $1 x$ Turbo buffer by boiling. DNA Stain G (Serva $\mathrm{GmbH}$, Heidelberg, Germany), which intercalates with DNA, was added to visualize the DNA. The agarose gel was poured into a horizontal gel chamber to cool out. When the gel was completely hardened it was loaded with the samples and a length standard in order to be able to determine the length of the separated DNA fragments. The following length standards were used: $1 \mathrm{~Kb}$ Plus DNA-ladder (Life Technologies, Darmstadt, Germany) or MassRuler® (MBI Fermentas, St. Leon-Rot, Germany). The gel electrophoresis was performed in $1 \times$ Turbo buffer at a constant voltage of $200 \mathrm{~V}$ and $200 \mathrm{~mA}$. For documentation a picture of the gel was taken on an UV table/transilluminator.

\subsection{Polymerase-Chain-reaction (PCR)}

The polymerase chain reaction is a standard method to amplify specific DNA fragments (Saiki et al. 1985, Mullis and Faloona 1987). Thereby double stranded DNA is denatured at high temperatures, which allows short oligonucleotides (primers) to bind to the single stranded DNA (annealing). Once bound, these oligonucleotides will be extended by the polymerase (elongation). The periodic repetition of the denaturation, annealing and elongation steps results in an exponential increase in DNA fragments. Whether the PCR was successful can be checked by agarose gel electrophoresis. 


\subsubsection{Standard PCR}

The standard PCR was used to control the efficiency of the cDNA synthesis after reverse transcription PCR (Section 2.14.5). Therefore, specific primers for the housekeeping genes glyceraldehyde 3-phosphate dehydrogenase (GAPDH, for human samples) or hypoxanthineguanine phosphoribosyltransferase (Hprt, for mouse samples) were used. The following reaction was used:

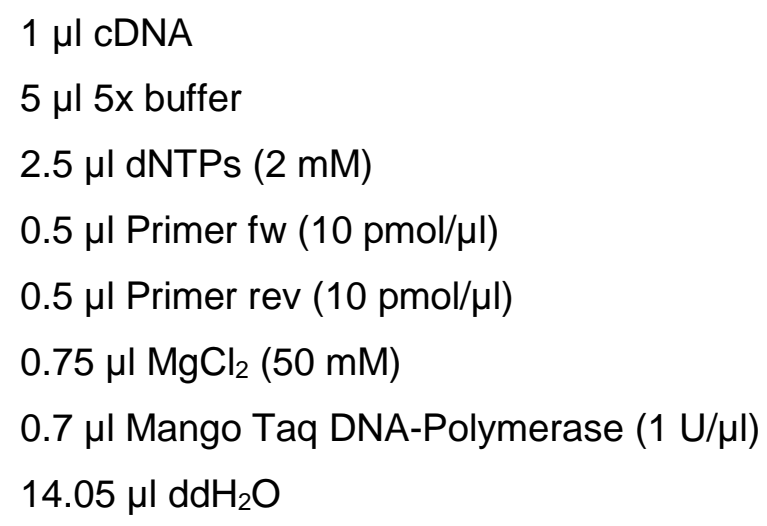

The reaction mixture was afterwards incubated on a Thermocycler (2720 Thermal Cycler, Applied Biosystems, California, USA) using the following program:

$\left.\begin{array}{lll}\text { Initial denaturation } & 94^{\circ} \mathrm{C} & 5 \mathrm{~min} \\ \text { Denaturation } & 94^{\circ} \mathrm{C} & 30 \mathrm{sec} \\ \text { Annealing } & 60^{\circ} \mathrm{C} & 30 \mathrm{sec} \\ \text { Elongation } & 72^{\circ} \mathrm{C} & 30 \mathrm{sec}\end{array}\right\} 30 \mathrm{x}$

\subsubsection{Amplification of DNA fragments for molecular cloning/ Touchdown PCR}

A specific pair of primers, defining the DNA-region of interest designated for cloning into the pGEM®-T Easy vector (Promega, Wisconsin, USA), was designed and purchased. DNA fragments selected for eventual cloning were amplified with the Phusion ${ }^{\mathrm{TM}}$ High-Fidelity DNA polymerase (Finnzymes, Finnland). This polymerase encompasses not only the $5^{\prime} \rightarrow 3^{\prime}$ DNA polymerase activity but also a $3^{\prime} \rightarrow 5^{\prime}$ exonuclease (proofreading) activity. The following PCR reaction mixture was used: 


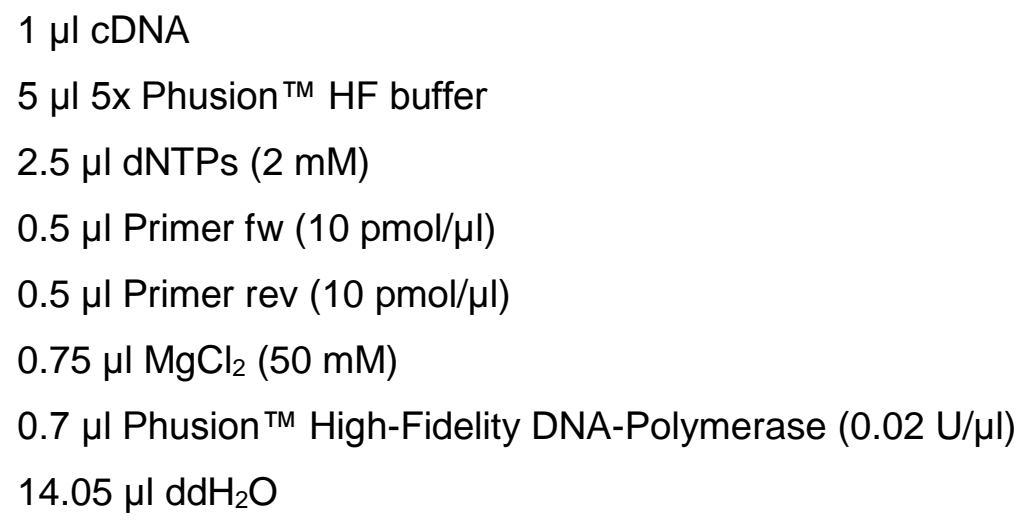

To increase specificity, sensitivity and yield, a touchdown PCR program was applied. The initial annealing temperature will be above the projected melting temperature $\left(T_{\mathrm{m}}\right)$ of the used primers, then it progressively transitions to a lower, more permissive annealing temperature over the course of successive cycles (Korbie and Mattick 2008). The primer will anneal at the highest temperature which is least-permissive of nonspecific binding. The generated fragments will be further amplified during subsequent rounds at lower temperatures and will out-compete the nonspecific sequences to which the primers may bind at those lower temperatures (Don et al. 1991, Hecker and Roux 1996).

The reaction was carried out in the Primus 25 advanced Thermocycler from Peqlab (Erlangen, Germany). The following Touchdown PCR program was used:

\begin{tabular}{|c|c|c|c|c|}
\hline Initial denaturation & $98^{\circ} \mathrm{C}$ & $30 \mathrm{sec}$ & & \\
\hline Denaturation & $98^{\circ} \mathrm{C}$ & $10 \mathrm{sec}$ & & \\
\hline Annealing & $65^{\circ} \mathrm{C}$ & $30 \mathrm{sec}$ & $-1^{\circ} \mathrm{C}$ & $10 x$ \\
\hline Elongation & $72^{\circ} \mathrm{C}$ & $3 \mathrm{~min}$ & & \\
\hline Denaturation & $98^{\circ} \mathrm{C}$ & $10 \mathrm{sec}$ & & \\
\hline Annealing & $58^{\circ} \mathrm{C}$ & $30 \mathrm{sec}$ & & \\
\hline Elongation & $72^{\circ} \mathrm{C}$ & $3 \min$ & & \\
\hline
\end{tabular}

\subsubsection{Colony PCR}

Colony PCR is a method used to examine many different clones in a short period of time. In this study, it was conducted to identify clones from an agar plate after ligation and transformation procedure, which have integrated the desired PCR product. The following reaction was used: 


$$
\begin{aligned}
& 1 \mu \mathrm{l} \text { Primer fw }(10 \mathrm{pmol} / \mu \mathrm{l}) \\
& 1 \mu \mathrm{l} \text { Primer rev }(10 \mathrm{pmol} / \mu \mathrm{l}) \\
& 2.5 \mu \mathrm{dNTPs}(2 \mathrm{mM}) \\
& 5 \mu \mathrm{l} 5 \mathrm{x} \text { buffer } \\
& 0.75 \mu \mathrm{l} \mathrm{MgCl}_{2} \\
& 0.5 \mu \text { l Mango Taq-Polymerase } \\
& 14.25 \mu \mathrm{ddH}_{2} \mathrm{O}
\end{aligned}
$$

The reaction mixture was inoculated with a single clone picked from the agar plate with a pipette tip. As a backup, the pipette tip with the clone was also smeared on a fresh and sterile agar plate containing the appropriate antibiotic. The mixture was incubated on a thermocycler (2720 Thermal Cycler, Applied Biosystems, California, USA) using the following program:

$\left.\begin{array}{lll}\text { Initial denaturation } & 95^{\circ} \mathrm{C} & 5 \mathrm{~min} \\ \text { Denaturation } & 95^{\circ} \mathrm{C} & 30 \mathrm{sec} \\ \text { Annealing } & 60^{\circ} \mathrm{C} & 30 \mathrm{sec} \\ \text { Elongation } & 72^{\circ} \mathrm{C} & 50 \mathrm{sec}\end{array}\right\} 30 \mathrm{x}$

\subsubsection{Genotyping PCR}

For the mouse genotyping PCR, DNA had to be isolated first from the tail biopsy. Therefore, the DirectPCR $®$ Lysis Reagent Tail from Peqlab (Erlangen, Germany) was used. $200 \mu$ of the lysis reagent were added to the biopsy as well as $6 \mu$ l Proteinase $\mathrm{K}(10 \mathrm{mg} / \mathrm{ml})$. For lysis, the reaction was incubated overnight at $55^{\circ} \mathrm{C}$ and $600 \mathrm{rpm}$ on a thermomixer. The following day the reaction was incubated for $45 \mathrm{~min}$ at $55^{\circ} \mathrm{C}$ for heat inactivation and then transferred on ice and used for the following PCR reaction: 
$1 \mu \mathrm{DNA}$

$5 \mu \mathrm{l} 5 \mathrm{x}$ buffer

$2.5 \mu \mathrm{dNTPs}(2 \mathrm{mM})$

$0.5 \mu \mathrm{l}$ Primer fw $(10 \mathrm{pmol} / \mu \mathrm{l})$

$0.5 \mu \mathrm{l}$ Primer rev $(10 \mathrm{pmol} / \mu \mathrm{l})$

$0.75 \mu \mathrm{MgCl}_{2}(50 \mathrm{mM})$

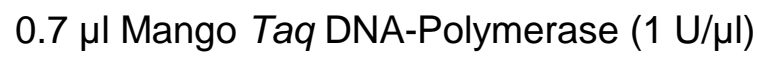

$14.05 \mu \mathrm{ldd} \mathrm{H}_{2} \mathrm{O}$

The reaction mixture was afterwards incubated on a Thermocycler (2720 Thermal Cycler, Applied Biosystems, California, USA) using the following program:

Initial denaturation

Denaturation

Annealing

Elongation

Final elongation

Storage

$\left.\begin{array}{ll}95^{\circ} \mathrm{C} & 5 \mathrm{~min} \\ 95^{\circ} \mathrm{C} & 30 \mathrm{sec} \\ 61^{\circ} \mathrm{C} & 1 \mathrm{~min} \\ 72^{\circ} \mathrm{C} & 1 \mathrm{~min} \\ 72^{\circ} \mathrm{C} & 10 \mathrm{~min} \\ 8^{\circ} \mathrm{C} & \infty\end{array}\right\} 35 \mathrm{x}$

\subsubsection{Reverse Transcription}

The reverse transcriptase used in the reverse transcription (RT) PCR transcribes RNA into cDNA. The principle of reverse transcription is, that the added oligo(dT)-primers anneal to the poly(A)-sequences of mRNAs. Corresponding to the standard PCR, the reverse transcriptase can elongate the strand to synthesize cDNA. For the generation of cDNA from total RNA the reverse transcriptase SuperScript II from Life Technologies (Darmstadt, Germany) was used. Eventually, the cDNA was subject to quantitative real-time PCR analysis. The reaction was performed in three following steps:

1. Step:

$$
1-5 \mu g \text { RNA }
$$

Added to a $6 \mu$ volume with $\mathrm{ddH}_{2} \mathrm{O}$

$+0.5 \mu$ l oligo(dT)-primer $(0.5 \mu \mathrm{g} / \mu \mathrm{l})$

$+0.5 \mu \mathrm{d}$ dNTPs (10 mM)

Incubation: $10 \mathrm{~min}$ at $65^{\circ} \mathrm{C}$ 
2. Step: $\quad+2 \mu$ l $5 x$ First Strand Buffer

$+1 \mu \mathrm{ITTT}(0.1 \mathrm{M})$

Incubation: 2 min at $42^{\circ} \mathrm{C}$

3. Step: $\quad+0.25 \mu$ l SuperScript II

$+0.75 \mu \mathrm{ddd} \mathrm{H}_{2} \mathrm{O}$

Incubation for reverse transcription: $50 \mathrm{~min}$ at $42^{\circ} \mathrm{C}$

Heat-inactivation: $15 \mathrm{~min}$ at $75^{\circ} \mathrm{C}$

Storage: $8^{\circ} \mathrm{C} \infty$

The cDNA was diluted 1:10 with $\mathrm{ddH}_{2} \mathrm{O}$. Before the generated cDNA was subjected to quantitative real-time PCR analyses, the quality of the reverse transcription was analyzed by standard PCR (Section 2.14.1) using specific primers for the housekeeping genes GAPDH or HPRT, depending on the source of the RNA.

\subsubsection{Quantitative real-time PCR}

Basically, the quantitative real-time PCR relies on the principal of the conventional PCR, but additionally, it enables the quantification of the PCR product during the PCR. This is possible due to the measurement of a fluorescent dye which binds to double stranded DNA and thereby increases its fluorescent signal (Pfaffl 2001, Ponchel et al. 2003). Thus, the increase in the measured fluorescent signal correlates to the amount of generated DNA. The fluorescent dye used in this study was PCR Mastermix Platinum® SYBR® Green qPCR SuperMix-UDG with Rox (Life Technologies, Darmstadt, Germany). Double stranded DNA bound with SYBR® Green can be excited using light of $480 \mathrm{~nm}$ wavelength resulting in an emission spectrum with a maximum at $520 \mathrm{~nm}$. The Rox reference dye serves for normalization of non-PCR-related fluctuations in fluorescence. Using the ABI Prism 7900T Sequence Detection System the increase in the fluorescent intensity was measured after every PCR cycle and hence a graph was generated. During the exponential phase of the PCR reaction, in which the conditions are optimal by means of optimal polymerase activity and sufficient amount of reaction materials (primers, $\mathrm{MgCl}_{2}$ ), the threshold value is determined. This value defines the PCR cycle with the optimal conditions and it is used for further quantification calculations (Ct value). The generated data was evaluated with the Sequence Detection System software (SDS Version 2.1, PE Applied Biosystems). Usually, the used cDNA was diluted 1:20 with $\mathrm{dd}_{2} \mathrm{O}$ prior to the quantitative realtime PCR. The reaction for one well of a 396- well plate consisted of the following components: 


\section{$2.5 \mu \mathrm{l} \mathrm{cDNA}$ \\ $2.5 \mu \mathrm{l}$ Primer (fw + rev $100 \mathrm{pmol} / \mu \mathrm{l})$ \\ $5.0 \mu \mathrm{l} \mathrm{SYBR}{ }^{\circledR}$ Green}

The relative expression for one sample was measured in duplicates of which the mean value was used for the final calculations. The following program was used on the ABI Prism 7900T Sequence Detection system:

$\begin{array}{lll} & 50^{\circ} \mathrm{C} & 2 \mathrm{~min} \\ \text { Taq activation: } & 95^{\circ} \mathrm{C} & 3 \mathrm{~min} \\ \text { Denaturation: } & 94^{\circ} \mathrm{C} & 15 \mathrm{sec} \\ \text { Annealing: } & 60^{\circ} \mathrm{C} & 30 \mathrm{sec} \\ \text { Elongation: } & 72^{\circ} \mathrm{C} & 30 \mathrm{sec} \\ & 95^{\circ} \mathrm{C} & 15 \mathrm{sec} \\ & 60^{\circ} \mathrm{C} & 15 \mathrm{sec} \\ & 60^{\circ} \mathrm{C}-95^{\circ} \mathrm{C} & 2^{\circ} \mathrm{C} / \mathrm{min}\end{array} 40 \mathrm{x}$

After completion of the run, the data was evaluated with the SDS program and transferred to MS Excel (Microsoft) for further calculations. The relative expression was determined by $\Delta \Delta$-Ctmethod for which the following formulae were used:

$$
\begin{aligned}
& \Delta \mathrm{Ct}=\mathrm{Ct} \text { (Gene of interest) }-\mathrm{Ct} \text { (housekeeping gene) } \\
& \Delta \Delta \mathrm{Ct}=\Delta \mathrm{Ct} \text { (Control) }-\Delta \mathrm{Ct} \text { (sample of interest) } \\
& \text { Relative expression }=2^{\Delta \Delta \mathrm{Ct}}
\end{aligned}
$$

The mRNA expression of two housekeeping genes, including TBP (TATA box binding protein), PBGD (porphobilinogen deaminase), LDHA (Lactate dehydrogenase A) or HPRT (Hypoxanthine-guanine phosphoribosyltransferase) for human samples was used as a reference. For murine samples Hprt (hypoxanthine-guaninephosphoribosyltransferase) und Tbp (TATA box binding protein) were used for normalization.

\subsubsection{Sequence analysis}

The standard, non-radioactive sequence analysis according to Sanger is based on the principle of chain termination (Sanger and Coulson 1975). During the sequence reaction not only deoxy- 
nucleosides, but also dideoxynucleotides that have been labeled with different fluorescent dyes, will be incorporated into the DNA during the elongation step. Since the dideoxynucleotides do not possess a 3'- hydroxyl group no further nucleotides can be added by the polymerase resulting in a chain termination. Due to the detected dye the nucleotide can be determined and due to the length of the fragments to position of the nucleotides within the DNA strand could be determined. The used reaction consisted of the following components in a total volume of $10 \mu \mathrm{l}$ :

$$
\begin{aligned}
& 1 \mu \mathrm{l} \text { DNA } \\
& 2 \mu \mathrm{l} 5 \mathrm{x} \text { Buffer } \\
& 1 \mu \mathrm{l} \text { BigDye® } \\
& 1 \mu \mathrm{l} \text { sequence-specific primer, either the fw or rev }(10 \mathrm{pmol} / \mu \mathrm{l}) \\
& 5 \mu \mathrm{l} \mathrm{H}_{2} \mathrm{O}
\end{aligned}
$$

The reaction was incubated on a Thermocycler (2720 Thermal Cycler, Applied Biosystems, California, USA) using the following program:

Initial denaturation

Denaturation

Annealing

Elongation

Storage

$\left.\begin{array}{ll}95^{\circ} \mathrm{C} & 1 \mathrm{~min} \\ 95^{\circ} \mathrm{C} & 30 \mathrm{sec} \\ 60^{\circ} \mathrm{C} & 2.5 \mathrm{~min} \\ 60^{\circ} \mathrm{C} & 5 \mathrm{~min}\end{array}\right\} 30 \mathrm{x}$

$8^{\circ} \mathrm{C} \quad \infty$

When the reaction was completed, $10 \mu \mathrm{lddH_{2 }} \mathrm{O}$ were added to the sample. Gel electrophoresis by specific capillaries, which detect the fluorescent signals of the dideoxynucleotides, was performed in the automatic sequencer 3500XL (Applied Biosystems, Life Technologies, Darmstadt, Germany).

\subsection{Protein chemical techniques}

\subsubsection{Isolation of total protein from cell cultures}

For isolation of total protein from cell cultures the medium was first removed and then the cells were washed with ice cold PBS. Depending on the confluency of the cells and the used culture flask/plate, the appropriate amount of protein lysis buffer (modified RIPA) was added to the 
adherent cells; for example $150 \mu$ lysis buffer were used for cells with a $90 \%$ confluency in a 6 well plate. The cells were incubated for $5 \mathrm{~min}$ on ice before they were scraped off the bottom with either a pipette tip or a cell scraper and transferred to a reaction tube. To pellet the cell debris the cells were centrifuged at $13000 \mathrm{rpm}$ at $4^{\circ} \mathrm{C}$ for $10 \mathrm{~min}$. The supernatant, which consists of the isolated protein, was transferred to a new reaction tube and the protein concentration was measured. For short-term storage the protein was kept at $-20^{\circ} \mathrm{C}$, for longterm storage at $-80^{\circ} \mathrm{C}$.

\subsubsection{Isolation of protein from mouse tissue}

Proteins from mouse prostate or prostate tumor tissue was obtained via homogenization of the tissue using the TissueLyser LT (Quiagen, Hilden, Germany). A small sample of the tissue was transferred to a $2 \mathrm{ml}$ reaction tube. A stainless steel bead ( $\varnothing 5 \mathrm{~mm}$, Quiagen) was added as well as $500 \mu \mathrm{l}$ modified RIPA lysis buffer. The tissue was homogenized for $5 \mathrm{~min}$ at $50 \mathrm{1} / \mathrm{s}$ oscillation in the TissueLyser LT. Thereafter the cups were kept for 5 min on ice before centrifuging them for $10 \mathrm{~min}$ at $13000 \mathrm{rpm}$ and $4^{\circ} \mathrm{C}$. The supernatant was transferred to a new $1.5 \mathrm{ml}$ reaction tube and the protein concentration was measured. For short-term storage the protein was kept at $20^{\circ} \mathrm{C}$ and for long-term storage at $-80^{\circ} \mathrm{C}$.

\subsubsection{Determination of protein concentration}

The protein concentration was measured via Bradford protein assay (Bradford 1976). Roti®Nanoquant (Carl Roth $\mathrm{GmbH}$, Karlsruhe, Germany) was used, which consists of the Coomassie Brilliant blue dye. In acidic solutions, this dye binds unspecific to cationic and hydrophobic side chains of proteins. Once bound, the absorption maximum of the dye is shifted from $495 \mathrm{~nm}$ to $595 \mathrm{~nm}$ since the binding reaction stabilizes the dye in its non-protonated and anionic form. A series of Roth Albumin Fraction $V$ dilution was used for calibration according to the manufacturer's recommendation. The protein concentration was determined by extrapolating to this standard curve.

$5 x$ Rotiß- Nanoquant dye was diluted with $\mathrm{ddH}_{2} \mathrm{O}$ to a final $1 \mathrm{x}$ concentration and stored at $4^{\circ} \mathrm{C}$. Protein samples were diluted 1:100 with $\mathrm{ddH}_{2} \mathrm{O}$. $50 \mu \mathrm{l}$ of the sample were added to a 96-well plate in triplicates. $\mathrm{ddH}_{2} \mathrm{O}$ was used as a control. $200 \mu \mathrm{l}$ of $1 \mathrm{x}$ Roti®- Nanoquant were added to samples and incubated for $5 \mathrm{~min}$ at RT. The protein concentration was measured using the SynergyMx plate reader spectrophotometer (BioTek, Friedrichshall, Germany) and calculated by inherent Gene5 software. 


\subsubsection{Sodium Dodecyl Sulfate Polyacrylamide gel electrophoresis (SDS-PAGE)}

SDS-PAGE gel electrophoresis (Western blot) is a useful biochemical method to separate proteins of a sample according to their molecular weight. The NuPage® Pre-Cast Gel System from Life Technologies (Darmstadt, Germany) was used which is based on the SDS-PAGE gel chemistry by Laemmli (Laemmli 1970). This polyacrylamide gel system, designed for high performance gel electrophoresis, consists of pre-cast gels and buffers with an operating $\mathrm{pH}$ of 7.0, which increases stability in both proteins and gels. The samples consisted of 20-30 $\mathrm{gg}$ protein, 0.25 vol sample buffer (LDS Sample Buffer (4x), NuPAGE®), Life Technologies, Darmstadt, Germany) and $10 \% 1 \mathrm{M} \mathrm{DTT}$ (reducing agent). The samples were denatured at $70^{\circ} \mathrm{C}$ for $10 \mathrm{~min}$ and afterwards kept on ice for $5 \mathrm{~min}$. Before the samples were loaded onto the gradient gel (NuPAGE® 4-12\% Bis-Tris Gel, Life Technologies, Darmstadt, Germany), they were centrifuged briefly. $7 \mu$ of the pre-stained molecular weight standard (See Blue®) Plus2, Life Technologies, Darmstadt, Germany) were also loaded onto the gel to determine the size of the separated proteins. Gel electrophoresis was performed at $160 \mathrm{~V}$ and $160 \mathrm{~mA}$ in 1x MES buffer (Life Technologies, Darmstadt, Germany). Depending on the molecular weight of the protein of interest the gel was run for 1-3 hours.

\subsubsection{Transfer of proteins onto a PVDF membrane}

After SDS-PAGE gel electrophoresis the proteins were transferred onto a PVDF membrane (GE Healthcare, Freiburg, Germany) using the semi-dry blotting procedure. Thereby, the PVDF membrane $(7 \mathrm{~cm} \times 8 \mathrm{~cm}$ or $7 \mathrm{~cm} \times 13 \mathrm{~cm}$ ) was first activated for $10 \mathrm{sec}$ in $100 \%$ methanol before it was equilibrated for 10 min in transfer buffer Ila. Six sheets of Whatman GB003 filter paper (Schleicher \& Schull, Dassel, Germany) having the same size as the gel and the PVDF membrane, were also soaked in transfer buffer lla. The electro-blotter (Biometra, Göttingen, Germany) was loaded in a sandwich system in the following order:

Bottom of the blotter, Anode (+): Three sheets of filter paper PVDF membrane

Gel

Lid of the blotter, Cathode (-): $\quad$ Three sheets of filter paper

Before the blotter was closed, remaining air bubbles were removed from the sandwich system. The transfer was carried out at $25 \mathrm{~V}$ and $220 \mathrm{~mA}$ for $30 \mathrm{~min}$ to 1 hour, depending on the molecular weight of the proteins. 


\subsubsection{Incubation of protein-bound membranes with antibodies}

The PVDF membrane was incubated in the western blot blocking buffer for 1 hour at RT to block unspecific binding sites. Thereafter it was thoroughly washed with western blot washing buffer. The membrane was incubated with the primary antibody overnight at the recommended dilution in TBS-T at $4{ }^{\circ} \mathrm{C}$. The next day, unbound antibody was removed by washing twice for $10 \mathrm{~min}$ with the western blot washing buffer. The membrane was then incubated for at least 2 hours with the secondary alkaline phosphatase conjugated antibody, diluted accordingly to the manufacturer's recommendation in western blot blocking buffer. It was washed three times for $15 \mathrm{~min}$ in western blot washing buffer and $5 \mathrm{~min}$ in TBS-T to remove the remaining milk. ECL Prime Detection solution (GE Healthcare, Freiburg, Germany) was used to detect chemiluminescent signals. Therefore, the membrane was placed on a plastic film and the detection solution was pipetted onto the membrane according to the manufacturer's instruction and incubated for $5 \mathrm{~min}$. The signals were captured using the western blot detection system (FlourChem ${ }^{\circledR}$ Q Alpha Innotech, Logan, USA) and evaluated using the inherent AlphaView Software for FluorChem ${ }^{\circledR}$ systems (FlourChem® ${ }^{\circledR}$ Alpha Innotech, Logan, USA).

\subsection{Cell biological methods}

\subsubsection{Cell culture of eukaryotic cells}

Cells were cultured in their appropriate medium (see section 2.8.3) in surface-treated cell culture flasks (Sarstedt, Nümbrecht, Germany) at $37^{\circ} \mathrm{C}$ in a humidified incubator with $5 \% \mathrm{CO}_{2}$. Depending on the proliferation rate and confluency of the cells, they were splitted once or twice per week. For the splitting process the cells were first washed with DBPS (PAN Biotech GmbH, Aidenbach, Germany) and then incubated for a few minutes at $37^{\circ} \mathrm{C}$ in a minimal amount of Trypsin/EDTA (PAN Biotech GmbH, Aidenbach, Germany) for detachment from the culture flask. Using an inverted microscope it was controlled whether all cells were detached. If this was the case, the trypsin reaction was stopped by adding growth medium. Depending on proliferation rate of the cells they were diluted either $1: 5$ or 1:10 with fresh culture medium.

\subsubsection{Cryo-preservation and revitalization of eukaryotic cells}

For cryo-preservation, the cells were grown to $80 \%$ confluency and then trypsinized as described in 2.16.1. When all the cells had detached from the bottom of the cell culture flask the 
tryspin reaction was stopped with medium. The cells were transferred to a tube and centrifuged at $200 \times \mathrm{g}$ for $5 \mathrm{~min}$ to form a cell pellet and remove the remaining trypsin. The pellet was resuspended in the appropriate amount of culture medium and then diluted 1:1 with cryo-medium (see 2.8.3). Overnight, the cells were kept in Mr. Frosty (Thermo Scientific, Langenselbold, Germany) in a $-80^{\circ} \mathrm{C}$ freezer where the cells were slowly cooled $\left(1^{\circ} \mathrm{C} / \mathrm{min}\right)$. The following day, the cells were transferred to liquid nitrogen for long-term storage. When cells were revitalized, they were quickly thawed and transferred to a tube containing $5 \mathrm{ml}$ of the appropriate medium. The cells were centrifuged at $200 \times \mathrm{g}$ for $5 \mathrm{~min}$, the supernatant was removed and the cell pellet was resuspended in pre-warmed culture medium. The cells were then transferred to a cell culture flask and kept in the incubator. The next day the medium had to be changed in order to fully remove remaining DMSO.

\subsubsection{Test for Mycoplasma contamination}

To detect a possible contamination by Mycoplasma, a routinely test was conducted every month. Therefore, the MycoAlert® Mycoplasma Detection Kit (Lonza, Cologne, Germany) was used according to the manufacturer's instructions, except that only half of the recommended amounts were used. The principle of this test is the measurement of the activity of mycoplasma-specific enzymes before and after application of specific substrates.

\subsubsection{Transfection of eukaryotic cells}

\subsubsection{Transfection of plasmids into eukaryotic cells}

Eukaryotic cells were transfected with plasmids in order to induce an overexpression of fusion proteins (pIRES2-EGFP-CCND2, pEBTetD-CCND2) or to induce a downregulation of the fusion protein by shRNA (pSingle-tTs-Ccnd2-shRNA). Two different transfection reagents were used: Lipofectamine ${ }^{\circledR}$ Transfection Reagent (Thermo Fisher Scientific, Langenselbold, Germany) or Metafectene® Pro (Biontex Laboratories $\mathrm{GmbH}$, Munich, Germany). Both transfection reagents contain lipids which form vesicles with a bilayer sheet, so called liposomes, in aqueous solution. The liposomes can form nucleic acid lipid complexes with nucleic acids. These complexes can actively be taken up by the eukaryotic cells by a process called endocytosis. The nucleic acid can enter the nucleus only if the nuclear membrane dissolves during mitosis. Therefore, the division rate of cells is critical for DNA transfection and must be as high as possible for efficient transfection. The cells were plated in a sufficient number into 6-well cell culture plates or T-25 
cell culture flasks so that the following day a confluency of $70-90 \%$ was reached. For the transfection, plasmid DNA was mixed with the transfection reagent according to the manufacturer's instructions. The mix was incubated for 20 - $30 \mathrm{~min}$ and then applied dropwise to the cells in normal growth medium, which were prior washed with DPBS. The cells were incubated overnight at normal culture conditions. The following day, the transfection reagentcontaining medium was replaced by fresh medium.

\subsection{Generation of single-cell clones/populations}

Cells transfected with the pEBTetD-CCND2 plasmid were treated from the next day on with puromycin for clonal selection of successfully transfected cells (see Chapter 2.8.3.). The surviving cells were further cultured until the amount of cells was sufficient to test by western blot analysis if the cells indeed exhibit a cyclin D2 overexpression. Total protein was isolated after the cells were treated with doxycycline in different concentrations $(0.01 \mu \mathrm{g} / \mathrm{ml}-1 \mu \mathrm{g} / \mathrm{ml})$ for 48 or 72 hours to induce cyclin D2 overexpression. Successfully generated cells with a doxycycline-inducible cyclin D2 overexpression were preserved by cryo-preservation and subject to proliferation studies.

Cells transfected with the plasmid pIRES2-EGFP-CCND2 or pSingle-tTs-Ccnd2-shRNA underwent a serial dilution with normal growth medium, ranging from 1:10 - 1:80, in order to generate single-cell clones. This was done two days after the transfection. The diluted cells were plated in cell culture petri dishes and cultured under normal cell culture conditions. From the next day on the cells received Geneticin disulfate (G418)-solution with their normal growth medium in order to select positively transfected cells. Approximately three weeks after the serial dilution of the cells, single cells had formed clones which were picked with a pipette, transferred to a 96well plate and incubated at normal culture conditions. When a confluency of $70-90 \%$ was reached the cells were transferred to the next bigger well until again a confluence of $70-90 \%$ was reached and so on (96-well $\rightarrow$ 24-well $\rightarrow 12$-well $\rightarrow 6$-well $\rightarrow$ T-25 flask), until finally the amount of cells was sufficient to fill a T-75 cell culture flask. Total protein was isolated from the cells and via western blot analysis the overexpression or downregulation of cyclin D2 within the transfected cells was tested. Cells transfected with pSingle-tTs-Ccnd2-shRNA had to be treated with doxycycline for $48-72$ hours prior to protein isolation in order to induce transcription of the shRNA. Cells that were tested positive for cyclin D2 overexpression or cyclin D2 downregulation were conserved by cryo-preservation and were subjected to proliferation, migration or soft agar assays. 


\subsubsection{Transfection of small interfering RNA (siRNA) into eukaryotic cells}

siRNAs were used for silencing of a specific gene. siRNAs bind to a specific mRNA, thereby marking it for degradation which results in a reduced gene expression. Three different mouse siRNAs against cyclin D2 (MSS236126, MSS236127, MSS236128, Stealth RNAi ${ }^{\text {TM }}$ siRNA, Life Technologies, Darmstadt) and three different human siRNAs against cyclin D2 were used (HSS101452, HSS101453, HSS101454, Stealth RNAi ${ }^{\text {TM }}$ siRNA, Life Technologies, Darmstadt). Human and mouse PCa cells were transfected with gene-specific siRNAs by using the siRNA transfection reagent Oligofectamine ${ }^{\mathrm{TM}}$ (Life Technologies, Darmstadt, Germany). Thereby complexes of lipids and siRNA oligonucleotides will be formed which facilitate the uptake of the siRNA molecule into mammalian cells. A sufficient amount of the cells were plated into a 6-well plate or T-25 culture flask so that the following day for transfection a confluence of $50 \%$ was reached. The transfection reaction was pipetted according to the manufacturer's instructions with a final concentration of $50 \mathrm{nM}$ siRNA- duplexes. Control PCa cells were transfected with siRNAduplexes against the luciferase gene Photinus pyralis. The transfection reaction was incubated for $30 \mathrm{~min}$ at RT in order to form the lipid-siRNA-complexes. In the meantime the cells were washed with DBPS (PAN, Aidenbach, Germany). OptiMEM I (Life Technologies, Darmstadt, Germany) was added to the cells as well the transfection reagent, which was applied dropwise. The cells were cultured overnight at normal culture conditions in the $37^{\circ} \mathrm{C}$ incubator. The following day the transfection reagent-containing medium was replaced by normal growth medium. Either protein or RNA was isolated from the transfected cells at different time points or the cells were further subjected to migration or soft agar assays.

\subsection{Functional analyses of eukaryotic cells}

\subsubsection{Migration assay}

48 hours prior to the in vitro cell migration assay NIH/3T3 cells were transfected with the cyclin D2-specific siRNAs. For the cell migration assay, 20000 cells suspended in $200 \mu$ medium were transferred to an insert of the assay (Millicell 8.0 $\mu \mathrm{m}$ hanging PET inserts, Millipore, Billerica, USA). The assay was incubated overnight at normal cell culture conditions. The following day, cells that had migrated through the membrane of the insert were stained using the Diff-Quick

Staining Set (Dade Behring $\mathrm{GmbH}$, Marburg, Germany) according to the manufacturer's instruction. The membranes were excised from the insert using a sterile scalpel and then fixed in oil on a slide. For evaluation, the number of cells that had migrated through the membrane 
overnight was counted per field of view of five pictures taken on the microscope (BX60, Olympus $\mathrm{GmbH}$, Hamburg, Germany) at a 40x magnification.

Cells transfected with pIRES2-EGFP-CCND2 or doxycycline-treated (48 hours) pEBTetDCCND2-transfected cells were plated right away into the inserts from Corning Transwell Permeable Supports (8.0 $\mu \mathrm{m}$ pore size and $6.5 \mathrm{~mm}$ Transwell insert diameter, Corning Inc., New York, USA). 20000 cells suspended in $100 \mu \mathrm{l}$ medium were transferred to the insert according to the manufacturer instruction. Cells were incubated overnight, stained, fixed and counted as described above.

\subsubsection{Proliferation assay}

The proliferation rate of PCa cells with cyclin D2 overexpression (transfected with pIRES2EGFP-CCND2 or pEBTetD-CCND2) or of NIH/3T3 cells with cyclin D2 downregulation (transfected with pSingle-tTs-Ccnd2-shRNA or siRNA) was studied. Therefore, the CellTiter 96® AQueous Non-Radioactive Cell Proliferation Assay (MTS, Promega, Mannheim, Germany) was used. This assay is based on the principle that faintly yellow stained tetrazoles (MTS) can be reduced to intensively purple stained formazan derivatives by living cells. MTS, in the presence of phenazine methosulfate (PMS), produces a formazan product that has an absorbance maximum at $490-500 \mathrm{~nm}$. The intensity of the staining is measured from which conclusions on the cell proliferation are drawn. This reaction requires active mitochondria which are found only in living cells. 3000 cells were plated into a well of a flat-bottomed 96-well cell culture plate. In case of siRNA-transfected 2E, PC-3, LNCaP and DU145 cells, which were also treated with VPA to study the functional consequence, the day following transfection 10000 cells suspended in VPA-containing medium were plated per well. After two hours, when the cells were adherent, the PMS/MTS solution was added according to the manufacturer's instruction. In case of cells transfected with the tetracycline inducible vectors pEBTetD and pSingle-tTs-shRNA doxycycline was simultaneously added. Cells were incubated in the PMS/MTS solution for 2 hours at $37^{\circ} \mathrm{C}$ and $5 \% \mathrm{CO}_{2}$ to let the color reaction develop. Then the intensity of the staining was measured using the SynergyMx plate reader spectrophotometer (BioTek, Friedrichshall, Germany). The generated data was transferred to MS Exel (Microsoft) for further calculation of the absolute and relative proliferation rate at a certain time point $(\mathrm{t})$ according to the following formulas:

Absolute Proliferation $(\mathrm{t})$ : $\quad$ mean $(\mathrm{t}) /$ mean $(\mathrm{t}=0)$

Relative Proliferation: absolute proliferation ( $\mathrm{t}$ )/absolute proliferation of the control (t) 


\subsubsection{Soft agar assay}

The soft agar assay is a detection method for the ability of cells to grow anchorage-independent in agarose in vitro. Transformed cells have the ability to grow independently of a solid surface, which is a hallmark of carcinogenesis (Borowicz et al. 2014). By contrast, mouse fibroblast cells $\mathrm{NIH/3T3}$ are not able to grow anchorage-independent. Therefore, these cells were tested after cyclin D2 siRNA-transfection or transfection with the pSingle-tTs-Ccnd2-shRNA plasmid for malignant transformation by using the soft agar assay.

A $5 \%$ agar solution was prepared with DPBS and sterilized by autoclaving. This was the basic solution for the different agar concentrations needed. The agar was liquefied in a microwave and kept for the duration of the experiment at a $45^{\circ} \mathrm{C}$ water bath. Through careful but rapid pipetting, the appropriate amount of $5 \%$ agar was mixed with pre-warmed cell culture medium to yield the agar concentration needed. The bottom of 6 -well plates was covered with $1 \mathrm{ml}$ of the so called bottom layer which consisted of $1 \%$ agar. The agar was applied carefully to avoid air bubbles. The plate was set aside for the bottom layer to solidify. Meanwhile, the top layer was prepared for which 5000 cells were added to $500 \mu$ l pre-warmed medium and then mixed with $500 \mu$ of $0.6 \%$ agar to yield a $1 \mathrm{ml}$ volume with $0.3 \%$ agar concentration for the top layer. It was applied carefully but rapidly to the bottom layer and then the plate was kept for 10 min at RT before it was transferred to the incubator with normal cell culture conditions. There it was kept for up to four weeks so that colonies could form from the single cells. For visualization of colonies the agar was stained overnight with $0.001 \%$ crystal violet.

The NIH/3T3 cells used for this experiment were transfected 48 hours prior to the soft agar with cyclin D2-specific siRNA. Cells from the pSingle-tTs-Ccnd2-shRNA transfected NIH/3T3 clone No. 11 were either treated with doxycycline 48 hours before the soft agar assay or they were transfected with pEGFP, pEGFP-KRAS or pEGFP-KRAS-G12V and treated the following day with doxycycline before they were subject to the soft agar assay.

\subsection{Immunohistochemistry}

\subsubsection{Immunofluorescent staining of eukaryotic cells}

For the immunofluorescent staining the cells were plated into 4-chamber slides at a medium cell density of 25000 cells per chamber. The cells were cultured overnight in normal cell culture medium at $37^{\circ} \mathrm{C}$ and $5 \% \mathrm{CO}_{2}$. The following day the cells were fixed with $3.7 \%$ formaldehyde in DPBS for $20 \mathrm{~min}$ at RT. Cells were washed briefly with DPBS and then incubated for $10 \mathrm{~min}$ 
with $0.1 \%$ Triton-X-100 in DPBS to make the membrane permeable. After the cells were washed again with DPBS they were blocked for 20 - 30 min with blocking buffer II to block unspecific binding sites. The cells were incubated at $4^{\circ} \mathrm{C}$ overnight with the primary antibody (anti-cyclin D2 (M-20), Santa Cruz, Heidelberg, Germany) which was diluted 1:200 with blocking buffer II. The next day the cells were washed twice for 10 min with DPBS. Afterwards the secondary Cy3-coupled antibody, which was diluted 1:200 with blocking buffer II, was applied to the cells and incubated for 2 hours. The cells were washed twice for $10 \mathrm{~min}$ with DPBS and once for $10 \mathrm{~min}$ with $\mathrm{H}_{2} \mathrm{O}$. Finally, the slides were covered with Vectashield/DAPI (VectorLab, Burlingame, USA) and the staining was documented with the fluorescence microscope BX60 (Olympus GmbH, Hamburg, Germany).

\subsubsection{Immunohistochemical staining of cryo-sections from CAM tumors}

The cryo-slides were removed from the $-20^{\circ} \mathrm{C}$ freezer and kept for 1 hour at RT to dry. Unspecific binding sides were blocked by incubation with goat blocking reagent for 1 hour at RT. The primary antibodies, Mep21 (chicken CD34 homolog; M. Williams, AbLab, University of British Columbia, Vancouver, B.C., Canada) and Prox1 (ReliaTech GmbH, Wolfenbüttel, Germany) were diluted 1:500 with antibody solution. The slides were incubated overnight at $4^{\circ} \mathrm{C}$ with the primary antibodies. The following day the slides were briefly washed twice with PBS before the secondary antibodies were added, which were diluted 1:200 with antibody solution. Secondary antibodies used were Alexa 594-conjugated goat-anti-rabbit IgG (Life Technologies, Darmstadt, Germany) and Alexa 488-conjugated goat-anti-mouse IgG1 (Life Technologies, Darmstadt, Germany). DAPI (kept in aliquots of 1:20000 dilution in PBS) was also added to the secondary antibody solution in a 1:100 dilution. The slides were incubated for $90 \mathrm{~min}$ in the dark at RT with the secondary antibody/DAPI. Afterwards they were washed twice with PBS. Finally, the slides were covered with Fluoromount-G (Sigma-Aldrich Chemie $\mathrm{GmbH}$, Taufkirchen, Germany) and the staining was documented with a microscope.

\subsection{In vivo studies}

The experiments were performed according to the guidelines of the European Parliament (2010/63/EU) and the council for the protection of animals in science ( $\$ 14$ TierSchVersV). 


\subsubsection{In vivo treatment of TRAMP mice with VPA}

TRAMP mice received VPA over the drinking water. A final concentration of $0.4 \% \mathrm{w} / \mathrm{v}$ VPA was applied to the mice. Since the final concentration was not tolerated instantly by the mice, VPA had to be crept in during the first week by increasing the concentrationd from $0.1 \% \mathrm{w} / \mathrm{V}$ VPA in $0.1 \%$ - steps every other day until the final concentration of $0.4 \% \mathrm{w} / \mathrm{v}$ was reached. To make the drinking water more tasty, common household sweetner tablets from the brand "Das gesunde PLUS" (dm-drogerie markt GmbH + Co. KG, Karlsruhe, Germany) were added to the drinking water (1 tablet/100 $\mathrm{ml}$ drinking water). Mice that received drinking water only with sweetner served as controls. This experiment consisted of 11 VPA-treated and 11 controltreated TRAMP mice. In Figure 2.1 the treatment scheme is depicted.

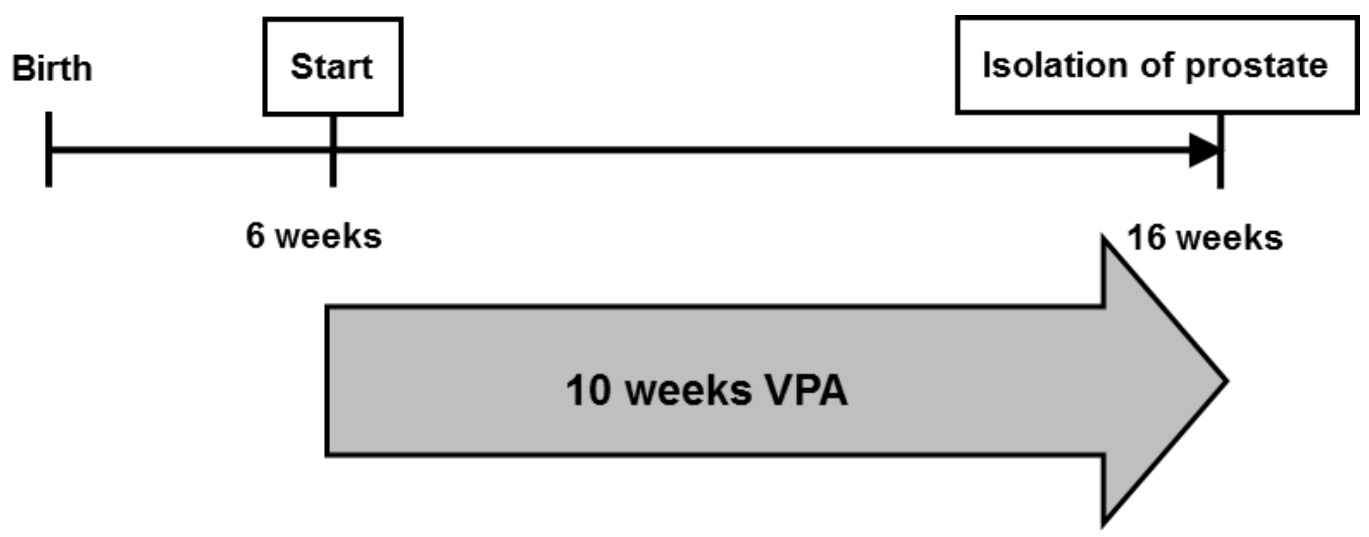

Figure 2.1: In vivo VPA treatment scheme. VPA treatment started when TRAMP mice were 6 weeks old. Both groups received their treatment for the duration of 10 weeks. At the age of 16 weeks the mice were sacrificed, their prostates were isolated and stored at $-80^{\circ} \mathrm{C}$ for molecular evaluation either by western blot analysis or quantitative real-time PCR.

\subsubsection{Chicken chorioallantoic membrane (CAM) assay}

The CAM assay was conducted in collaboration with Prof. Dr. Jörg Wilting in the Center of Anatomy, University Medical Center Göttingen. Specific pathogen-free fertilized White Leghorn chicken eggs were purchased from Valo BioMedia GmbH (Osterholz-Scharmbeck. Germany) and stored for up to 10 days in the refrigerator until the experiment was started. When beginning the experiment, the eggs were incubated for 72 hours at $>80 \%$ relative humidity and $37.8^{\circ} \mathrm{C}$. On developmental day 3 , a little window $(0.5 \mathrm{~cm} \times 0.5 \mathrm{~cm})$ was sawed into the egg shell and afterwards sealed again with cellotape. The eggs were thereafter incubated again for seven days. On developmental day 10, the window was re-opened and $50 \mu \mathrm{l}$ of PCa cells-matrigel mixture were applied to the CAM. For the murine PCa $2 \mathrm{E}$ cells, $2 \times 10^{6}$ cells were applied per 
egg and for the human PCa PC-3 cells $5 \times 10^{6}$ cells were applied per egg. The cells were resuspended in $50 \%$ normal growth medium and $50 \%$ Matrigel (Corning $®$ Matrigel $\circledast$ Basement Membrane Matrix, LDEV-Free, Product \#354234, Corning Inc., New York, USA). Before application to the CAM, the cells were pre-treated with either $1 \mathrm{mM}$ or $3 \mathrm{mM}$ VPA for 24 hours or seven days. On the day of application to the CAM the same concentration of VPA was added to the medium-Matrigel mixture. Control cells were not treated with VPA at all. After adding the cells to the CAM the window was sealed again with cellotape and the eggs were further incubated for seven days. Cells that were treated with VPA were re-treated after three days by applying $10 \mu \mathrm{l}$ of cell culture medium with the appropriate amount of VPA onto the already developed tumor within the egg. On developmental day 17 the window was re-opened and enlarged so that the tumors that had grown from the applied cells could be excised. Tumors that were subjected to embedding and immunohistochemical analysis were first fixed with $4 \%$ paraformaldehyde within the egg for $20 \mathrm{~min}$. Tumors that were subjected to gene expression analyses by quantitative real-time PCR were excised right away without fixation, transferred to liquid nitrogen and then stored at $-80^{\circ} \mathrm{C}$ until continuation with total RNA isolation. The fixed tumors were excised from the CAM, washed with PBS and then pictures were taken with the Leica MZ16FA microscope. Afterwards, the tumors were placed into little tubes filled with $10 \%$ sucrose (in PBS). When the tumors were soaked with the sucrose they sank to the bottom. Only then the tumors were transferred for overnight incubation at $4^{\circ} \mathrm{C}$ to a tube filled with $30 \%$ sucrose (in PBS). The following day tumors were embedded in clear PolyFreeze Tissue Freezing Medium (Polysciences Europe $\mathrm{GmbH}$, Eppelheim, Germany) and stored for one night at $-80^{\circ} \mathrm{C}$ before they were kept for long-term storage at $-20^{\circ} \mathrm{C}$. The embedded tissue was cut with a cryotome (Leica CM3050 S, Wetzlar, Germany) into $16 \mu \mathrm{m}$ thick sections. Three to four sections were transferred to one slide. Until the slides were used for immunofluorescent staining they were stored at $-20^{\circ} \mathrm{C}$.

\subsection{Statistics}

To analyze the statistical significance of the experimental results the unpaired $t$ test was used.

The results were depicted as follows:

$$
\begin{aligned}
& { }^{*} 0.01<p \leq 0.05, \text { "significant" } \\
& { }^{* *} 0.001 \leq p<0.01, \text { "very significant" } \\
& { }^{* * *} p \leq 0.001, \text { "extremely significant" }
\end{aligned}
$$




\section{Results}

\subsection{Molecular effects of VPA treatment in PCa therapy}

Witt et al. could show that VPA treatment of the murine 2E PCa cells reduced the migration, invasion and proliferation rate, all in a concentration-dependent manner (Witt et al., 2013). In vivo studies showed that TRAMP mice that received VPA with the drinking water had a reduced number and onset of tumors as well as an increased age of survival compared to control-treated mice (Witt, 2012). Microarray analysis of 2E cells treated for 24 hours with VPA identified several candidate genes that could be responsible for the observed effects of VPA treatment in vivo and in vitro (Witt, 2009). Eight candidate genes were chosen for further investigation because of their known expression in the prostate and their known connection to cancer. They included the downregulated genes chemokine (C-X-C motif) ligand 15 ( $C x \mathrm{x} / 15)$, which codes for a cytokine of the CXC chemokine family, RCC1 and BTB domain-containing protein 2 (Rcbtb2) coding a member of the RCC1 related GEF family, both transcript variants of ceruloplasmin $(C p)$, which is the main copper transporting protein of blood, and leukemia inhibitory factor (Lif), which codes for a class 6 interleukin. Candidate genes that were upregulated after VPA treatment included ubiquitin carboxy-terminal hydrolase L1 (Uch/1), which codes for a de-ubiquitinising enzyme, tyrosine-protein phosphatase-like N (Ptprn), which codes for a member of the protein tyrosine phosphatase family and cyclin $D 2$ ( $C c n d 2)$, which codes for a member of the strongly conserved D-type cyclin family. The up- and downregulation of the candidate genes was verified by quantitative real-time PCR on cDNA derived from VPA-treated 2E cells (Witt et al., 2013). In the present project TRAMP mouse tumors which were derived and isolated from previous in vivo VPA experiments (Witt, 2012) were now further evaluated in terms of deregulated candidate gene expression.

\subsubsection{Verification of deregulated candidate gene expression in tumors of VPA-treated TRAMP mice}

The expression of the candidate genes was investigated both on RNA and protein level in prostate tumors derived of VPA- and control-treated TRAMP mice.

This first in vivo VPA treatment experiment was conducted by Witt during her dissertation (2012). Witt used three different study groups which were treated with a final concentration of $0.4 \% \mathrm{w} / \mathrm{v}$ VPA and sweetener over the drinking water. 
Control-treated mice received drinking water with sweetener only. Two of the study groups were preventive study groups which received VPA treatment at the age of 6 weeks when the prostate shows no malignant changes yet. One of the groups was treated until the age of 30 weeks (group 1), when tumor progression should still be ongoing, and then sacrificed. The second group was treated until the age of 40 weeks (group 2), when TRAMP mice should have developed a PCa, and then sacrificed. The third group received VPA as a curative treatment starting at the age of 16 weeks, when tumor development should have initiated already in TRAMP mice, and at the age of 40 weeks they were sacrificed (group 3, Mentor-Marcel et al., 2001). If mice showed a weight loss of more than $20 \%$ within 48 hours, exhibited behavioral problems (including apathy, increased aggressiveness, paralysis) or had a clearly palpable PCa, the mice were sacrificed immediately. Of all the sacrificed TRAMP mice the prostate tumors were isolated (Witt, 2012).

\subsubsection{Verification of deregulated candidate gene expression on RNA and protein level}

The expression of the candidate genes Cp1, Cp2, Ccnd2, Ptprn, Uch/1, Lif and Rcbtb2 was analyzed by quantitative real-time PCR on RNA derived from isolated prostate tumors of VPAand control-treated TRAMP mice. In total, 12 prostate tumors were available for molecular evaluation. Six of the tumors were derived from VPA-treated TRAMP mice (group 1: 1 tumor, group 2: 3 tumors, group 3: 2 tumors) and the other six tumors were received from control mice (group 1: 1 tumor, group 2: 4 tumors, group 3: 1 tumor).

In vivo VPA treatment led to a significant reduction of the Cp2 and Lif expression in prostate tumor tissue (Fig.3.1). Cp2 and Lif were also downregulated in the previous microarray experiment of VPA-treated 2E cells (Witt, 2009). The Ccnd2 expression after VPA treatment was not quite significantly decreased in the tumor tissue $(p=0.0511)$ which is contrary to the array result. The expression of Uchl1 and Ptprn was downregulated after VPA treatment, which is also contrary to the microarray results. Cp1 expression was downregulated after VPA treatment in the prostate tumors of TRAMP mice, though not statistically significant, which is in accordance to the microarray data. Rcbtb2 showed no expression differences in tumors of VPA and controltreated TRAMP mice (Fig 3.1).

By quantitative real-time PCR analysis on prostate tumor tissue the downregulation of three candidate genes could be confirmed ( $C p 1, C p 2$ and Lif) whereas three genes showed a contrary expression pattern (Ccnd2, Uchl1 and Ptprn) and Rcbtb2 expression was not deregulated. 

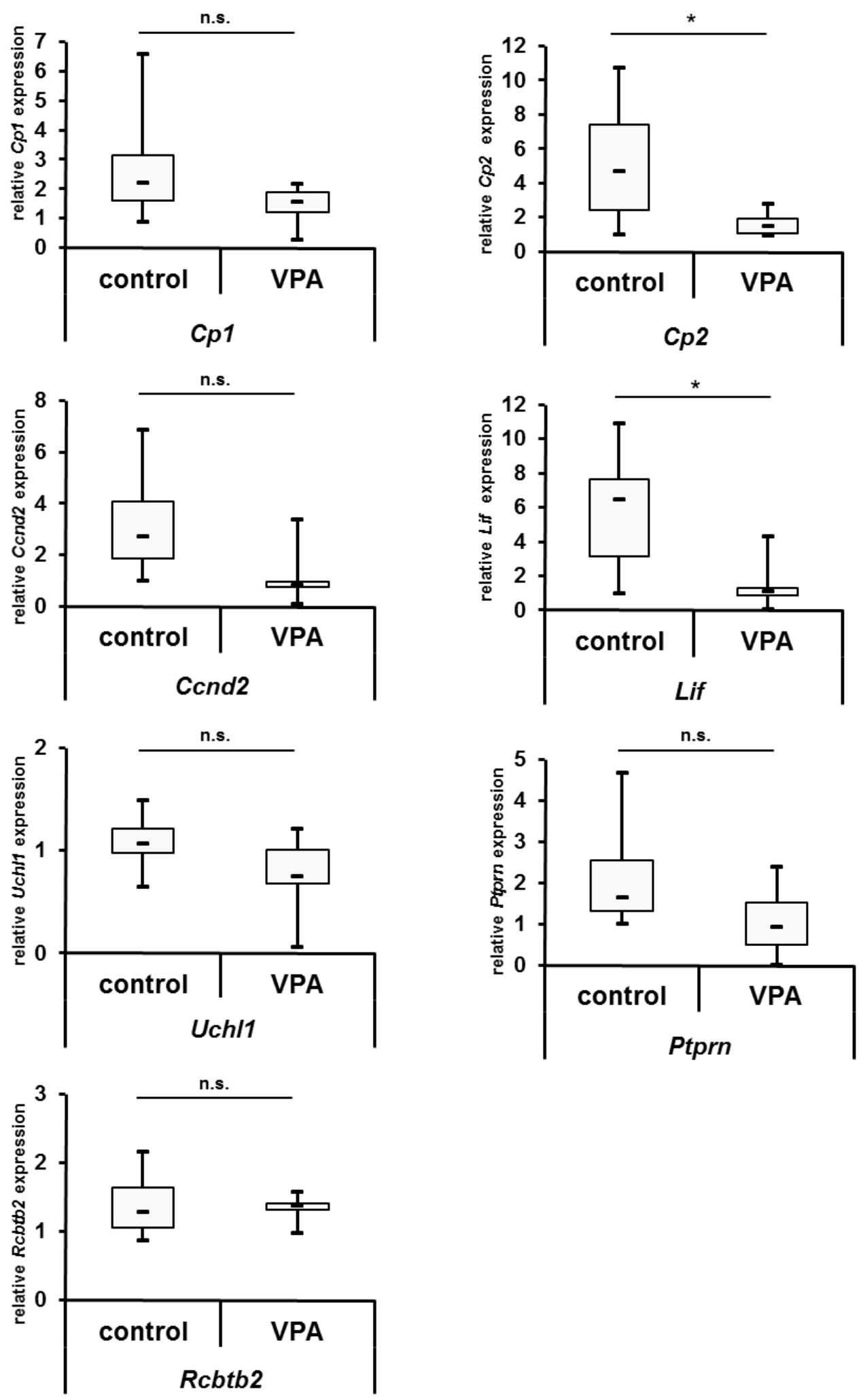

Fig. 3.1: Candidate gene expression analysis in tumor tissue of VPA-treated TRAMP mice. RNA was isolated from prostate tumors of in vivo VPA-treated TRAMP mice (6 tumors) and from the prostate tumors of control-treated TRAMP mice (6 tumors). RNA was reverse transcribed into cDNA and quantitative real-time PCR analysis was conducted. VPA treatment led to a significant reduction of $C p 2$ expression $(p=0.0349)$ and Lif expression $(p=0.0176)$ 
in prostate tumor tissue compared to tumor tissue of control-treated mice. The expression of Ccnd2, Cp1, Uch/1, Rcbtb2 and Ptprn was not statistically significant deregulated in prostate tumor tissue of VPA-treated and control TRAMP mice. Data are presented as mean +/- standard deviation of three quantitative real-time PCR analyses performed in triplicate. ${ }^{*} 0.01<p \leq 0.05,{ }^{* *} 0.001 \leq p<0.01,{ }^{* * *} p \leq 0.001$, n.s.: not significant

On the protein level the expression of cyclin D2 was tested by western blot analysis (Fig.3.2A). Densitometrical evaluation of a single western blot showed that there is a statistically significant increase in the cyclin D2 expression in the prostate tumors of VPA-treated TRAMP mice as compared to control-treated mice (Fig. 3.2B).
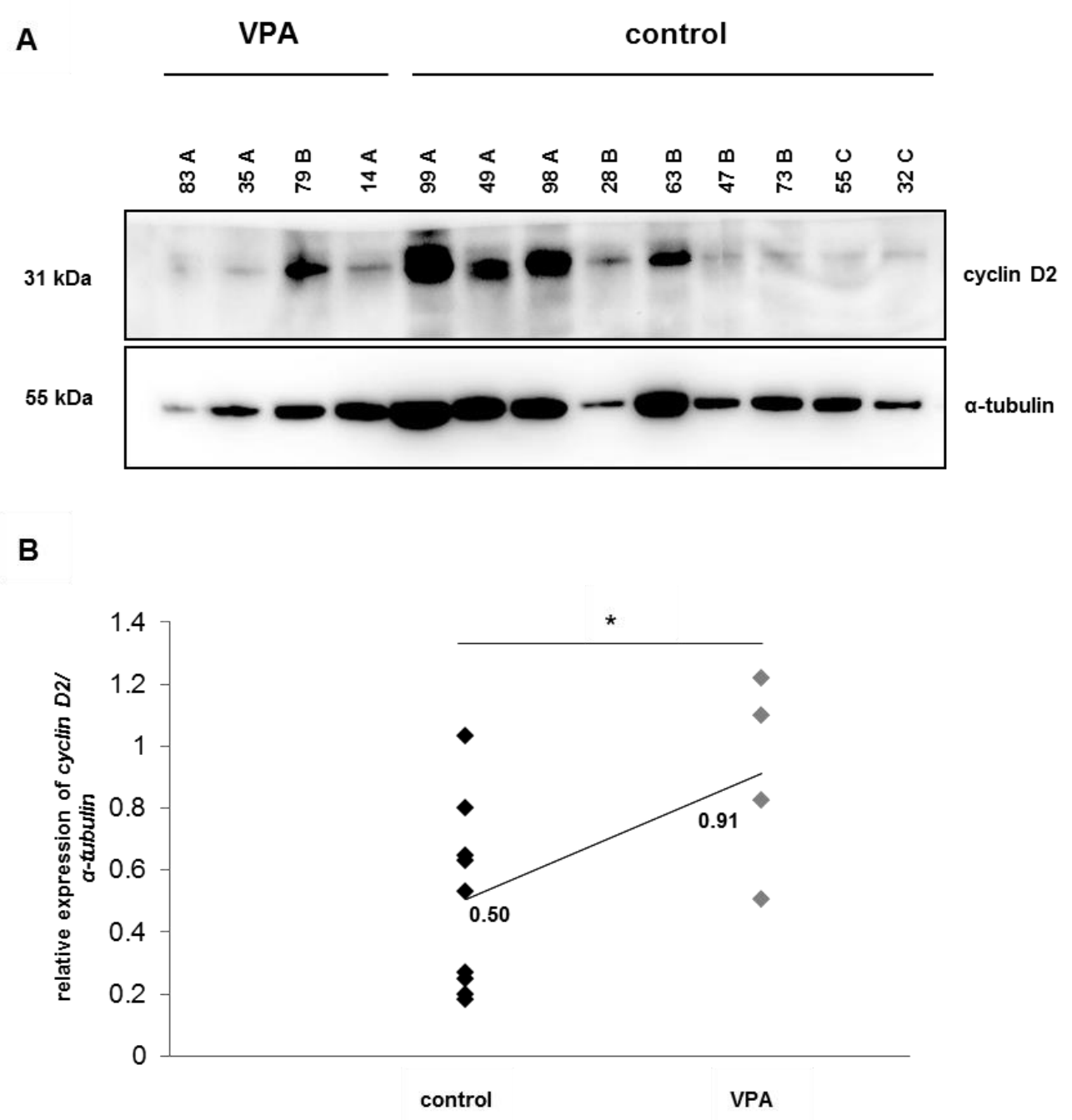

Fig. 3.2: Cyclin D2 expression in tumors of VPA and control-treated TRAMP mice. A) Whole protein lysate of prostate tumors derived from VPA-treated TRAMP mice was immunoblotted using a cyclin D2-specific antibody. Protein lysate of tumors derived from mice not treated with VPA served as controls. Immunoblotting of $\alpha$-tubulin ensured equal protein loading. B) Densitometrical analysis revealed a significant increase in the cyclin D2 expression in prostate tumors of VPA-treated mice as compared to prostate tumors derived from control mice $(p=0.0480)$. ${ }^{*} 0.01$ $<\mathrm{p} \leq 0.05,{ }^{* *} 0.001 \leq \mathrm{p}<0.01,{ }^{* * *} \mathrm{p} \leq 0.001$ 


\subsubsection{In vivo VPA treatment of TRAMP mice}

A new in vivo VPA treatment experiment of TRAMP mice was conducted due to the fact that in the previous in vivo experiment too many points were not consistent: tumors were isolated at different time points depending on either which of the three study groups the mice belonged to (two preventive groups sacrificed at weeks 30 or 40 and one curative group sacrificed also at week 40 but treatment started at week 16 and not at week 6 like for the other two groups), the size of the tumor or behavioral problems of the mice. The difference in the mice age when their prostate tumor was isolated could also lead to differential expression of the candidate genes. In addition, the few isolated tumors may have risen due to either failure of therapy or resistance to VPA treatment. To avoid the differences between mice mentioned above a new in vivo VPA treatment experiment of TRAMP mice was conducted. This time there was only one study group consisting of 11 VPA-treated and 11 control-treated mice. The treatment for the mice started at the age of 6 weeks. All mice were sacrificed at the age of 16 weeks, resulting in a 10 week treatment period (Fig. 2.1). The mice received 0.4\% w/v VPA with the drinking water which was enriched with sweetener. Control-treated mice received water with sweetener only. When the mice were sacrificed their prostates were isolated for molecular evaluation. None of the TRAMP mice had developed a visible prostate tumor yet.

The expression of the eight candidate genes from the microarray was analyzed on RNA derived from prostates of VPA- and control-treated TRAMP mice by quantitative real-time PCR. This analysis revealed a significant downregulation of $C p 2$ and a significant upregulation of Ptprn and Uchl1 in the prostate tissue of VPA-treated mice compared to prostate tissue of control-treated mice (Fig. 3.3). These results are in accordance to the microarray data (Witt, 2009). The other candidate genes showed no statistically significant expression differences in the prostates of VPA- and control-treated TRAMP mice although the expression of Rcbtb2 was not quite significantly upregulated after VPA treatment ( $p=0.0523$, Fig. 3.3). 

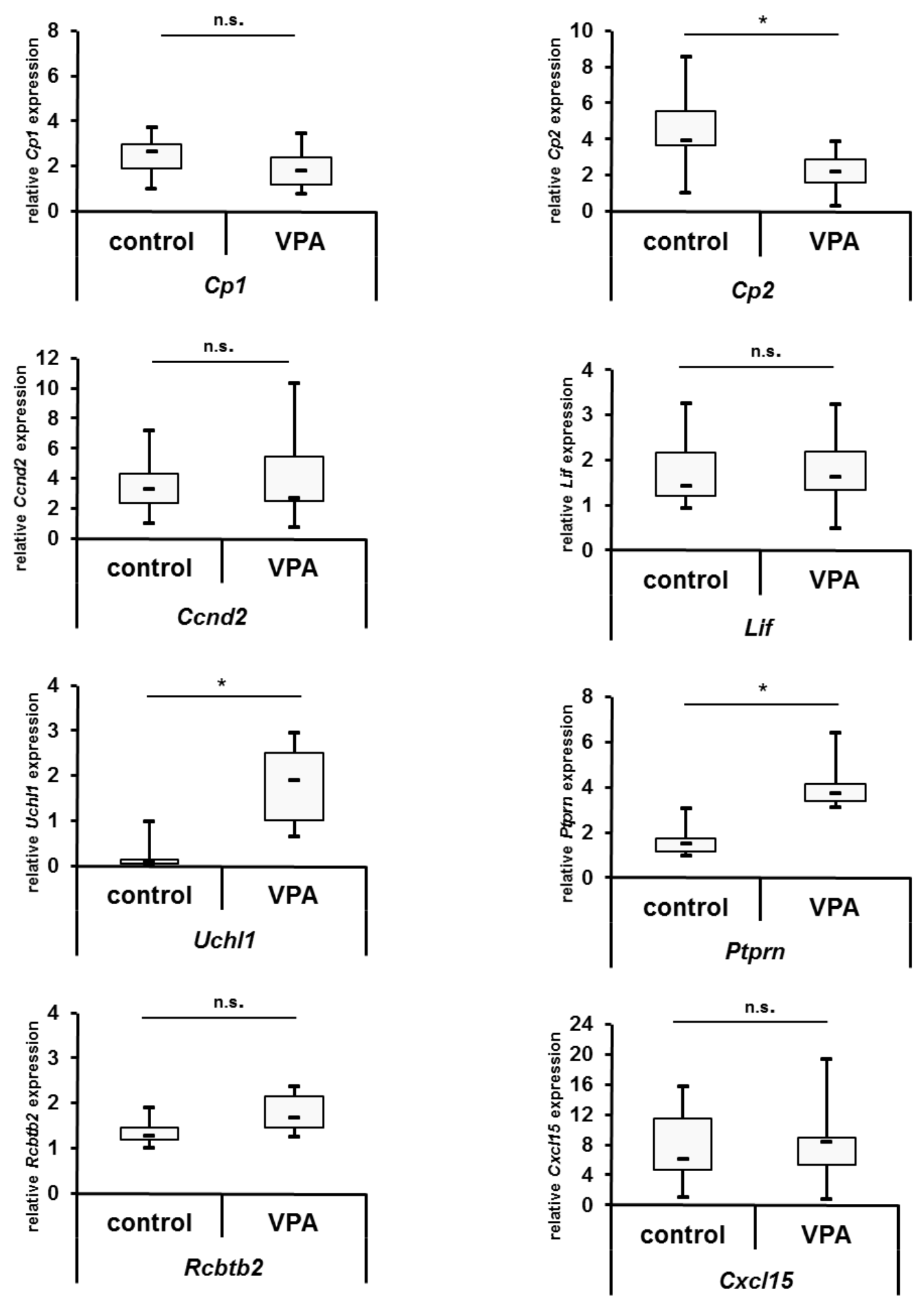

Fig. 3.3: Candidate gene expression analysis in prostate tissue of VPA-treated TRAMP mice. RNA was isolated from prostate tissue of 11 TRAMP mice that were treated for 10 weeks with VPA over the drinking water and from 11 control-treated TRAMP mice. RNA was reverse transcribed into CDNA and quantitative real-time PCR analysis was conducted. VPA treatment led to a significant reduction of $C p 2$ expression $(p=0.0451)$ and a significant increase in Ptprn and Uchl1 expression ( $\mathrm{p}=0.0011 ; \mathrm{p}=0.0010)$ in the prostate tissue of TRAMP mice as compared to prostate 
tissue of control-treated TRAMP mice. The expression of Ccnd2, Cp1, Rcbtb2, Lif and Cxcl15 was not statistically significant deregulated in prostate tissue of VPA-treated TRAMP mice compared to prostate tissue of control-treated TRAMP mice. Data are presented as mean +/- standard deviation of three quantitative real-time PCR analyses performed in triplicate. ${ }^{*} 0.01<p \leq 0.05,{ }^{* *} 0.001 \leq p<0.01,{ }^{* * *} p \leq 0.001$, n.s.: not significant

\subsubsection{Ceruloplasmin expression in human PCa cells}

The expression of the ceruloplasmin $(C P)$ gene was investigated in human PCa cells $\mathrm{LNCaP}$, DU145 and PC-3 because to date CP expression was only studied in mouse PCa 2E cells (Witt, 2009). The PCa cells were treated in vitro for 72 and 144 hours with VPA, respectively, before RNA was isolated. Untreated PCa cells served as controls. The RNA was reversed transcribed into CDNA and the expression of $C P$ was analyzed by quantitative real-time PCR. In the human genome only one isoform of $C P$ exists whereas in mouse two transcript variants are known. For all three human PCa cell lines a reduced $C P$ expression was observed after VPA treatment, mostly in a time- and concentration-dependent manner (Fig.3.4A). To confirm the quantitative real-time PCR result also at protein level western blot analyses for $\mathrm{CP}$ expression in murine and human PCa cells was performed. In 2E cells the protein level of CP did not change upon VPA treatment and in human PCa cells DU145, LNCaP and PC-3 the CP level was rather increased after VPA treatment (Fig.3.4B, depicted only for 2E and DU145 cells). Because the western blot analyses could not confirm the quantitative real-time PCR results, ceruloplasmin expression studies in PCa cells were discontinued. 

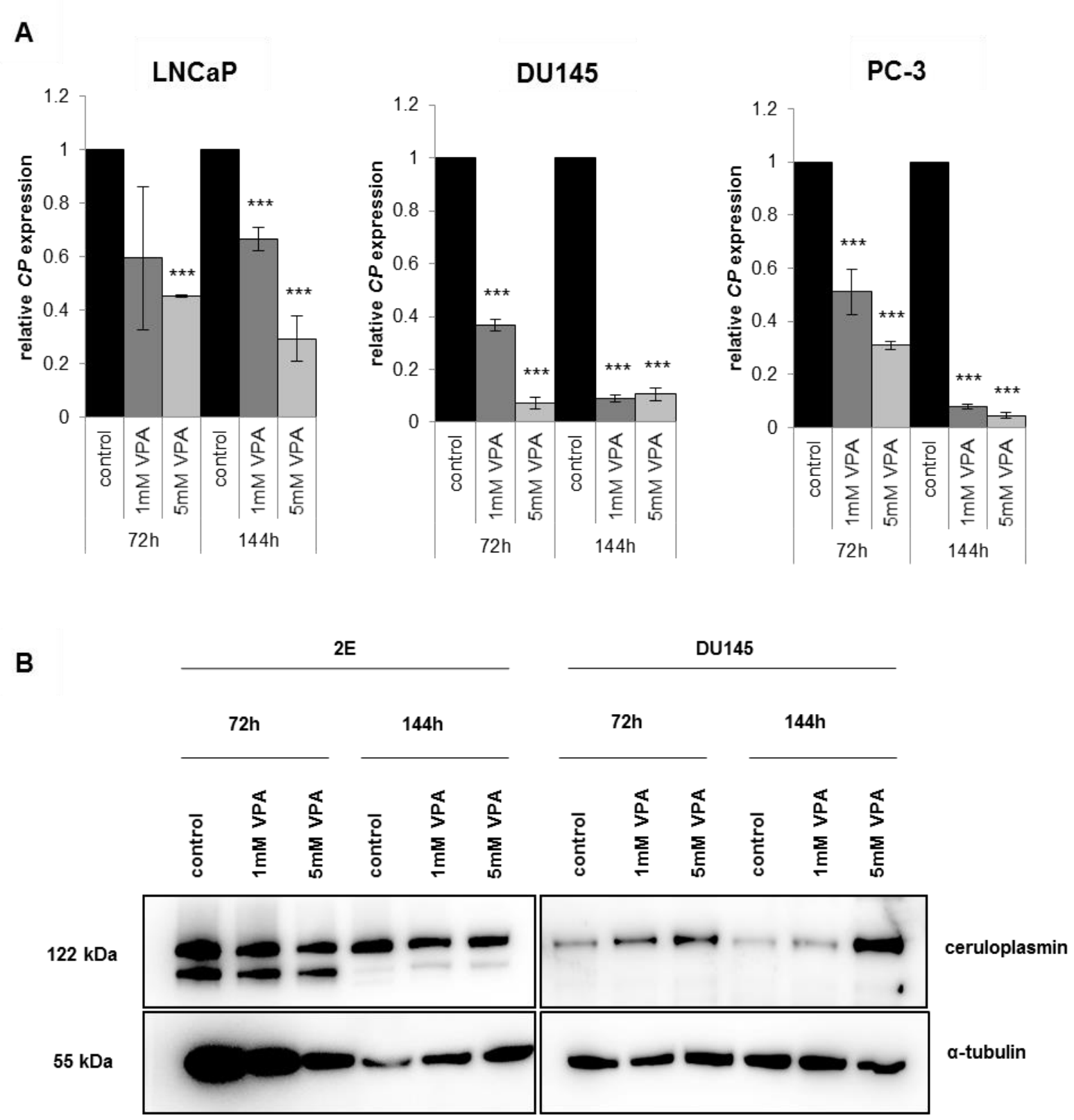

Fig. 3.4: Ceruloplasmin expression in human PCa cells. The human PCa cells LNCaP, DU145 and PC-3 were treated for 72 and 144 hours with two concentrations of VPA $(1 \mathrm{mM}$ and $5 \mathrm{mM})$ in vitro. Protein lysate and total RNA was isolated and the RNA was reverse transcribed into cDNA. The generated CDNA was subjected to subsequent quantitative real-time PCR analyses. A) For all three human PCa cell lines a reduced $C P$ expression was observed after VPA treatment, mostly in a time- and concentration-dependent manner as compared to untreated cells. B) Protein lysate was subjected to western blot analysis using a ceruloplasmin-specific antibody. Immunoblotting of $\alpha$ tubulin ensured equal protein loading. In 2E cells the ceruloplasmin protein level of VPA-treated cells was comparable to controls whereas in human PCa cells DU145 the ceruloplasmin level increased after VPA treatment. This was also observed for the human PCa cells LNCaP and PC-3 (data not shown). Data are presented as mean +/- standard deviation of three quantitative real-time PCR analyses performed in triplicate. ${ }^{*} 0.01<p \leq 0.05,{ }^{* *} 0.001 \leq p<0.01$,

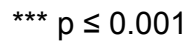




\subsubsection{Effects of VPA treatment on the expression of angiogenesis markers}

Since three of the eight candidate genes from the previous microarray experiment (Witt, 2009), namely $C p 1, C p 2$ and $C x c / 15$, are associated with angiogenesis the effects of VPA treatment on angiogenesis were analyzed in the present study in more detail. Therefore, the expression of several so called angiogenesis markers was investigated by quantitative real-time PCR first in in vitro VPA-treated 2E PCa cells and then in prostate tumors and prostate tissue of in vivo VPAtreated TRAMP mice. The angiogenesis markers were chosen from a publication by Shih et al. and included two angiogenic cytokines (vascular endothelial growth factor $A$ (Vegfa) and angiopoietin 1 (Ang1), three endothelial cell receptor tyrosine kinases (vascular endothelial

growth factor receptor 1 (Flt-1/Vegfr1), vascular endothelial growth factor receptor 2 (Kdr/Vegfr2), tyrosine kinase with immunoglobulin-like and EGF-like domains 1 (Tie-1)), and an endothelial cell adhesion molecule, platelet endothelial cell adhesion molecule (Pecam-1, Shih et al., 2002).

\subsubsection{Effect of in vitro VPA treatment on the expression of blood- and lymph- angiogenesis markers}

The mouse PCa cells 2E were treated for 72 and 144 hours with two different concentrations of VPA (1mM and 5mM). RNA was isolated and reversed transcribed into cDNA. The cDNA was used for the subsequent quantitative real-time PCR analyses to test the expression of the angiogenesis markers Vegfa, Ang1, Flt-1/Vegfr1, Kdr/Vegfr2, Tie-1 and Pecam-1. Cells that were not treated with VPA served as controls.

The angiogenic cytokines Vegfa and Ang1 as well as the receptor tyrosine kinase Tie-1 and the endothelial cell adhesion molecule Pecam-1 were statistically significant downregulated in $2 \mathrm{E}$ cells that were treated with VPA as compared to control treated cells (Fig. 3.5). The decrease in the expression was concentration-dependent and partially also time-dependent (Fig. 3.5). However, the two receptor tyrosine kinases Flt-1/Vegfr1 and Kdr/Vegfr2 were statistically significant upregulated after VPA treatment as compared to untreated control cells. 

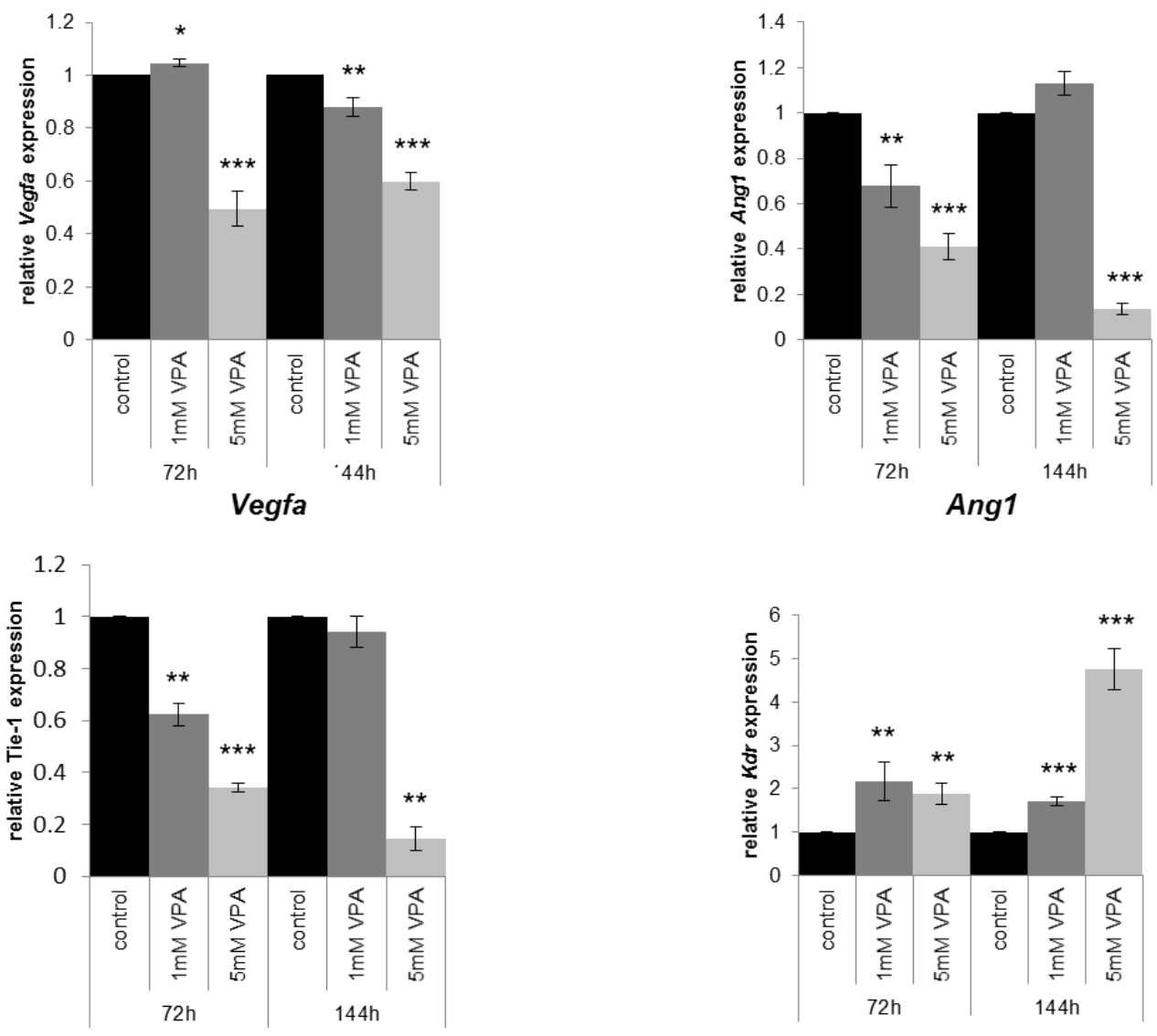

Tie-1

KdrlVegfr2

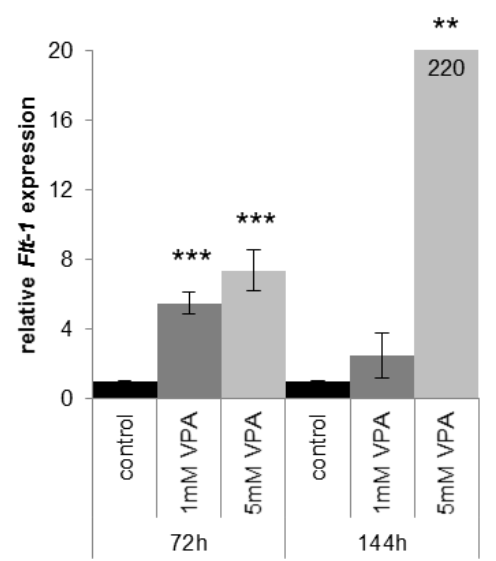

Flt-1/Vegfr1

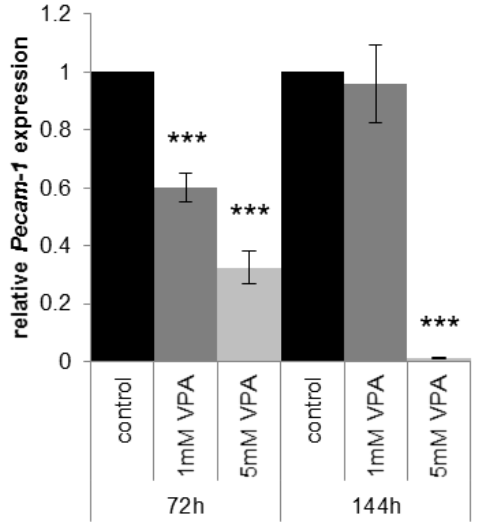

Pecam-1

Fig. 3.5: Expression analyses of angiogenesis markers in PCa cells after VPA treatment. The mouse PCa 2E cells were treated for 72 and 144 hours with either $1 \mathrm{mM}$ or $5 \mathrm{mM}$ VPA in vitro. Cells that were not treated with VPA served as control. RNA was isolated from which cDNA was generated by reverse transcription. Using this cDNA the expression of the following six angiogenesis markers was analyzed by quantitative real-time PCR analysis: two angiogenic cytokines (Vegfa and Ang1), three endothelial cell receptor tyrosine kinases (Flt-1/Vegfr1, Kdr/ Vegfr2, Tie1), and one endothelial cell adhesion molecule (Pecam-1). In vitro VPA treatment resulted in a decreased expression of Vegfa, Ang1, Tie-1 and Pecam-1. The downregulation of these genes after VPA treatment was concentration- and also mostly time-dependent. The two receptor tyrosine kinases Flt-1/Vegfr1 and Kdr/Vegfr2 had an increased 
expression after VPA treatment as compared to control cells. This effect was also concentration-dependent. Data are presented as mean $+/$ - standard deviation of three quantitative real-time PCR analyses performed in triplicate. ${ }^{*} 0.01$ $<p \leq 0.05,{ }^{* *} 0.001 \leq p<0.01,{ }^{* * *} p \leq 0.001$

Tumors grow and spread not only by blood angiogenesis but also by lymphangiogenesis. To differentiate between these two angiogenesis options the components of the VEGF-system are helpful because some components induce mainly blood angiogenesis, i.e. VEGFA when binding to the VEGFR2 receptor (Cursiefen et al., 2004). Other components induce mainly lymphangiogenesis, i.e. VEGFC when binding to VEGFR2 or VEGFR3 (Skobe et al., 2001) or inhibit lymphangiogenesis, like the soluble form of VEGFR2 (sVEGFR2, Albuquerque et al., 2009).

To study the effects of VPA treatment on blood- and lymphangiogenesis in vitro the expression of the different VEGF-system components was analyzed by quantitative real-time PCR. Therefore, the mouse PCa cells 2E and the human PCa cells PC-3 were treated for 72 and 144 hours with different VPA concentrations ( $1 \mathrm{mM}$ and $5 \mathrm{mM})$. RNA was isolated from the PCa cells and reverse transcribed into CDNA which was then used for quantitative real-time PCR analyses. The expression of the ligands Vegfa and Vegfc was analyzed as well as the expression of the receptors Flt-1/Vegfr1 and Kdr/Vegfr2 and of sVegfr2. Untreated PCa cells served as control. Some components of the VEGF-system were already analyzed for the murine 2E PCa cells within the set of angiogenesis markers (see Fig. 3.5) and are presented here again to visualize the relation of the different components of the VEGF-system.

In 2E PCa cells the expression of the ligands Vegfa and Vegfc was decreased, whereas the expression of the receptors Flt-1/Vegfr1 and Kdrl Vegfr2 increased in a concentration-dependent manner after VPA treatment as compared to untreated control cells (Fig. 3.6A). In human PC-3 PCa cells the expression of VEGFA decreased when cells were treated with $1 \mathrm{mM}$ VPA but increased after treatment with a $5 \mathrm{mM}$ concentration for 72 hours. After 144 hours of VPA treatment VEGFA expression was decreased compared to untreated PC-3 cells. The FLT1/VEGFR1 expression was decreased in PC-3 cells after VPA treatment for both time points. The VEGFC, KDR/VEGFR2 and sVEGFR2 expression was increased in PC-3 cells after VPA treatment for 72 hours as compared to untreated control cells. This effect was abolished after 144 hours of VPA treatment (Fig. 3.6B).

The differential expression of the VEGF-system components in PCa cells after VPA treatment compared to untreated cells highlights the beneficial effects of VPA treatment in PCa cells. Downregulation of the ligands and receptors of the VEGF-system after VPA treatment indicate that there is decreased expression of factors which play a role in blood or lymphangiogenesis. 
Also by upregulation of $S$ VEGFR2 there is a competition of VEGFC binding to either $K D R / V E G F R 2$ or sVEGFR2 which would also result in reduced lymphangiogenesis.

A
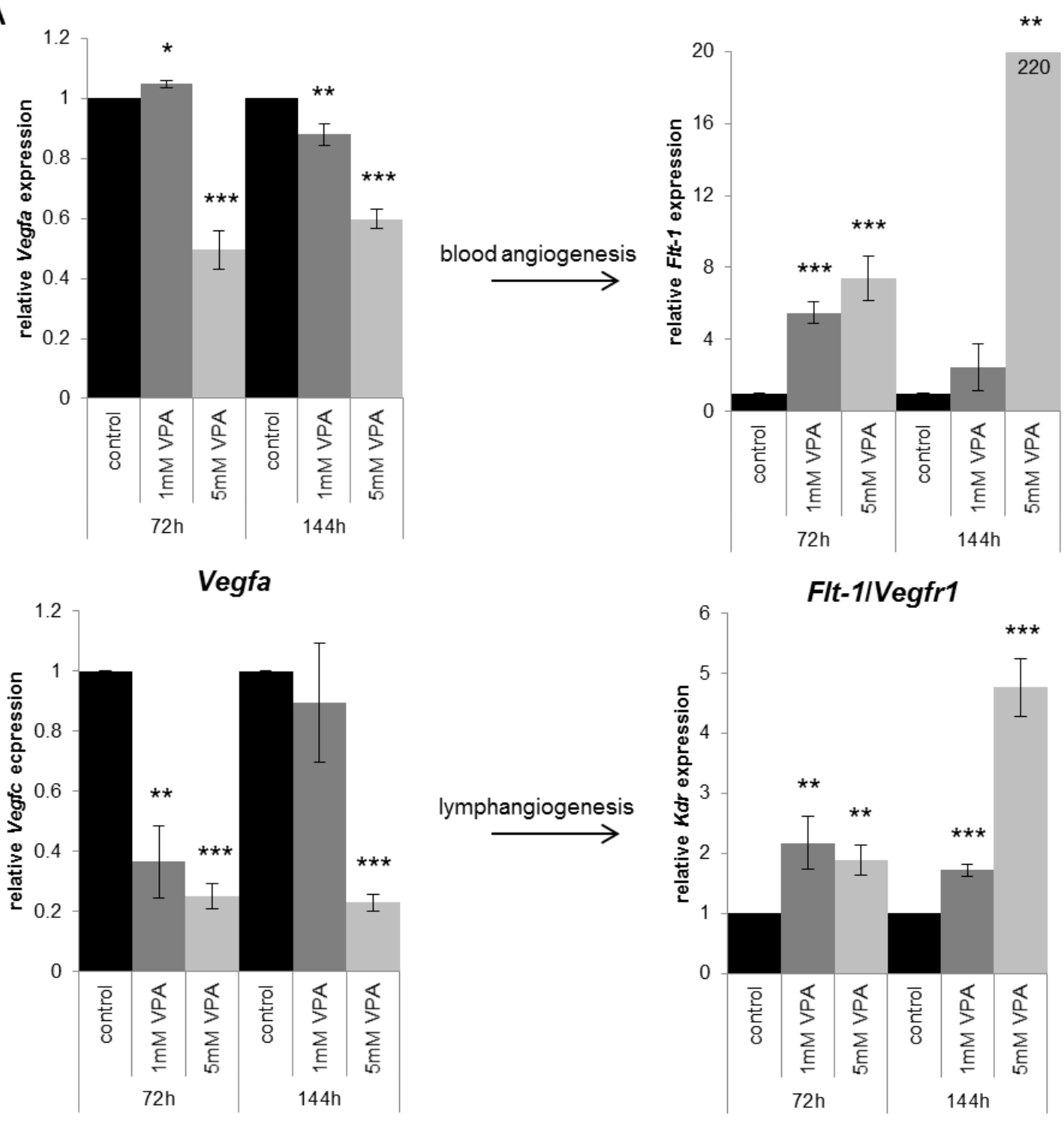

Vegfc

KdrlVegfr2 
B

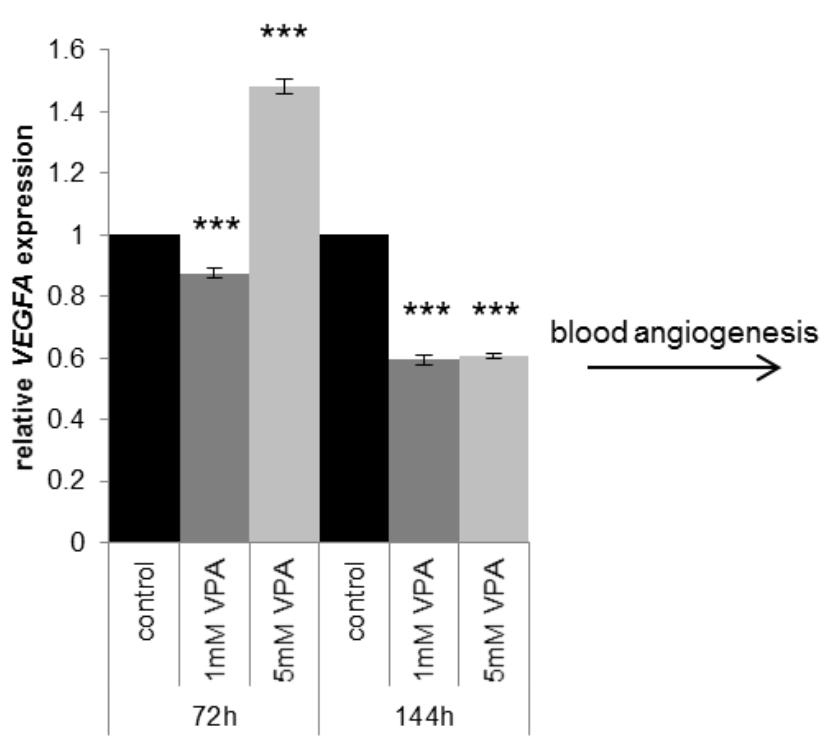

VEGFA

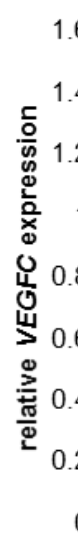

0

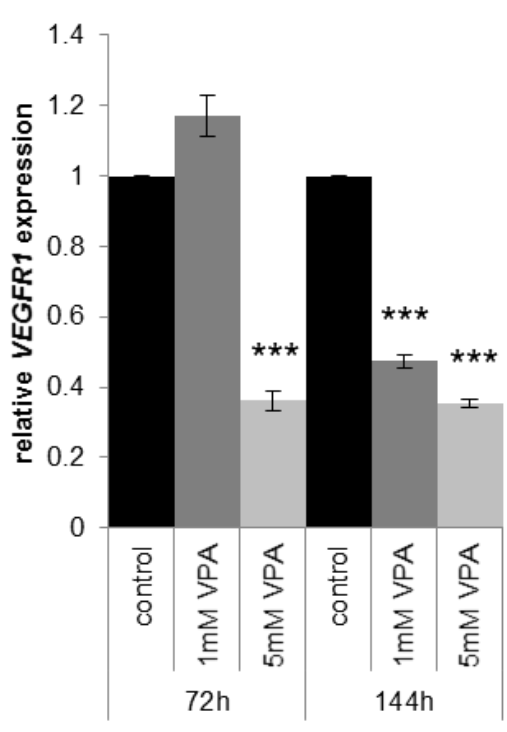

FLT-1IVEGFR1

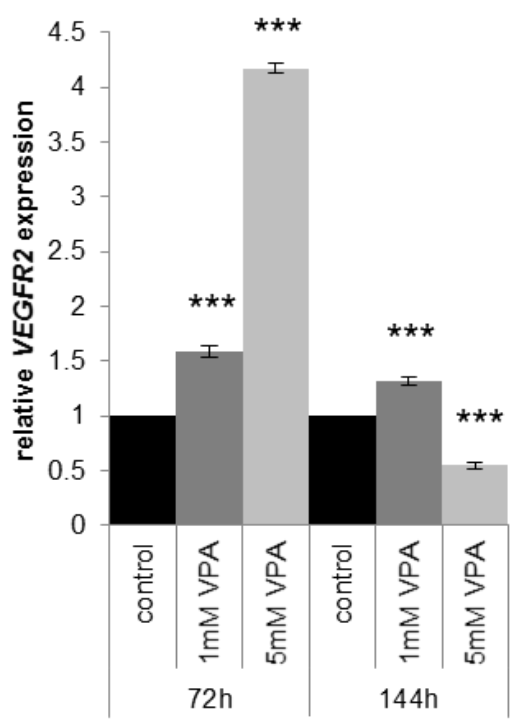

KDRIVEGFR2

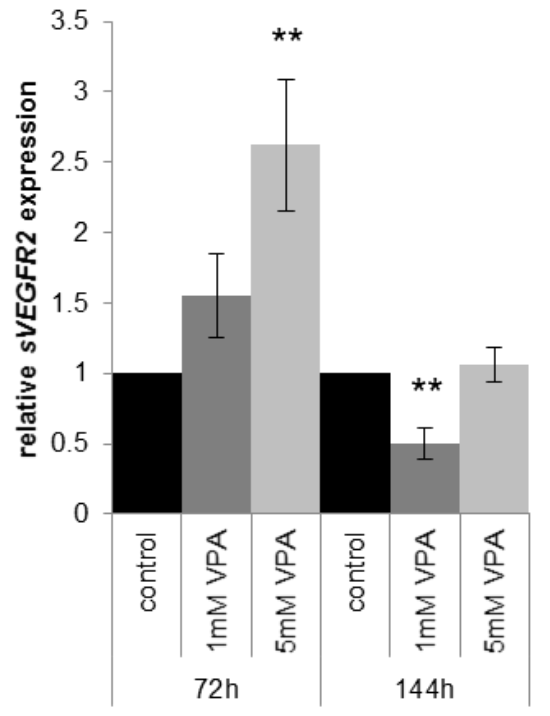

sVEGFR2 
Fig. 3.6: Expression analyses of VEGF-system components in PCa cells. The mouse $2 E P C a$ cells $(A)$ and the human PC-3 PCa cells (B) were treated for 72 hours and 144 hours with $1 \mathrm{mM}$ VPA and 5mM VPA, respectively. RNA was isolated and transcribed into CDNA on which the expression of some components of the VEGF-system was analyzed by quantitative real-time PCR. Cells without VPA treatment served as controls. A) In 2E cells the expression of Vegfa and Vegfc decreased in a concentration-dependent manner after VPA treatment as compared to untreated cells, whereas the expression of the receptors Flt-1/Vegfr1 and Kdr/Vegfr2 increased in a concentration-dependent manner after VPA treatment. B) In PC-3 cells the expression of VEGFA decreased when the cells were treated with $1 \mathrm{mM}$ VPA but increased after treatment with a $5 \mathrm{mM}$ concentration of VPA for 72 hours. After 144 hours of VPA treatment the VEGFA expression was decreased compared to untreated cells. The FLT-1/VEGFR1 expression was decreased after VPA treatment for both time points. The VEGFC, KDR/VEGFR2 and SVEGFR2 expression was increased after VPA treatment for 72 hours as compared to untreated control cells. This effect was abolished after 144 hours of VPA treatment. Data are presented as mean + /- standard deviation of three quantitative real-time PCR analyses performed in triplicate. ${ }^{*} 0.01<p \leq 0.05,{ }^{* *} 0.001 \leq p<0.01,{ }^{* * *} p \leq 0.001$

\subsection{Is the Vegfr2 signaling pathway activated by VPA treatment?}

Quantitative real-time PCR analysis of VPA-treated 2E cells revealed an increased expression of the two receptor tyrosine kinases Flt-1/Vegfr1 and Kdr/Vegfr2 as compared to control-treated cells (Fig. 3.5). In order to find out whether the increased expression is correlated to an increased activation of the VEGFR signaling pathway the phosphorylation status of one of the two receptors, namely pKDR/pVEGFR2 was analyzed on protein level by western blot analysis (Fig.3.7), which was then subject to densitometrical evaluation (Fig.3.7, the values are indicated underneath the western blot). Densitometrical evaluation of the western blot revealed an increased pKDR/pVEGFR2 expression in VPA-treated $2 E$ cells as compared to controls. This effect was weak in cells that were treated for 72 hours with VPA but more pronounced in cells that were treated for 144 hours with VPA. This indicates that the increased Kdr/Vegfr2 expression in VPA-treated 2E cells is accompanied with an increased activation of the VEGFR2 signaling pathway as seen by increased pKDR/pVEGFR2 expression in the same cells.

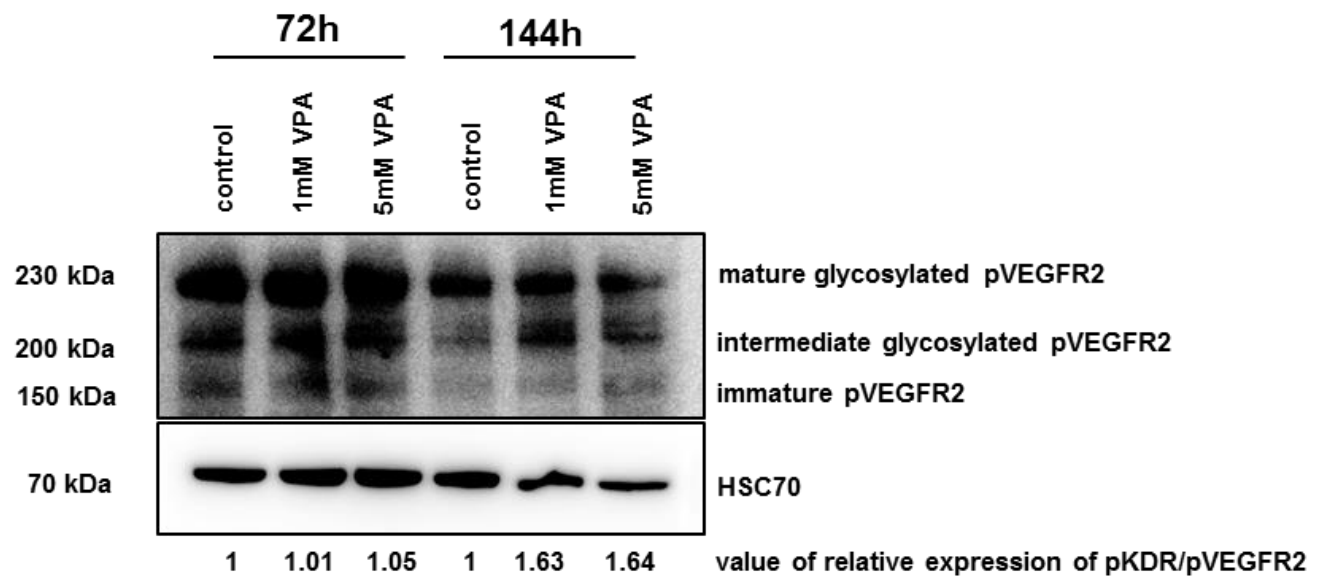


Fig. 3.7: pKDR/VEGFR2 levels in VPA-treated 2E cells. Whole protein lysate of $2 E$ cells treated with either $1 \mathrm{mM}$ or $5 \mathrm{mM}$ VPA for 72 or 144 was immunoblotted using a pKDR/pVEGFR2-specific antibody. Protein lysate of untreated cells served as controls. Immunoblotting of $\mathrm{HSC70}$ ensured equal protein loading. The western blot was evaluated densitometrically and the values are depicted underneath the western blot. Densitometrical analysis revealed an increase in pKDR/pVEGFR2 expression in VPA-treated 2E cells as compared to control-treated cells. This effect was more pronounced in cells that were treated for 144 hours with VPA then in cells that were treated for only 72 hours with VPA.

\subsubsection{Effect of in vivo VPA treatment on the expression of blood- and lymph- angiogenesis markers}

The expression of the six angiogenesis markers, namely Vegfa, Ang1, Tie-1, Flt-1/Vegfr1, Kdrl Vegfr2 and Pecam-1 was analyzed by quantitative real-time PCR on RNA isolated from the prostate of mice that received 10 weeks of in vivo VPA treatment. Only the expression of Flt$1 /$ Vegfr1 was statistically significant downregulated in the prostate of VPA-treated mice as compared to control-treated mice (Fig.3.8). All other factors, namely Ang1, Tie-1, Kdrl Vegfr2 and Pecam-1 showed a reduced expression after VPA treatment in the mouse prostate, but this effect was not statistically significant. Vegfa was not differentially expressed in the prostate of VPA-treated mice as compared to control-treated mice (Fig.3.8). 


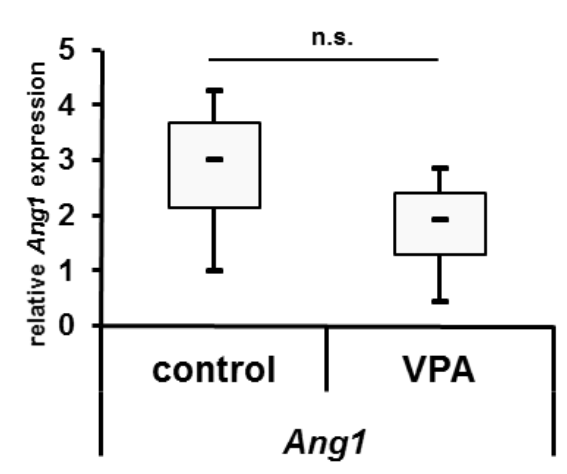

n.s.
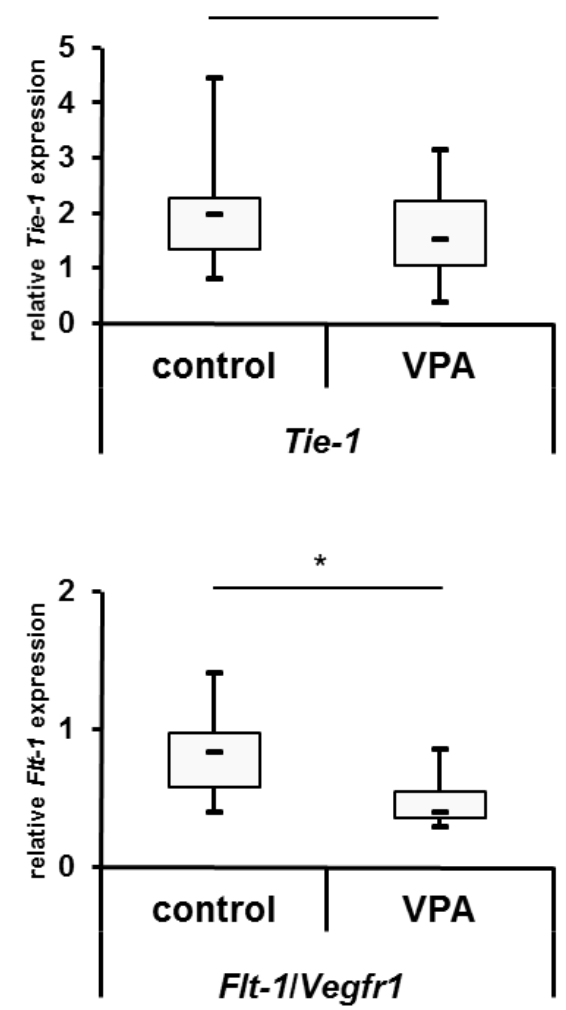
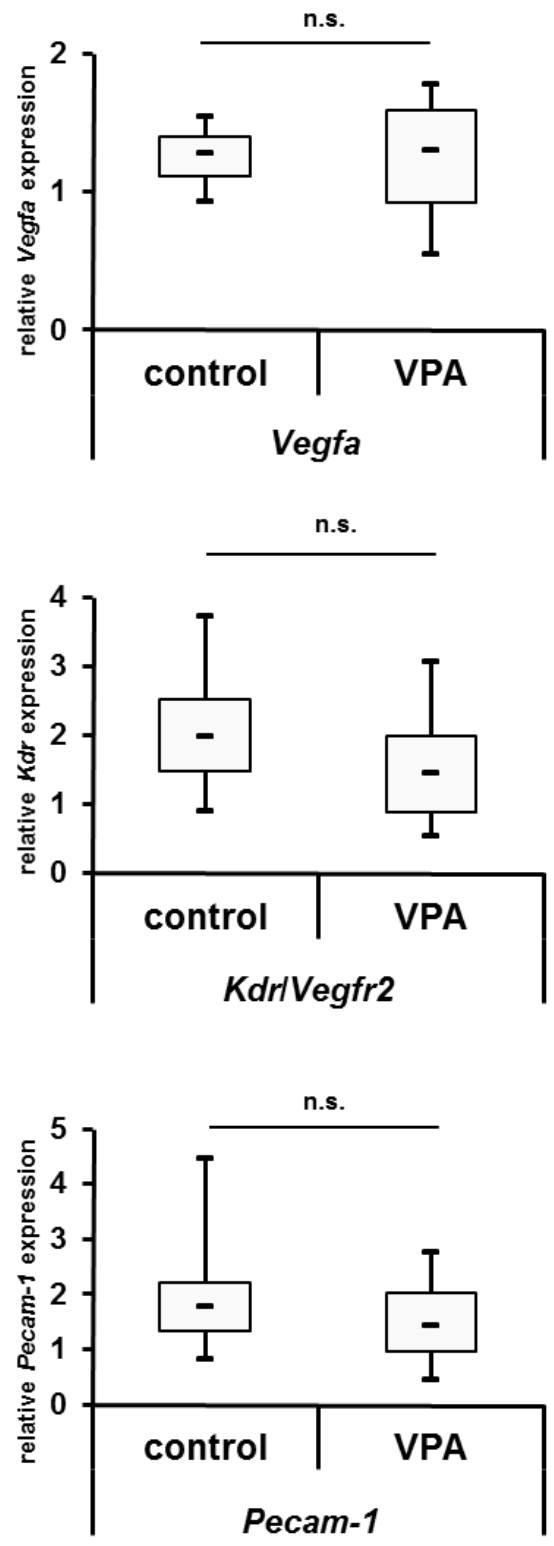

Fig. 3.8: Expression analyses of angiogenesis markers in prostate tissue of in vivo VPA-treated TRAMP mice. RNA was isolated from prostate tissue of in vivo VPA-treated TRAMP mice and of control-treated mice. RNA was reverse transcribed into CDNA and quantitative real-time PCR analysis for expression of the angiogenesis markers Vegfa, Ang1, Tie-1, Flt-1/Vegfr1, KdrlVegfr2 and Pecam-1 was conducted. VPA treatment led to a statistically significant reduction of Flt-1/Vegfr1 expression ( $\mathrm{p}=0.0409$ ). Ang1, Tie-1, Kdr/Vegfr2 and Pecam-1 also exhibited a reduced expression in the prostate tissue after VPA treatment but this effect was not statistically significant. Vegfa was equally expressed in the prostate of VPA- and control-treated TRAMP mice. Data are presented as mean +/standard deviation of three quantitative real-time PCR analyses performed in triplicate. ${ }^{*} 0.01<p \leq 0.05,{ }^{* *} 0.001 \leq p$ $<0.01,{ }^{* * *} p \leq 0.001$, n.s.: not significant

The expression of the six angiogenesis markers Vegfa, Ang1, Tie-1, Flt-1/Vegfr1, Kdr/Vegfr2 and Pecam-1 was also analyzed by quantitative real-time PCR on RNA isolated from prostate tumor tissue of in vivo VPA-treated TRAMP mice and control-treated TRAMP mice (first in vivo 
VPA experiment conducted by D. Witt, 2012). RNA was reverse transcribed into cDNA before it was subjected to quantitative real-time PCR (Fig. 3.9). In the prostate tumors of VPA-treated TRAMP mice a statistically significant reduction of Ang 1, Vegfa, Tie-1 and Kdr/ Vrgfr2 expression was observed as compared to control-treated mice. Pecam-1 expression was also downregulated after VPA treatment, although not statistically significant. The expression of Flt1/Vegfr1 was similar in prostate tumors of VPA-treated mice and control-treated mice (Fig.3.9). Taken together these results indicate that inhibition of expression of angiogenesis markers by VPA treatment could play a more important role during tumor progression than in the beginning of tumor development.
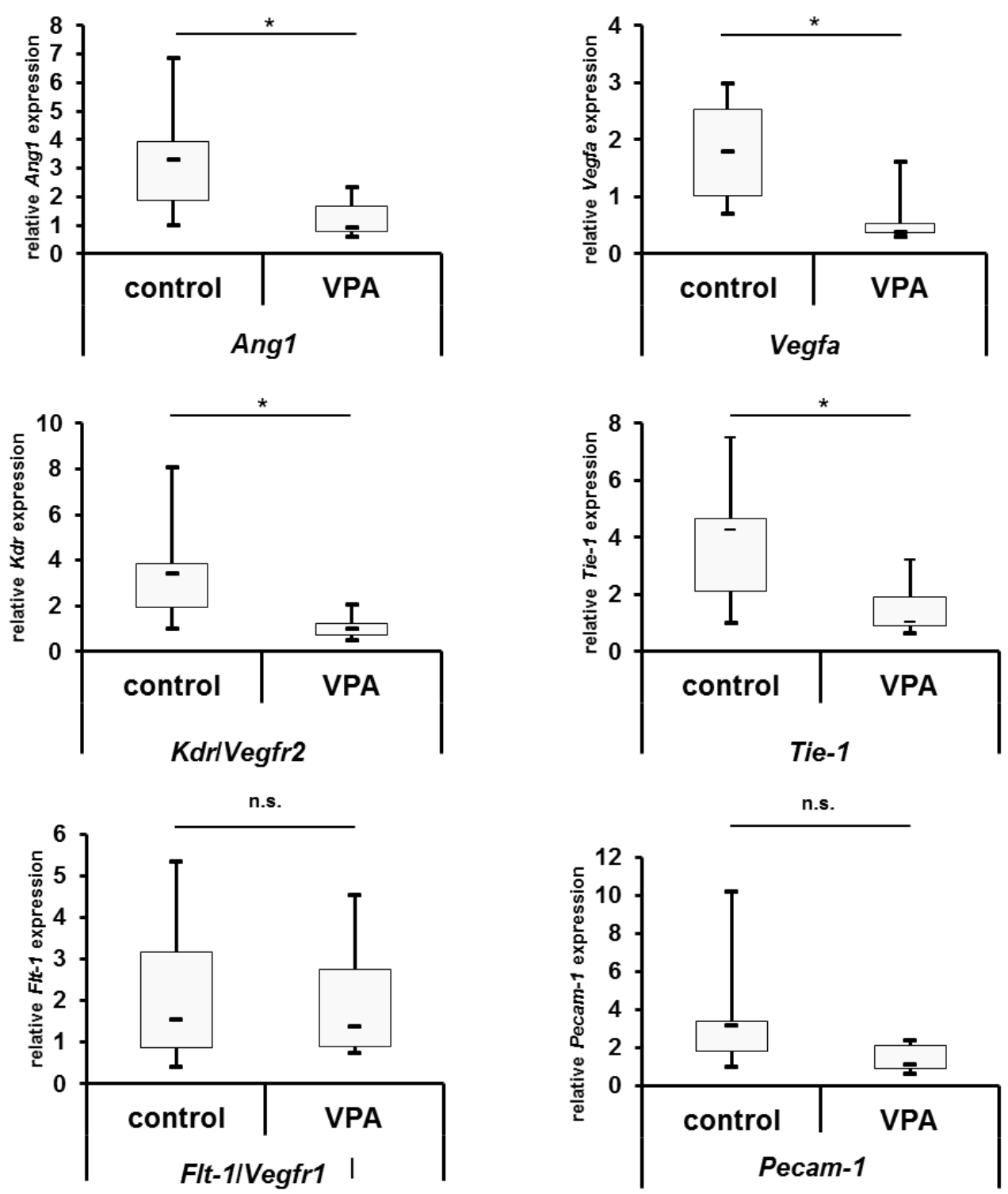

Fig. 3.9: Expression analysis of angiogenesis markers in PCa tumors of in vivo VPA-treated TRAMP mice. RNA was isolated from prostate tumor tissue of in vivo VPA-treated TRAMP mice and of control-treated mice. RNA was reverse transcribed into CDNA and quantitative real-time PCR analyses were conducted for expression analysis 
of the angiogenesis marker Vegfa, Ang1, Tie-1, Flt-1/Vegfr1, KdrlVegfr2, and Pecam-1. VPA treatment led to a statistically significant reduction of Vegfa $(\mathrm{p}=0.0226)$, Ang1 $(\mathrm{p}=0.0427)$, Tie-1 $(\mathrm{p}=0.0462)$ and Kdr/Vegfr2 $(\mathrm{p}=0.0410)$ as compared to prostate tumor tissue of control-treated mice. The expression of Flt-1/Vegfr1 and Pecam-1 was not statistically significant deregulated in prostate tumor tissue of VPA-treated TRAMP mice as compared to prostate tumor tissue of control-treated mice. Data are presented as mean $+/$ - standard deviation of three quantitative realtime PCR analyses performed in triplicate. ${ }^{*} 0.01<p \leq 0.05,{ }^{* *} 0.001 \leq p<0.01,{ }^{* * *} p \leq 0.001$, n.s.: not significant

\subsubsection{Studying the influence of VPA on angiogenesis in vivo - CAM experiments}

Both VPA in vitro and in vivo experiments point to the fact that VPA could have an important influence on angiogenesis during PCa progression. Therefore, to study the effects of VPA treatment on tumor angiogenesis in vivo, the chicken chorioallantoic membrane (CAM) assay was conducted in cooperation with Prof. Dr. Jörg Wilting from the Center of Anatomy, University Medical Center Göttingen. The chicken embryo CAM is an extraembryonic membrane rich of blood vessels and therefore suitable to study the effects of a substance on angiogenesis. Tumor cells applied to the CAM, together with matrigel as a carrier, will form solid tumors which can be harvested and used for molecular evaluation.

For the CAM experiment the mouse PCa cells $2 \mathrm{E}$ and the human PCa cells PC-3 were used. Prior to the application to the CAM the PCa cells were either pre-treated with VPA for 24 hours (experiment 1), for 1 week (experiment 3) or not pre-treated at all (experiment 2). Also different concentrations of VPA were tested (1mM VPA or 3mM VPA, Table 3.1).

Table 3.1: CAM experiments conducted with human and mouse PCa cells. Depicted are the three different CAM experiments conducted during the present study along with the respective PCa cell line and VPA concentration used to treat the cells.

\begin{tabular}{|l|l|}
\hline 1. experiment & 2E and PC-3 cells, 24 hours pre-treatment with 1mM VPA \\
\hline 2. experiment & $\begin{array}{l}\text { 2E and PC-3 cell, no pre-treatment, 3mM VPA on the day of } \\
\text { application to the CAM }\end{array}$ \\
\hline 3. experiment & 2E cells, one week pre-treatment with 3mM VPA \\
\hline
\end{tabular}

Pre-treated or non-pre-treated PCa cells were applied to the CAM and three days thereafter the growing tumor was treated again with VPA in the corresponding concentration. The developed tumors were harvested after one week and fixed or otherwise prepared for further studies, including immunohistochemistry and molecular expression analysis by quantitative real-time PCR. Three different CAM experiments were conducted (Table 3.1). 


\subsection{Macroscopic analysis of the CAM tumors}

The macroscopic analysis revealed three different effects in which the angiogenesis of the CAM tumor could be influenced. First, untreated 2E and PC-3 cells as well as VPA-treated cells could severely disturb angiogenesis as seen by bloodshot tumors (Fig 3.10A). The second effect in which CAM tumor angiogenesis could be affected was observed by bloody spots within the CAM tumor (Fig 3.10B). Finally, the blood vessel formation around the CAM tumor could also be severely affected as seen by disturbed blood vessels (Fig 3.10C).

\section{Unaffected}

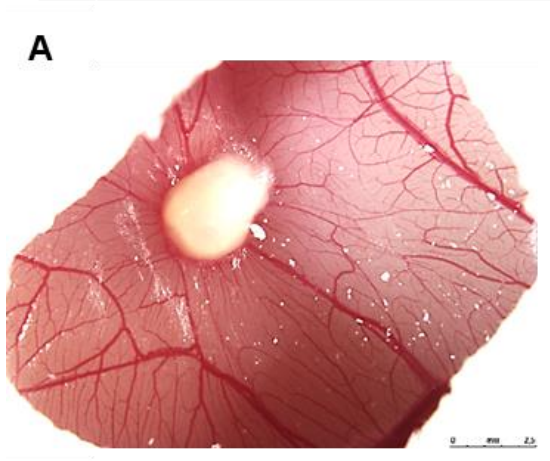

B

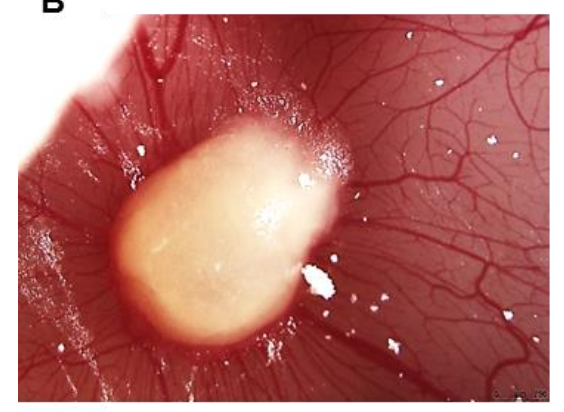

C

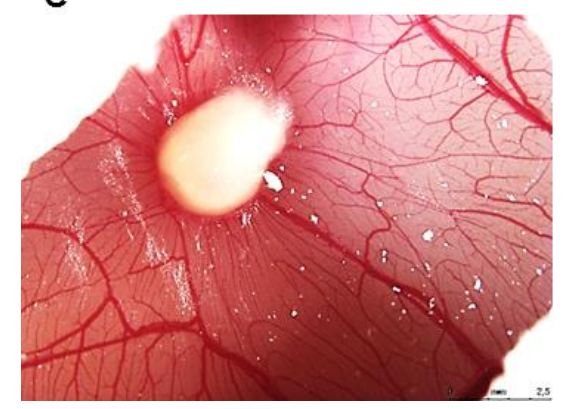

\section{Affected}
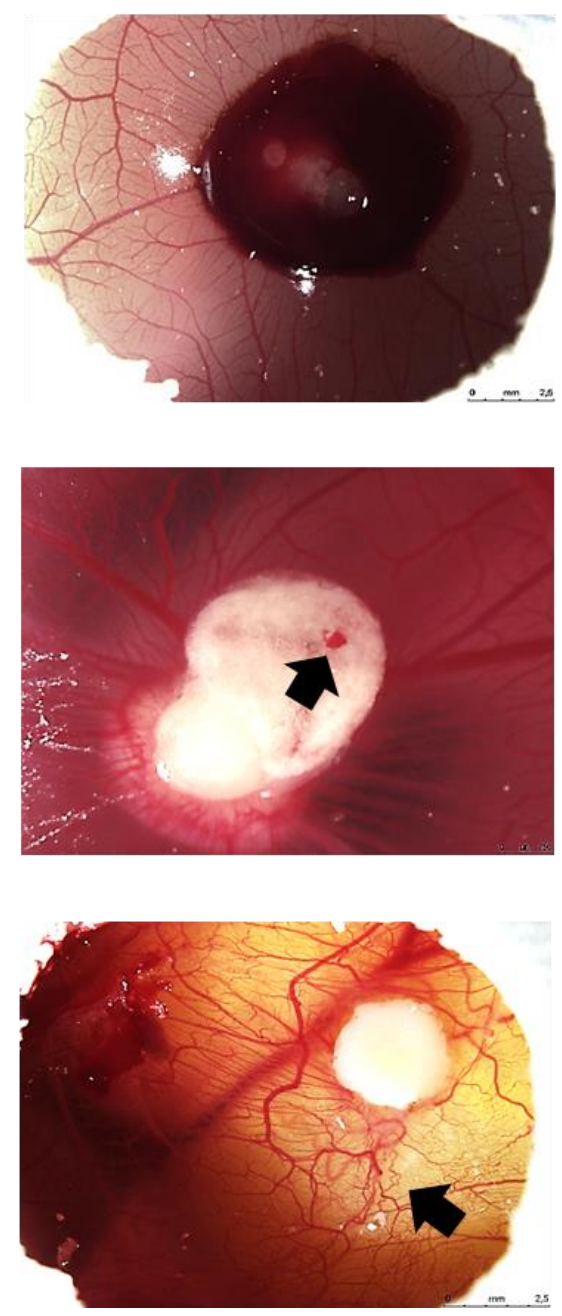

bloodshot tumors

tumors with bloody spots

tumors with disturbed blood vessel formation

Fig. 3.10: Three examples in which CAM angiogenesis can be affected. These exemplary pictures of CAM tumors derived from VPA-treated and untreated control 2E cells were chosen to demonstrate three different effects on CAM tumor angiogenesis compared to an unaffected tumor. The effects were generally observed in all tree CAM experiments for tumors raised from VPA-treated as well as from untreated control cells. A) Tumor angiogenesis could be influenced in that way, that the tumors were severely bloodshot as compared to unaffected tumors. B) Bloody spots within the CAM tumor, as indicated by the arrow, were also observed. C) Affected tumors displayed disturbed 
blood vessel formation around the tumor, indicated by the arrow, as compared to an unaffected tumor. The pictures were taken at an inverted microscope, at a 9x magnification (A), 11x magnification (C) and 19x magnification (B).

For the three CAM experiments the number of bloodshot tumors, tumors with bloody spots and tumors with disturbed vessel formation was calculated for tumors developed from VPA-treated and untreated control cells (Fig. 3.11). In the first experiment tumors resulting from VPA-treated $2 \mathrm{E}$ cells showed bloody spots or were bloodshot, whereas tumors resulting from untreated control 2E cells only showed disturbed blood vessel formation (Fig. 3.11A). In the second experiment, where the cells were not pre-treated with VPA, only with $3 \mathrm{mM}$ VPA on the day of application to the CAM, tumors derived from VPA-treated 2E cells were more often bloodshot than tumors resulting from untreated control 2E cells (Fig 3.11A). This finding is in accordance to the first experiment with VPA-treated 2E cells. In the third experiment, VPA treatment of the $2 \mathrm{E}$ cells resulted in a slightly reduced number of bloodshot tumors, a stronger reduction of tumors with bloody spots, and an increase in tumors with disturbed blood vessel formation (Fig.3.11B). These results from the third experiment are contrary to the observations from the previous two experiments.

Tumors derived from VPA-treated PC-3 cells in the first experiment were not bloodshot at all, whereas tumors resulting from control PC-3 cells were bloodshot (Fig. 3.11B). VPA treatment of the PC-3 cells also resulted in an increased number of tumors with bloody spots as well as with disturbed blood vessel formation compared to tumors resulting from untreated control PC-3 cells (Fig. 3.11B). In the second experiment, tumors resulting from VPA-treated PC-3 cells showed a reduced number of bloodshot tumors compared to tumors derived from untreated control PC-3 cells, as compared to the observation in the first experiment (Fig. 3.11B). Contrary to the first experiment, treatment of PC-3 cells with 3mM VPA resulted in reduced number of tumors with disturbed blood vessel formation as compared to tumors derived from untreated control cell (Fig. 3.11B).

Taken together, a general effect of VPA treatment in PCa cells on the phenotype of CAM tumors could not be observed, since within the experiments for one cell line the phenotypic characteristics did not correlate (except for PC-3 cell tumors, which in both experiments had a reduced number of bloodshot tumors when the cells were treated with VPA) and they also did not correlate within the two cell lines. 


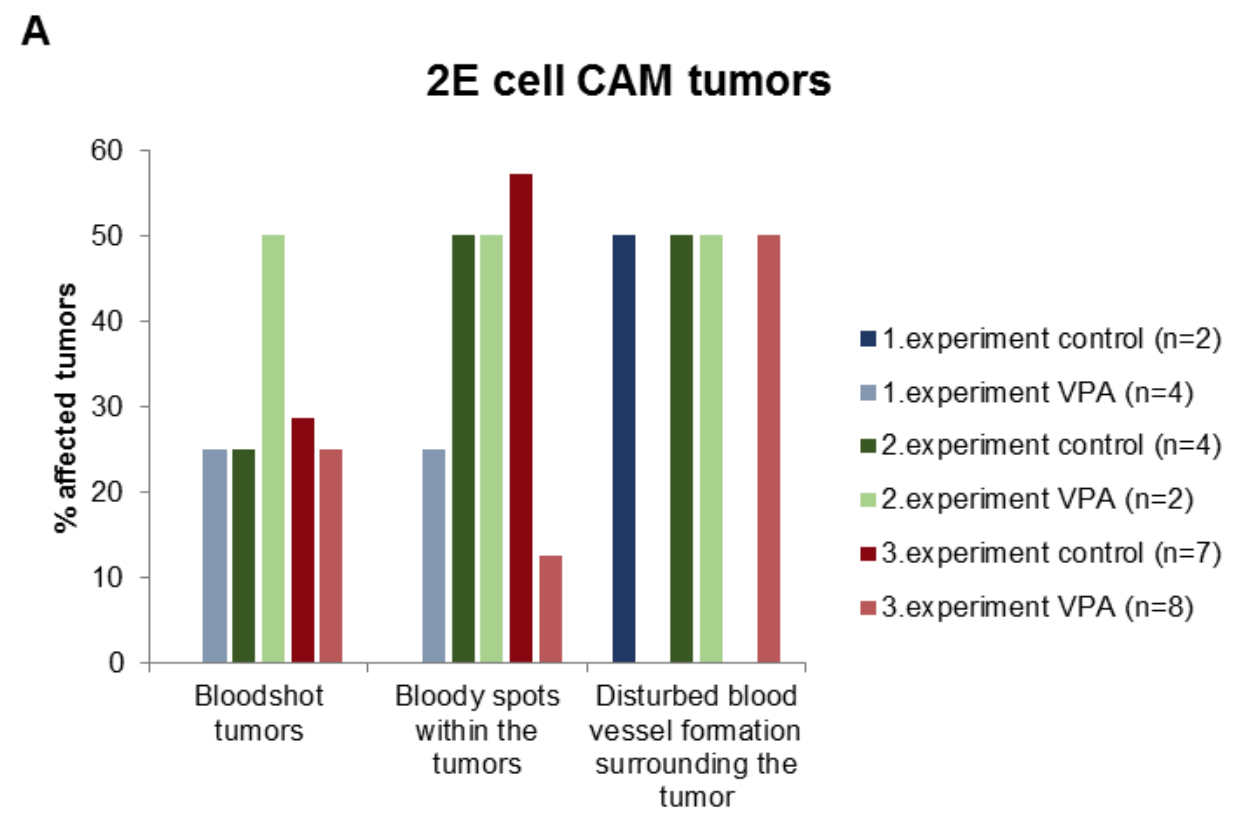

B

\section{PC-3 cell CAM tumors}

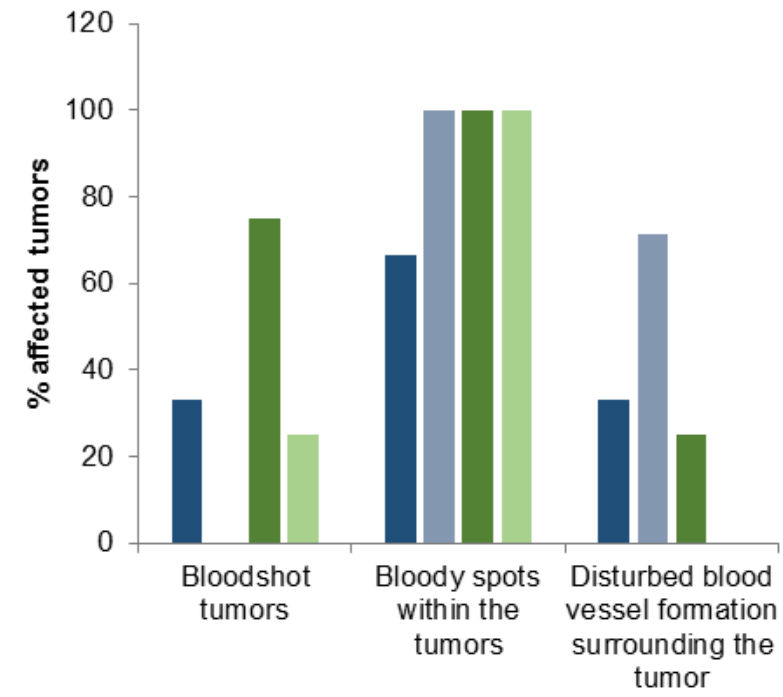

-1.experiment control $(n=3)$

1. experiment VPA $(n=7)$

-2.experiment control $(n=4)$

2. experiment VPA $(n=2)$

Fig. 3.11: Macroscopic analysis of the CAM tumors. For three different CAM experiments the CAM tumors resulting from VPA-treated and control cells were macroscopically analyzed and the number of bloodshot tumors, tumors with bloody spots and tumors with disturbed blood vessel formation was calculated. In the first experiment, tumors resulted from $2 \mathrm{E}$ and $\mathrm{PC}-3$ cells that were pre-treated with $1 \mathrm{mM}$ VPA for 24 hours. In the second experiment, tumors resulted from $2 \mathrm{E}$ and PC-3 cells that were not pre-treated with VPA, only on the day of application to the CAM with 3mM VPA. In the third experiment tumors resulted from 2E cells that were pre-treated with 3mM VPA for 1 week. A) Macroscopic analysis of the CAM tumors of all three CAM experiments conducted with 2E cells. B) Macroscopic analysis of the CAM tumors of the two CAM experiments using PC-3 cells. 


\subsection{Analysis of CAM tumor volume}

Based on the macroscopic pictures the tumor volume was calculated by use of the modified ellipsoid formula 1/2(length $\times$ width $^{2}$ ). 2E cell and PC-3 cell tumors derived from cells that were pre-treated with $1 \mathrm{mM}$ VPA for 24 hours and treated again with $1 \mathrm{mM}$ VPA on the day of application to the CAM showed a slightly reduced but no statistically significant difference in the tumor volume as compared to tumors derived from untreated control cells (Fig. 3.12A). On the other hand, tumors derived from cells that were only treated with 3mM VPA on the day of application to the CAM had a slightly increased tumor volume as compared to tumors derived from untreated control cells, although this effect was also not statistically significant (Fig. 3.12B). $2 \mathrm{E}$ cell tumors derived from cells that were pre-treated with 3mM VPA for one week and again treated with 3mM VPA on the day of application to the CAM had a not statistically significant reduced tumor volume as compared to untreated cells (Fig. 3.12C). These results indicate that only tumors derived from VPA pre-treated PCa cells led to a reduced tumor volume as compared to untreated control cells. PCa cells that were not pre-treated with VPA rather resulted in tumors with an increased tumor volume compared to untreated control cells. Since it is known that VPA treatment has an inhibitory effect on cell proliferation and this could not be confirmed in VPAtreated CAM tumors it appears that the CAM model is not a suitable model for studies with VPA. 


\section{CAM experiment}

A

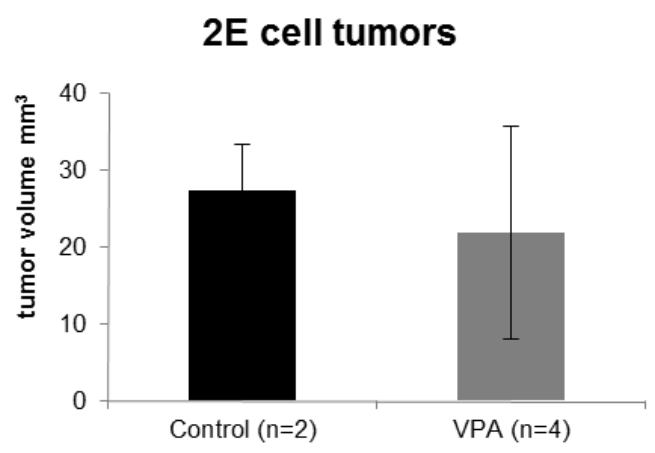

PC-3 cell tumors

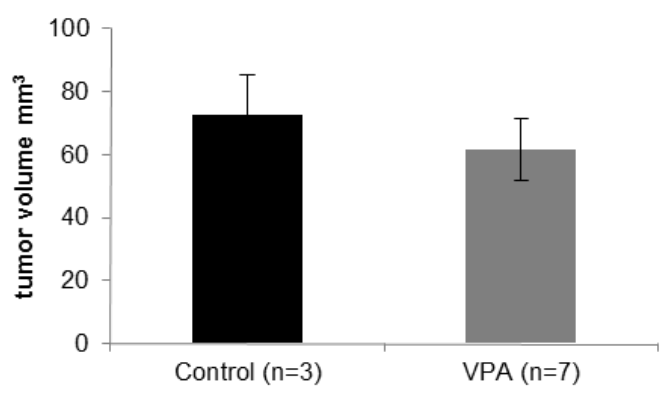

\section{CAM experiment}

B

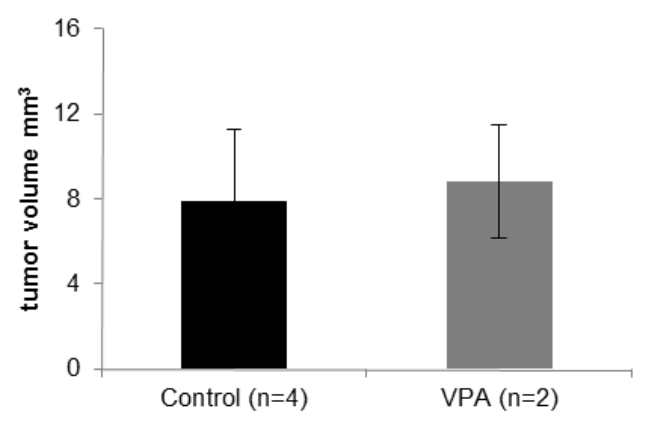

PC-3 cell tumors

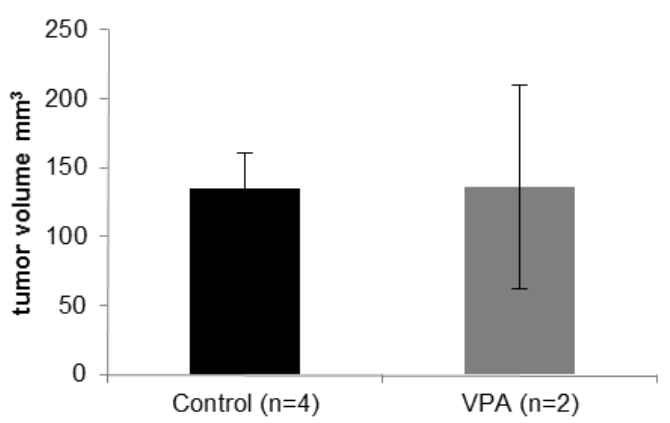

\section{CAM experiment}

C

\section{E cell tumors}

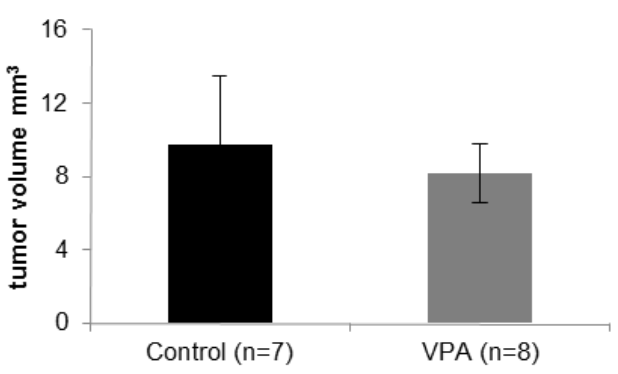

Fig. 3.12: Tumor volume of CAM tumors derived from VPA-treated PCa cells. The tumor volume of CAM tumors derived from VPA- and control-treated 2E and PC-3 cells was calculated by the modified ellipsoid formula based on the macroscopic pictures of the tumors. Tumors derived from untreated PCa cells served as control. Cells that were applied to the CAM were either pre-treated with VPA for 24 hours (A), for 1 week (C) or not pre-treated at all (B). Also different concentrations of VPA were tested (1 mM VPA (A) or 3mM VPA (B, C). A) 2E and PC-3 cell tumors derived from cell that were pretreated with $1 \mathrm{mM}$ VPA for 24 hours and again treated with 1mM VPA on the day of application to the CAM had a not statistically significant reduced tumor volume as compared to tumors derived from untreated control cells. B) 2E and PC-3 cell tumors derived from cells that were treated with 3mM VPA on the day of application 
to the CAM had a slightly increased, also not statistically significant, tumor volume as compared to tumors derived from the control-treated cells. C) 2E cell tumors that were derived from cells that were pre-treated with 3mM VPA for one week and again treated with $3 \mathrm{mM}$ on the day of application to the CAM had reduced, but not statistically significant, tumor volume as compared to tumors derived from untreated control cells.

\subsection{Studying blood and lymphangiogenesis in the CAM tumors with the markers Mep21 and Prox1}

To differentiate the effect of in vivo VPA treatment on lymphatic and blood vessels immunofluorescence staining was performed on 2E and PC-3 cell-derived CAM tumors. Sections of the CAM tumors were stained by immunofluorescence with the blood vessel marker Mep21 (CD34 homolog) and with the lymphatic vessel marker Prox1 (Wigle et al. 2002, Wilting et al. 2002, Hong et al. 2002). The immunofluorescence analysis of tumors grown from untreated mouse 2E and human PC-3 PCa cells revealed that 2E cell tumors had a prominent Mep21 staining and also some Prox1-positive vessels (Fig. 3.13B,C) whereas PC-3 cell tumors had a very prominent staining for Prox1- and Mep21-positive vessels (Fig.3.13F,G). This indicated that tumors of the $2 \mathrm{E}$ cell tumors grow mainly by blood angiogenesis and PC-3 cell tumors by bloodand lymphangiogenesis.

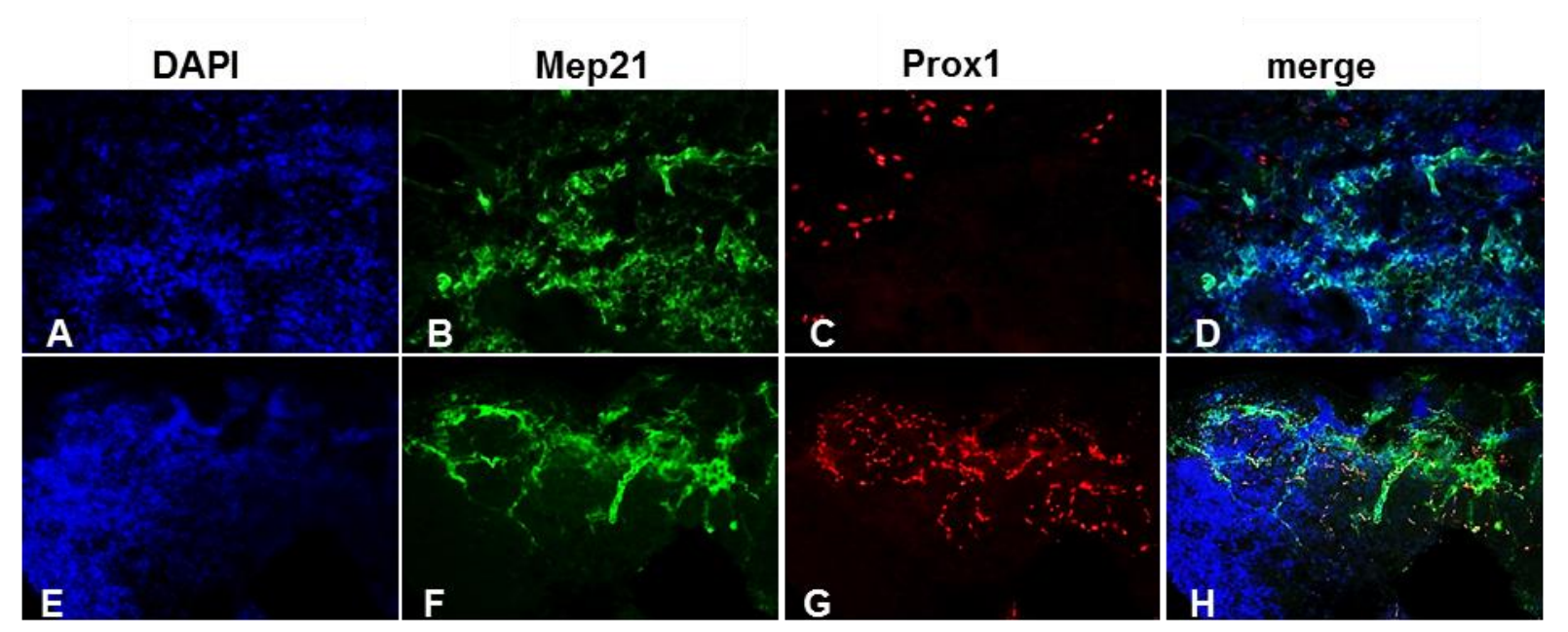

Fig. 3.13: Immunofluorescence staining of blood and lymphatic vessels in untreated 2E and PC-3 tumors. Immunofluorescence staining of cryosections from 2E cell CAM tumors (A-D) and PC-3 cell CAM tumors (E-H). Pictures show staining of blood- and lymphatic vessels in CAM tumors. Cells were stained with DAPI (blue in A, E), anti-Mep21 (green in B, F) and anti-Prox1 (red in C, G). A, E) 2E and PC-3 cells can be discriminated from the chicken cells due to their large nuclei. B, F) Mep21 staining of chicken blood vessel capillaries is very prominent in the 2E and PC-3 cell tumors. C, G) Prox1 staining of chicken lymphatic endothelial cells. PC-3 cell tumors exhibit a very prominent Prox1 staining. D, H) Merged pictures. 
CAM tumor cryo-sections derived from 3mM VPA-treated 2E and PC-3 cells were also subjected to Mep21 and Prox1 immunofluorescent staining. Analysis of VPA-treated 2E cell tumors showed no significant differences in the Mep21 and Prox1 staining as compared to tumors derived from untreated 2E cells (data not shown). Analysis of VPA-treated PC-3 cells tumors led to the impression that they had a reduced number of Prox1-positive cells as compared to tumors derived from untreated PC-3 cells, whereas the Mep21 expression seemed not to be different (data not shown). Since Prox1 stains the nuclei of lymphatic endothelial cells the number of Prox1-positive cells can be calculated. Therefore, the number of Prox1-positive cells in tumors derived from 3mM VPA-treated PC-3 cells und untreated control PC-3 cells was calculated (2. experiment). The tumor was divided in three sections in order to see differences in how far into the tumor lymphangiogenesis had progressed. The first section was above the CAM layer, the second section was the middle part of the tumor and the third part was the distal tumor part (Fig.3.14).
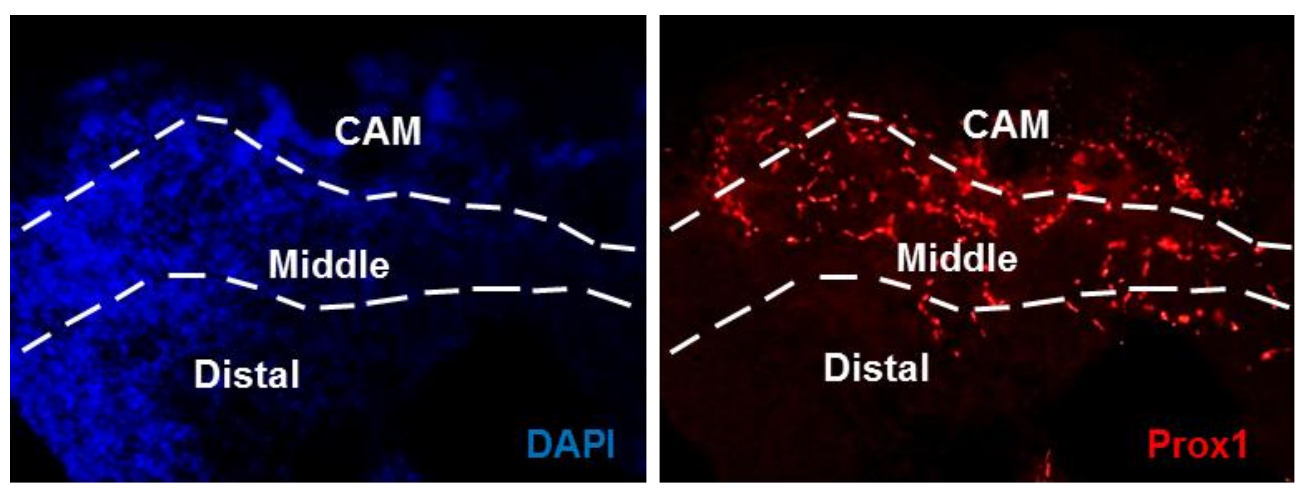

Fig. 3.14: Schematic presentation of the three different sections of the CAM tumor. The CAM tumor was divided in three different sections in order to compare the number of Prox1-positive cells in each of the sections. Prox 1 stains nuclei of chicken lymphatic endothelial cells. The CAM layer was designated as the first section. It can be discriminated from the tumor by the smaller nuclei of chicken cells compared to the bigger tumor cells as seen by DAPI staining. The designated second section was the middle part of the tumor. The distal tumor part was the third section, which contained the least Prox1-positive cells.

Counting of Prox1-positive cells within the tumors did not reveal a statistically significant reduced number of Prox1-positive cells within the tumors of VPA-treated PC-3 cells as compared to tumors raised from control cells. But, in the distal tumor part a reduced number of Prox1-positive cells was observed when the tumor was raised from VPA-treated cells compared to control tumors. Whereas the number of Prox1-positive cells was rather increased in the layer right above the CAM and the middle tumor part (Fig. 3.15). However, increased Prox1 staining in the CAM part and in the middle tumor part is in accordance to the quantitative real-time PCR analysis of the VEGF-system components in untreated and VPA-treated PC-3 cells, where VPA treatment 
resulted in an increased VEGFC expression, which induces mainly lymphangiogenesis by binding to VEGR2 or VEGFR3 (see Fig. 3.6B).

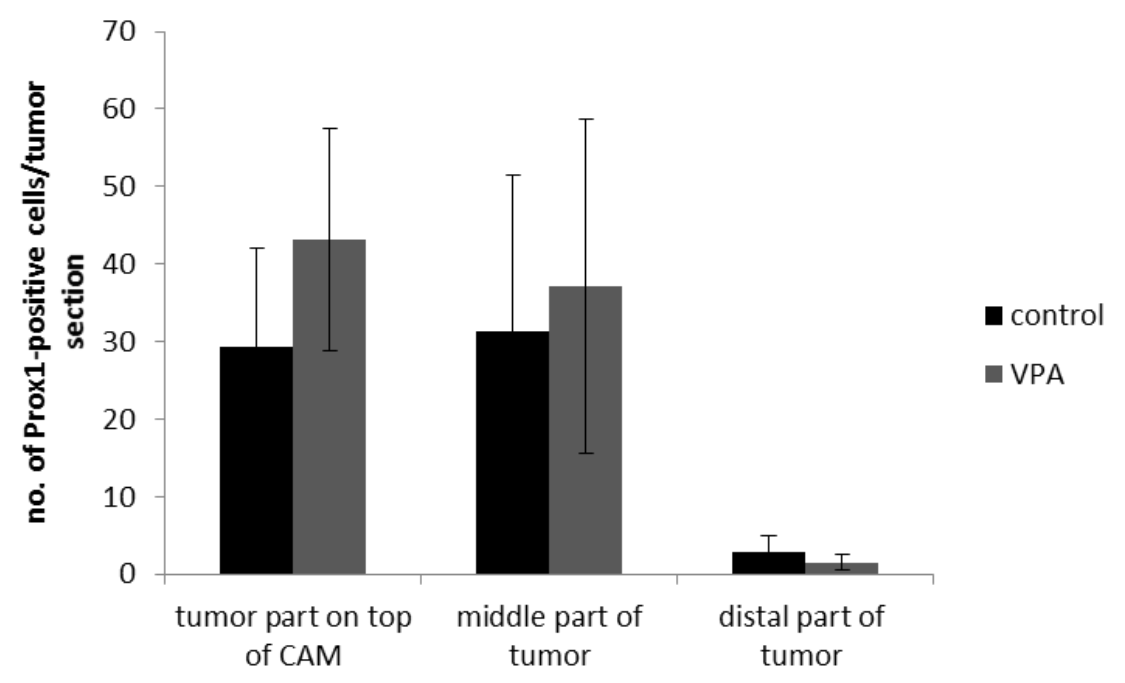

Fig. 3.15: Number of Prox1 positive cells in the CAM tumors. The number of Prox1-positive cells in tumor sections of PC-3 cell CAM tumors was calculated. The number of Prox1-positive cells in tumors derived from 3mM VPA-treated PC-3 cells was compared to the number of Prox1-positive cells in tumors derived from untreated control PC-3 cells. The mean of Prox1-positive cells was calculated for three slides which each consisted of three tumor slices. VPA treatment of the PC-3 cells resulted in an increased number of Prox1-positive cells within the middle part of the tumor and the tumor part right on top of the CAM as compared to Prox1-positive cells in tumors derived from untreated control PC-3 cells whereas in the distal tumor part the number of Prox1-positive cells was reduced, although not statistically significant.

\subsection{Expression analysis of angiogenesis markers in CAM tumors}

RNA was isolated from a whole CAM tumor (1. experiment, 2E and PC-3) or from half of a CAM tumor (3. experiment, 2E). From the second CAM experiment no RNA was isolated. For the PC-3 cell tumors from the first experiment the expression of the members of the VEGFsystem and other angiogenesis markers was analyzed as well as the expression of $C P$ and CCND2. No significant differences in the expression of the VEGF-system members nor CP and CCND2 was observed between tumors grown from untreated control PC-3 cells and VPA-treated PC-3 cells although VPA treatment of the cells seemed to slightly increase the expression of most of the genes tested (Fig. 3.16A). For the 2E cell tumors from the first CAM experiment the expression of the angiogenesis markers Vegfa, Vegfc, Ang1, Tie-1, Flt-1/Vegfr1, Kdr/Vegfr2 and Pecam-1 was analyzed, as well as the expression of the angiogenesis-related candidate genes Cp1, Cp2 and Cxcl15 from the microarray (Witt, 2009) and Ccnd2. The expression of Ccnd2 was examined as a control for the VPA treatment of cells because it is known that VPA treatment leads to a dramatic (about 200x) increase in the Ccnd2 expression in 2E cells. Indeed an 
increase in the Ccnd2 expression in the VPA-treated 2E cell CAM tumors could be observed, although this observation was not statistically significant (Fig. 3.16B). Cp1 expression in VPAtreated CAM tumors was statistically significant downregulated $(p=0.0347)$ and the expression of Vegfa had a strong tendency towards upregulation (Fig. 3.16B). The other tested genes were not significantly deregulated in the tumors of VPA-treated or untreated control 2E cells but their expression was rather increased. The only genes that were downregulated after VPA treatment in 2E cell CAM tumors were $C p 1, C p 2$ and $C x c / 15$, of which solely $C p 1$ was statistically significant. 
A
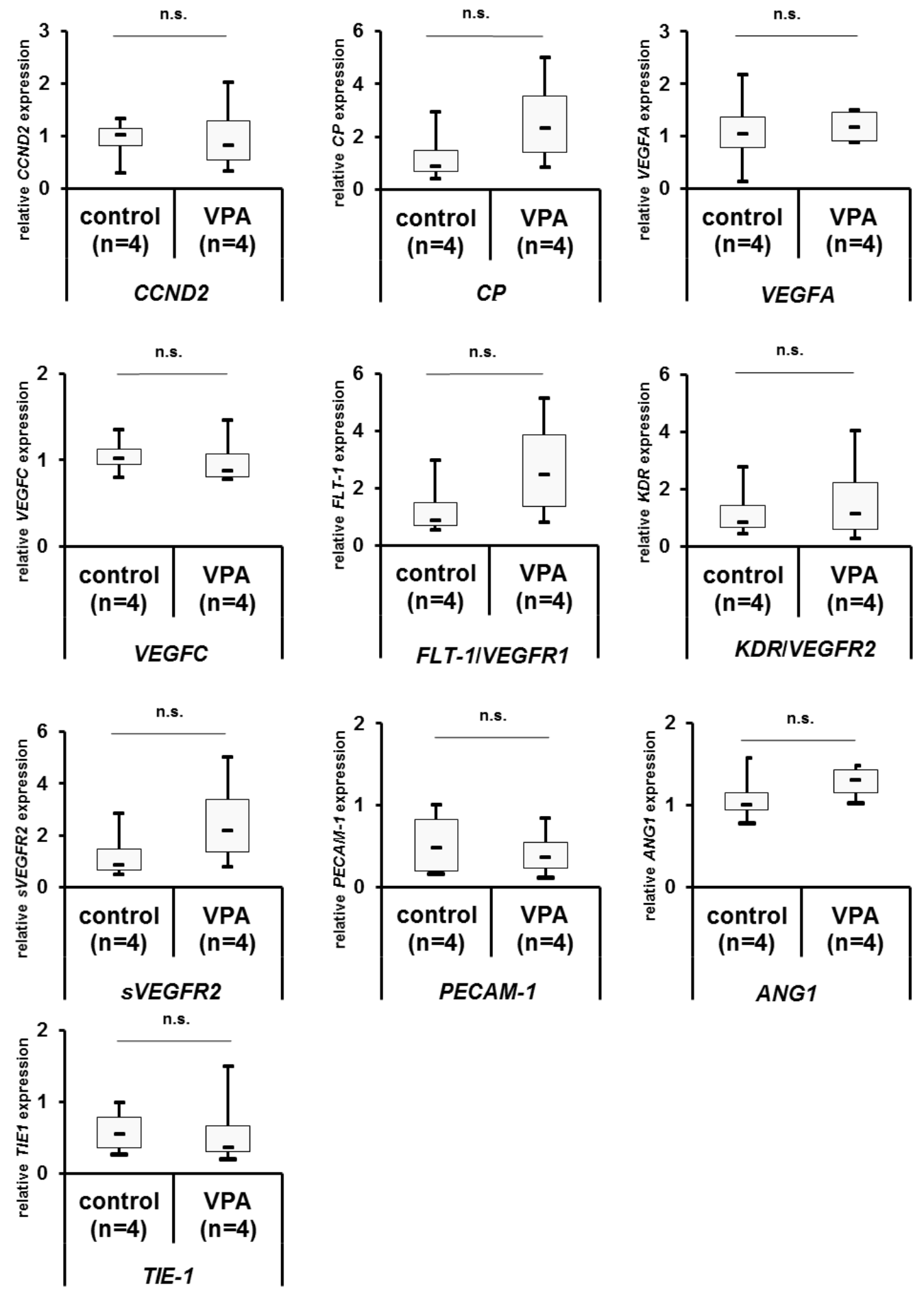
B

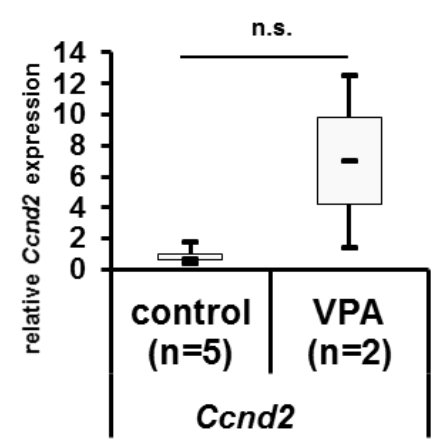

n.s.

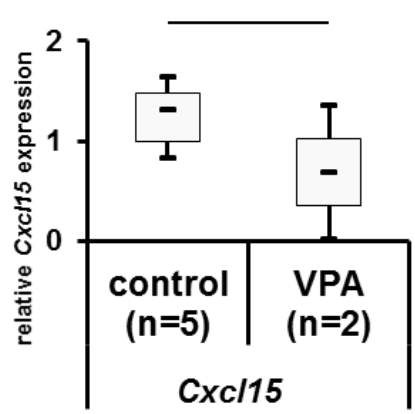

n.s.
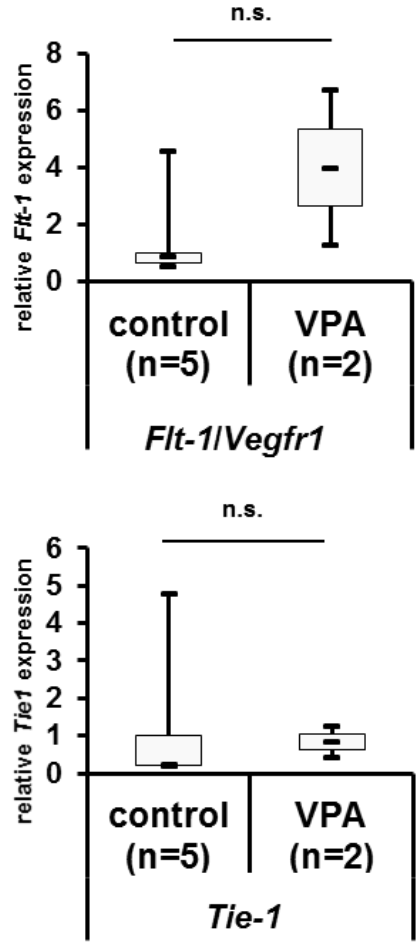

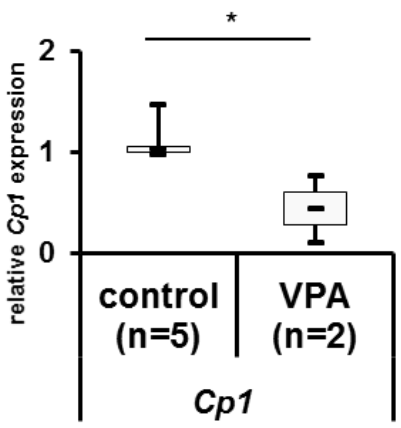

n.s.

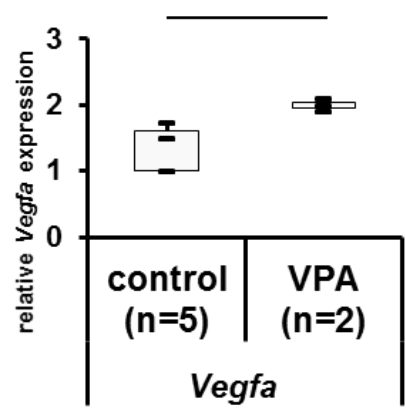

n.s.

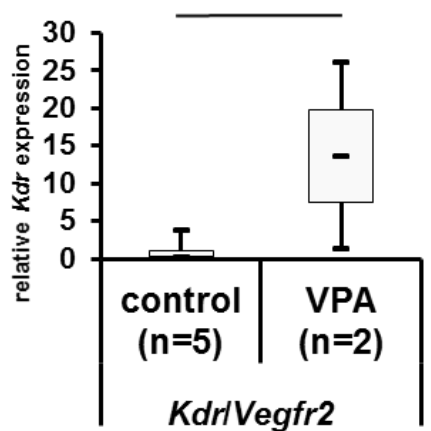

KdrlVegfr2

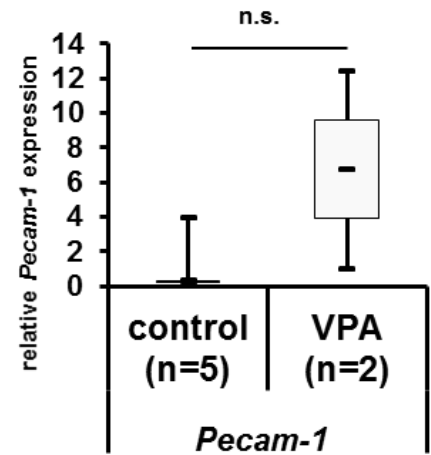

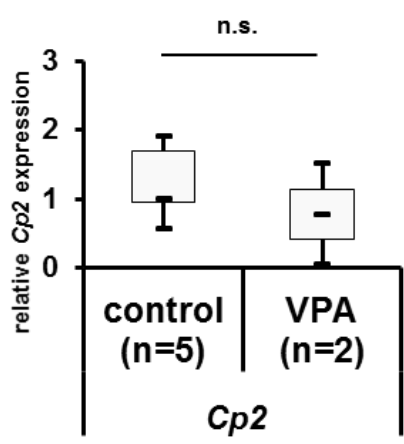
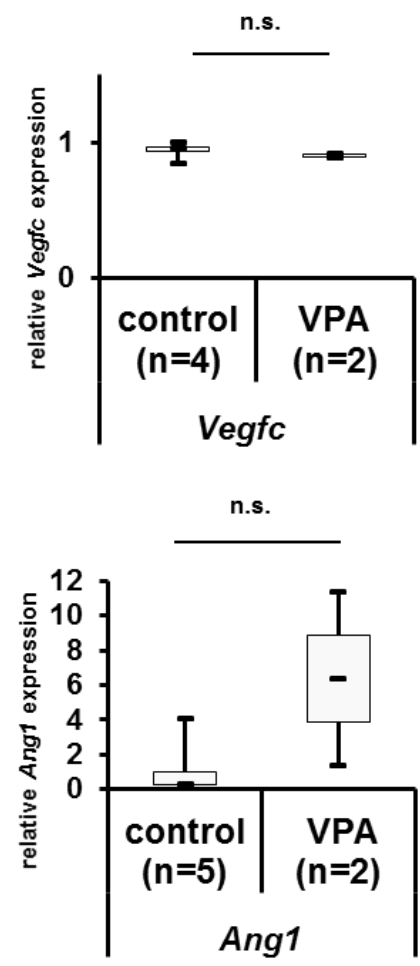

Ang1

Fig. 3.16: Expression analyses of angiogenesis markers and members of the VEGF-system in CAM tumors of the first experiment. The expression of the angiogenesis markers, angiogenesis-related candidate genes from the previous array experiment (Witt, 2009) and of some members of the VEGF-system was analyzed on cDNA generated from CAM tumors that have either developed from VPA-treated PC-3 cells (A) or VPA-treated 2E cells (B) from the first experiment (24 hours pre-treatment with $1 \mathrm{mM}$ VPA). Tumors derived from untreated PCa cells served as control. 
The expression of the following genes was analyzed: VEGFA, ANG1, TIE-1, PECAM-1, FLT-1/VEGFR1, KDR/VEGFR2, sVEGFR2, VEGFC, Cp1 and Cp2 (mouse), CP (human) and CXCL15. The expression of CCND2 was analyzed to show that the VPA treatment was functional because it is known that VPA treatment leads to a major increase in Ccnd2 expression in mouse 2E cells. A) VPA treatment of PC-3 cells resulted in no statistically significant expression differences of the genes analyzed in the CAM tumors as compared to control tumors. B) CAM tumors derived from VPA-treated 2E cells had a statistically significant reduced $C p 1$ expression ( $p=0.0347$ ) as compared to control tumors. The expression of the other genes analyzed was not statistically significant affected after VPA treatment in CAM tumors. Data are presented as mean $+/$ - standard deviation of three quantitative real-time PCR analyses performed in triplicate. ${ }^{*} 0.01<p \leq 0.05,{ }^{* *} 0.001 \leq p<0.01,{ }^{* * *} p \leq 0.001$, n.s.: not significant

The expression of angiogenesis markers and of some members of the VEGF-system was also analyzed for tumors of the third CAM experiment (2E cells only, 1 week pre-treatment with $3 \mathrm{mM}$ VPA, Fig. 3.17). Vegfa was statistically significant upregulated in tumors derived from VPAtreated cells compared to tumors developed from untreated control cells $(p=0.0373)$. The expression of $C c n d 2$ was also significantly upregulated $(p=0.0428)$ which is in accordance to the microarray result of VPA-treated 2E cells, which also had an increased Ccnd2 expression compared to untreated control cells (Witt, 2009). Flt-1/Vegfr 1 expression was not yet statistically significant $(p=0.0874)$. The other genes were not statistically significant deregulated in tumors derived from VPA-treated cells as compared to controls (Fig. 3.17). Generally, the expression of all genes tested was increased after VPA treatment compared to controls in CAM tumors.

Although it was shown in the previous experiments that in vitro and in vivo VPA treatment influences the expression of candidate genes and angiogenesis-related genes this could not be observed and confirmed in studies using the CAM model which further indicates that maybe the CAM model is not the appropriate model for VPA studies. 

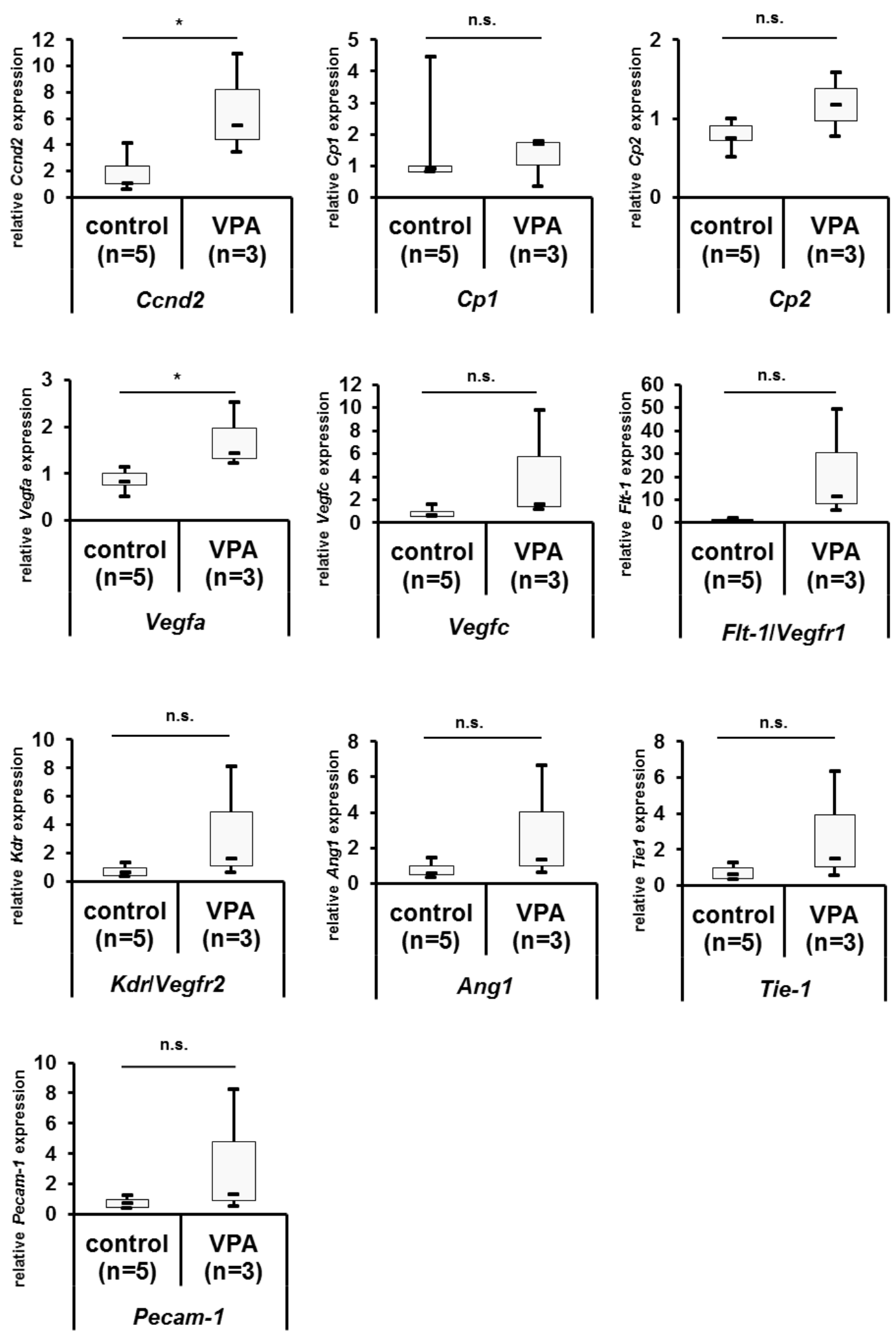

Fig. 3.17: Expression analyses of angiogenesis markers and members of the VEGF-system in CAM tumors of the third experiment. The expression of angiogenesis markers, angiogenesis-related candidate genes from the array (Witt, 2009) and of some members of the VEGF-system was analyzed on cDNA generated from CAM tumors derived from VPA-treated or control-treated 2E cells from the 3. experiment (1 week pre-treatment with 3mM VPA). The 
expression of the following genes was analyzed: Vegfa, Ang1, Tie-1, Pecam-1, Flt-1/Vegfr1, Kdr/Vegfr2, Vegfc, Cp1 and $C p 2$. The expression of $C c n d 2$ was analyzed to show that the VPA treatment was functional because it is known that VPA treatment leads to a major increase in Ccnd2 expression. CAM tumors risen from VPA-treated 2E cells had a statistically significant upregulated expression of Vegfa $(p=0.0373)$ and $C c n d 2(p=0.0428)$ as compared to control tumors. The expression of the other genes analyzed was not statistically significantly affected after VPA treatment. Data are presented as mean + - standard deviation of three quantitative real-time PCR analyses performed in triplicate. ${ }^{*} 0.01<p \leq 0.05,{ }^{* *} 0.001 \leq p<0.01,{ }^{* * *} p \leq 0.001$, n.s.: not significant

\subsection{Is cyclin D2 a tumor suppressor gene in PCa?}

\subsubsection{Cyclin D2 overexpression studies in human PCa cells}

In order to verify the hypothesis that cyclin D2 is a tumor suppressor gene in PCa overexpression studies were conducted. Cyclin D2 is known to be downregulated in PCa due to hypermethylation of the promoter region. Witt et al. (2013) could demonstrate a VPA-induced re-expression of $C c n d 2$ due to increased acetylation of the $C c n d 2$ promoter region. Since the re-expression was accompanied with proliferation inhibition, which was only observed in $\mathrm{PCa}$ cells but not in fibroblast cells or other cancer cells, it is of great interest to investigate the role of cyclin D2 in PCa.

Human PCa cell lines, which have an undetectable low basal cyclin D2 expression, were transfected with a cyclin D2 expression vector and the functional effects were analyzed. If cyclin D2 is indeed a tumor suppressor then these human PCa cells that overexpress cyclin D2 should exhibit less features of a cancer cell, e.g. reduced proliferation and migration rates.

\subsubsection{Transient overexpression of cyclin D2 in human PCa cells}

Initially the human PCa cells were transiently transfected with the generated pIRES2-EGFPCCND2 expression vector to investigate whether the transient overexpression of cyclin D2 might be sufficient to induce its supposed anti-tumorous effects as a putative tumor suppressor.

\subsection{Generation of the pIRES2-EGFP-CCND2 expression vector}

An 870 bp fragment of the cyclin D2 open reading frame was amplified from CaCo2 cDNA by touchdown PCR using the primers CCND2-Fw-Xho and CCND2-Rev-Eco. The amplicon was first cloned successfully into the pGEM®-T Easy vector (data not shown). Thereafter the cyclin D2 insert was excised from pGEM®-T Easy vector and ligated to the pIRES2-EGFP vector. Test 
digestion with the restriction enzymes Xhol and EcoRl (Fig 3.18) and subsequent sequencing reaction (data not shown) of the clones 7 and 9 showed the successful integration of the $870 \mathrm{bp}$ cyclin D2 insert into the pIRES2-EGFP vector.

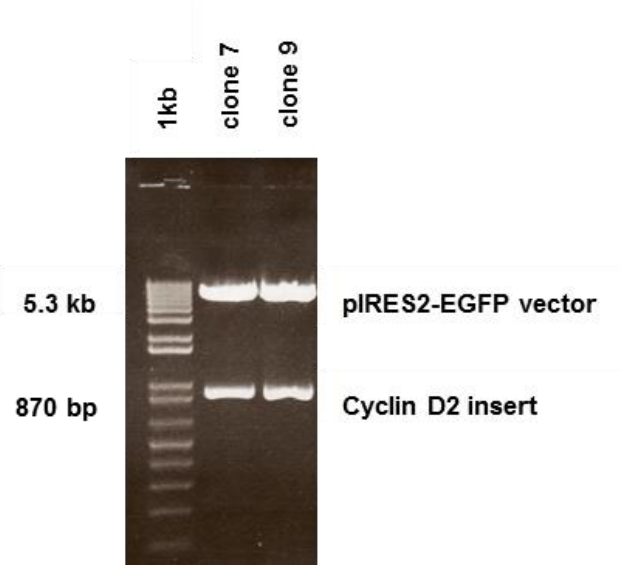

Fig. 3.18: Test digestion of two different pIRES2-EGFP-CCND2 clones. The pIRES2-EGFP-CCND2 clones were digested with the restriction enzymes Xhol and EcoRI. The cyclin D2 insert consisting of 870 bp could be successfully excised from the $5.3 \mathrm{~kb}$ pIRES2-EGFP vector. $1 \mathrm{~kb}$ : $1 \mathrm{~Kb}$ Plus DNA-ladder

The established human PCa cells DU145, LNCaP and PC-3 were transiently transfected with the generated pIRES2-EGFP-CCND2 expression vector using Metafectene $\AA$ Pro transfection reagent. Two days after transfection protein was isolated from the PCa cells to confirm the overexpression of cyclin D2 by western blot analysis. PCa cells that were transfected with the empty pIRES2-EGFP vector were used as controls. For all three human PCa cell lines a very strong expression of cyclin D2 could be detected after transient transfection with the pIRES2EGFP-CCND2 expression vector. In contrast, the control-transfected PCa cells showed no cyclin D2 expression (Fig. 3.19).

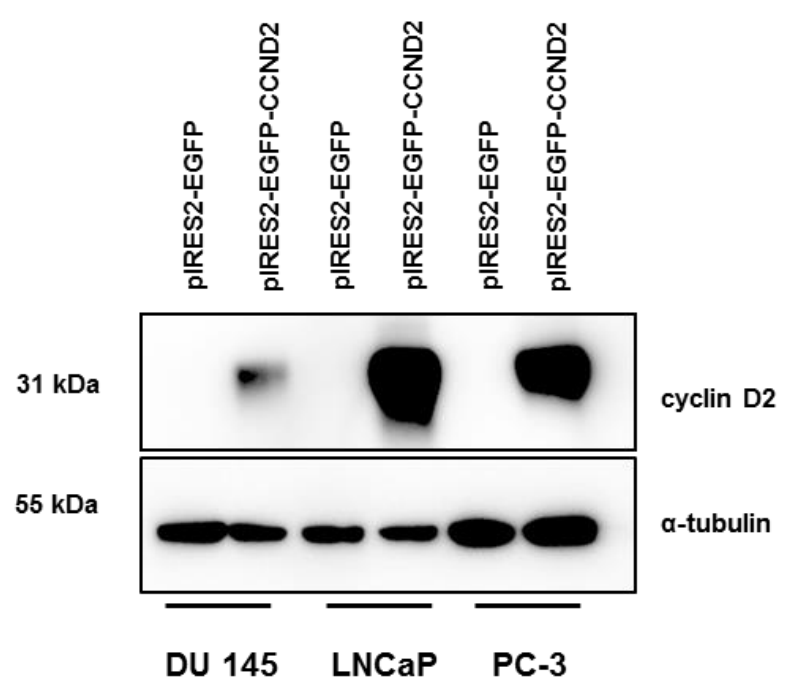


Fig. 3.19: Confirmation of the overexpression of cyclin $D 2$ in human $P C a$ cells after transient transfection with pIRES2-EGFP-CCND2. Whole protein lysates of the PCa cells DU145, LNCaP and PC-3 cells transiently transfected with the generated pIRES2-EGFP-CCND2 expression vector were immunoblotted using a cyclin D2-specific antibody. All three cell lines transfected with the empty pIRES2-EGFP expression vector had no cyclin D2 expression in contrast to the strong cyclin D2 expression in PCa cells transfected with the generated pIRES2-EGFP-CCND2 vector. Immunoblotting of $\alpha$-tubulin ensured equal protein loading.

\subsection{Studies on the proliferation rate of PCa cells with transient cyclin D2 over- expression}

The PCa cells DU145, LNCaP and PC-3 were transiently transfected with the pIRES2-EGFPCCND2 expression vector. Cells transfected with the empty pIRES2-EGFP vector served as controls. The following day the transfected cells were plated into 96- well plates and the proliferation rate was measured every 24 hours for 4 days by a MTT assay.

DU145 cells overexpressing cyclin D2 had a statistically significant increase in the proliferation rate 24 hours after transfection as compared to control cells transfected with the empty pIRES2EGFP vector. The increase in proliferation rate was abolished over the next three days and no differences in the proliferation rate between cyclin D2 overexpressing and control cells could be detected (Fig.3.20). LNCaP and PC-3 cells with transient cyclin D2 overexpression also had a similar proliferation rate as compared to control cells (data not shown).

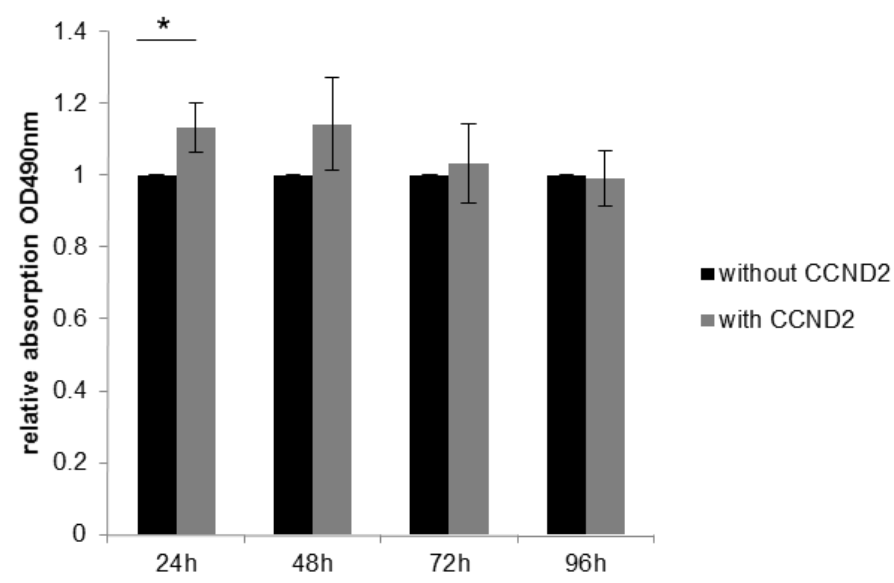

Fig. 3.20: Analysis of the proliferation rate of DU145 cells transiently overexpressing cyclin D2. The human PCa cells DU145 were transiently transfected with the pIRES2-EGFP-CCND2 expression vector. The proliferation rate was measured by a MTT assay every 24 hours for 5 days beginning the day after transfection. After 24 hours there was a statistically significant increase in the proliferation rate of cells transfected with the cyclin D2 expression vector as compared to the control cells transfected with the empty pIRES2-EGFP vector. The increase in the proliferation rate was abolished over the next three days and no differences in the proliferation rate between cyclin D2 overexpressing and control PCa cells could be detected. Data are presented as mean + -- standard deviation of three proliferation assays. ${ }^{*} 0.01<p \leq 0.05,{ }^{* *} 0.001 \leq p<0.01,{ }^{* * *} p \leq 0.001$ 


\subsubsection{Stable overexpression of cyclin D2 in human PCa cells}

Since the transient overexpression of cyclin D2 did not result in any changes or the expected reduction in the proliferation rate as compared to control transfected cells, a different approach was used to verify the hypothesis that cyclin D2 could serve as a tumor suppressor gene in PCa. Presumably a constant overexpression of cyclin D2 is necessary to exhibit its' anti tumorous effects while the transient overexpression of cyclin D2 is not sufficient to induce any functional effects in PCa cells.

Next, PCa cell clones were generated that exhibited a stable overexpression of cyclin D2. For this experiment two different strategies were used, i.e. on the one hand, $\mathrm{PCa}$ cell clones were generated where the overexpression of cyclin D2 was inducible by treatment with doxycycline (transfection with pEBTetD-CCND2) and on the other hand, cell clones were generated which constitutively overexpress cyclin D2 (transfection with pIRES2-EGFP-CCND2).

\subsection{Generation of the pEBTetD-CCND2 expression vector}

An 870 bp fragment of the cyclin D2 ORF was amplified from CaCo2 cDNA by touchdown PCR using the primers CCND2-Fw-Kpn and CCND2-Rev-Eco. The amplicon was first cloned successfully into the pGEM®-T Easy vector (data not shown). Thereafter the cyclin D2 insert was excised from $\mathrm{pGEM} \mathrm{B}_{-T}$ Easy vector and ligated to the $\mathrm{pEBT}$ etD vector. Test digestion with the restriction enzymes Kpnl and Notl (Fig. 3.21) and subsequent sequence analysis (data not shown) of one clone showed the successful integration of the 870 bp cyclin D2 insert into the pEBTetD vector.

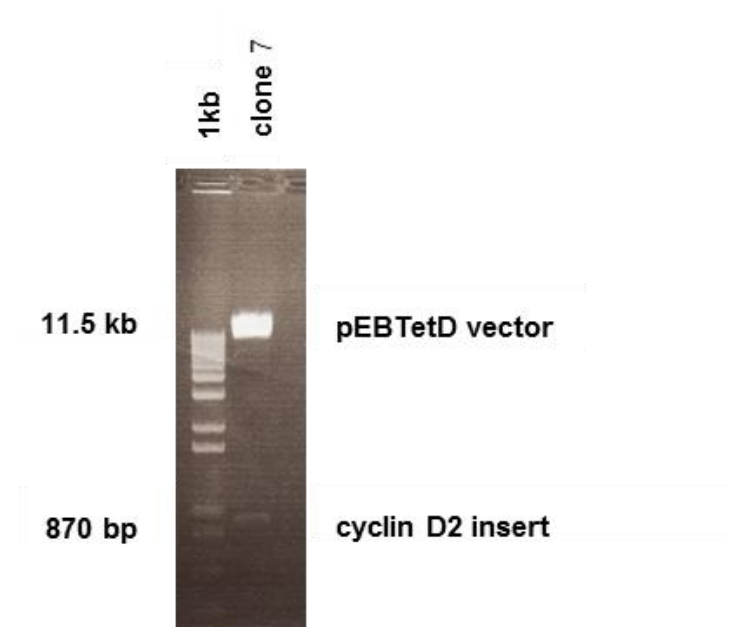

Fig. 3.21: Test digestion of a pEBTetD-CCND2 clone. The pEBTetD-CCND2 clone was digested with the restriction enzymes Kpnl and Notl. The 870 bp cyclin D2 insert could be successfully excised from the $11.5 \mathrm{~kb}$ pEBTetD vector. 1kb: $1 \mathrm{~Kb}$ Plus DNA-ladder 
The established human PCa cells LNCaP and PC-3 were transfected with the generated pEBTetD-CCND2 expression vector using Metafectene® Pro transfection reagent for inducible overexpression of cyclin D2 by treatment with doxycycline. Starting the following day after transfection cells were selected by puromycin treatment. Since the vector does not integrate into the genome but exists as an episomal plasmid within the cytoplasm there was no need for clonal isolation of transfected cells (Bach et al., 2007). Cells that had taken up the pEBTetD-CCND2 expression vector with the puromycin resistance cassette were further cultured in the presence of puromycin. To test for successful uptake of the expression vector and inducibility of cyclin D2 expression protein was isolated and analyzed by western blot analysis. For this approach two different LNCaP cell populations with a doxycycline-inducible expression of cyclin D2 could be established (LNCaP+pEBTetD-CCND2-1 and LNCaP+pEBTetD-CCND2-2) as well as one LNCaP cell population that was transfected with the empty pEBTetD plasmid (LNCaP+pEBTetD) which served as a negative control (Fig. 3.22). There is a strong induction of cyclin D2 expression 72 hours after the two LNCaP cell populations were treated with different concentrations of doxycycline. LNCaP cells not treated with doxycycline showed no cyclin D2 expression comparable to LNCaP cells transfected with the empty pEBTetD plasmid after doxycycline treatment. Doxycycline-inducible cyclin D2 overexpressing PC-3 cells could also be generated (Fig. 3.22, PC-3+D2) but after cryoconservation and subsequent repeated testing a loss of the doxycycline-inducible cyclin D2 overexpression had occurred (data not shown). Therefore, only LNCaP cells transfected with pEBTetD-CCND2 could be used for studies on the functional effects of inducible cyclin D2 overexpression.

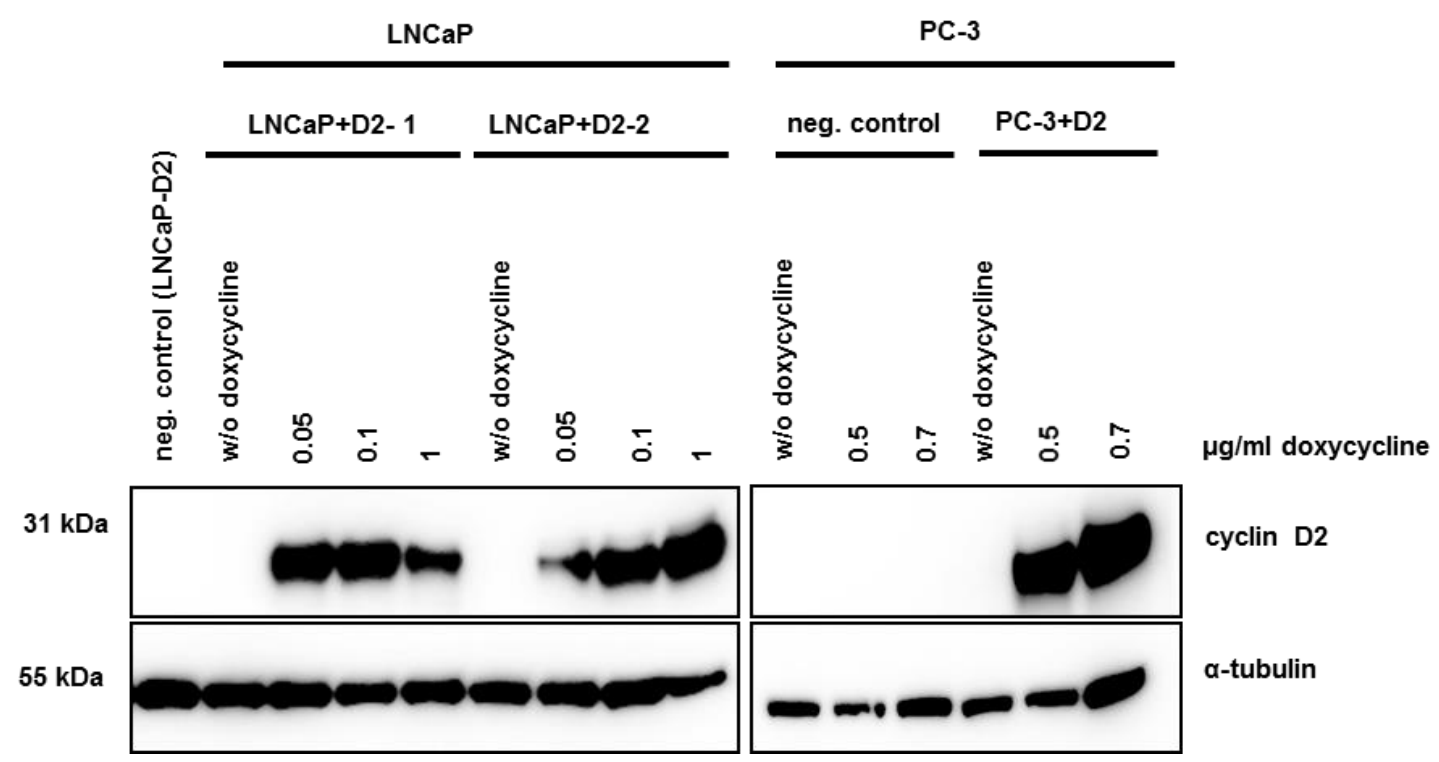

Fig. 3.22: Confirmation of the doxycycline-inducible overexpression of cyclin D2 in human PCa cells after transfection with pEBTetD-CCND2. Whole protein lysates of LNCaP and PC-3 cell populations transfected with the 
generated pEBTetD-CCND2 expression vector and treated for 72 hours with doxycycline were immunoblotted using a cyclin D2-specific antibody. Different concentrations of doxycycline were tested, ranging from $0.05 \mu \mathrm{g} / \mathrm{ml}$ to $1 \mu \mathrm{g} / \mathrm{ml}$ doxycycline. Both LNCaP cell populations transfected with the pEBTetD-CCND2 expression vector as well as the one PC-3 cell population showed a strong induction of cyclin D2 expression after treatment with the indicated concentrations of doxycycline in contrast to the control cells that were not treated with doxycycline. Cells transfected with the empty pEBTetD vector, which served as a second negative control, had also no cyclin D2 expression. Immunoblotting of $\alpha$-tubulin ensured equal protein loading.

The established human PCa cells LNCaP and PC-3 were also transfected with the generated pIRES2-EGFP-CCND2 expression vector (see section 3.2.1.1.1) using Metafectene® Pro transfection reagent for constitutively overexpression of cyclin D2. Two days after transfection the cells were serially diluted and plated into petri dishes do generate single-cell clones. From the next day on selection of transfected cells occurred by treating them with $400 \mu \mathrm{g} / \mathrm{ml} \mathrm{G} 418$. After approximately 21 days single-cell clones were picked and further cultured in the presence of G418. To test for successful stable overexpression of cyclin D2 protein from different cell clones (clones 1-3) including one cell clone transfected with the empty pIRES2-EGFP vector as control (clone 4) was isolated. As seen in Fig. 3.23 the three LNCaP clones exhibit a very strong cyclin D2 expression in contrast to the control clone which exhibits no cyclin D2 expression. For the PC-3 cells only two stable cyclin D2 overexpressing clones could be generated, as seen in Fig. 3.23 by the strong cyclin D2 expression of clone 1 and 3. However, the control clone 4 showed no cyclin D2 expression.

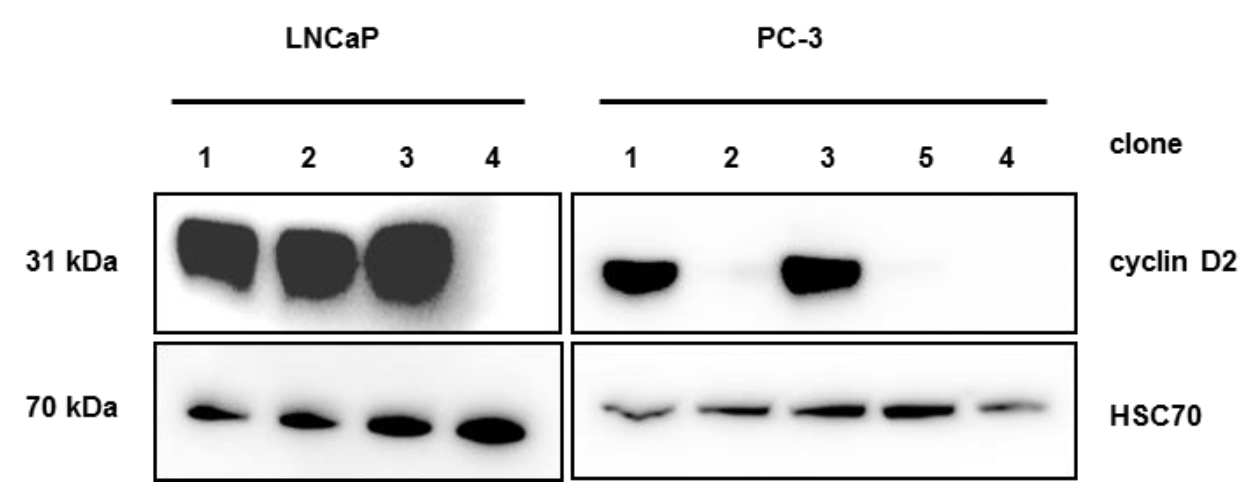

Fig. 3.23: Confirmation of the stable overexpression of cyclin D2 in human PCa cells after transfection with pIRES2-EGFP-CCND2. Whole protein lysates of LNCaP and PC-3 cell clones stably transfected with the pIRES2EGFP-CCND2 expression vector were immunoblotted using a cyclin D2-specific antibody. All three LNCaP cell clones transfected with the pIRES2-EGFP-CCND2 expression vector showed a strong induction of cyclin D2 expression, whereas only two of the four tested PC-3 cell clones transfected with the pIRES2-EGFP-CCND2 expression vector showed a strong induction of cyclin D2 expression (clone 1 and 3) in contrast to the control clones transfected with the empty pIRES2-EGFP vector (clone 4, respectively). Immunoblotting of HSC70 ensured equal protein loading. 
When cells transfected with the pIRES2-EGFP or pIRES2-EGFP-CCND2 vector indeed incorporated the plasmid DNA then these cells should be green fluorescent because of the EGFP gene transcription. The presence of green fluorescent cells was reviewed under the inverted fluorescence microscope. All three generated LNCaP cell clones with a stable cyclin D2 overexpression (clone 1-3) exhibited about 100\% green fluorescent cells (Fig. 3.24A). Cells of the control transfected clone (4) without the cyclin D2 construct were also green fluorescent (Fig. 3.24A). Equally, the two generated PC-3 cell clones stably overexpressing cyclin D2 consisted only of green fluorescent cells as well as the cells of the control transfected clone (4) without the cyclin D2 construct (Fig. 3.24B).

A

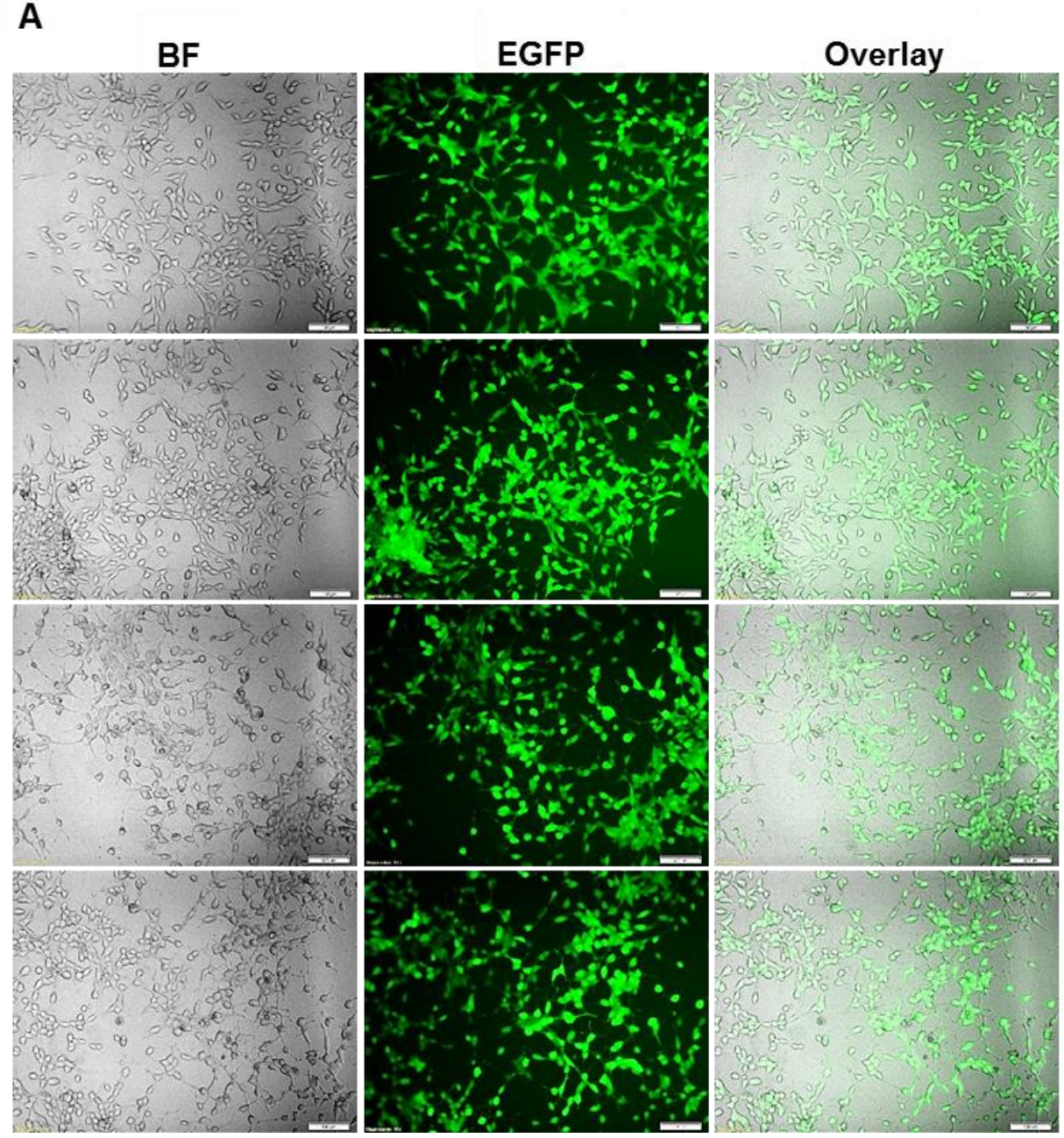

LNCaP

clone 1

LNCaP

clone 2

LNCaP

clone 3

LNCaP

clone 4

(control) 


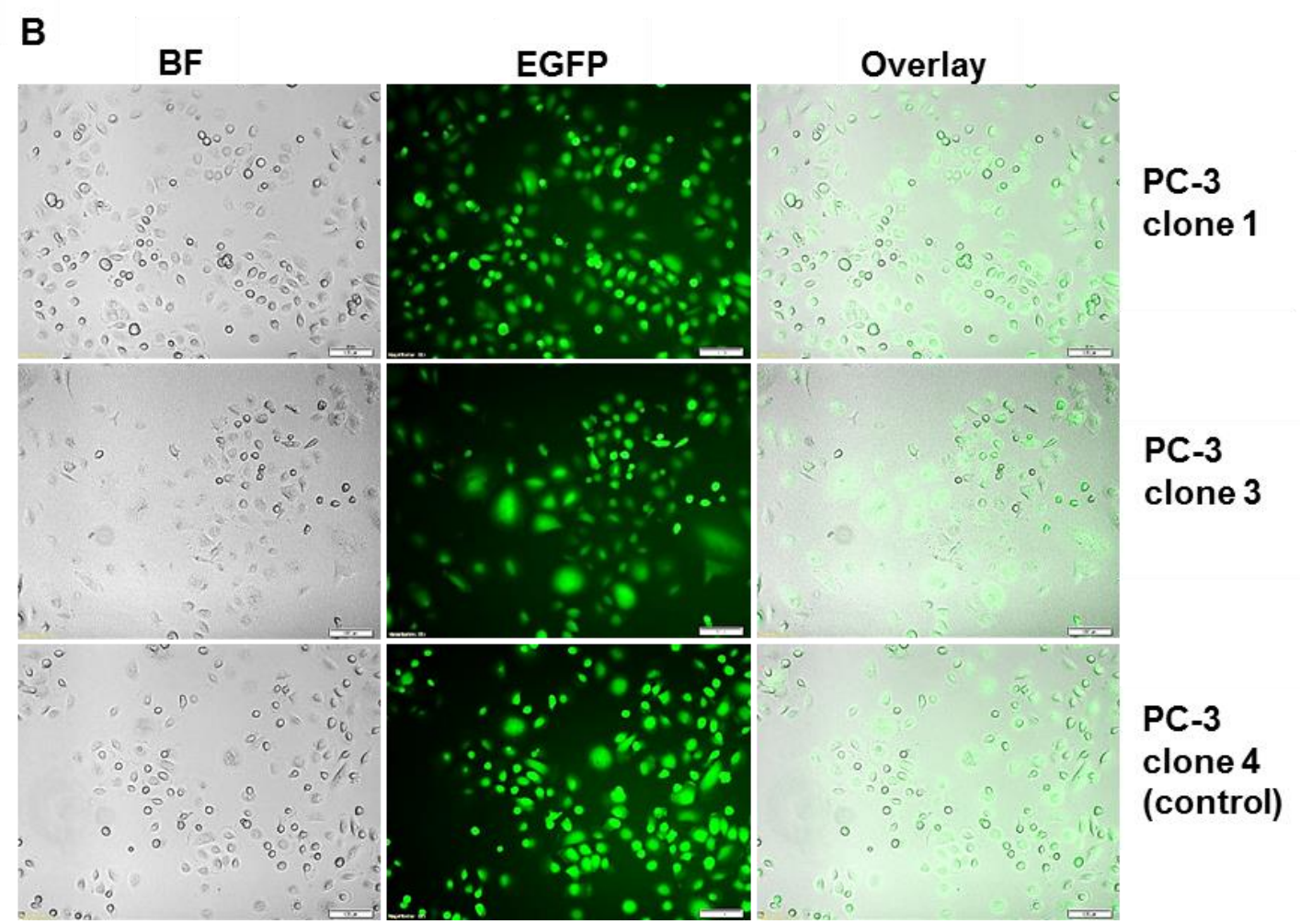

Fig. 3.24: Verification of the presence of green fluorescent cells after transfection with pIES2-EGFP-CCND2. The presence of the incorporated EGFP construct from the pIRES2-EGFP-CCND2 plasmid DNA in the human PCa cells $\mathrm{LNCaP}$ (A) and PC-3 (B) was verified by the presence of green fluorescent cells. For both cell lines the generated clones consisted to about $100 \%$ of green fluorescent cells. Pictures were taken on the inverse microscope at a 10x magnification.

Another method used to show that the generated PCa-pIRES2-EGFP-CCND2 cell clones indeed exhibit cyclin D2 overexpression was by immunofluorescence staining. Therefore, cells were plated on glass culture slides, fixed with 3.7\% formaldehyde after 24 hours and immunostained using a cyclin D2-specific antibody. Cells of clone 4 which were transfected with the empty pIRES2-EGFP plasmid served as controls. About $100 \%$ of the cells of the different clones stained positive for cyclin D2. Cells of two control clones (clone 4, respectively) showed no cyclin D2 overexpression (Fig. 3.25). 


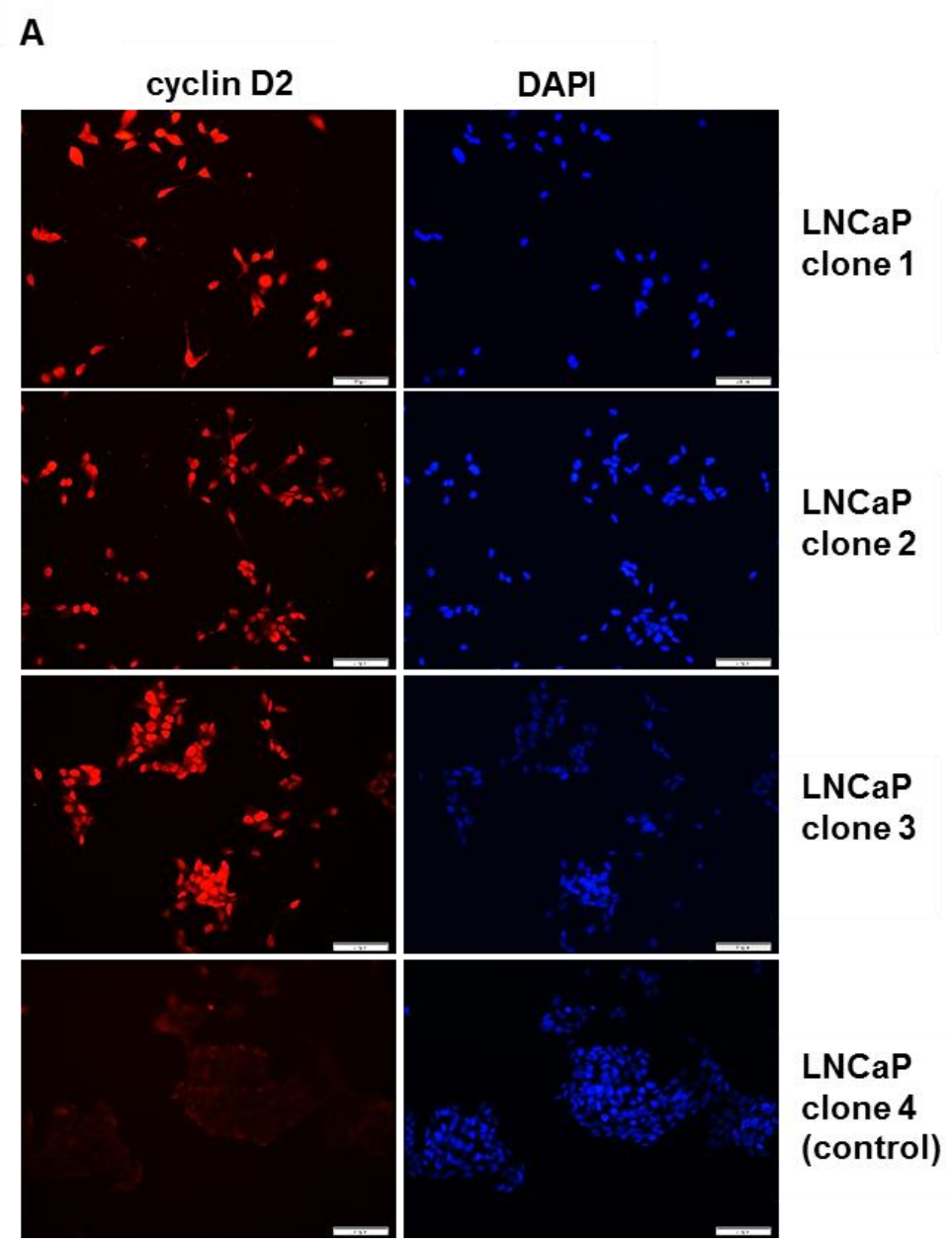

B

cyclin D2

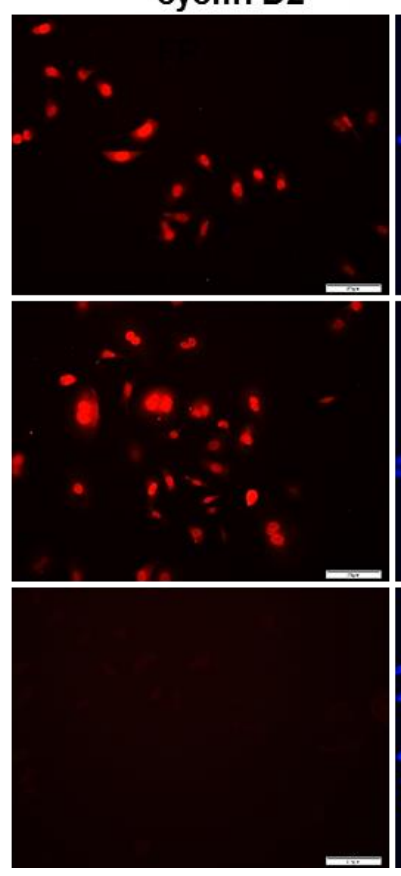

DAPI

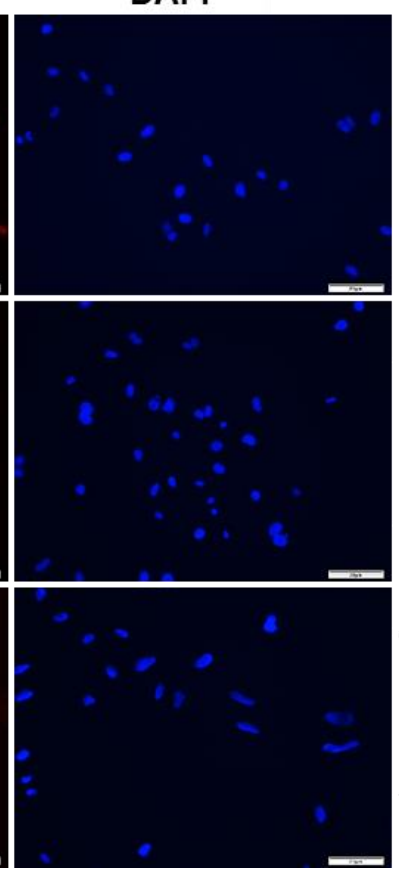

PC-3

clone 1

PC-3

clone 3

PC-3

clone 4

(control) 
Fig. 3.25: Verification of cyclin D2 overexpression in the generated PCa cell clones by immunofluorescence staining. The generated cyclin D2 overexpressing LNCaP (A) and PC-3 (B) clones were tested by immunofluorescence staining for their cyclin D2 expression. The cells were fixed and stained by the use of a cyclin D2-specific antibody (anti-cyclin D2 (M-20)). Nuclei were stained with DAPI. About 100\% of the cells stained positive for cyclin D2. Cells of control clones which were transfected with the empty pIRES2-EGFP plasmid showed no cyclin D2 overexpression (clone 4 , respectively). The pictures were taken on a fluorescence microscope at a $20 x$ magnification.

\subsection{Functional effects of stable and inducible cyclin D2 overexpression}

\subsection{Studies on the proliferation rate of PCa cells with stable or inducible cyclin D2 overexpression}

The proliferation rate of the cell populations with doxycycline-inducible cyclin D2 overexpression and of the cell clones with stable cyclin D2 overexpression was measured every 24 hours for 4 days by MTT assay. Cells of the two LNCaP+pEBTetD-CCND2 populations, which were treated with doxycycline $(0.025 \mu \mathrm{g} / \mathrm{ml})$ to induce the cyclin D2 overexpression, showed a statistically significant increase in the proliferation rate as compared to $\mathrm{LNCaP}$ control cells transfected with the empty pEBTetD plasmid (Fig. 26A).

All three LNCaP clones stably overexpressing cyclin D2 showed an increase in the proliferation rate as compared to the LNCaP control clone which did not show cyclin D2 overexpression (Fig. 3.26B). The same result was observed for the two PC-3 clones overexpressing cyclin D2 which also exhibit a slight, but not statistically significant, increased proliferation rate compared to the PC-3 control clone (Fig. 26C). 
A

LNCaP+pEBTetD-CCND2, $0.025 \mu \mathrm{g} / \mu \mathrm{l}$

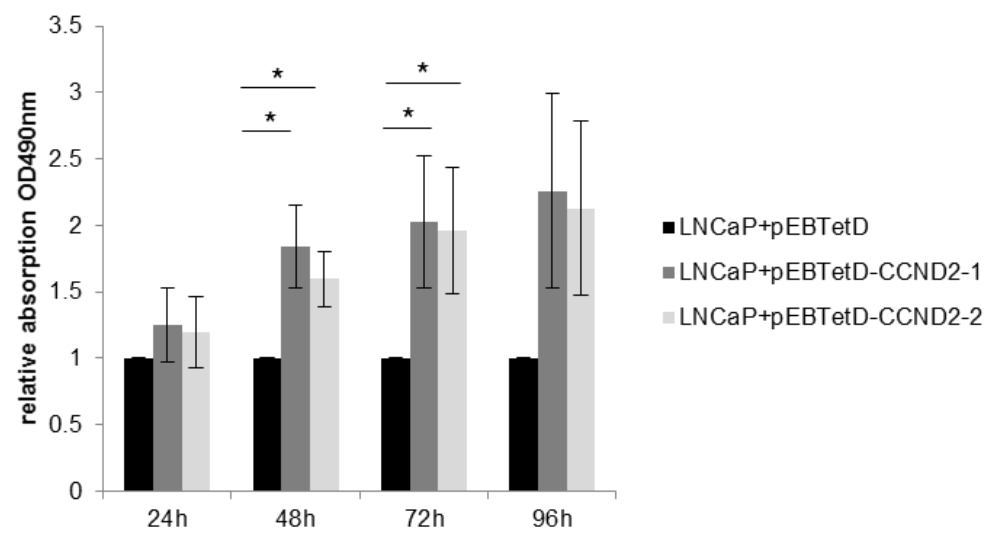

B

LNCaP-pIRES2-EGFP-CCND2

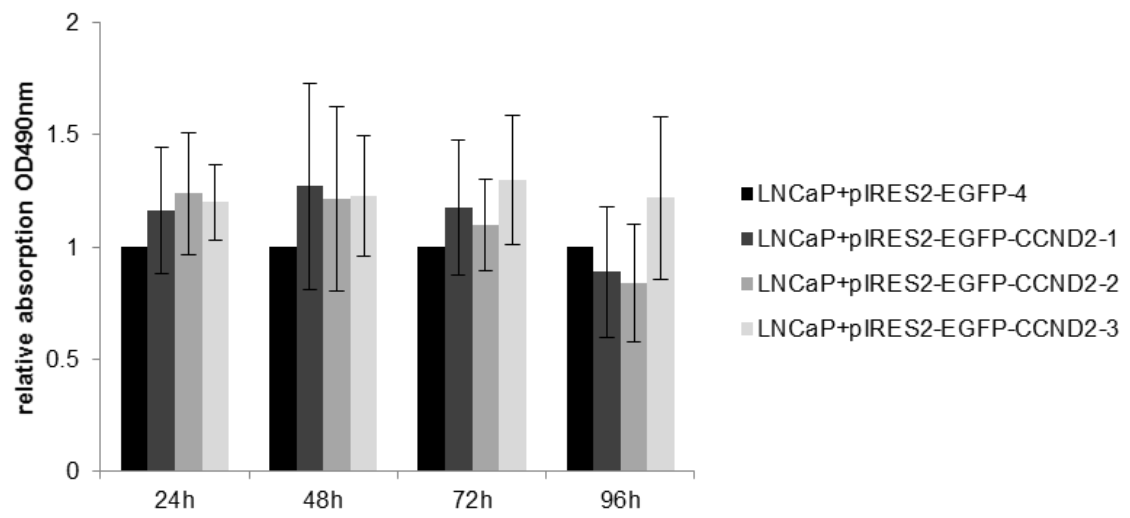

C

PC-3+pIRES2-EGFP-CCND2

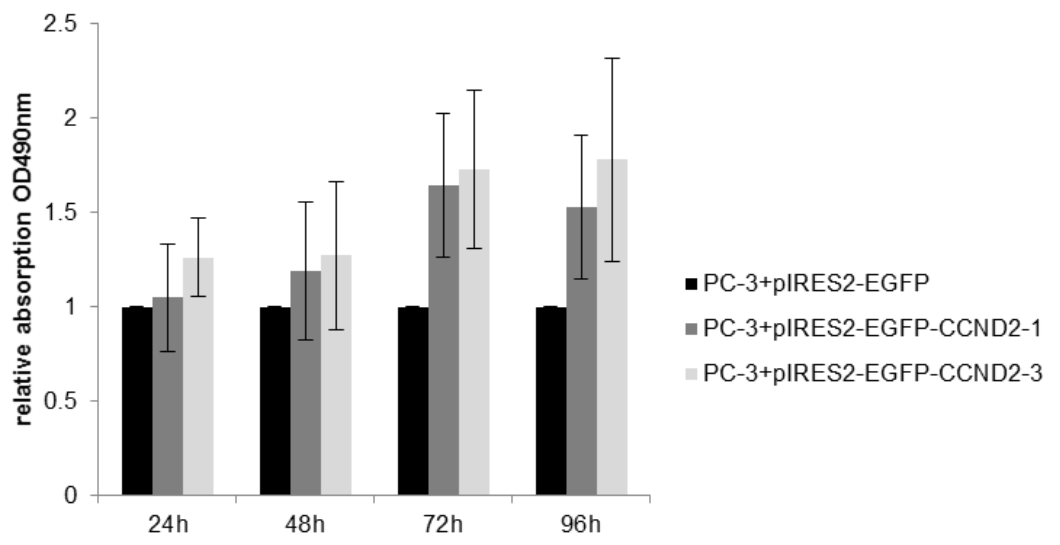

Fig. 3.26: Analysis of the proliferation rate of $\mathrm{PCa}$ cells stably overexpressing cyclin $\mathrm{D} 2$. The proliferation rate of the LNCaP+pEBTetD-CCND2 colonies (A), the LNCaP+pIRES2-EGFP-CCND2 cell clones (B) and the PC3+pIRES2-EGFP-CCND2 cell clones (C) was measured by MTT assay every 24 hours for 5 days. A) The $\mathrm{LNCaP}+\mathrm{pEBTetD}-\mathrm{CCND} 2$ cells were treated with $0.025 \mu \mathrm{g} / \mathrm{ml}$ doxycycline to induce cyclin D2 overexpression. After 48 hours there was a significant increase in the proliferation rate of cells transfected with the cyclin D2 expression vector as compared to the control cells transfected with the empty pEBTetD vector. B) LNCaP-pIRES2-EGFP-CCND2 
cell clones 1, 2 and 3 with a stable cyclin D2 overexpression have a slightly increased proliferation rate, although not statistically significant, as compared to cells of clone 4 without cyclin D2 overexpression. C) The two PC-3+pIRES2EGFP-CCND2 cell clones 1 and 3 with a stable cyclin D2 overexpression showed an increased proliferation rate, although not statistically significant, as compared to cells of clone 4 without cyclin D2 overexpression. Data are presented as mean $+/$ - standard deviation of three proliferation assays. ${ }^{*} 0.01<p \leq 0.05,{ }^{* *} 0.001 \leq p<0.01,{ }^{* * *} p \leq$ 0.001

\subsection{Studies on the migration rate of PCa cells with stable or inducible cyclin D2 overexpression}

The migration ability of the generated LNCaP and PC-3 clones transfected with the pIRES2EGFP-CCND2 expression vector was tested by the transwell migration assay. The cyclin D2 overexpressing clones of both cell lines had an unexpected increased migration rate as compared to control cells (Fig. 3.27).
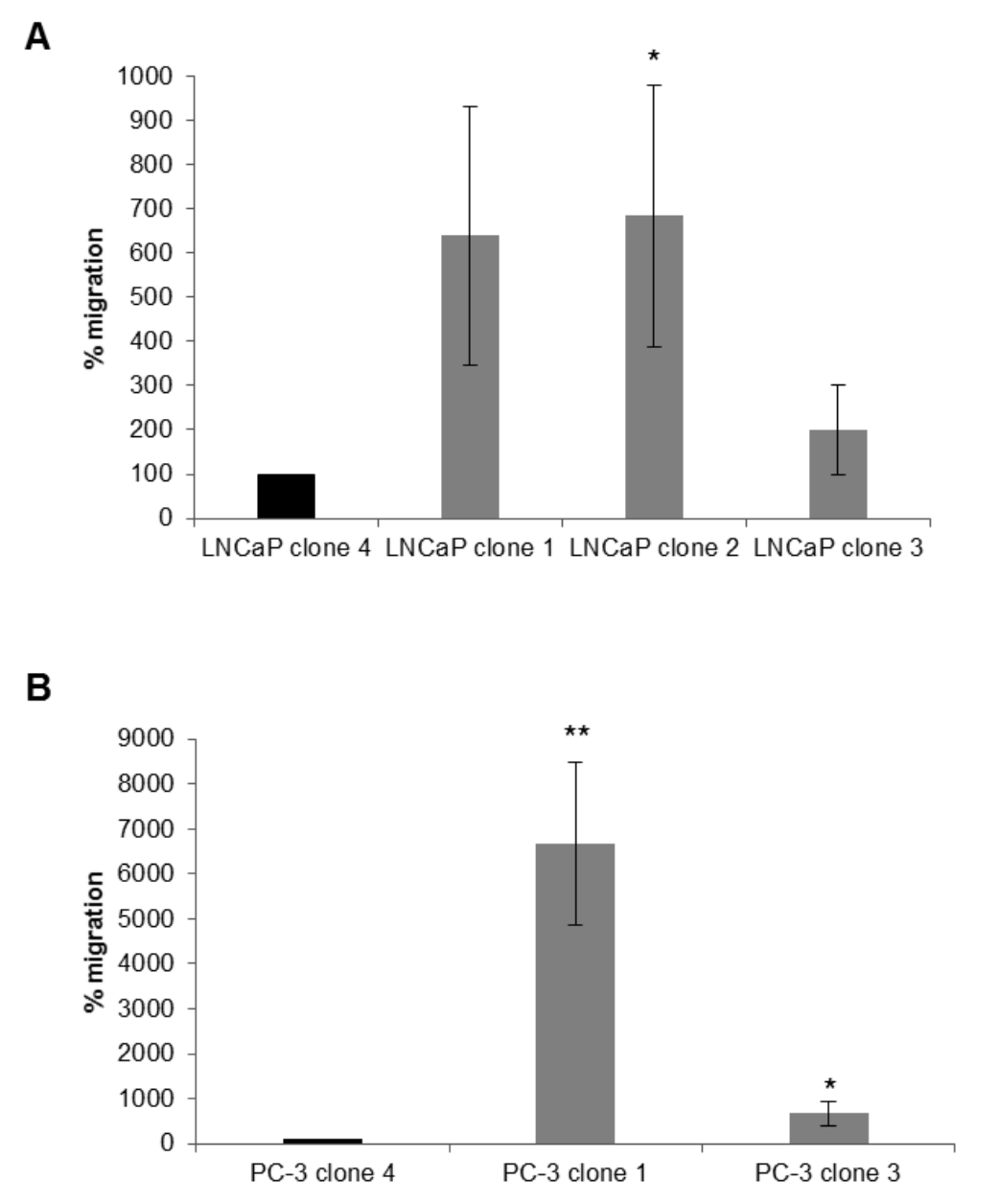

Fig. 3.27: Analysis of the migration rate of PCa cells stably overexpressing cyclin D2. The migration rate of the LNCaP+pIRES2-EGFP-CCND2 cell clones (A) and PC-3+pIRES2-EGFP-CCND2 cell clones (B) was measured by the transwell migration assay 24 hours after the cells were transferred to the assay. The cells were fixed, stained with 
hemalum/eosin and counted under the microscope. A) All three LNCaP cyclin D2 overexpressing cell clones (clone $1,2,3$ ) showed an increase in the proliferation rate as compared to control cells (clone 4) not overexpressing cyclin D2. B) The two PC-3 cell clones with a stable cyclin D2 overexpression showed and increased proliferation rate as compared to cells of clone 4 without cyclin D2 overexpression. Data are presented as mean $+/$ - standard deviation of three migration assays. ${ }^{*} 0.01<p \leq 0.05,{ }^{* *} 0.001 \leq p<0.01,{ }^{* * *} p \leq 0.001$

\subsubsection{Cyclin D2 downregulation studies in NIH/3T3 cells}

In order to verify the hypothesis that cyclin D2 serves as a tumor suppressor gene in PCa previous cyclin D2 overexpression studies were conducted with LNCaP and PC-3 PCa cells from which it was expected that they showed less functional effects of a cancer cell. However, contrary to the expectation and as demonstrated in the previous experiments, their proliferation and migration rate was rather increased. Simultaneously, cyclin D2 downregulation studies were conducted with the mouse fibroblast cell line NIH/3T3 to verify the hypothesis from the other direction. Here, it was expected that cells with reduced cyclin D2 expression exhibit more characteristics of a cancer cell, if cyclin D2 should be a tumor suppressor.

NIH/3T3 cells, which have a high basal cyclin D2 expression, were transfected with different cyclin D2-specific siRNAs or transfected with a generated cyclin D2 shRNA plasmid (pSingletTs-shRNA). Subsequently, the functional effects on migration and invasion behavior were analyzed. If cyclin D2 is indeed a tumor suppressor then these fibroblast cells in which the cyclin D2 expression is downregulated by either siRNA or shRNA should exhibit more features of a cancer cell, e.g. an increased proliferation and migration rate.

\subsubsection{Influence of reduced cyclin D2 expression on migration behavior of NIH/3T3 cells}

The murine fibroblast cells NIH/3T3 were treated with three different siRNAs against cyclin D2 (siRNA A, B, C). Protein of the transfected cells was isolated to test the efficiency of the siRNAs by western blot analysis (Fig. 3.28A). Cyclin D2 siRNA-treated cells had a reduced cyclin D2 expression as compared to luciferase transfected cells, demonstrating the effectiveness of the used siRNAs. Two days after transfection, the migration behavior of the cells was studied by the transwell migration assay. The migration rate of the siRNA-treated NIH/3T3 cells was increased for all three siRNAs tested in comparison to luciferase control transfected cells (Fig. 3.28B). For siRNA B and siRNA C transfected NIH/3T3 cells this effect was statistically very significant. 


\section{A}

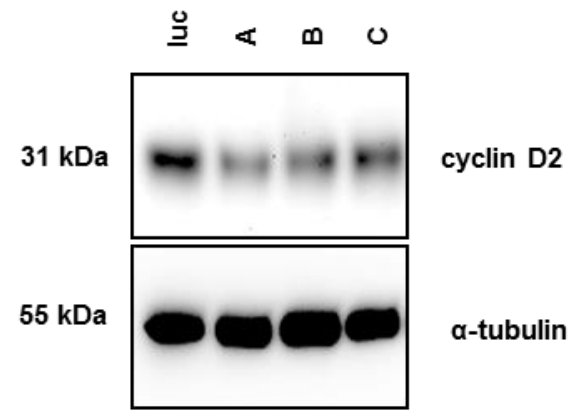

B

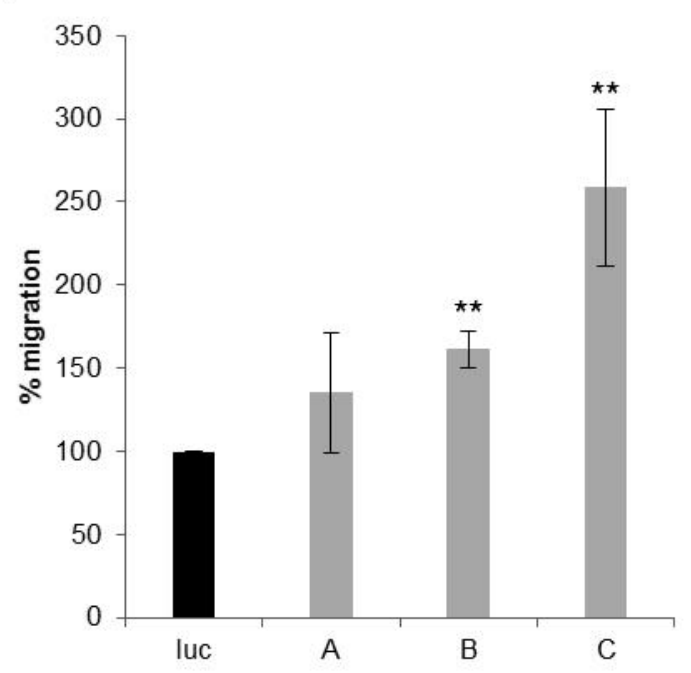

Fig. 3.28: Analysis of the migration rate of $\mathrm{NIH} / 3 \mathrm{~T} 3$ cells after cyclin $\mathrm{D} 2$ downregulation. $\mathrm{NIH} / 3 \mathrm{~T} 3$ cells were treated with three different cyclin D2-specific siRNAs (siRNA A, B, C). 48 hours thereafter the cells were transferred to the transwell migration assay and incubated for 24 hours. The cells were fixed, stained with hemalum/eosin and counted under the microscope. A) Protein of the transfected cells was isolated to test the efficiency of the siRNAs by western blot analysis. Cyclin D2 siRNA-transfected cells had a reduced cyclin D2 expression as compared to luciferase transfected cells, demonstrating the effectiveness of the used siRNAs. B) Treatment of NIH/3T3 cells with either one of the three cyclin D2 siRNAs induced an increase in the migration rate compared to luciferase control transfected cells. Data are presented as mean $+/$ - standard deviation of three independent migration assays. ${ }^{*} 0.01<$ $p \leq 0.05,{ }^{* *} 0.001 \leq p<0.01,{ }^{* * *} p \leq 0.001$

In order to determine whether the increase in the proliferation rate after cyclin D2 siRNA treatment in NIH/3T3 cells is specific for cyclin D2 downregulation, the effect of cyclin D1 downregulation on the migration rate was also studied. The efficiency of the cyclin D1-specific siRNA was tested by western blot analysis (Fig.3.29A) and quantitative real-time PCR (Fig.3.29B). Both, total protein and total RNA lysates were isolated from NIH/3T3 cells 48 hours after cyclin D1 siRNA transfection. The cyclin D1 siRNA proved to be effective as seen by a reduced $C c n d 1$ expression compared to luciferase control transfected cells in the western blot analysis as well as in the quantitative real-time PCR (Fig. 3.29). 
A

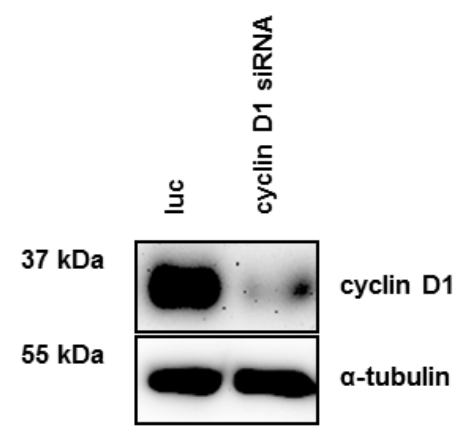

B

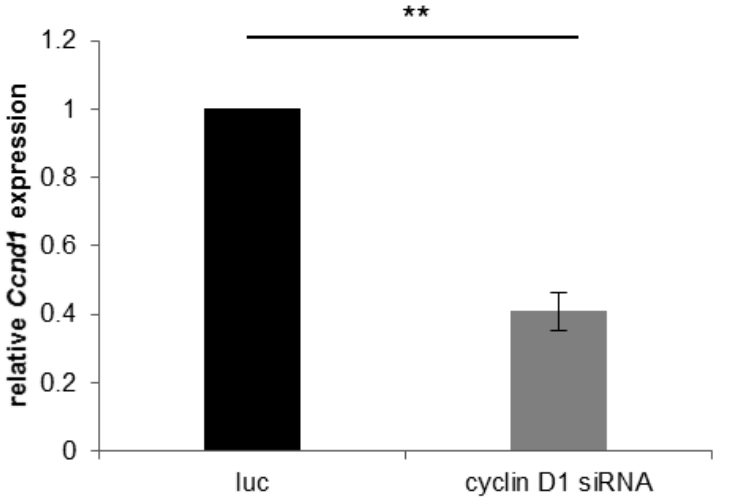

Fig. 3.29: Confirmation of the effectiveness of the cyclin D1-specific siRNA. A) Whole protein lysate of NIH/3T3 cells transfected with a cyclin D1-specific siRNA for 48 hours was immunoblotted using a cyclin D1-specific antibody. Cyclin D1 siRNA treatment reduced the expression of cyclin D1 in comparison to luciferase control transfected cells. Immunoblotting of $\alpha$-tubulin ensured equal protein loading. B) RNA of NIH/3T3 cells transfected with a cyclin D1specific siRNA was isolated 48 hours after transfection and reverse transcribed into CDNA which was subjected to quantitative real-time PCR analysis to demonstrate the effectiveness of the cyclin D1-specific siRNA. Cyclin D1 siRNA-treated cells had a significantly reduced Ccnd1 expression $(p=0.0088)$ as compared to luciferase-transfected cells. Data are presented as mean $+/$ - standard deviation of three quantitative real-time PCR analyses performed in triplicate. ${ }^{*} 0.01<p \leq 0.05,{ }^{* *} 0.001 \leq p<0.01,{ }^{* * *} p \leq 0.001$

After demonstrating that the cyclin D1 siRNA is effectively downregulating Ccnd1 expression the influence on the migration rate of $\mathrm{NIH} / 3 \mathrm{~T} 3$ cells was investigated. No significant difference between the migration rate of cyclin D1 siRNA-transfected and luciferase control-transfected cells was observed (Fig. 3.30).

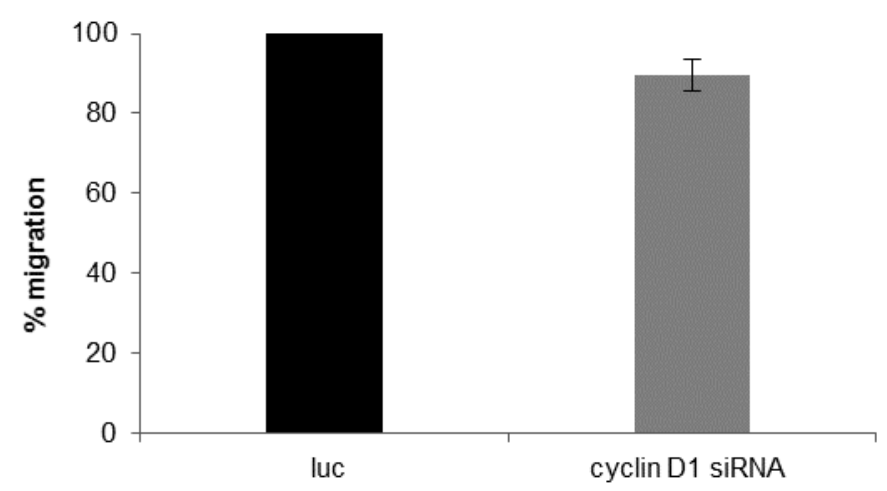

Fig. 3.30: Analysis of the migration rate of $\mathrm{NIH} / 3 T 3$ cells after cyclin $\mathrm{D} 1$ downregulation. $\mathrm{NIH} / 3 T 3$ cells were transfected with a cyclin D1-specific siRNA. 48 hours thereafter the cells were transferred to the transwell migration assay and incubated for 24 hours. The cells were fixed, stained with hemalum/eosin and counted under the microscope. Treatment of $\mathrm{NIH} / 3 \mathrm{~T} 3$ cells with cyclin D1 siRNA induced no changes in the proliferation rate as compared to control cells transfected with luciferase. Data are presented as mean $+/$ - standard deviation of three migration assays. 


\subsubsection{Influence of siRNA-mediated reduced cyclin D2 expression on transformation potential of $\mathrm{NIH} / 3 \mathrm{~T} 3$ cells}

One feature of cancer cells is their ability to grow anchorage-independent which can be investigated by soft agar assay. If the hypothesis is true that cyclin D2 is a tumor suppressor gene, then cells with a reduced or no cyclin D2 expression should exhibit more characteristics of a cancer cell.

To test whether NIH/3T3 cells transfected with a cyclin D2-specific siRNA indeed have the ability to grow anchorage-independent the cells were plated in soft agar and incubated for 4 weeks. During this period of time no anchorage-independent growth of the NIH/3T3 cells with a reduced cyclin D2 expression could be observed (Fig. 3.31). The colon carcinoma cells SW620 cells are known to grow anchorage-independent and served as a positive control (Fig. 3.31, Bullard et al., 2003; Coffey et al., 1986).
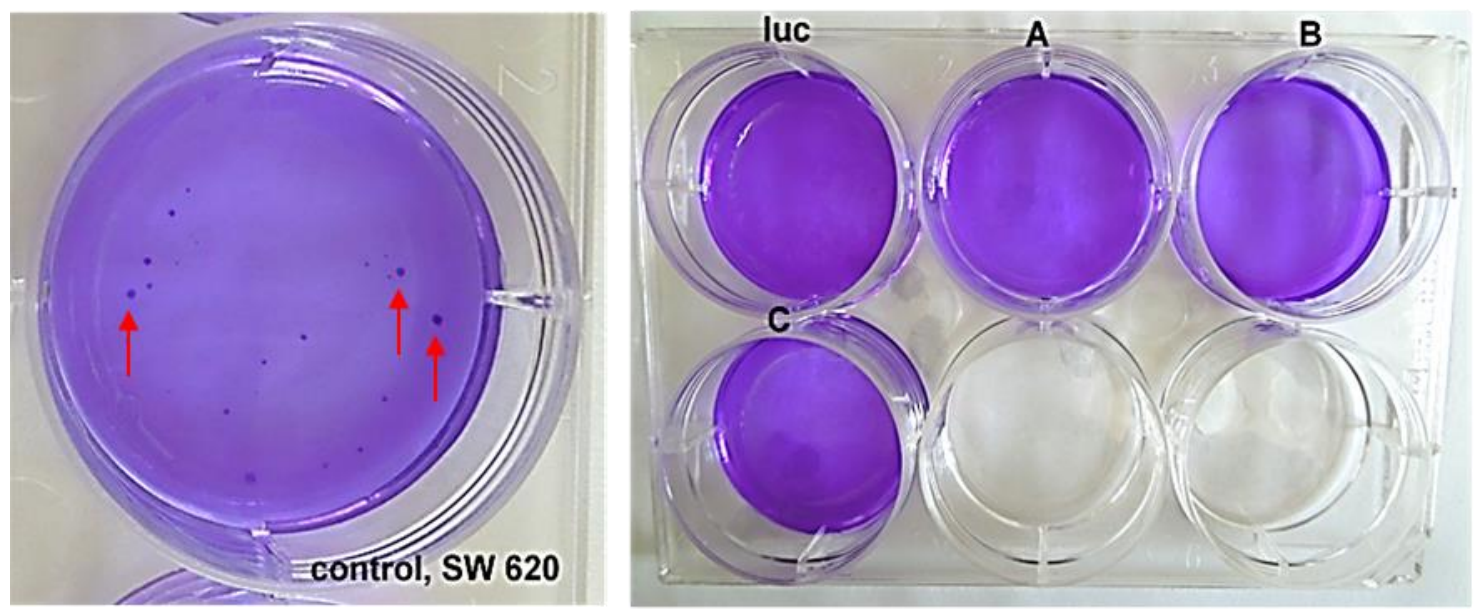

Fig. 3.31: Analysis of the anchorage-independent growth of NIH/3T3 cells after cyclin D2 downregulation. $\mathrm{NIH} / 3 \mathrm{~T} 3$ cells were transfected with three different cyclin D2-specific siRNAs $(A, B, C) .48$ hours thereafter the cells were plated into the top layer of a soft agar assay and incubated for 4 weeks. No anchorage-independent cell growth could be observed in the cyclin D2 siRNA-transfected NIH/3T3 cells. Luciferase control transfected NIH3T3 cells were also not growing in the soft agar assay. The colon carcinoma cells SW620 are known to grow anchorage-independent and served as a positive control. They formed visible colonies already after 2 weeks (red arrows).

To exclude the possibility that anchorage-independent growth of cyclin D2 siRNA-transfected $\mathrm{NIH/3T3}$ cells could not be observed because of a not proper function of the siRNAs the downregulation-efficiency of the three different siRNAs (siRNA A, B, C) on NIH/3T3 cells over a period of three weeks was investigated by western blot analysis. As seen in Fig. 3.32 the initial downregulation of cyclin D2 by either one of the three siRNAs in week one is abolished after three weeks of incubation with the cyclin D2-specifc siRNA. After three weeks siRNA A and C 
can not efficiently suppress the cyclin D2 expression anymore which is seen by a stronger cyclin D2 band in the western blot analysis as compared to the luciferase control transfected cells. Therefore, stable downregulation of cyclin D2 is needed.
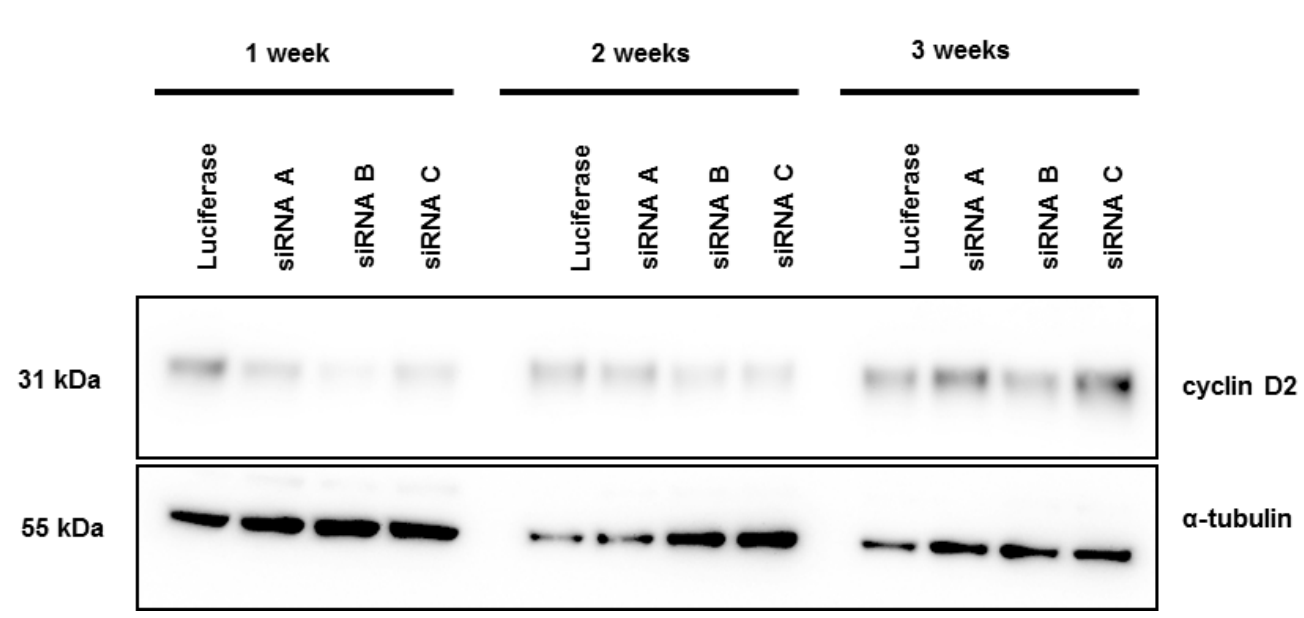

Fig. 3.32: Testing the cyclin D2-specifc siRNA efficiency during a period of three weeks in mouse fibroblast cells. To find out whether siRNA treatment is the appropriate method to test the transformation potential of the mouse fibroblast cells $\mathrm{NIH} / 3 \mathrm{~T} 3$ with a reduced cyclin D2 expression the efficiency of three different cyclin D2-specific siRNAs ( siRNA A, B, C) was tested. Therefore whole protein lysate of NIH/3T3 cells transfected with the cyclin D2-specific siRNA for one, two or three weeks was immunoblotted using a cyclin D2-specific antibody. In the first week, cells treated with either one of the siRNAs had a reduced cyclin D2 expression compared to luciferase control transfected cells. By week three, cells treated with siRNA A or C had an increased cyclin D2 expression compared to the luciferase control transfected cells. Immunoblotting of $\alpha$-tubulin ensured equal protein loading.

\subsubsection{Cyclin D2 shRNA studies in NIH/3T3 cells}

\subsection{Generation of NIH/3T3 cell clones with a stable cyclin D2 downregulation}

Since the downregulation of cyclin D2 in NIH/3T3 cells by the use of siRNAs is not effective and sufficient over longer periods of time (Fig. 3.32) NIH/3T3 cell clones with stable and inducible cyclin D2 downregulation were generated. Therefore, a cyclin D2-specific shRNA construct was cloned into the doxycycline-inducible pSingle-tTs-shRNA vector. Test digestion of isolated plasmid DNA with the restriction enzyme Mlul which has a restriction site exclusively in the shRNA sequence ensured proper integration of the cyclin D2 shRNA in the pSingle-tTS-shRNA plasmid (Fig. 3.33) which was also verified by sequence analysis (data not shown). 


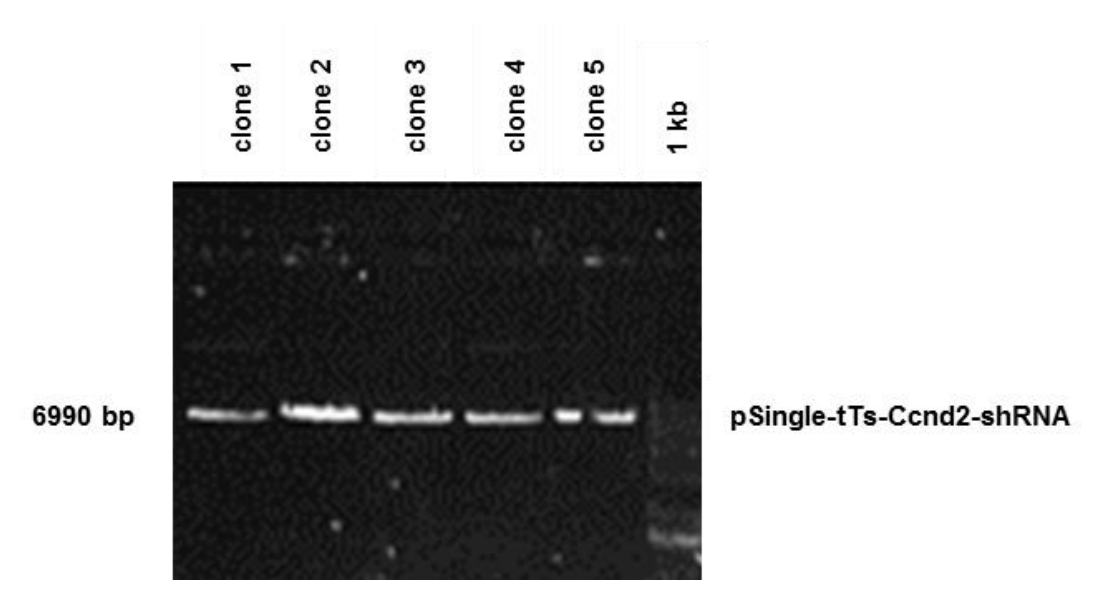

Fig. 3.33: Test digestion of pSingle-tTs-Ccnd2-shRNA clones. Competent DH5 $\alpha$ cells were transformed with the pSingle-tTs-Ccnd2-shRNA vector. Plasmid DNA of single clones was isolated and digested with the restriction enzyme Mlul. The single restriction site of Mlul is located in the shRNA sequence. All of the five clones tested (1-5) could be successfully digested with the restriction enzyme Mlul, indicating the effective integration of the shRNA construct into the pSingle-tTs-shRNA vector. 1kb: 1Kb Plus DNA-ladder

$\mathrm{NIH} / 3 \mathrm{~T} 3$ cells were transfected with the pSingle-tTs-Ccnd2-shRNA vector using Metafectene ${ }^{\circledR}$ Pro transfection reagent. To generate single-cells clones, two days after transfection cells were serially diluted and plated into petri dishes. From the next day on transfected cells were selected by treatment with $400 \mu \mathrm{g} / \mathrm{ml} \mathrm{G} 418$. Colonies of $\mathrm{NIH} / 3 \mathrm{~T} 3$ fibroblast cells expressing the resistance marker could be generated by app. 21 days of growth in medium containing G418 selection agent. Single-cell clones were picked and further cultured in the presence of G418. Total RNA and whole protein lysate were isolated from cell clones to test by quantitative real-time PCR and western blot analysis whether stable transfection was successful and if the cells indeed exhibited a doxycycline inducible downregulation of cyclin D2 by RNA interference. NIH/3T3 cells not treated with doxycycline served as controls.

Subsequently, several different NIH/3T3 clones were tested but only one clone (clone No. 11) showed an efficient downregulation of cyclin D2 after treatment with doxycycline. Quantitative real-time PCR (Fig. 3.34A) demonstrated a downregulation of cyclin D2 expression of about 60 $\%$ in the NIH/3T3 cell clone No. 11 when treated with $0.1 \mu \mathrm{g} / \mathrm{ml}$ doxycycline, as compared to control cells without doxycycline treatment. The downregulation of cyclin D2 after doxycycline treatment with different concentrations was confirmed by western blot analysis (Fig. 3.34B). 
A

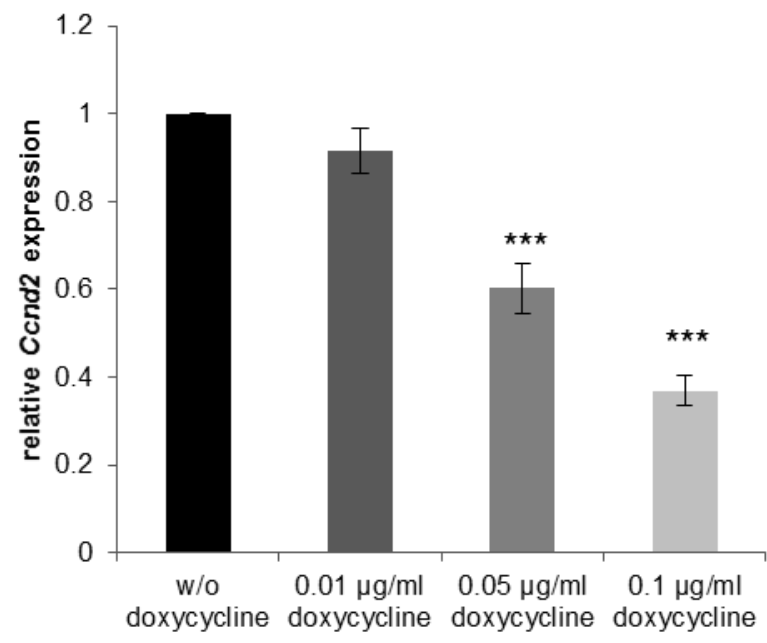

B

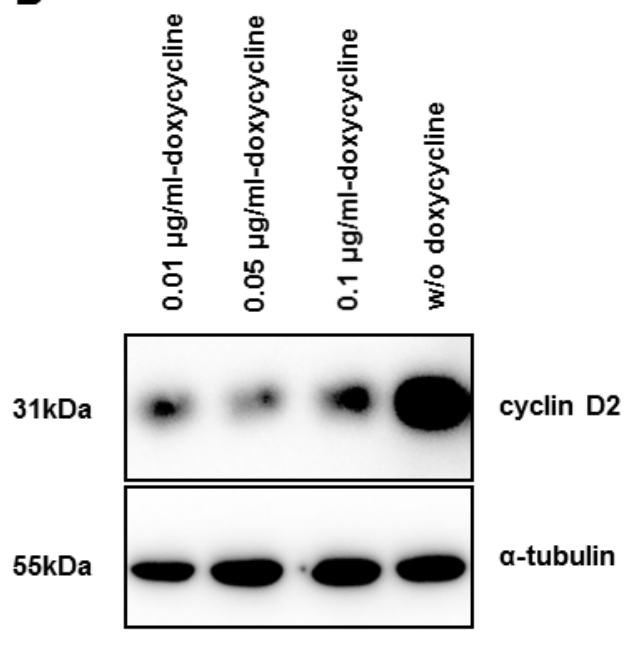

Fig. 3.34: Confirmation of the doxycycline-inducible downregulation of cyclin D2 in mouse fibroblast cells after transfection with pSingle-tTs-Ccnd2-shRNA. Cells of NIH/3T3 clone No. 11 stably transfected with the pSingle-tTs-Ccnd2-shRNA plasmid were treated with three different concentrations of doxycycline $(0.01,0.05$ and 0.1 $\mu \mathrm{g} / \mathrm{ml}$ ) to induce transcription of the cyclin D2 shRNA. 72 hours thereafter total RNA and whole protein lysate were isolated from the cells. A) Of the isolated RNA cDNA was generated by reverse transcription on which the expression of cyclin D2 was tested by quantitative real-time PCR using cyclin D2-specific primers. A reduced cyclin D2 expression of NIH/3T3-pSingle-tTs-Ccnd2-shRNA clone No. 11 after treatment with either one of three different tested doxycycline concentrations compared to the control cells without doxycycline treatment could be observed. Thereby the strongest downregulation of cyclin D2 was seen by the highest doxycycline concentration tested $(0.1 \mu \mathrm{g} / \mathrm{ml})$. Treatment of $\mathrm{NIH} / 3 \mathrm{~T} 3$ clone No. 11 cells with $0.1 \mu \mathrm{g} / \mathrm{ml}$ doxycycline reduced the cyclin D2 expression by about $60 \%$ as compared to cells that were not treated with doxycycline. B) Whole protein lysates were immunoblotted using a cyclin D2-specific antibody. Western blot analysis revealed a reduced cyclin D2 expression of NIH/3T3-pSingle-tTsCcnd2-shRNA clone No. 11 after treatment with either one of three different tested doxycycline concentrations as compared to the control cells without doxycycline treatment, confirming the quantitative real-time PCR data. Immunoblotting of $\alpha$-tubulin ensured equal protein loading. Data are presented as mean $+/$ - standard deviation of three quantitative real-time PCR analyses performed in triplicate. ${ }^{*} 0.01<p \leq 0.05,{ }^{* *} 0.001 \leq p<0.01,{ }^{* * *} p \leq 0.001$

\subsection{Studies on the proliferation rate of $\mathrm{NIH} / 3 \mathrm{~T} 3$ cells with shRNA-mediated cyclin D2 downregulation}

In order to test the hypothesis that cyclin D2 displays the features of a tumor suppressor gene then NIH/3T3 cells with reduced cyclin D2 expression (in this case by shRNA) should exhibit more characteristics of cancer cells, e.g. an increased proliferation rate as compared to $\mathrm{NIH} / 3 \mathrm{~T} 3$ cells with a normal cyclin D2 expression. Therefore, cells of the generated NIH/3T3-pSingle-tTsCcnd2-shRNA clone No. 11 were plated into 96-well plates. The next day cells were treated with three different concentrations of doxycycline $(0.05 \mu \mathrm{g} / \mathrm{ml} ; 0.1 \mu \mathrm{g} / \mathrm{ml} ; 1.0 \mu \mathrm{g} / \mathrm{ml})$ as well as with 
the PMS/MTS solution of the MTT assay. Two hours later the first proliferation was measured and then every 24 hours for 4 consecutive days by a MTT assay. The doxycycline treated cells had a slight tendency to a reduced, although not statistically significant, proliferation rate as compared to control cells which were not treated with doxycycline (Fig. 3.35).

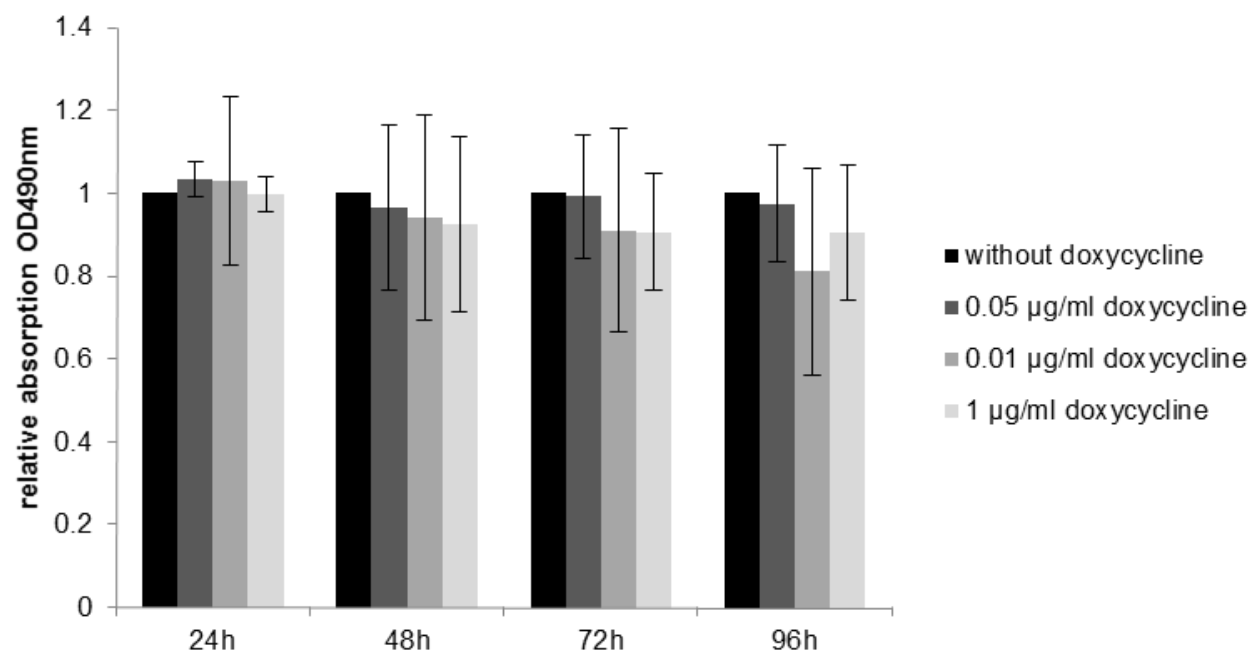

Fig. 3.35: Analysis of the proliferation rate of $\mathrm{NIH} / 3 \mathrm{~T} 3$ cells with an inducible cyclin D2 downregulation. The proliferation rate of NIH/3T3-pSingle-tTs-Ccnd2-shRNA clone No. 11 cells was measured by a MTT assay every 24 hours after plating the cells to the 96-well plate for 5 consecutive days. On the first day of the measurement the cells were treated with three different concentrations of doxycycline $(0.05 \mu \mathrm{g} / \mathrm{ml} ; 0.1 \mu \mathrm{g} / \mathrm{ml} ; 1.0 \mu \mathrm{g} / \mathrm{ml})$ to induce cyclin D2 shRNA expression. NIH/3T3 cells not treated with doxycycline served as a control. Overall, the doxycycline treatment of NIH/3T3-pSingle-tTs-Ccnd2-shRNA clone No. 11 resulted in a reduced, although not statistically significant, proliferation rate as compared to control cells which were not treated with doxycycline. Data are presented as mean + /- standard deviation of three proliferation assays.

\subsection{Influence of shRNA-mediated reduced expression of cyclin D2 on the transformation potential of $\mathrm{NIH} / 3 \mathrm{~T} 3$ cells}

The previous soft agar assay performed with cyclin D2 siRNA-transfected NIH/3T3 cells revealed no transformation potential of these cells, presumably because siRNA transfection is not effective for downregulation of cyclin D2 over a longer time period. Therefore, the soft agar assay was repeated with cells of NIH/3T3-pSingle-tTs-Ccnd2-shRNA clone No. 11 treated with doxycycline to induce stable cyclin D2 downregulation. Control cells were not treated with doxycycline. But again, even with stable cyclin D2 downregulation no transformation potential by anchorage-independent growth of the NIH/3T3-pSingle-tTs-Ccnd2-shRNA clone No. 11 cells after a four week incubation period could be observed (data not shown). Therefore, NIH/3T3pSingle-tTs-Ccnd2-shRNA clone No. 11 cells were additionally transiently transfected with pEGFP-KRAS and pEGFP-KRAS-G12V, which was shown to induce anchorage-independent 
growth in fibroblasts (Barr and Johnson 2001, Song et al. 2002, Hoque et al. 2006). As a control cells of the NIH/3T3-pSingle-tTs-Ccnd2-shRNA clone No. 11 were transiently transfected with the empty pEGFP plasmid. The soft agar assay was done after 48 hours of doxycycline treatment with a concentration of $0.1 \mu \mathrm{g} / \mathrm{ml}$. The NIH/3T3- pSingle-tTs-Ccnd2-shRNA clone 11 alone acquired no transformation potential, since no colonies were growing in the soft agar (Data not shown). The soft agar assay with the KRAS oncogene transfected cells could not be evaluated since the transfection efficiency was not sufficient (data not shown).

\subsubsection{Studies on the functional consequences of cyclin D2 downregulation in VPA- treated PCa cells}

Witt et al. (2013) could show that VPA treatment of PCa cells specifically led to a re-expression of cyclin D2. Because the cyclin D2 promoter region is hypermethylated cyclin D2 expression is downregulated in PCa. Treatment of PCa cells with the histone deacetylase inhibitor VPA induced increased acetylation of the cyclin D2 promoter and consequently re-expression of cyclin D2 (Witt et al., 2013). Witt et al. could also show that VPA treatment of PCa cells reduced the ability of the cells to proliferate, a characteristic of cancer cells. To study the possible connection between the increased cyclin D2 expression and the reduced proliferation of PCa cells after VPA treatment an experiment was conducted where human and mouse PCa cells (LNCaP, DU145, PC-3 and 2E) were first transfected with two different cyclin D2-specific siRNAs and at the following day transfected cells were treated with different concentrations of VPA (1mM and $5 \mathrm{mM}$ ). Simultaneously, the proliferation rate was measured by a MTT assay. Cyclin D2 siRNA treatment was supposed to prevent the VPA-induced re-expression of cyclin D2 and should thereby lead to an increase in the proliferation in case cyclin D2 would be responsible for the VPA-induced reduced proliferation of PCa cells. Since the efficiency of the three mouse cyclin D2-specific siRNAs had already been tested by western blot on proteins isolated from mouse NIH/3T3 cells transfected with the siRNAs (see Fig. 3.28A), the human cyclin D2-specific siRNAs (siRNA A, B, C) still had to be tested regarding their efficient downregulation of cyclin D2. Therefore, the human colon epithelial cancer cell line CaCo-2, which is known to express human cyclin D2, were transfected with the human cyclin D2-specific siRNAs and 120 hours later total amount of protein was isolated and immunoblotted with a cyclin D2-specific antibody. Human cyclin D2 siRNA A and B led to a sufficient downregulation of cyclin D2 whereas siRNA $C$ led only to a minor downregulation of cyclin D2 as compared to the luciferase control transfected cells (Fig. 3.36). For the following study of simultaneous cyclin D2 siRNA treatment and VPA treatment only human cyclin D2 specific siRNA A and B were used. 


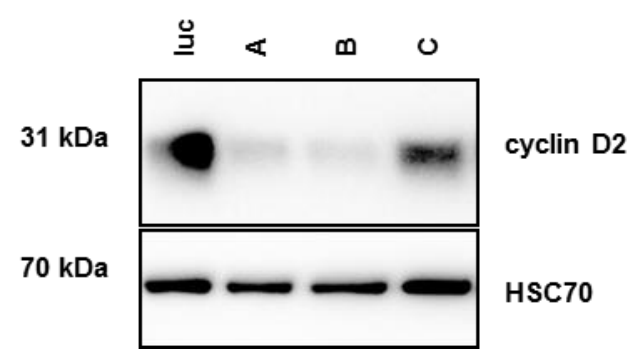

Fig. 3.36: Testing the efficiency of three different human cyclin D2-specific siRNAs in CaCo2 cells. Human colon epithelial cancer cells CaCo-2 were transfected with three different cyclin D2-specific siRNAs (A, B, C) and 120 hours later protein was isolated from the cells. Whole protein lysate was immunoblotted using a cyclin D2-specific antibody. Transfection with either siRNA A or siRNA B led to an efficient cyclin D2 downregulation as compared to the luciferase control transfected cells. Transfection with siRNA C had only minor effects on cyclin D2 downregulation. Immunoblotting of $\mathrm{HSC70}$ ensured equal protein loading.

After testing the efficiency of the human cyclin D2-specific siRNAs human and mouse PCa cells (LNCaP, DU145, PC-3 and 2E) were transfected with two different cyclin D2 specific siRNAs (LNCaP, DU145, PC-3 cells: human-specific siRNAs A and B; 2E cells: mouse-specific siRNAs $B$ and $C$ ) and controls were transfected with luciferase. The following day, the transfected cells were treated with different concentrations of VPA $(1 \mathrm{mM}$ and $5 \mathrm{mM})$ before the proliferation rate was measured by a MTT assay for 96 consecutive hours (Fig 3.37).

Control cells that were transfected only with the siRNA but were not treated with VPA exhibited a reduced proliferation rate as compared to luciferase transfected cells during the four day study. In PC-3 and LNCaP cells this effect was time-dependent. An increased proliferation rate as compared to the control was observed in $2 \mathrm{E}$ cells after 48 and 96 hours and in LNCaP cells during the first two days, although this was a minor effect (Fig.37, left panel). Upon VPA treatment of all four cell lines their proliferation rate decreased further as compared to the controls in a concentration-dependent manner (only shown four one time point (48 hours), Fig.37, right panel). The only exception here was observed in PC-3 cells transfected with siRNA $B$ and treated with $1 \mathrm{mM}$ VPA, where the proliferation rate slightly increased.

Here it was expected, that cells which have a reduced cyclin D2 expression due to siRNAtransfection would have an increased or comparable proliferation rate to controls upon VPA treatment. The known increased cyclin D2 expression after VPA treatment should be prevented by the siRNA which should lead to an increased proliferation rate in case cyclin D2 is directly responsible for the decreased proliferation rate observed in 2E cells (Witt et al., 2013). But contrary to the expectation, VPA treatment of the siRNA-transfected cells further decreased the proliferation rate. This could be due to the fact that downregulation of cyclin D2 expression via siRNA is not sufficient to prevent VPA-induced re-expression of cyclin D2. This assumption is 
supported by the observation from a quantitative real-time PCR analysis which showed that cyclin D2 siRNA-transfected 2E cells still exhibited an increased cyclin D2 expression upon VPA treatment as compared to controls by using quantitative real-time PCR analysis (data not shown).

controls w/o VPA
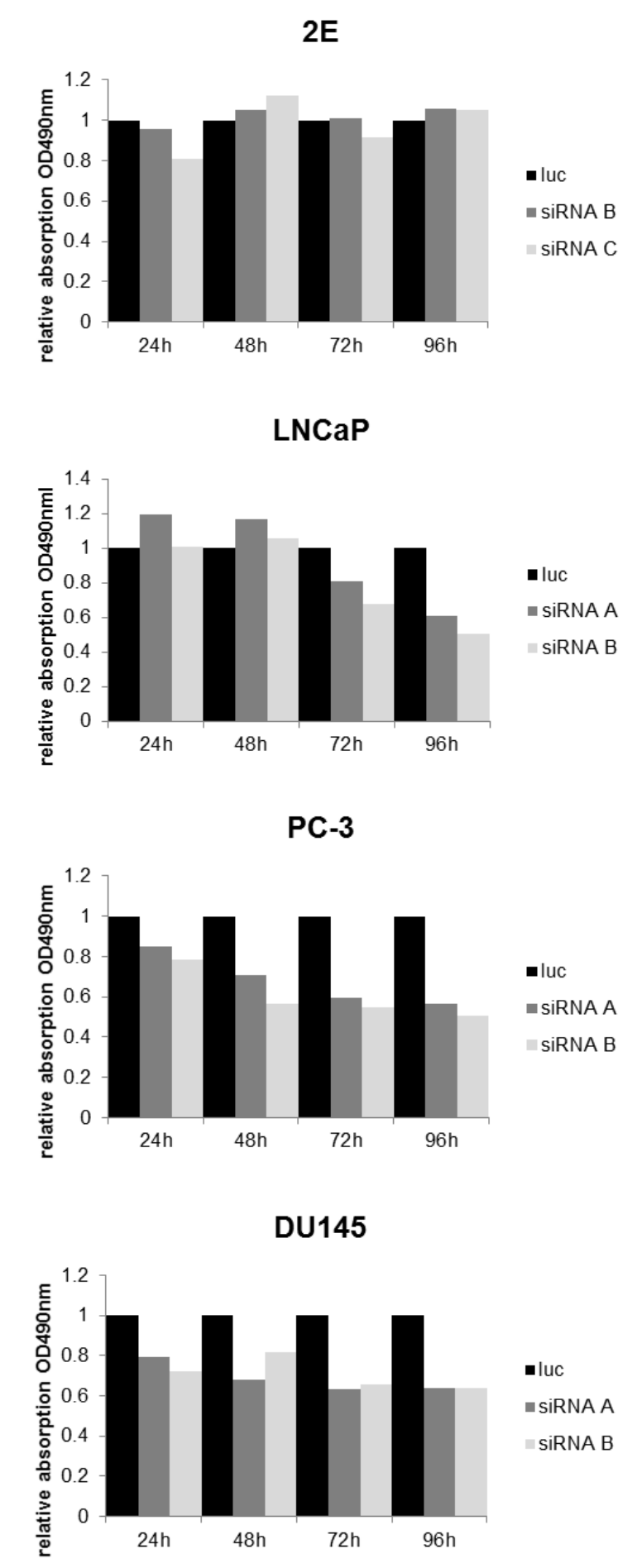

one time point (48h) with VPA
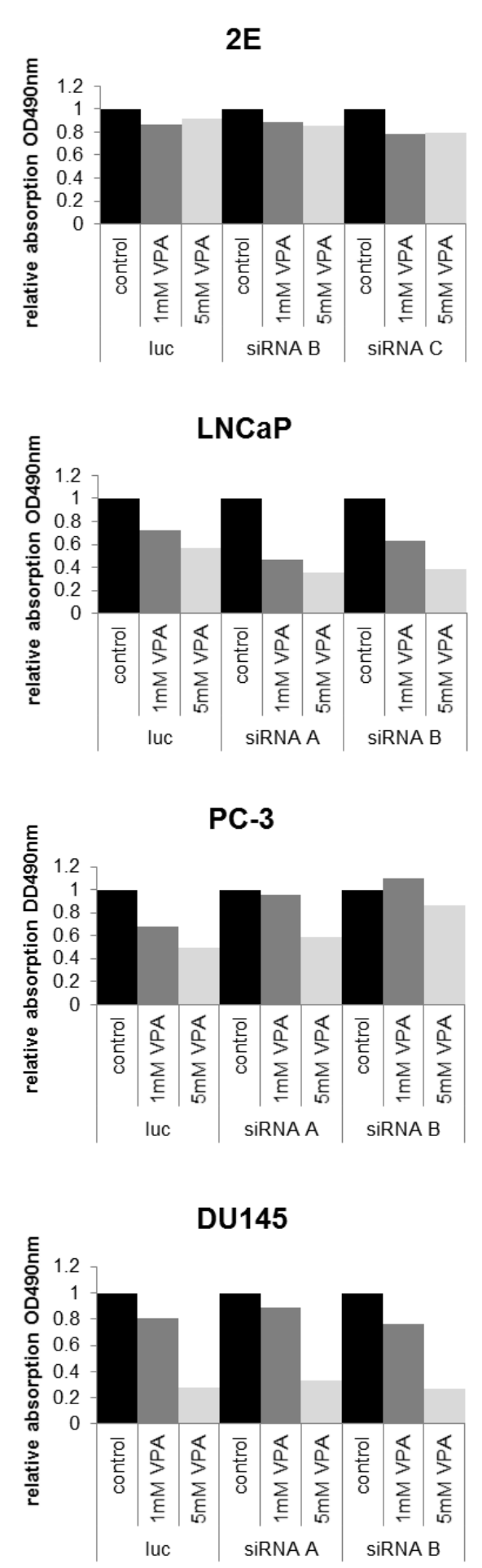
Fig. 3.37: Analysis of the proliferation rate in VPA-treated PCa cells with a reduced cyclin D2 expression. The mouse PCa cells 2E and the human PCa cells PC-3, LNCaP and DU145 were transfected with two different cyclin D2-specific siRNAs (human: siRNA A and B; mouse: siRNA B and C). Cells transfected with luciferase served as control. The day following transfection the cells were treated with either $1 \mathrm{mM}$ or $5 \mathrm{mM}$ VPA. Controls were not treated with VPA. Simultaneously, PMS/MTS solution was added to the cells and two hours later the measurement of the proliferation rate started by a MTT assay, which was conducted for 96 consecutive hours. Here, the proliferation rate for transfected control cells (no VPA treatment) over the time course is depicted on the left panel. The human PCa cell lines transfected with either one of the two siRNAs exhibit a reduced proliferation rate as compared to luciferasetransfected cells, which was time-dependent in PC-3 and LNCaP cells. In 2E cells, after 48 and 96 hours and in LNCaP cells during the first 48 hours the proliferation rate is slightly increased as compared to controls. On the right panel, the proliferation rate for the four cell lines upon VPA treatment is represented for one time point, namely after 48 hours. In all cell lines analyzed, the proliferation rate decreased further upon VPA treatment in a concentrationdependent manner as compared to untreated controls. Solely in PC-3 cells transfected with siRNA B and treated with $1 \mathrm{mM}$ VPA a slight increase in the proliferation rate was observed. This experiment was conducted once.

\subsubsection{Generation of a conditional cyclin D2 knockout mouse model}

\subsubsection{Establishment of the $C c n d 2^{f / f l}$ and $P B-C r e 4^{+}$mouse lines}

To investigate the function of cyclin D2 in vivo a conditional cyclin D2 knockout mouse model was generated. The Cre-Lox system relies on the DNA recombinase Cre and its recognition (loxP) sites. For conditional mutagenesis, a target gene is modified by the insertion of two loxP sites that enable the excision of the flanked (floxed) gene segment through Cre-mediated recombination. Conditional mutant mice are obtained by crossing the floxed strain with a Cre transgenic mouse line such that the target gene becomes inactivated in vivo within the expression pattern of Cre (Friedel et al., 2011).

Two male homozygous $C c n d 2^{f / f t}$ mice with a $C 57 \mathrm{bl} / 6 \mathrm{~N}$ background were obtained from Peter Sicinski at the Dana Faber Institute in Boston, USA. The scheme of the cyclin D2 floxed construct is depicted in Fig. 3.38A. Exon 1 and 2 are flanked by loxP sites and will be excised upon Cremediated recombination. A male and a female heterozygous $P B-C r e 4^{+}$mouse were obtained from Xiantuo Wu, Keck School of Medicine, University of Southern California, Los Angeles, USA (Wu et al., 2001). This established mouse line carries the Cre gene under the control of a composite promoter, ARR2PB, containing two tandem repeats of the androgen responsive region and $\mathrm{PB}$, which is a derivative of the rat prostate-specific probasin (PB) promoter. It ensures specific post-natal expression only in prostate epithelial cells. The scheme of the constructed cassette (ARR2PB-Cre-SV40) is depicted in Fig. 3.38B. 

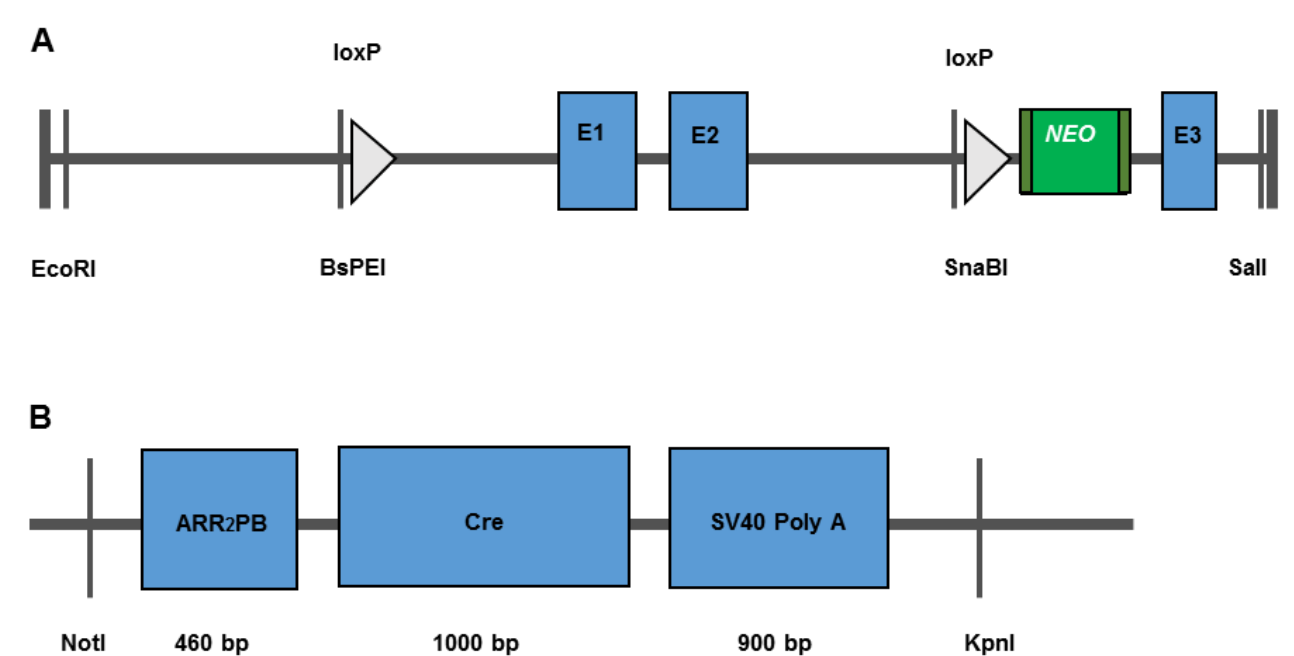

Fig. 3.38: Scheme of the cyclin D2 floxed construct and the Cre construct. A) The cyclin D2 target gene was modified by P.Sicinski (Dana Faber Institute, Boston, USA) by the insertion of two loxP sites flanking Exon 1 and Exon 2. These loxP sites can be recognized by the Cre-recombinase and excised upon Cre-mediated recombination. B) The Cre transgene construct carries the Cre gene under the control of the composite ARR2PB promoter which is a derivative of the rat prostate-specific probasin (PB) promoter, followed by a SV40 polyadenylation sequence. The PB promoter ensures specific post-natal expression only in prostate epithelial cells.

To establish a colony of the purchased $C c n d 2^{f / f t / t}$ mice, the obtained two male mice were mated with female C56bl/6N wild type mice (Fig. 3.39A). In the $\mathrm{F} 1$ generation heterozygous Ccnd2 $2^{f / /}$ mice were obtained which were mated with heterozygous $C c n d 2^{\text {ff/ } /}$ mice from the second line to generate homozygous Ccnd $2^{t / f / t}$ mice (Fig.3.39A).

For the establishment of a colony of the purchased $P B-C r e 4^{+}$mice, the heterozygous male and female mice were mated with female and male $C 56 \mathrm{bl} / 6 \mathrm{~N}$ wild type mice, respectively (Fig.3.39B). The genotype of the offspring was determined by genotyping PCR. Therefore, genotyping-specific primers were used on genomic DNA isolated from a mouse tail biopsy. Cyclin D2 floxed-specific primers amplified a 260 bp product from the wild type allele and a 500 bp product from the floxed allele (Fig. 3.39A). The Cre-specific primers amplified an app. $500 \mathrm{bp}$ product from the Cre allele (Fig.3.39B). 
A
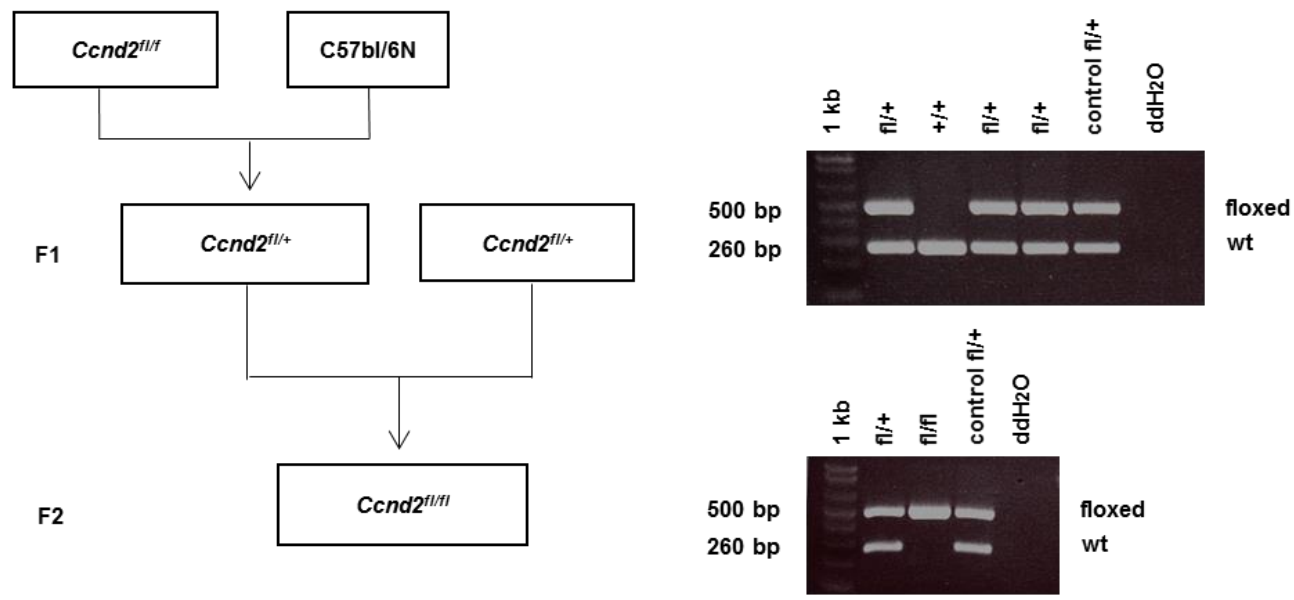

B
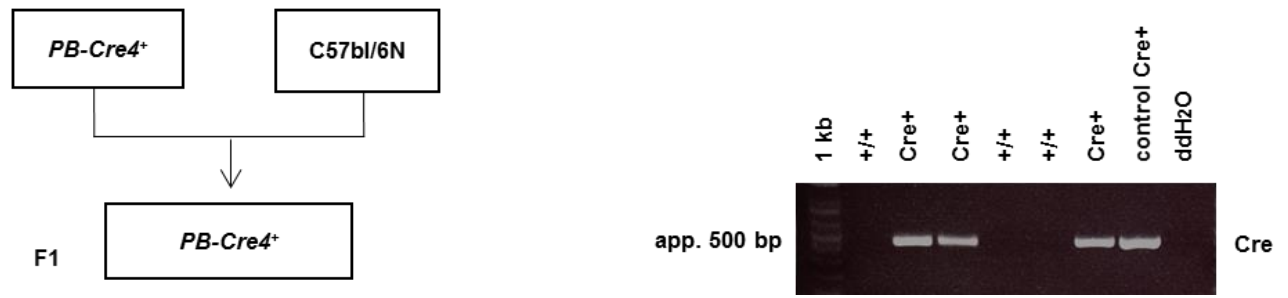

Fig. 3.39: Mating scheme for the establishment of the $C c n d 2^{f / f l}$ and $P B-C r e 4^{+}$mouse lines and genotyping PCR. The obtained Ccnd2f//fl mice (A) as well as the PB-Cre4+ mice (B) were mated to C57bl/6N wild type mice to establish a colony of these lines. The genotype of the mice was determined by genotyping PCR using genotypingspecific primers and genomic DNA derived from a tail biopsy. Cyclin D2-floxed specific primers identified a $260 \mathrm{bp}$ product from the wild type $(\mathrm{wt})$ allele and a $500 \mathrm{bp}$ product from the floxed allele. The Cre-specific primers identified an app. 500 bp product from the Cre allele. A known heterozygous sample was used as positive control and dd $\mathrm{H}_{2} \mathrm{O}$

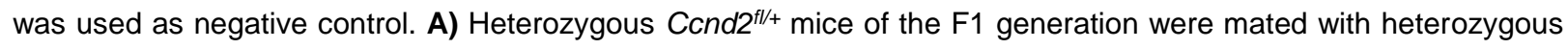
$C c n d 2^{f / /+}$ mice to receive homozygous $C c n d 2^{f / f t}$ mice again in the F2 generation. B) The obtained heterozygous $P B$ Cre $4^{+}$mice were mated with $\mathrm{C} 57 \mathrm{bl} / 6 \mathrm{~N}$ wild type mice to establish a colony of heterozygous $P B-C r e 4^{+}$mice. $1 \mathrm{~kb}: 1$ $\mathrm{Kb}$ Plus DNA-ladder

\subsubsection{Generation of $\mathrm{Ccnd}^{\mathrm{flft} / \mathrm{Cre} \mathrm{C}^{+} \text {mice }}$}

According to Birbach, female PB-Cre4 mice can display aberrant Cre activity due to Cre expression in the oocyte (Birbach, 2013). When transmitted through male mice the Cre expression cassette is exclusively expressed in the prostate epithelium. However, when transmitted through female mice, recombination with loxP-flanked alleles can occur in many different tissues, resulting in different outcomes for maternally or paternally transmitted loxP. flanked alleles. Paternally transmitted loxP-flanked alleles will give rise to mosaic expression 
patterns in the offspring since they undergo recombination inefficiently, whereas maternally transmitted loxP-flanked alleles undergo recombination very efficiently (monoallelic Cre deleted line, Birbach, 2013). To avoid aberrant Cre-activity in the conditional cyclin D2 knockout mice female $C c n d 2^{f / f t}$ had to be mated to male PB-Cre4+ mice (Fig.3.40).

For the main mating homozygous female $C c n d 2^{f / f t}$ mice were bred with heterozygous male $P B$ $\mathrm{Cre}^{+}$mice to generate heterozygous $\mathrm{Ccnd}^{\mathrm{fl} /+} / \mathrm{Cre}^{+}$mice in the $\mathrm{F} 1$ generation. Male Ccnd ${ }^{f / /} / \mathrm{Cre}^{+}$mice were then crossed again with homozygous female $\mathrm{Ccnd} 2^{f / / f l}$ mice to generate homozygous Ccnd2 $2^{f / f t} / \mathrm{Cre}^{+}$conditional knockout mice in the F2 generation (Fig. 3.40A). The genotype of the offspring was determined by genotyping PCR using genotyping-specific primers. Genomic DNA was isolated from a mouse tail biopsy and then subjected to two different genotyping PCRs (Fig. 3.40B). Using cyclin D2 floxed-specific primers a 260 bp product from the wild type allele, a 500 bp product from the floxed allele and a 423 bp product from the deleted allele were amplified, respectively. The Cre-specific primers amplified an app. 500 bp product from the Cre allele. Animal numbers 123 and 124 show the desired $C c n d 2^{f / f t} / C e^{+}$genotype (Fig. $3.40 \mathrm{~B})$.

A

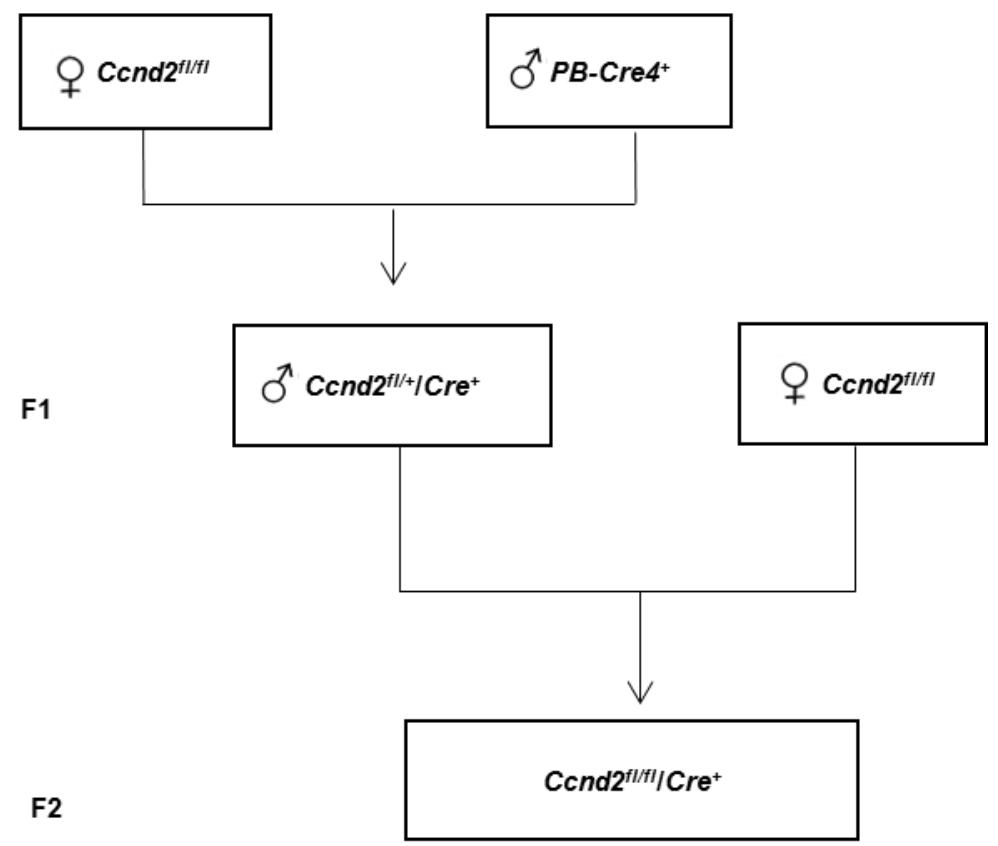




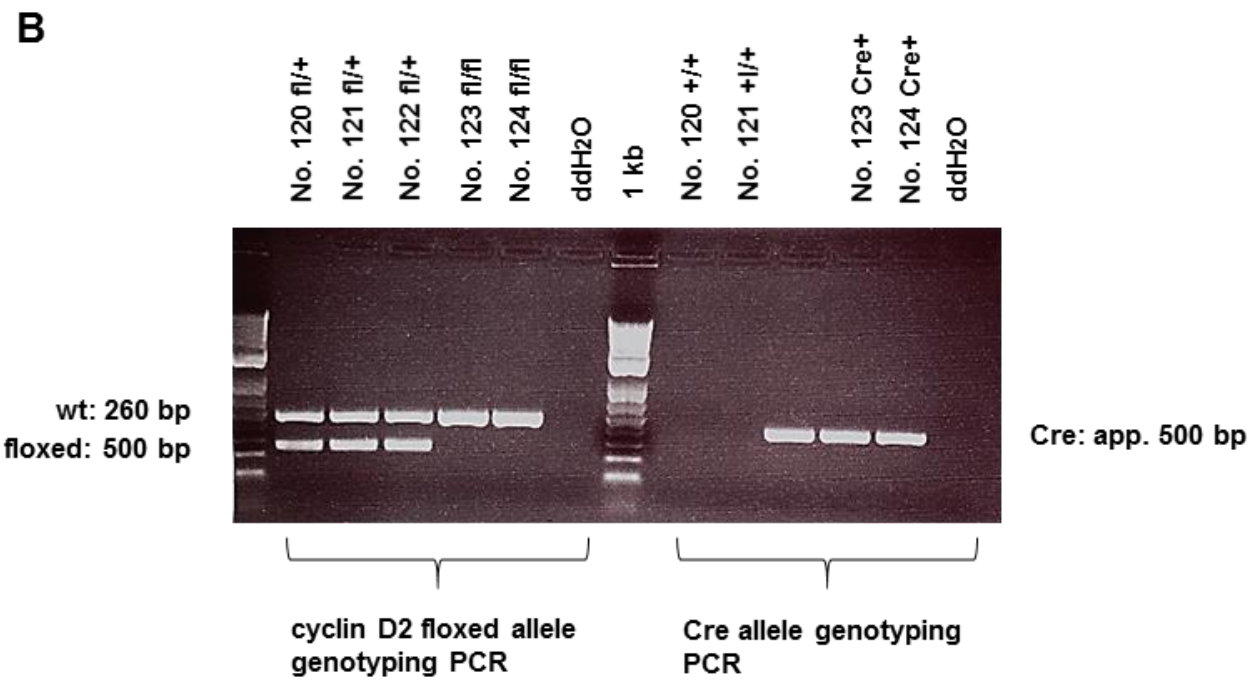

Fig. 3.40: Mating scheme for the generation of homozygous conditional cyclin D2 knockout mice and genotyping PCR. A) Female homozygous $C c n d 2^{f / f t}$ mice were mated with male heterozygous $\mathrm{PB}$-Cre $4^{+}$mice. Male heterozygous $\mathrm{Ccnd} 2^{f /+} / \mathrm{Cre} e^{+}$mice obtained in the $\mathrm{F} 1$ generation were further mated to homozygous $\mathrm{Ccnd} 2^{t / f / t}$. The F2

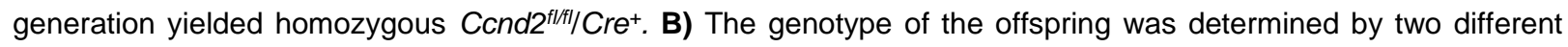
genotyping PCRs using genotyping-specific primers. Genomic DNA was derived from a tail biopsy. In one PCR cyclin D2 floxed-specific primers were used and amplified a 260 bp product from the wild type (wt) allele, a 500 bp product from the floxed allele and a $423 \mathrm{bp}$ produced from the deleted allele. In the second PCR Cre-specific primers were used and amplified an app. 500 bp product from the Cre allele. $\mathrm{ddH}_{2} \mathrm{O}$ was used as negative control. Animal numbers 123 and 124 have the desired Ccnd $2^{f / f / l} / \mathrm{Cre}^{+}$genotype. $1 \mathrm{~kb}: 1 \mathrm{~Kb}$ Plus DNA-ladder

\subsubsection{Verification of the prostate-specific cyclin D2 knockout}

Genomic DNA of different mouse tissues, including testis, kidney, liver, intestine, spleen and prostate, from putative heterozygous and homozygous conditional cyclin D2 knockout mice was analyzed to verify the prostate-specific cyclin D2 knockout.

For the PCR analysis of conditional knockout mice the genotypic-specific primers were used: D2 FLOX 3', D2 FLOX5' and D2 Delta. These primers amplified a 500 bp product from the D2 floxed allele, a 423 bp product from the knockout allele (both encircled in green, Fig.3.41) and a 260 bp product from the wild type sequence in the tissue of heterozygous Ccnd2 $2^{\mathrm{fl}+} / \mathrm{Cre}^{+}$mice (Fig.

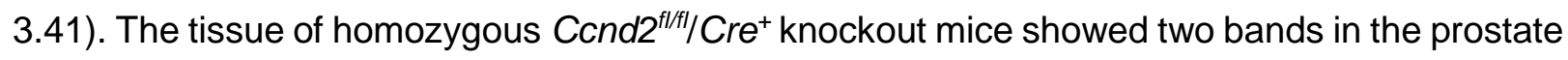
where only one band for the deleted cyclin D2 allele was expected (423 bp, Fig. 3.41). This observation is probably due to the fact that DNA from a mixture of cells (e.g. epithelial cells and stromal cells) from the mouse prostate was analyzed and not exclusively pure epithelial cells from the prostate gland. 


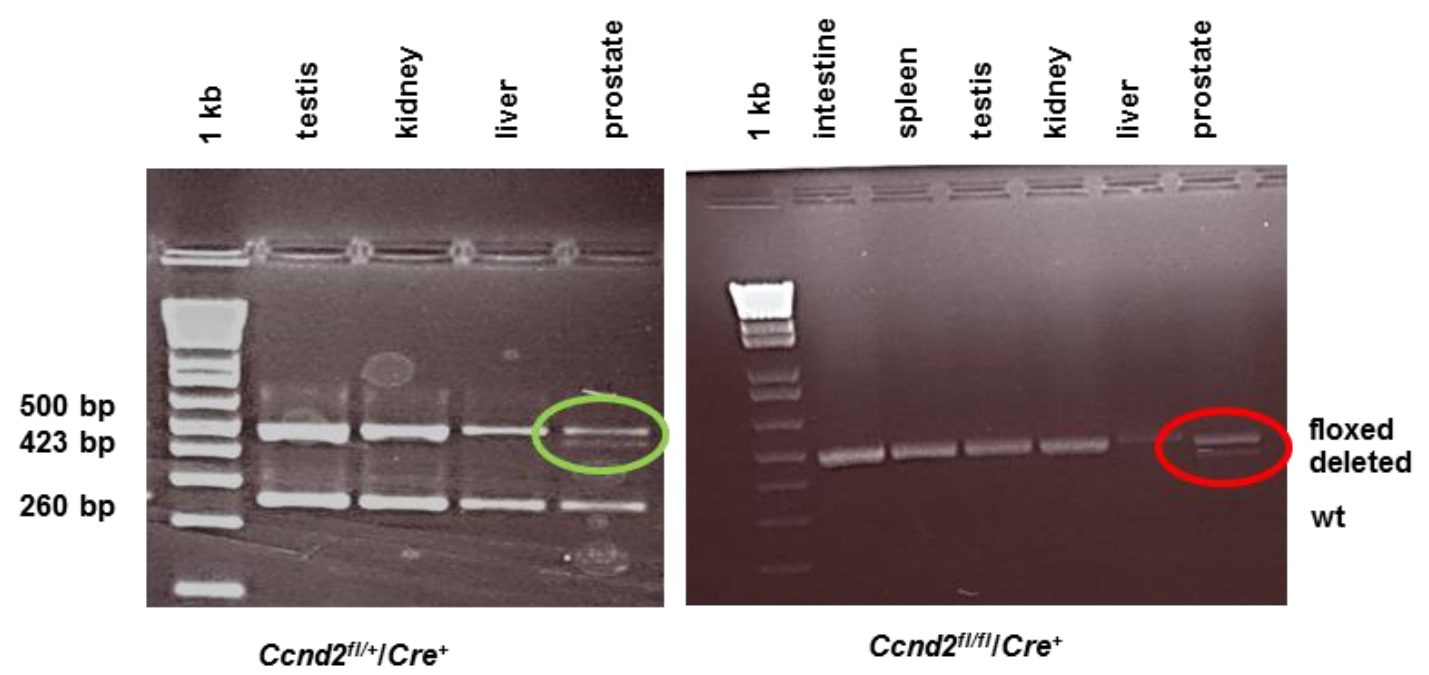

Fig. 3.41: Results of genotyping PCR on tissue DNA isolated from heterozygous and homozygous conditional cyclin D2 knockout mice. Genomic DNA of different tissues, including testis, kidney, liver, intestine, spleen and

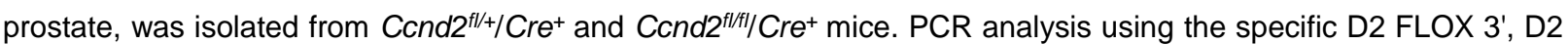
FLOX5' and D2 Delta primers amplified a 500 bp product from the cyclin D2 floxed allele, a 260 bp product from the wild type sequence and a 423 bp product from the knockout allele. The heterozygous $C c n d 2^{f / /} / C r e^{+}$knockout mice showed three bands in the prostate. As expected, the $500 \mathrm{bp}$ band for the floxed allele and the $423 \mathrm{bp}$ band for the deleted allele (green circle) as well as the band for the wild type (wt) allele are visible. The homozygous $\mathrm{Ccnd}^{\mathrm{ff} / \mathrm{fl} /} \mathrm{Cre}^{+}$ knockout mice showed two bands in the prostate (red circle) where only one band for the deleted allele was expected. 1kb: $1 \mathrm{~Kb}$ Plus DNA-ladder

Since by PCR analysis the prostate-specific cyclin D2 knockout could only partially be confirmed, two other methods were applied to show that the generated conditional cyclin D2 knockout mice indeed show a prostate-specific deletion of cyclin D2. On the one hand the expression of cyclin D2 in different tissues of heterozygous and homozygous conditional cyclin D2 knockout mice was tested on the RNA level by quantitative real-time PCR analysis (Fig. 3.42A) and on the other hand on the protein level by western blot analysis (Fig. 3.42B). But again, both methods did not reveal a reduced cyclin D2 expression in prostate tissue of $\mathrm{Ccnd} 2^{f / t / 1} / \mathrm{Cre}^{+}$mice compared to Ccnd $2^{f /+} / \mathrm{Cre}^{+}$mice (Fig. 3.42, red circle). This result was predictable, because also in the present experiment a mixture of prostate cells from the $\mathrm{Ccnd} 2^{f / t / t /} / \mathrm{Cre}^{+}$was analyzed and not pure prostate epithelial cells. 
A

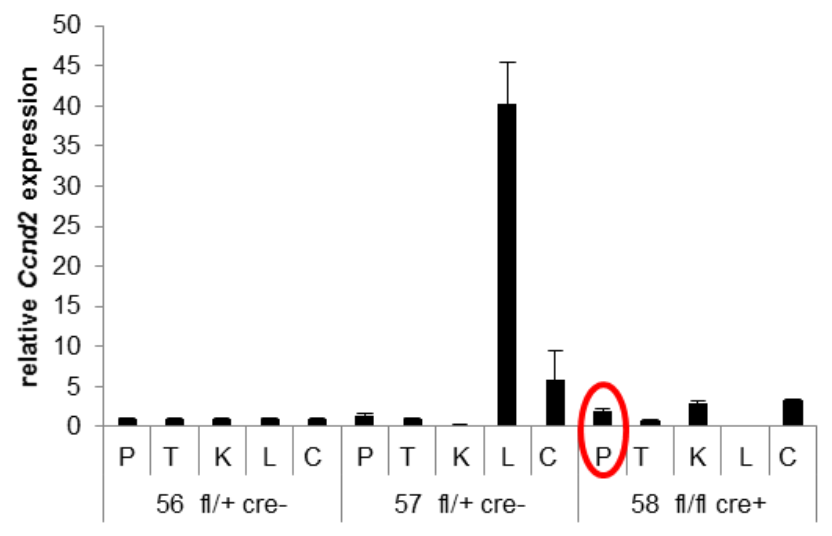

B

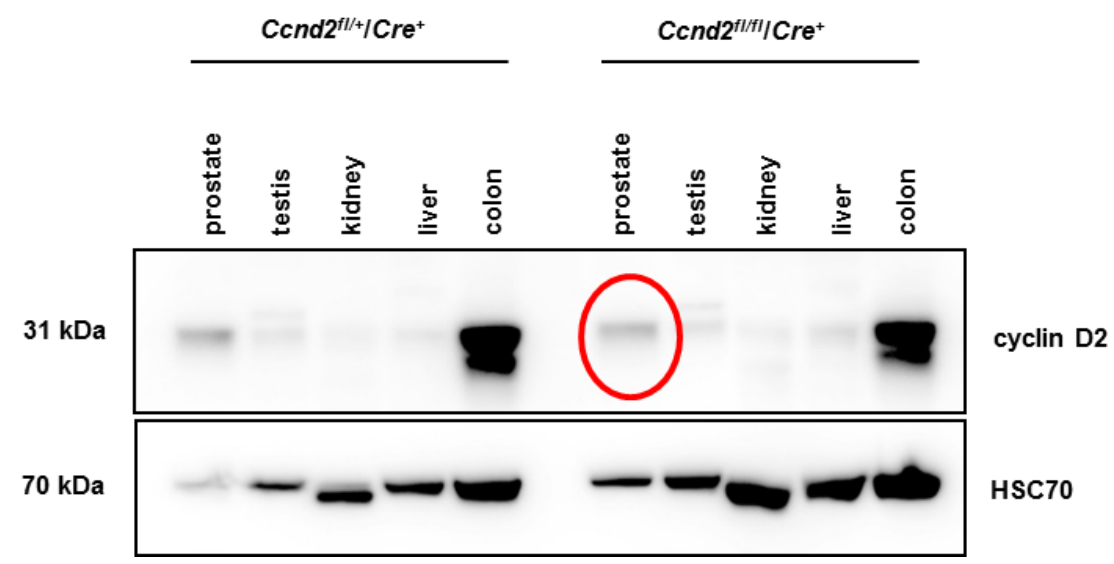

Fig. 3.42: Quantitative real-time PCR and western blot analyses of $\mathrm{Ccnd2}^{\mathrm{fl} /+} / \mathrm{Cre}^{+}$and $\mathrm{Ccnd2}^{\mathrm{fl} / \mathrm{fl} /} / \mathrm{Cre}^{+}$mice. A)

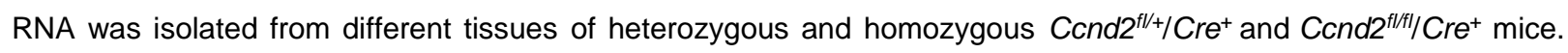
RNA was reverse transcribed into cDNA and the expression of cyclin D2 was analyzed by quantitative real-time PCR. A reduced cyclin D2 expression in the prostate tissue of $C \mathrm{Cnd} 2^{f f / f / /} / \mathrm{Cre}^{+}$mouse as compared to the prostate tissue of $\mathrm{Ccnd} 2^{f /+} / \mathrm{Cre}{ }^{+}$mouse could not be observed. B) Whole protein lysate of different mouse tissues from $\mathrm{Ccnd} 2^{f / /+} / \mathrm{Cre}^{+}$

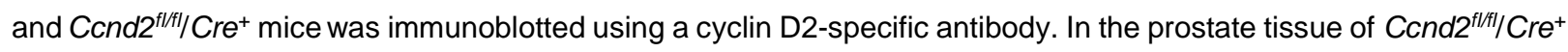
mice cyclin D2 expression was still detectable (red circle) and similarly expressed as compared to prostate tissue of heterozygous Ccnd2//t/Cre+ mice. Immunoblotting of HSC70 ensured equal protein loading. Data are presented as mean + - standard deviation of three quantitative real-time PCR analyses performed in triplicate. P: prostate, T: testis, K: kidney, L: liver, C: colon

Immunohistochemistry was also applied to verify the prostate-specific cyclin D2 knockout in epithelial cells of the prostate from $\mathrm{Ccnd}^{\mathrm{ft} / \mathrm{f} / \mathrm{Cre}} \mathrm{Cr}^{+}$mice. Therefore paraffin sections of the prostate of $C c n d 2^{f / f / f /} / \mathrm{Cre}^{+}$mice were stained with a cyclin D2-specific antibody. Due to the fact that the cyclin D2-specific antibody used for this experiment showed an unspecific staining pattern, a specified conclusion of the cyclin D2 expression could not be drawn from the immunohistochemistry data (data not shown). Because the verification of the prostate-specific 
cyclin D2 knockout in Ccnd2/f/fl/Cre+ mice could only partially be confirmed by PCR analysis, but not quantitative real-time PCR studies as well as western blot analyses, respectively, these methods are not appropriate to detect a cyclin D2 conditional knockout. Therefore, a still ongoing approach is to verify the cyclin D2 conditional knockout in prostate epithelial cells of $\mathrm{Ccnd} 2^{f / / f l} / \mathrm{Cre}^{+}$mice on the RNA level by in situ hybridization.

\subsubsection{Mating of the putative conditional cyclin D2 knockout mice with TRAMP mice}

To study the influence of cyclin D2 on the development and progression of PCa putative male Ccnd $2^{f / / f l} / \mathrm{Cre}^{+}$were mated with heterozygous TRAMP mice $\left(T^{+}\right)$. Male heterozygous $\mathrm{F} 1$ offspring was mated again to homozygous $C c n d 2^{f / / f l}$ mice to generate TRAMP mice with a homozygous conditional cyclin D2 knockout (Ccnd ${ }^{f / f t /} / \mathrm{Cre}^{+} / T^{+}$, Fig.3.43A). TRAMP mice are an established PCa mouse model in which the oncogene SV40 T/t antigen is specifically expressed in prostate epithelial cells under the control of the rat probasin promoter (Gingrich et al., 1997). The prostatespecific expression of the oncogene results in the development of a prostate adenocarcinoma which is reported to develop around week 18 in TRAMP mice (Gingrich et al., 1997)). If cyclin D2 is expected to display tumor suppressive functions in PCa then it can be presumed that in double transgenic TRAMP mice which lack prostate-specific cyclin D2 expression $\left(\mathrm{Ccnd} 2^{\mathrm{flffl} /} / \mathrm{Cre}^{+} / \mathrm{T}^{+}\right)$the prostate tumor development and progression would be enhanced as compared to the tumor development of single transgenic TRAMP mice.

From the above described mating there is only a minor chance of $12.5 \%$ to receive male TRAMP mice with a prostate epithelium-specific homozygous conditional cyclin D2 knockout. The genotype of the offspring was determined by genotyping PCR using genotyping-specific primers. Genomic DNA was isolated from a mouse tail biopsy and then subjected to three different genotyping PCRs (Fig. 3.43B). Using cyclin D2 floxed-specific primers a 260 bp product from the wild type allele, a 500 bp product from the floxed allele and a 423 bp product from the deleted allele were amplified, respectively. Cre-specific primers amplified an app. 500 bp product from the Cre allele. TRAMP mouse-specific primers amplified a 200 bp product from the TRAMP allele. Animal number 103 displayed the desired $\mathrm{Ccnd}^{f / f f /} / \mathrm{Cre}^{+} / T^{+}$genotype (Fig. 3.43B). 
A

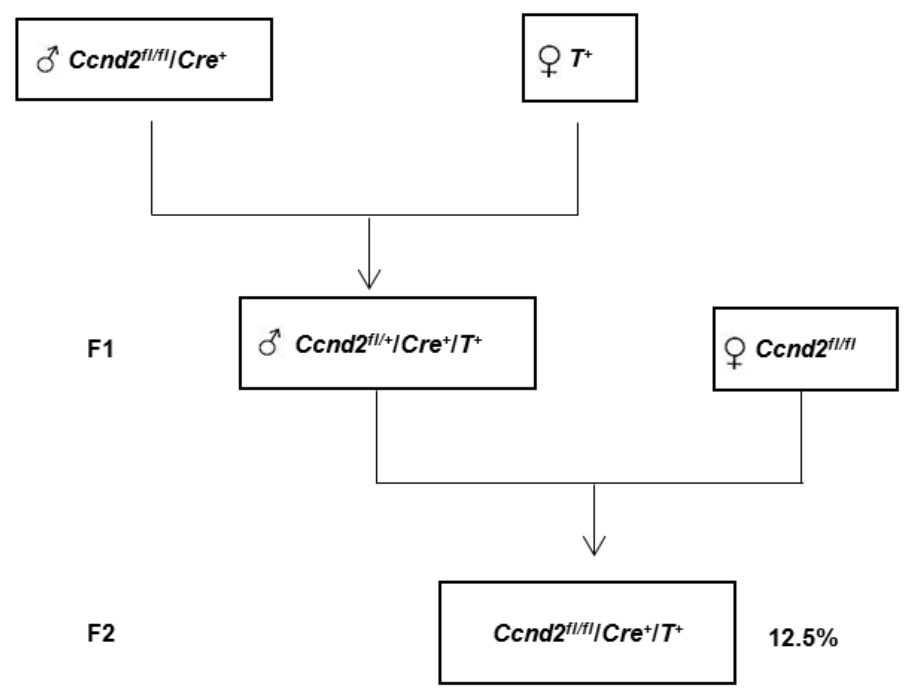

B

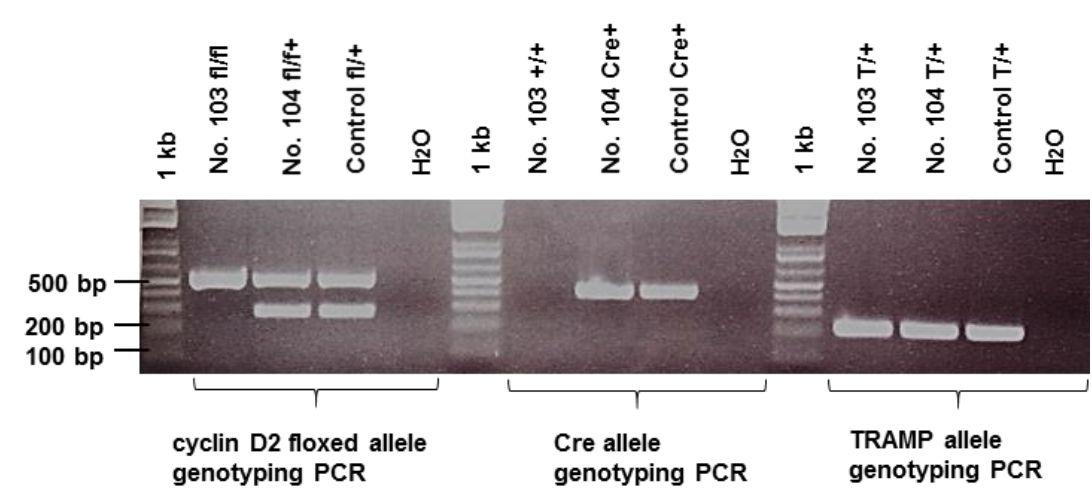

Fig. 3.43: Mating scheme for the generation of TRAMP mice with conditional homozygous cyclin D2 knockout and genotyping PCR. A) Homozygous $\mathrm{Ccnd}^{2 / f / f /} \mathrm{Cre} e^{+}$were mated with heterozygous TRAMP mice $\left(T^{+}\right)$. In the F1 generation heterozygous $\mathrm{Ccnd} 2^{f /+} / \mathrm{Cre}^{+} / T^{+}$mice were generated and further mated to homozygous $\mathrm{Ccnd} 2^{f / / f l}$ mice. There is a $12.5 \%$ chance to receive TRAMP mice with the desired genotype Ccnd2 $/ / / / \mathrm{Cre}^{+} / \mathrm{T}^{+}$in the $\mathrm{F} 2$ generation. B) The genotype of the offspring was determined by three different genotyping PCRs using genotyping-specific primers. Genomic DNA was derived from a tail biopsy. One PCR used cyclin D2 floxed-specific primers and amplified a 260 bp product from the wild type (wt) allele, a 500 bp product from the floxed allele and a 423 bp produced from the deleted allele. The second PCR used Cre-specific primers that amplified an app. 500 bp product from the Cre allele. In the third PCR TRAMP mouse-specific primers were used and a 200 bp product from the TRAMP allele was amplified. A known heterozygous sample as well as $\mathrm{ddH}_{2} \mathrm{O}$ was used as positive and negative controls, respectively. Animal number 103 showed the desired $C \mathrm{Cnd} 2^{\mathrm{fl} / \mathrm{f} / \mathrm{Cre}} / \mathrm{CT}^{+}$genotype. 1kb: $1 \mathrm{~Kb}$ Plus DNA-ladder

Until now, only seven mice with the correct $\mathrm{Ccnd} 2^{f / f t} / \mathrm{Cre}^{+} / \mathrm{T}^{+}$genotype could be obtained, of which the oldest mice is 18 weeks old. These animals are subject to routinely palpation of the prostate in order to determine the onset of tumor development and to study the progression of the prostate tumor. To date, none of the seven $\mathrm{Ccnd} 2^{f / f l} / \mathrm{Cre}^{+} / T^{+}$have developed a PCa. In contrast, three heterozygous $\mathrm{Ccnd} 2^{\mathrm{fl} /+} / \mathrm{Cre}^{+} / \mathrm{T}^{+}$mice had a palpable PCa and had to be sacrificed. 
It turned out that two of these heterozygous $\mathrm{Ccnd} 2^{\mathrm{fl} /+} / \mathrm{Cre}^{+} / T^{+}$mice had seminal vesicle carcinoma and one had a macroscopic PCa. The mouse which had a PCa was just 16 weeks old.

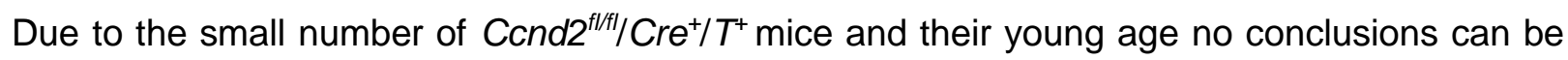
drawn at the moment on the physiological role of cyclin D2 in PCa development and progression. 


\section{Discussion}

\subsection{Summary of results}

One of the two main parts of the present study was the investigation of VPA as a putative candidate for the therapy of $\mathrm{PCa}$. In a previous study, using microarray analysis several candidate genes were identified which were deregulated in mouse 2E PCa cells when treated with VPA (Witt, 2009). These genes included the downregulated genes chemokine (C-X-C motif) ligand 15 (CXC/15), which codes for a cytokine of the CXC chemokine family, RCC1 and BTB domain-containing protein 2 (Rcbtb2) coding for a member of the RCC1-related GEF family, both transcript variants of ceruloplasmin $(C p)$, which is the main copper transporting protein of blood, and leukemia inhibitory factor (Lif), which codes for a class 6 interleukin. Candidate genes that were upregulated after VPA treatment included ubiquitin carboxy-terminal hydrolase L1 (Uchl1), which codes for a de-ubiquitinising enzyme, tyrosine-protein phosphatase-like $N$ (Ptprn), which codes for a member of the protein tyrosine phosphatase family and cyclin D2 (Ccnd2), which codes for a member of the strongly conserved D-type cyclin family (Witt, 2009).

In the present study, the expression of these candidate genes was analyzed by quantitative realtime PCR analyses in prostate tumor tissue and prostate tissue of VPA-treated TRAMP mice. The prostate tumor tissue was derived from an in vivo VPA experiment conducted by Witt during her Ph.D. thesis (Witt, 2012). Since in this first in vivo VPA treatment experiment the tumors were isolated at different time points of age and different treating durations, which could result in differential expression of genes, a new in vivo VPA treatment experiment was conducted during this study. The mice were sacrificed at the age of 16 weeks after 10 weeks of VPA treatment and their prostates were isolated for molecular investigation.

In prostate tumor tissue of VPA-treated TRAMP mice Cp2 and Lif were statistically significant downregulated compared to control-treated mice as revealed by quantitative real-time PCR. Uchl1, Ptprn and Ccnd2 exhibited a different expression pattern to the array result (Witt, 2009). Using western blot analysis the increased Ccnd2 expression after VPA treatment observed in the microarray could be confirmed in prostate tumor tissue.

In prostate tissue derived from the second in vivo VPA treatment experiment, the expression pattern was more similar to the microarray data. The downregulation of $C p 2$ as wells as the upregulation of Ptprn and Uchl1 in prostate tissue of VPA-treated mice compared to prostate tissue of control-treated mice could be confirmed. This indicates that VPA treatment is more effective in the deregulation of the candidate genes at the onset of PCa development. 
In human PCa cells, including LNCaP, DU145 and PC-3, the expression of the candidate gene $C P$ was investigated by quantitative real-time PCR analysis. For all three human PCa cell lines a concentration- and time-dependent decrease in CP expression after VPA treatment could be observed, whereas by western blot analysis the opposite effect was detected.

Since two of the above mentioned candidate genes, namely $C p$ and $C x / 15$, are associated with angiogenesis, a major project of the present study was the investigation of the influence of in vitro and in vivo VPA treatment on tumor- and tumor cell angiogenesis as well as on lymphangiogenesis. Primarily, these studies consisted of expression analyses of angiogenesis and lymphangiogenesis markers. The markers included the angiogenic cytokines vascular endothelial growth factor $A$ and $C$ (Vegfa, VegfC) and angiopoietin 1 (Ang1), three endothelial cell receptor tyrosine kinases (vascular endothelial growth factor receptor 1 (Flt-1/Vegfr1), vascular endothelial growth factor receptor 2 (Kdr/Vegfr2) and tyrosine kinase with immunoglobulin-like and EGF-like domains 1 (Tie-1)), the soluble form of Vegfr2 (sVegfr2) and an endothelial cell adhesion molecule, platelet endothelial cell adhesion molecule (Pecam-1).

In VPA-treated 2E PCa cells almost all angiogenesis markers mentioned above were significantly downregulated in a concentration- and partially time-dependent manner as compared to control cells, except for the two receptors Flt-1/Vegfr1 and Kdr/Vegfr2, which were statistically significant upregulated. By western blot analysis it was shown that the VEGFR2 receptor signaling pathway gets activated after 144 hours of VPA treatment in 2E cells as seen by an increased level of the phosphorylated receptor.

VEGFA expression was also shown to be statistically significant downregulated in human PC-3 PCa cells upon VPA treatment and in prostate tumor tissue of in vivo VPA-treated TRAMP mice. Solely in prostate tissue of VPA-treated TRAMP mice it exhibited a similar expression pattern to controls. Ang1, Tie-1, Kdr/Vegfr2 and Pecam-1 were downregulated in both, prostate tissue and prostate tumor tissue of VPA-treated TRAMP mice. KDR/VEGDR2 expression was increased after VPA treatment in PC-3 cells, similar to 2E cells. FLT-1/VEGFR1 expression was also downregulated in PC-3 cells upon VPA treatment and in prostate tissue of in vivo VPA-treated TRAMP mice, but its expression was unchanged in prostate tumor tissue. The expression of VEGFC and the lymphangiogenesis inhibitor sVEGFR2 in PC-3 cells was dependent on the time of VPA treatment: it increased after 72 hours of VPA treatment but decreased after 144 hours. Taken together, these data confirm that VPA is an alternative treatment option for PCa, mainly because of the induced deregulation of the above mentioned candidate genes associated with $\mathrm{PCa}$ and especially because it exerts anti-angiogenic properties, for both, blood and lymphangiogenesis, which has not been described to date for the latter. 
To confirm the influence of VPA treatment on angiogenesis several chicken chorioallantoic membrane (CAM) experiments were conducted using the human PCa cells PC-3 and the mouse PCa cells 2E. These cells underwent different VPA pre-treatment strategies using different VPA concentrations. Macroscopic analysis of the CAM tumors revealed that tumors could be affected in three different ways: either by bloody spots within the tumor, by bloodshot tumors or by disturbed blood vessel formation. VPA treatment of PC-3 cells resulted in a reduced number of bloodshot tumors in two experiments. The other observations for $2 \mathrm{E}$ and PC-3 cell tumors were not consistent within the experiments for one cell line and also not comparable within the two cell lines.

CAM tumor volume was not significantly altered in tumors derived from VPA-treated cells as compared to controls. Immunofluorescent staining with the lymphatic vessel marker Prox1 and the blood vessel marker Mep21 of untreated tumors showed that $2 \mathrm{E}$ cell tumors grow mainly by blood angiogenesis and PC-3 cell tumors by blood- and lymphangiogenesis. In VPA-treated CAM tumors raised from PC-3 cells treated with 3mM VPA, but without pre-treatment, Prox1 staining was slightly reduced in the distal tumor part.

In CAM tumors derived from PC-3 cells that were pre-treated for 24 hours with 1mM VPA no statistically significant expression differences of the tested angiogenesis markers (VEGFA, VEGFC, ANG1, FLT-1/VEGFR1, KDR/VEGFR2, sVEGFR2, PECAM-1, TIE-1 and CP) could be observed as compared to controls. In CAM tumors derived from $2 \mathrm{E}$ cells that were pre-treated for 24 hours with $1 \mathrm{mM}$ VPA only Cp 1 was statistically significant downregulated. The other tested genes were not significantly deregulated except VEGFA which showed a tendency to an upregulated expression. CAM tumors derived from $2 \mathrm{E}$ cells which were pre-treated for 1 week with 3mM VPA exhibited increased expression of Ccnd2 and Vegfa. Taken together, the CAM assay could only partially confirm the anti-angiogenic features of VPA in PCa.

The second major project of the present thesis was the investigation of the role of the candidate gene cyclin D2 as a putative tumor suppressor gene in PCa.

Human LNCaP and PC-3 PCa cell clones with stable overexpression of cyclin D2 were generated. The incorporation of the expression vector was confirmed by the presence of about 100\% EGFP-positive cells. Cyclin D2 overexpression was confirmed by immunofluorescent staining and western blot analysis. LNCaP colonies with a doxycycline-inducible cyclin D2 overexpression were also generated and the overexpression was verified by western blot experiments.

Human PCa cells with a transient, stable or inducible cyclin D2 overexpression had a similar or slightly increased proliferation rate as compared to controls. Surprisingly, both PC-3 and LNCaP 
cell clones with stable cyclin D2 overexpression had an increased migration rate as compared to controls.

$\mathrm{NIH} / 3 \mathrm{~T} 3$ cells with endogenous cyclin D2 expression were transfected with cyclin D2-specific siRNAs and showed an increased migration rate compared to luciferase control transfected cells, whereas cells transfected with a cyclin D1-specific siRNA had a similar migration behavior as compared to control cells. Cyclin D2 siRNA transfected NIH/3T3 cells had acquired no transformation potential, which was investigated by soft agar assay.

Since it was shown that siRNA transfection is not effective over a longer time period NIH/3T3 clones with doxycycline-inducible cyclin D2 downregulation were generated. Their proliferation rate was similar to that of control cells not treated with doxycycline. Besides, they also acquired no transformation potential, not even when double transfected with the oncogene KRAS. This can be explained by the fact, that the transfection efficiency was very low.

Contrary to the expectation, the functional consequence of siRNA-mediated cyclin D2 downregulation in VPA-treated 2E, LNCaP, PC-3 and DU145 cells was a reduced proliferation rate.

To elucidate the physiological role of cyclin D2 in the organism a conditional cyclin D2 knockout mouse model was generated. The purchased $C c n d 2^{f / f l}$ and $P B-C r e 4^{+}$mouse lines were established and bred to generate homozygous $\mathrm{Ccnd} 2^{f / f / t} / \mathrm{Cre}^{+}$mice. The knockout was partially confirmed by PCR analysis of prostate tissue, but could not be confirmed by quantitative realtime PCR and western blot analyses. To definitely verify the prostate-specific deletion of cyclin D2 other experiments, including RNA in situ hybridization, are still ongoing.

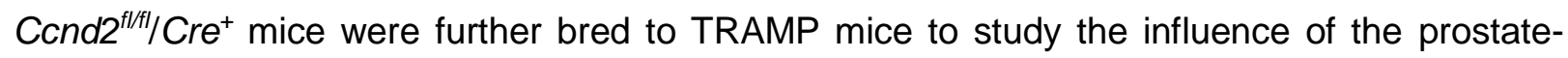
specific cyclin D2 deletion on prostate tumor development and progression. To date, there are seven homozygous $\mathrm{Ccnd} 2^{\mathrm{f} / \mathrm{fl} /} / \mathrm{Cre}^{+} / \mathrm{T}^{+}$mice, which are too young to have even developed a PCa yet. Therefore, no conclusions can be drawn at the moment on the physiological role of cyclin D2 in the mouse prostate.

\subsection{The histone deacetylase inhibitor VPA}

Valproic acid is a histone deacetylase inhibitor (HDI) that selectively inhibits the catalytic activity of class I histone deacetylates (HDACs) and induces proteasomal degradation of class II histone deacetylates (Göttlicher et al. 2001, Krämer et al. 2003). Histones are the primary protein components of chromatin, which compact the DNA. The chromatin structure can exist in two states, either acetylated or de-acetylated. Acetylated chromatin is structurally loose and strongly de-condensed to allow binding of RNA and DNA polymerases to transcribe and replicate the 
DNA. HDACs catalyze the deacetylation of histones in chromatin, making it strongly condensed and transcriptionally inactive. Condensed chromatin can be transformed again into the more relaxed, de-condensed structure by histone acetyltransferases (HATs, Gillet et al. 2007). Treatment with HDIs, such as VPA, inhibits the activity of HDACs, thereby increasing the level of acetylated chromatin since HATs are still active, resulting in increased gene expression.

VPA was licensed in Europe in 1960 and in the United States in 1978 for the treatment of epilepsy. Now it is widely available throughout the world and became the drug of choice in primary generalized epilepsies. In the mid-1990s, VPA was approved for treatment of partial seizures. Its potential is exerted in the human brain, where VPA acts on the neurotransmitter Gamma-Aminobutyric acid (GABA) by potentiating the inhibitory activity of GABA in several ways, for example by inhibition of GABA degradation, increased synthesis of GABA and decreased GABA turnover (Johannessen 2000, Johannessen and Johannessen 2003, Owens and Nemeroff 2003, Kostrouchová et al. 2007).

This makes VPA a long known drug which has been extensively studied and its side effects are well known. Common clinical side effects of VPA include dyspepsia, weight gain, dysphoria, fatigue, dizziness, drowsiness, hair loss, headache, nausea, sedation and tremor. VPA can also impair liver function and prolong blood coagulation times. Although very rare, side effects such as spina bifida and other defects of neural tube closure are observed when used in early pregnancy, indicating that valproic acid interferes with the developmental regulatory pathway (Lammer et al. 1987, Koren and Kennedy 1999, Koren et al. 2006, Kostrouchová et al. 2007). HDls important role in regulating transcription makes them also very promising drugs for the therapy of cancer. Not only did studies using neuroblastoma-, glioma- and promyelotic leukemia cells lines reveal a promising proliferation inhibition after VPA treatment (Fischkoff and Walter 1984, Regan 1985) but also studies on several solid tumors, including cancer of the bladder (Ozawa et al. 2010, Vallo et al. 2011, Byler et al. 2012), hepatocellular cancer (Machado et al. 2011), head and neck cancer (Gan et al. 2012), pancreatic cancer and colon cancer (Jones et al. 2008, Venkataramani et al. 2010), renal cell carcinoma (Jones et al. 2009), small cell lung cancer (Hubaux et al. 2010), cervical cancer (Sami et al. 2008) and breast cancer (Fortunati et al. 2008). VPA treatment alone proved to be beneficial but also in combination with other anticancer therapeutics, like cytotoxic agents among others, which exhibited synergistic anti-cancer effects (summarized in Sun and Coy, 2014). To date, VPA is already commonly prescribed for brain tumor patients (glioma, Zhou et al. 2014) and suberoylanilide hydroxamic acid (SAHA, also known as Vorinostat), another HDAC inhibitor, is approved to treat patients with cutaneous $\mathrm{T}$ cell lymphoma (Edelstein et al. 2009). 
In PCa, several in vitro studies using VPA also resulted in proliferation inhibition (Xia et al. 2006, Annicotte et al. 2006, Shabbeer et al. 2007, Gao et al. 2007, Chou et al. 2011, 2015). In vivo studies using PC-3-, DU145-, LNCaP- or C4-2-Z xenografts demonstrated a reduced tumor volume after VPA treatment (Xia et al. 2006, Shabbeer et al. 2007, Gao et al. 2007, Angelucci et al. 2008). Thereby, proliferation inhibition observed in in vitro and in vivo studies was due to functional effects of VPA treatment inducing either growth arrest, cell death, senescence or antiangiogenic properties (Shabbeer et al. 2007, Gao et al. 2007, Wedel et al. 2011). Although, the underlying molecular mechanism for the VPA-induced proliferation inhibition remain unsolved. Shabbeer et al. (2007) reported an increase in p21 expression, but this is a general effect not only observed in cancer cells but also in non-malignant cells. Besides, Brinkmann et al. (2001) could show that after HDI treatment with suberic bishydroxamate (SBHA) the expression of p21 was upregulated in human fibroblast cells, whereas the proliferation rate was not affected (Brinkmann et al. 2001).

Thus, the molecular mechanism for the VPA-induced proliferation inhibition in the tumor further remained unresolved and was subject to investigation during the present study. Besides, the in vivo VPA treatment studies conducted so far were carried out with xenografted mice instead of a PCa mouse model, thereby the natural situation will not be resembled. In the present study, in vivo VPA treatment was conducted with the Transgenic Adenocarcinoma of the Mouse Prostate (TRAMP) PCa mouse model and its molecular mechanisms were analyzed on prostate and prostate tumor tissue of these mice. The TRAMP mouse is one of the first PCa models and one of the most widely used mouse model in PCa research since it represents the course of the human disease (Fig 4.1). Using this model, conclusions on tumor initiation and progression can also be drawn and not only on tumor growth like in conventional xenograft models (MentorMarcel et al. 2001). The transgene of TRAMP mouse model comprises the minimal probasin promoter $(-426 /+28,426$ basepairs of the rat probasin (PB) gene promoter and 28 basepairs of 5'-untranslated region driving viral SV40 large-T and small t antigens which results in prostate epithelium-specific inactivation of the two tumor suppressors $p R b$ and $p 53$ (Murphree and Benedict 1984, Greenberg et al. 1994, Gingrich et al. 1996, Surget et al. 2013). Thus, TRAMP mice will develop prostatic intraepithelial neoplasia (PIN) by 8 weeks which will progress to high grade PIN by 18 weeks and poorly differentiated and invasive adenocarcinoma by 28 weeks of age. With nearly $100 \%$ of penetrance at the age of 40 weeks TRAMP mice have developed an adenocarcinoma (Fig. 4.1, Gingrich et al. 1997, Mentor-Marcel et al. 2001). The TRAMP mouse model also displays metastasis to distant organs, including the lung and lymph nodes (Gingrich et al. 1997), although rarely to the bone which in human PCa is the major side for metastasis. The majority of PCa patients die due to the development of a metastatic disease of which $80 \%$ 
is primarily localized in the bone (Coleman 2006, Ganguly et al. 2014). 5-12\% of prostate cancer patients with clinically organ-confined cancer exhibit regional lymph node metastasis (Cai et al. 2009). More rarely, prostate cancer metastases can occur in the adrenal gland, lung, liver and brain.

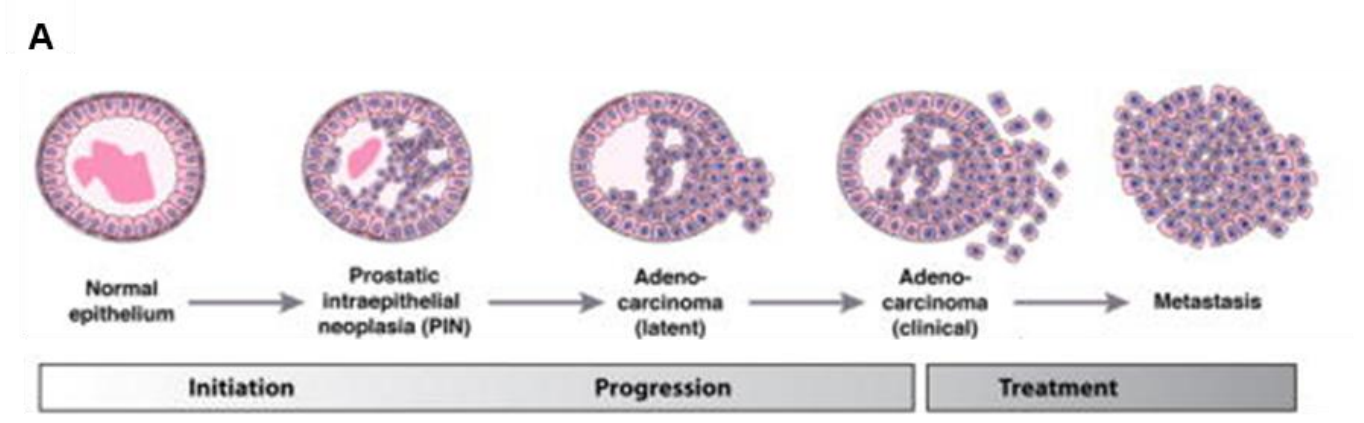

B

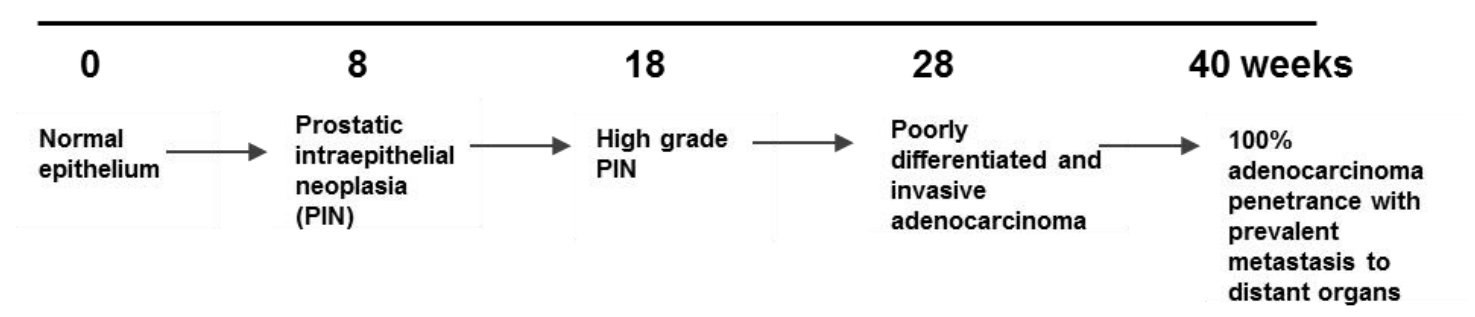

Fig. 4.1: Schematic presentation of PCa progression in humans and in the TRAMP mouse. A) The course of the human PCa disease is depicted. Normal prostatic epithelium develops into prostatic intraepithelial neoplasia (PIN) which progresses into a latent adenocarcinoma and a clinically relevant, castration resistant adenocarcinoma which metastasizes preferably to the bone. B) The timeline represents the course of PCa development and progression in the TRAMP mouse model. Starting after puberty at the age of 8 weeks TRAMP mice develop PIN which progresses into high grade PIN by 18 weeks and into poorly differentiated and invasive adenocarcinoma by 28 weeks. With nearly $100 \%$ penetrance TRAMP mice develop an adenocarcinoma at the age of 40 weeks which also displays prevalent metastasis to distant organs, although rarely to the bone. The TRAMP mouse model reproduces the course of the mouse PCa disease and is therefore suitable for studies on PCa initiation, progression and treatment. Modified from Irshad and Abate-Shen 2013.

The positive effects observed during in vitro and in vivo VPA treatment studies prompted the initiation of several clinical trials investigating the effect of VPA treatment in hematological tumor diseases (Wagner et al., 2010), breast cancer, lung cancer, pancreatic cancer, cervical cancer, testicular and ovarian cancer (Arce et al. 2006, Candelaria et al. 2007, Munster et al. 2009, Chateauvieux et al. 2010), as well as in glioblastoma (Weller et al. 2011) and melanoma (Rocca et al. 2009). Generally, patients responded well to VPA treatment, which was partially combined with chemo- and radiotherapy. 
To date, only a single phase 2 study was conducted with PCa patients that received oral VPA treatment. Merely 10 patients were included in this study of which one died due to PCa progression. Two patients exhibited reduced PSA levels and no tumor progression (Sharma et al. 2008) but due to toxicity this trial had to be discontinued early.

In male patients that received long term antiepileptic treatment with VPA, it was shown that they exhibited lower age-correlated PSA levels as compared to control groups. VPA treatment also exerted anti-proliferative effects on neoplastic PCa cells in vitro (LNCaP) and in vivo (Stettner et al. 2012), further highlighting the beneficial effect of VPA treatment in PCa patients.

\subsubsection{Deregulated candidate genes after VPA treatment of mouse 2E PCa cells}

In order to investigate the molecular mechanism underlying the VPA-induced inhibition of proliferation described in section 4.2 the expression of previously identified candidate genes (Witt, 2009) was analyzed in prostate and prostate tumor tissue of in vivo VPA-treated TRAMP mice. Witt et al. (2013) could show that treatment of murine PCa 2E cells with VPA resulted not only in inhibition of proliferation, but also in inhibition of migration and invasion. These functional effects were concentration- and time-dependent. Microarray analysis of $2 \mathrm{E}$ cells treated for 24 hours with VPA identified several genes that were deregulated after VPA treatment (Witt, 2009). Eight candidate genes were chosen for further investigation because of their known expression in the prostate and their connection to cancer (Table 4.1). Their deregulation in $2 \mathrm{E}$ cells was confirmed by quantitative real-time PCR. Thereby it was shown that VPA treatment had a concentration- and time-dependent effect on the expression of these candidate genes (Witt et al., 2013, summarized in Table 4.1). 
Table 4.1: Deregulated candidate genes in murine 2E PCa cells after 24 hours of VPA treatment (Witt et al. 2013).

\begin{tabular}{|c|c|c|}
\hline $\begin{array}{c}\text { Candidate } \\
\text { gene }\end{array}$ & Connection to cancer/PCa & $\begin{array}{l}\text { Expression } \\
\text { in murine 2E } \\
\text { PCa cells } \\
\text { after VPA } \\
\text { treatment }\end{array}$ \\
\hline Cxcl15 & $\begin{array}{l}\text { belongs to the CXC chemokine family of which members containing an } \\
\text { ELR-(Glu-Leu-Arg-) motif can induce angiogenic activity (Strieter et al. } \\
\text { 1995) } \\
\text { in multiple myeloma patients the plasma level of these chemokines was } \\
\text { reported to be increased (Pappa et al. 2011) }\end{array}$ & \\
\hline Rcbtb2 & 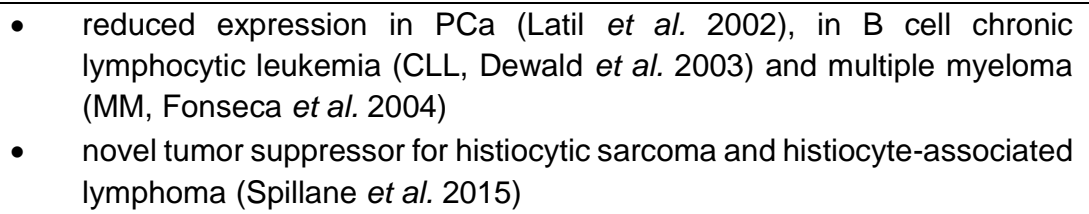 & \\
\hline $\begin{array}{l}\text { Cp1 } \\
\text { Cp2 }\end{array}$ & $\begin{array}{l}\text { - } \quad \text { transcription is dependent on high copper concentration as well as } \\
\text { Hif1a initiation (Martin et al. 2005) } \\
\text { PCa patients have a significantly increased plasma level of CP (Nayak } \\
\text { et al. 2003) and serum CP was even suggested as a marker for PCa } \\
\text { (Fotiou et al. 2007) }\end{array}$ & \\
\hline Lif & $\begin{array}{l}\text { - undergoes a functional transition from paracrine growth inhibitor to } \\
\text { autocrine growth stimulator during progression of PCa to the hormone- } \\
\text { refractory phenotype (Chung et al. 1999) } \\
\text { stimulates breast and kidney cancer cell proliferation (Kellokumpu- } \\
\text { Lehtinen et al. 1996) }\end{array}$ & \\
\hline Uchl1 & 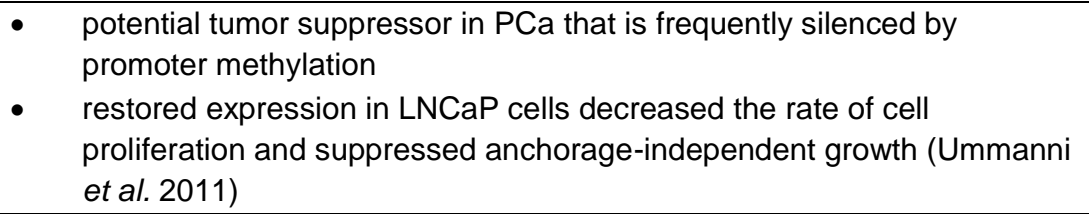 & \\
\hline Ptprn & $\begin{array}{l}\text { - } \text { Ptprf decreased survival in PCa when overexpressed (DaSilva et al. } \\
\text { 2013, Du and Grandis 2015) } \\
\text { Ptprn is overexpressed in gastrointestinal neuroendocrine carcinomas } \\
\text { (Leja et al. 2008) }\end{array}$ & \\
\hline Ccnd2 & $\begin{array}{l}\text { - abnormal expression of D-type cyclins and CDKs is linked to cancer } \\
\text { development and progression (Musgrove et al. 2011) } \\
\text { in PCa, the CCND2 promoter is frequently hypermethylated, which is } \\
\text { associated with loss of mRNA expression and tumor development } \\
\text { (Henrique et al. 2006) }\end{array}$ & \\
\hline
\end{tabular}

To date, the analyses on the effect of VPA treatment on PCa were solely conducted with xenografted mice, thereby the natural situation is not resembled like in a PCa mouse model, such as the TRAMP model which is a more similar model to the human situation. Witt et al. (2013) could show that in vitro VPA treatment also exerts effects on PCa progression in the murine PCa 2E cells, which were derived from a PCa of a TRAMP mouse. This raised the question of whether the in vitro observed effects are replicable in vivo. Therefore, in the present study the expression of the above mentioned candidate genes was investigated by quantitative 
real-time PCR and western blot analyses in prostate tumor tissue and prostate tissue of VPAand control-treated TRAMP mice.

\subsubsection{Candidate gene expression in prostate tumor tissue and prostate tissue of in vivo VPA-treated TRAMP mice}

In vivo studies are essential for research on a putative new therapeutic agent. For the treatment of PCa some VPA in vivo studies have already been conducted. Thereby it was shown that treatment of mice with $0.4 \% \mathrm{w} / \mathrm{v}$ VPA over the drinking water was well tolerated and resulted in a constant plasma level of 0.4mM VPA (Shabbeer et al. 2007).

The first in vivo VPA treatment experiment in our research group was conducted by D. Witt (Dissertation, 2012). She used three different TRAMP mice study groups that were treated with a final concentration of $0.4 \% \mathrm{w} / \mathrm{v}$ VPA and sweetener over the drinking water. Control-treated mice received drinking water with sweetener only. Two of the study groups received preventive VPA treatment at the age of 6 weeks when the prostate shows no malignant changes yet. Of these, one group was treated until the age of 30 weeks, when tumor progression should still be ongoing, and then sacrificed. The second group was treated until the age of 40 weeks, when TRAMP mice should have developed a PCa, and then sacrificed. The third group received VPA as a curative treatment starting at the age of 16 weeks, when tumor development should have initiated already in TRAMP mice, and at the age of 40 weeks they were sacrificed (Mentor-Marcel et al., 2001). Prostate tumors were isolated for molecular evaluation and subjected to quantitative real-time PCR analysis to confirm in vivo the deregulation of the chosen candidate genes after VPA treatment (Table 4.2).

In prostate tumor tissue of VPA-treated TRAMP mice the expression of Cp2 was statistically significant reduced as demonstrated by quantitative real-time PCR analysis, which is in accordance to the previously obtained array data (Table 4.2). The expression of the other candidate genes was also decreased, although not statistically significant. Rcbtb2 expression was comparable to controls. By densitometrical evaluation of a western blot the upregulation of CCND2 after VPA treatment could also be confirmed in prostate tumor tissue of VPA-treated TRAMP mice as compared to controls (Table 4.2).

Quantitative real-time PCR of prostate tumor tissue of in vivo VPA-treated TRAMP mice solely confirmed the downregulation of $C p 1, C p 2$ and Lif as observed after in vitro VPA treatment of 2E cells (Table 4.2). 
Table 4.2: Summary of the results concerning candidate gene expression in 2E cells after VPA treatment and in prostate tumor tissue of VPA-treated TRAMP mice. Expression patterns that differ from those observed in VPAtreated $2 \mathrm{E}$ cells are marked with a red arrow. The ones that are similar expressed after in vitro and in vivo VPA treatment are marked with a gray arrow. An unchanged expression pattern as compared to untreated controls is marked with a blue arrow. n.d: not determined

\begin{tabular}{|c|c|c|}
\hline Candidate gene & $\begin{array}{c}\text { Expression in murine 2E PCa cells } \\
\text { after VPA treatment }\end{array}$ & $\begin{array}{c}\text { Expression in prostate tumor } \\
\text { tissue of VPA-treated TRAMP } \\
\text { mice }\end{array}$ \\
\hline Cxcl15 & - & n.d. \\
\hline Rcbtb2 & $\checkmark$ & \\
\hline Cp1 & $\checkmark$ & \\
\hline Cp2 & $\checkmark$ & \\
\hline Lif & $\checkmark$ & real-time PCR: \\
\hline Ptprn & $\checkmark$ & western blot: \\
\hline Ccnd2 & $\checkmark$ & \\
\hline
\end{tabular}

Several aspects can be responsible for the poor correlation of the candidate gene expression profile observed after in vitro and in vivo VPA treatment.

Since no non-pathologic prostate tissue of in vivo VPA-treated TRAMP mice was isolated for expression comparison, it cannot be excluded that the few $\mathrm{PCa}$ tumors may have risen due to failure of therapy or resistance to VPA treatment. Besides, tumors were isolated at different time points depending on which of the three study groups the mice belonged to. To date, resistance to in vivo or in vitro VPA treatment has not yet been described for PCa. But, it is known that VPA induces irreversible resistance in human colon cancer cells (Fedier et al. 2007) and in renal cell carcinoma in vivo (Juengel et al. 2013). In the latter case resistance is associated with elevated AKT activation (Juengel et al. 2013).

The difference in the mice age, when their prostate tumor was isolated, could also lead to differential expression of the candidate genes. This initiated implementation of a new in vivo VPA treatment experiment of TRAMP mice in the present study, in which the mice were sacrificed at the same age, namely at 16 weeks of age (see section 2.19.1). The prostates were isolated for molecular evaluation by quantitative real-time PCR analysis to confirm the in vitro observed deregulation of the candidate genes in vivo (Table 4.3). 
In prostate tissue of VPA-treated TRAMP mice the downregulation of Cp2 as well as the upregulation of Uchl1 and Ptprn after VPA treatment could be confirmed. Rcbtb2 was not quite statistically significant upregulated, which is the only contrary result to the array data. The other genes displayed an unchanged expression pattern as compared to the controls (Table 4.3).

Table 4.3: Summary of the results concerning candidate gene expression in 2E cells after VPA treatment, in prostate tumor tissue of VPA-treated TRAMP mice and in prostate tissue of VPA-treated TRAMP mice. Expression patterns that differ from those observed in VPA-treated 2E cells are marked with a red arrow. The ones that are similar expressed after in vitro and in vivo VPA treatment are marked with a gray arrow. An unchanged expression patterns as compared to untreated controls is marked with a blue arrow. n.d.: not determined

\begin{tabular}{|c|c|c|c|}
\hline Candidate gene & $\begin{array}{l}\text { Expression in murine } \\
\text { 2E PCa cells after VPA } \\
\text { treatment }\end{array}$ & $\begin{array}{l}\text { Expression in prostate } \\
\text { tumor tissue of VPA- } \\
\text { treated TRAMP mice }\end{array}$ & $\begin{array}{l}\text { Expression in prostate } \\
\text { tissue of VPA-treated } \\
\text { TRAMP mice }\end{array}$ \\
\hline Cxcl15 & & n.d. & \\
\hline \multicolumn{4}{|l|}{ Rcbtb2 } \\
\hline \multicolumn{4}{|l|}{ Cp1 } \\
\hline \multicolumn{4}{|l|}{ Cp2 } \\
\hline \multicolumn{4}{|l|}{ Lif } \\
\hline \multicolumn{4}{|l|}{ Uchl1 } \\
\hline \multicolumn{4}{|l|}{ Ptprn } \\
\hline Ccnd2 & & $\begin{array}{l}\text { real-time: } \\
\text { western blot: }\end{array}$ & \\
\hline
\end{tabular}

Expression analysis of prostate tissue from the second in vivo VPA treatment experiment confirmed the deregulation of more candidate genes, including Cp1, Cp2, Uchl1 and Ptprn, as compared to the expression analysis of prostate tumor tissue from the first in vivo VPA treatment. More genes had a similar expression pattern as compared to controls, but only one gene, namely Rcbtb2, exhibited a contrary expression pattern to the previously obtained array data (Witt, 2009). This indicates that VPA treatment has already a clear influence on the expression of genes when adenocarcinomas have not yet developed. It has to be mentioned that it was not possible to evaluate the analyzed prostates histopathologically. Due to the small size of the prostate gland, the complete tissue was used for RNA isolation. Therefore, it cannot be excluded that in some TRAMP mice the development of prostatic intraepithelial neoplasia (PIN) had already taken place. 
Taken together, molecular effects observed by in vitro VPA treatment of mouse PCa cells (2E) could be partially confirmed in prostate tumor tissue of in vivo VPA-treated TRAMP mice and to a greater extend in prostate tissue of in vivo VPA-treated TRAMP mice. In the latter, the only contrary result as compared to the in vitro studies was the observed upregulation of Rcbtb2 expression in prostate tissue of VPA-treated mice. Interestingly, Rcbtb2 is a known tumor suppressor whose expression is frequently lost or downregulated in PCa (Latil et al. 2002). Therefore, increased Rcbtb2 expression upon VPA treatment should be favorable for PCa patients, nonetheless. In conclusion it can be stated, that in vitro and in vivo VPA treatment influences the expression of $\mathrm{PCa}$-associated genes positively, which might inhibit or reduce tumor progression. These results further highlight the beneficial effects of VPA as an alternative treatment opportunity for PCa.

One candidate gene, namely ceruloplasmin $(C p)$, was chosen for further, more profound studies due to its clinical relevance in PCa. PCa patients have a significantly increased plasma level of ceruloplasmin (Nayak et al. 2003) and serum ceruloplasmin was even suggested as a marker for PCa (Fotiou et al. 2007). Ceruloplasmin, the main copper carrier in blood, is known to be indirectly involved in angiogenesis, but only when copper is bound (Raju et al. 1982). Its transcription is initiated by high copper concentrations and hypoxia-inducible factor-1a (Hif1 $\alpha$ ) activation (Martin et al. 2005). Hypoxic conditions favor angiogenesis as the heterodimeric transcription factor HIF-1 induces the expression of vascular endothelial growth factors (Vegfs), the main angiogenic cytokines (Banerjee et al. 2007).

VPA treatment of mouse $2 \mathrm{E}$ PCa cells resulted in a statistically significant decreased $C p$ expression in a concentration- and time-dependent manner, accompanied with reduced Vegfa expression and increased Hif1 $\alpha$ expression (Witt, 2012). In the present study it was confirmed by quantitative real-time PCR that the expression of human CP also mostly decreased in a timeand concentration-dependent manner in human PCa cells after VPA treatment. In contrast, by western blot analysis the downregulation of CP after VPA treatment of human PCa cells could not be confirmed. Since no consistent results of ceruloplasmin expression after VPA treatment in human PCa cells could be observed, studies on ceruloplasmin were discontinued.

\subsection{Tumor angiogenesis in general}

Angiogenesis, the formation of new blood vessels from existing blood vessels, was identified as one of the 'hallmarks of cancer' by Hanahan and Weinberg $(2000 ; 2011)$ due to the recognition that this process is of crucial importance during the transition from benign hyperplastic nodules to malignant lesions (Folkman, 1971). The importance of angiogenesis for tumor growth and 
metastasis was also shown in 1982 by Muthukkaruppan et al. who infused cancer cells in the iris, which is supplied with blood circulation, and into the anterior chamber of the eye, which is not supplied with circulation. The cancer cells without blood circulation grew to 1-2 $\mathrm{mm}^{3}$ and then stopped. When placed in an area where angiogenesis was possible they grew beyond 2 $\mathrm{mm}^{3}$ (Muthukkaruppan et al. 1982). It was even shown that without vascular support, tumors may become necrotic or even apoptotic (Holmgren et al. 1995, Parangi et al. 1996).

Angiogenesis is indispensable for tumor growth and metastatic spread, since without adequate supply of oxygen and nutrients and the removal of waste products the tumor could not grow past a few millimeters in diameter (Fig. 4.2). Not only blood vessels but also lymphatic vessels are thereby essential. New lymphatic vessels grow by a process called lymphangiogenesis.

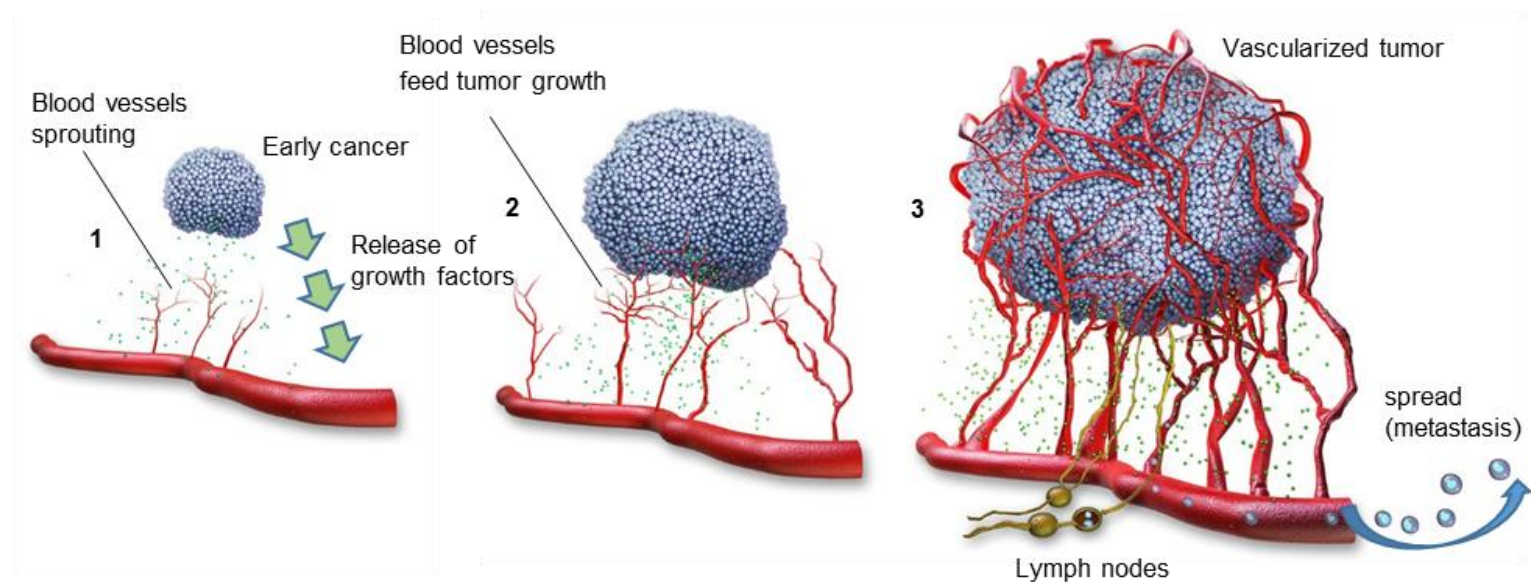

Fig. 4.2: Process of tumor angiogenesis. When the tumor requires oxygens and nutrients for further growth the angiogenic switch shifts the balance of angiogenic activators and inhibitors to the activators. 1) The tumor produces growth factors, such as VEGF, in response to gene mutation or hypoxia and releases them. 2) Growth factors bind to endothelial cell receptors thereby inducing signal transduction pathways and causing cell proliferation for the generation of new blood and lymphatic vessels. 3) Once cancer cells penetrate blood or lymphatic vessels, they receive oxygen and nutrients for further growth and they are able to remove waste products. Cancer cells are also able to circulate through the intravascular stream and then proliferate at another site by formation of metastases. Modified from the Angiogenesis Foundation, http://www.dianasaville.com, 17.01.2016.

Tumors remain dormant in an avascular state at diameters of 1 to $2 \mathrm{~mm}$ (Folkman 1974) and proceed to a vascular state when the angiogenic switch is turned on. Further growth is possible only after new capillaries form a network to supply the tumor with oxygen, nutrients and immune cells (Folkman 1971). Once cancer cells penetrate blood or lymphatic vessels they circulate through the intravascular stream and then proliferate at another site by formation of metastases (Folkman 1971). Angiogenesis is regulated by the balance of angiogenic activators and inhibitors. When the tumor requires fuel, the angiogenic switch shifts the balance of angiogenic activators and inhibitors to the activators. Their expression will be upregulated and 
simultaneously the expression of the inhibitors or negative regulators will be decreased (Dameron et al. 1994). Pro-angiogenic factors will be released from the tumor to stimulate angiogenesis and lymphangiogenesis. It has been shown that expression levels of angiogenic factors reflect the aggressiveness of tumor cells (Weidner et al. 1993).

The process of neovascularization, which is the formation of new vessels in tissue not normally containing them, including tumor angiogenesis, can be categorized into ten steps. During the first step angiogenic factors produced in the tumor will be released, which then during the second step bind to endothelial cell receptors located on a vessel, thereby inducing an intracellular signaling cascade. In the third step the capillary basement membrane will be enzymatically degraded, resulting in hypoxia. During the fourth step, endothelial cells activated by angiogenic factors proliferate and then during the fifth step migrate directional. In the sixth step the extracellular matrix will be remodeled so that during the seventh step the tube can be formed. During the eighth step a loop for circulation is formed. The ninth step encompasses stabilization of the vessel by smooth muscle cells or pericytes and finally the tenth step includes the continuous stimulation of the angiogenic process by angiogenic factors since vascular endothelial cells divide only about every 1000 days on average (Denekamp 1993, Nishida et al. 2006, Adair and Montani 2010).

Naturally occurring proteins that are able to inhibit angiogenesis include angiostatin, endostatin, interferon, platelet factor 4, thorombospondin, prolactin $16 \mathrm{kd}$ fragment and tissue inhibitor of metalloproteinase-1, -2 and -3 (Nishida et al. 2006).

The main angiogenic activators, of which more than a dozen have been identified, include growth factors such as vascular endothelial growth factor (VEGF), basic fibroblast growth factor (bFGF), angiogenin, transforming growth factor (TGF)- $\alpha$, TGF- $\beta$, tumor necrosis factor (TNF)- $\alpha$, plateletderived endothelial growth factor, hepatocyte growth factor, epidermal growth factor, granulocyte colony-stimulating factor and placental growth factor, cytokines like interleukin-8, and other endogenous modulators like angiopoetin-1 (Ang-1, Nishida et al. 2006, Kluetz et al. 2010, Mukherji et al. 2013).

The angiogenic activators of the VEGF family and their receptors (VEGFRs, depicted in Fig. 4.3) are receiving the most attention since VEGF is a powerful angiogenic agent in neoplastic tissues, as well as in normal tissues. Among the VEGF family, VEGF-A, VEGF-B and VEGF-C are known to induce proliferation of blood vessels when acting on their respective receptors, while VEGF$\mathrm{C}$ and VEGFD are involved in lymphangiogenesis when binding to their respective receptors (Neufeld et al. 1999, Mandriota et al. 2001, Pepper 2001, Rafii and Skobe 2003).

VEGF-A is overexpressed in a variety of tumors (Dvorak 2002) and it is the most studied of all the angiogenic activators. It is a potent and very specific mitogen for vascular endothelial cells 
that stimulates the full cascade of events required for angiogenesis (Leung et al. 1989, Conn et al. 1990, Nishida et al. 2006). VEGF expression can be triggered for example by hypoxia, resulting from the increasing distance between the growing tumor cells and the capillaries or from the inefficiency of new vessels. This results in induction of VEGF expression and its receptors via Hif-1a (Bottaro and Liotta 2003).

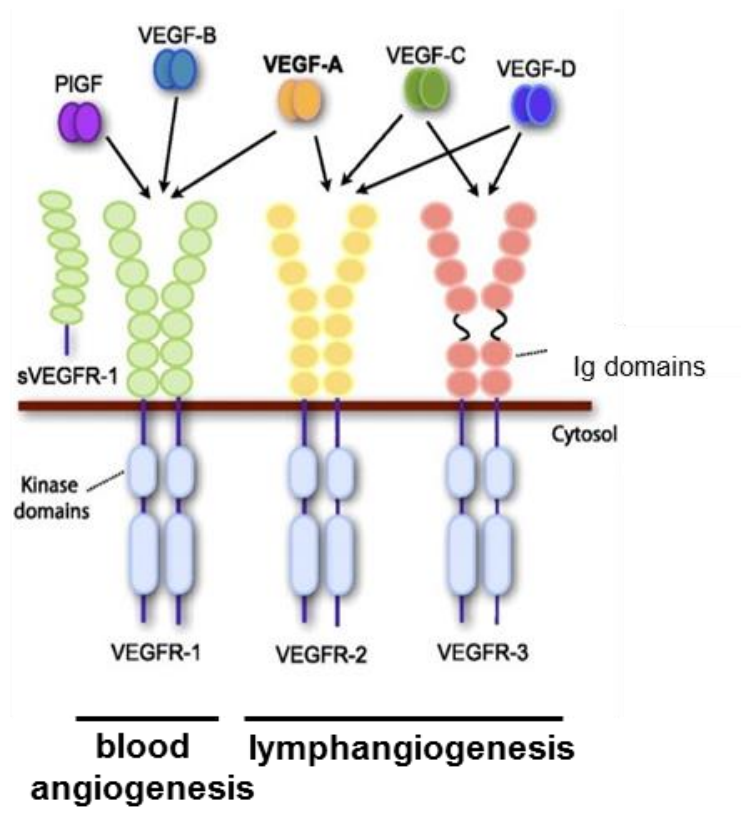

Fig. 4.3: VEGF family members and their receptors. The mammalian VEGF family members include VEGF-A, -B, -C, -D, and placental growth factor (PIGF). VEGF-A can bind to VEGFR-1 and VEGFR-2. VEGF-B and PIGF only bind to VEGFR-1. VEGF-A, VEGF-B, VEGF-C induce proliferation of blood vessels when acting on their respective receptors. VEGF-C and -D bind to VEGFR-3 and VEGFR-2, thereby promoting blood- and lymphangiogenesis. Modified from Almodovar et al. 2009.

Activation of endothelial cells by VEGF results in production of matrix metalloproteinases (MMPs) which are responsible for the breakdown of the extracellular matrix filling the space between cells. Matrix breakdown permits the migration of endothelial cells into the surrounding tissue. There they organize into hollow tubes which finally evolve into a mature blood vessel network. Platelet endothelial cell adhesion molecule (PECAM-1) participates in adhesive and signaling procedures required for the motility of endothelial cells and their subsequent organization into vascular tubes (DeLisser et al. 1997, Cao et al. 2002) as well as other adhesion factors like integrin $\alpha$ or $\beta$, which are also indispensable for this process (Mizejewski 1999, Nelson et al. 2000). Newly formed blood vessels are stabilized and governed to vascular growth by the angiogenic factor angiopoietin-1 (Ang-1) and its receptor Tie-2. Angiopoietin-2 (Ang-2) is a naturally occurring antagonist for Ang-1 and Tie-2 competing for Tie-2 binding (Suri et al. 1996, Maisonpierre et al. 1997, Tournaire et al. 2004). It is known to disrupt blood vessel formation 
(Hu and Cheng 2009). Additionally to ligand-binding, Tie-2 forms a pre-formed hetero-oligomeric complex with the related receptor tyrosine kinase Tie-1 by physical interaction (Marron et al. 2000, Chen-Konak et al. 2003). The role of Tie-1 in angiogenesis is not fully clear since the Tie1 ectodomain is unable to bind angiopoietins (Davis et al. 1996). The Tie-2:Tie-1 complex seems to partially block Ang-1 binding to Tie-2 (Marron et al. 2007). In response to VEGF, inflammatory stimuli or changes in shear stress the Tie-1 ectodomain can undergo regulated cleavage whereby it is removed. This has been shown to increase access of Ang-1 to Tie-2 resulting in enhanced Ang-1-activation of Tie-2 and Tie-2-mediated signaling (Marron et al. 2007, Hansen et al. 2010).

It has been observed that all VEGF ligands and receptors, except for VEGFR-2, show extensive staining in human prostate specimens. In epithelial cells, VEGF-A and VEGFR-1 expression was higher in tumor tissue as compared to benign tissue whereas VEGF-D and VEGFR-3 expression was significantly higher in benign tissue as compared to tumors in the stroma and the endothelium of lymphatic and blood vessels. Additionally, the incidence of lymphatic vessels, but not blood vessels, was lower in tumor tissue as compared with benign tissue. These observations suggest that activation of VEGFR-1 by VEGF-A within the tumor, and activation of lymphatic endothelial cell receptor VEGFR-3 by VEGF-D within the adjacent benign stroma may be important signaling mechanisms involved in the progression and subsequent metastatic spread of $\mathrm{PCa}$. Consequently, inhibition of these pathways may contribute to therapeutic strategies for the management of PCa (Woollard et al. 2013).

\subsubsection{Antiangiogenic treatment in cancer}

Since angiogenesis is essential for tumor growth and metastasis, controlling tumor-associated angiogenesis has been shown to inhibit tumor progression (Ferrara and Kerbel 2005). This makes anti-angiogenic treatment is an emerging, promising approach for the treatment of cancer.

There are several ways one can inhibit angiogenesis: inhibition of pro-angiogenic factors such as VEGF, for example by use of an antibody, inhibition of receptors for pro-angiogenic factors (VEGF-receptors), by inhibiting endothelial cells, inhibition of proteases (inhibit the synthesis of MMP), by interrupting the signaling pathways, by raising the concentration of anti-angiogenic factors or directly by killing tumor-related vascular endothelial cells (Kluetz et al. 2010, Hwang and Heath 2010).

Anti-angiogenic agents have proven to be effective in the treatment of several solid tumors, including renal cell carcinoma (Motzer et al. 2007), colorectal cancer (Hurwitz et al. 2004) and 
non-small cell lung cancer (Sandler et al. 2006). Bevacizumab for example, an anti-VEGF antibody, which has been shown to be effective in multiple cancer cell lines, is currently Food and Drug Administration- (FDA) approved for treatment of several malignancies including colorectal carcinoma (Cohen et al. 2007a), non-squamous non-small cell lung cancer (Cohen et al. 2007b), metastatic breast cancer (Montero et al. 2012), recurrent glioblastoma (Chamberlain 2011) and most recently metastatic renal cell carcinoma (Pichler, 2015).

Angiogenesis plays an important role in the pathophysiology of PCa as several studies correlate markers of angiogenesis with metastatic disease, higher Gleason score and clinical outcome (Kluetz et al. 2010). Weidner et al. could show that tumor angiogenesis, measured by microvessel density (MVD), correlates with metastasis (Weidner et al. 1993). Besides, MVD of tumor samples at diagnosis was statistically significant correlated with stage, grade and diseasespecific survival, as revealed by a study following PCa patients for a median of 15 years (Borre et al. 1998). Plasma levels of VEGFs are increased in patients with metastatic PCa (Duque et al. 1999) making them an independent prognostic factor in men with metastatic PCa (George et al. 2001, El-Gohary et al. 2007). Lastly, HIF-1a, a key mediator of VEGF expression, was shown to have a higher expression in PCa than in benign prostate tissue (Du et al. 2003). Concluding from these findings, angiogenesis inhibition could be a powerful strategy to treat PCa.

Surprisingly, clinical trials for angiogenesis-inhibition conducted with PCa patients have so far not been as successful as expected. A selected number of clinical trials of anti-angiogenic agents in development for PCa and their results/status are demonstrated in Table 4.4. The tested antiangiogenic agents can be grouped into different classes of which one class consists of antiVEGF antibodies, like bevacizumab, another class consists of immunomodulators like lenalidomide, thalidomide and tasqunimod and the biggest class consists of tyrosine-kinaseinhibitors (TKIs) like sunitinib, sorafenib, cabozantinib, vandetanib, pazopanib and cediranib. Other agents tested include an mTor inhibitor (temsirolimus), a VEGF trap (aflibercept), an antiendoglin antibody (TCR105) and an anti-angiopoietin (AMG386, Table 4.4). Most of the studies are still ongoing and the ones already finished had mostly negative results. For example, a large phase III study in castration-resistant prostate cancer (CRPC) with bevacizumab in combination with docetaxel chemotherapy did not improve overall survival in men and was even associated with greater toxicity (Kelly et al. 2012). Combination of docetaxel with other anti-angiogenic agents also did not increase the overall survival or progression-free survival. Contrary, it might even increase the risk for treatment-related mortality (Lei et al. 2014). Clinical phase III trials using the anti-angiogenic agents sunitinib or lenalidomide on CRPC patients were even discontinued due to futility (Mukherji et al. 2013). Targeting the dual receptors VEGFR2/MET with the tyrosine kinase inhibitor cabozantinib was slightly more effective as improvements in 
bone scans and pain response in PCa patients were observed (Lee and Smith 2013, Smith et al. 2013).

In general, the role of anti-angiogenic treatment in PCa has yet to be defined. Although no angiogenesis-inhibitors have been approved for patients with metastatic CRPC, the rationale for the use of anti-angiogenic therapy in CRPC remains strong nonetheless and novel antiangiogenic agents are being constantly investigated (Bilusic and Wong 2014).

Table 4.4: Selected clinical trials of anti-angiogenic agents in development for PCa therapy. This table shows selected clinical trials of anti-angiogenic agents in development for the therapy of PCa. The tested anti-angiogenic agent, its target, the study with its primary end point and the results or status of the study are displayed. mCRPC: metastatic castration-resistant prostate cancer, PDGFR: platelet derived growth factor receptor; EGFR: epidermal growth factor receptor; mAb: monoclonal antibody; OS: overall survival; PFS: Progression free survival; mTOR: mammalian target of rapamycin; TTP: time to progression. Modified from Mukherji et al. 2013.

\begin{tabular}{|c|c|c|c|c|}
\hline Drug & Class & Study & $\begin{array}{l}\text { Primary } \\
\text { end point }\end{array}$ & Result/Status \\
\hline Bevacizumab & VEGF mAb & $\begin{array}{l}\text { Phase III chemotherapy-naïve } \\
\text { mCRPC: docetaxel/prednisone } \\
\text { +/- bevacizumab }\end{array}$ & OS & $\begin{array}{l}\text { OS } 22.6 \mathrm{~m} \text { vs. } 21.6 \\
\mathrm{~m}(p=0.181), \text { median } \\
\text { PFS } 9.9 \mathrm{~m} \text { vs. } 7.5 \mathrm{~m} \\
\text { ( } p<0.001) . \text { Increasec } \\
\text { toxicity with addition } \\
\text { of bevacizumab }\end{array}$ \\
\hline $\begin{array}{l}\text { 1. Bevacizumab } \\
\text { 2. Temsirolimus }\end{array}$ & $\begin{array}{l}\text { 1. VEGF mAb } \\
\text { 2. mTor inhibitor }\end{array}$ & $\begin{array}{l}\text { Phase I/II docetaxel-refractory } \\
\text { mCRPC: bevacizumab + } \\
\text { temsirolimus }\end{array}$ & $\begin{array}{l}\text { Objective } \\
\text { response }\end{array}$ & ongoing \\
\hline Aflibercept & VEGF trap & $\begin{array}{l}\text { Phase III chemotherapy naïve } \\
\text { mCRPC: docetaxel/prednisone } \\
\text { +/- aflibercept }\end{array}$ & OS & $\begin{array}{l}\text { Negative (full results } \\
\text { awaited) }\end{array}$ \\
\hline $\begin{array}{l}\text { 1. Thalidomide } \\
\text { 2. Bevacizumab }\end{array}$ & $\begin{array}{l}\text { 1. Immuno- } \\
\text { modulator } \\
\text { 2. VEGF mAb }\end{array}$ & $\begin{array}{l}\text { Phase II chemotherapy naïve } \\
\text { mCRPC: docetaxel/prednisone+ } \\
\text { bevacizumab+ thalidomide }\end{array}$ & $\begin{array}{l}\text { PSA decline } \\
>50 \%\end{array}$ & $\begin{array}{l}\text { PSA response rate: } \\
90 \% \text {, median TTP } \\
18.3 \mathrm{~m} \text {, median OS } \\
28.2 \mathrm{~m} \text {, all patients } \\
\text { grade } 3 / 4 \text { neutropenia }\end{array}$ \\
\hline Lenalidomide & $\begin{array}{l}\text { Immuno- } \\
\text { modulator }\end{array}$ & $\begin{array}{l}\text { Phase III mCRPC, docetaxel +/- } \\
\text { lenalidomide }\end{array}$ & OS & $\begin{array}{l}\text { Negative (full results } \\
\text { awaited) }\end{array}$ \\
\hline Sunitinib & $\begin{array}{l}\text { TKI (VEGFR/ } \\
\text { PDGFR) }\end{array}$ & $\begin{array}{l}\text { Phase III mCRPC after } \\
\text { progression on docetaxel: } \\
\text { sunitinib + prednisone vs. } \\
\text { placebo + prednisone }\end{array}$ & OS & $\begin{array}{l}\text { Prematurely } \\
\text { discontinued due to } \\
\text { futility }\end{array}$ \\
\hline Sorafenib & $\begin{array}{l}\text { TKI (VEGFR/ } \\
\text { PDGFR) }\end{array}$ & $\begin{array}{l}\text { Phase II pre-docetaxel mCRPC: } \\
\text { sorafenib + docetaxel }\end{array}$ & $\begin{array}{l}\text { PSA decline } \\
>50 \%\end{array}$ & ongoing \\
\hline Cabozantinib & $\begin{array}{l}\text { TKI (VEGFR2/ } \\
\text { MET) }\end{array}$ & $\begin{array}{l}\text { 1. Non-randomized expansion } \\
\text { cohort (phase II) CRPC post- } \\
\text { docetaxel: cabozantinib single } \\
\text { agent } \\
\text { 2. Phase III CRPC post- } \\
\text { docetaxel, post- } \\
\text { abiraterone/enzalutamide: } \\
\text { cabozantinib vs. prednisone } \\
\text { 3. Phase III cabozantinib vs. } \\
\text { mitoxantrone in previously } \\
\text { treated CRPC }\end{array}$ & $\begin{array}{l}\text { Bone scan } \\
\text { response } \\
\text { rate } \\
\text { OS } \\
\text { Pain } \\
\text { response }\end{array}$ & $\begin{array}{l}60 \% \text { bone scan } \\
\text { response rate } \\
\text { ongoing } \\
\text { ongoing }\end{array}$ \\
\hline Vandetanib & $\begin{array}{l}\text { TKI (VEGFR2/ } \\
\text { EGFR) }\end{array}$ & $\begin{array}{l}\text { Phase II mCRPC pre-docetaxel: } \\
\text { vandetanib + bicalutamide vs. } \\
\text { bicalutamide }\end{array}$ & $\begin{array}{l}\text { PSA } \\
\text { response }\end{array}$ & ongoing \\
\hline
\end{tabular}




\begin{tabular}{|l|l|l|l|l|}
\hline Pazopanib & $\begin{array}{l}\text { TKI (VEGFR/ } \\
\text { PDGFR) }\end{array}$ & $\begin{array}{l}\text { Phase I/II mCRPC pre- } \\
\text { docetaxel: docetaxel+/- } \\
\text { pazopanib }\end{array}$ & PFS & ongoing \\
\hline Cediranib & TKI (VEGFR) & $\begin{array}{l}\text { Phase II mCRPC pre-docetaxel: } \\
\text { docetaxel +/- cediranib }\end{array}$ & PFS & ongoing \\
\hline Tasquinimod & $\begin{array}{l}\text { Immuno- } \\
\text { modulator }\end{array}$ & $\begin{array}{l}\text { 1. Phase II mCRPC: } \\
\text { tasquinimod vs placebo } \\
\text { 2. Phase III randomized } \\
\text { mCRPC: tasquinimod vs. } \\
\text { placebo }\end{array}$ & PFS & $\begin{array}{l}7.6 \mathrm{~m} \text { vs. 3.3 m } \\
(p=0.0042) \text { for } \\
\text { median PFS } \\
\text { ongoing }\end{array}$ \\
\hline TCR105 & $\begin{array}{l}\text { mAb against CD- } \\
105 \text { (endoglin) }\end{array}$ & Phase II mCRPC & PFS & ongoing \\
\hline AMG386 & Anti-angiopoietin & $\begin{array}{l}\text { Phase II mCRPC: AMG386 +/- } \\
\text { abiraterone }\end{array}$ & PFS & Ongoing \\
\hline
\end{tabular}

\subsubsection{VPA as a possible anti-angiogenic treatment}

Since Witt et al. (2013) could observe a VPA-induced effect on the regulation of angiogenesisassociated genes, namely $C p$ and $C x c / 15$ (described in 4.2.1), the influence of in vitro and in vivo VPA treatment on angiogenesis in PCa was subject to further investigation during the present study.

To date, only little is known about the effects of VPA treatment on angiogenesis. VPA is known to up-regulate the anti-angiogenic proteins thrombospondin-1 and activin A (Cinatl et al. 2002). In vitro studies on human umbilical vein endothelial cells and in vivo studies in the chicken chorioallantoic membrane assay (CAM) and in a Matrigel plug assay showed that VPA inhibits angiogenesis in vitro and in vivo (Michaelis et al. 2004). In vitro VPA treatment inhibited proliferation, migration and tube formation. In vivo VPA treatment, as investigated by matrigel plug assay, showed that few distinct and perfused vessels were formed after VPA treatment. Additionally, the CAM assay showed reduced vessel ingrowth, the development of irregular and brittle vessels, and a markedly reduced perfusion as compared with control CAM (Michaelis et al. 2004).

In terms of a VPA-induced effect on angiogenesis in PCa, Witt et al. (2013) could show that the expression of $C p$ and $C x c / 15$, two genes associated with angiogenesis, decreased in a timeand concentration-dependent manner in mouse PCa $2 \mathrm{E}$ cells after VPA treatment. This was accompanied by reduced Vegfa expression and increased Hif-1 $\alpha$ expression. Besides from that, a study by Shabbeer et al. (2007) could show that angiogenesis was inhibited after VPA treatment $(0.4 \% \mathrm{w} / \mathrm{v})$ of PC-3 xenografted mice, as measured by microvessel density after CD31 staining. However, inhibition of angiogenesis was only observed in PC-3 xenografts, but not in DU145 xenografts. 
Another study by Gao et al. (2007) showed that PC-3 cell xenografts which were treated with VPA had a reduced expression of vascular endothelial growth factor, the main angiogenic stimulator.

Since very little is known about the molecular mechanisms underlying the VPA-mediated antiangiogenic properties in $\mathrm{PCa}$, they were further elucidated in the present project by in vitro and in vivo studies. As mentioned above, solely one in vitro study shows that VPA treatment exerts an effect on the expression on angiogenesis markers in PCa cells (Witt et al. 2013). Besides, to date only two in vivo studies demonstrated that VPA treatment displayed an inhibitory effect on tumor angiogenesis in $\mathrm{PCa}$, which were both conducted with xenograft mice (Shabbeer et al. 2007, Gao et al. 2007). In the present study, a PCa mouse model was used, namely the TRAMP mouse model, resembling the human PCa superiorly as compared to a xenograft model.

Additionally, for the in vitro studies a suitable cell line was used, namely the $2 \mathrm{E}$ cell line, which was generated from a PCa of a TRAMP mouse (Hardenberg, 2010).

The expression of several angiogenesis markers (Fig. 4.4), including Vegfa, Vegfc, Flt-1/Vegfr1, Kdr/Vegfr2, sVegfr2, Ang-1, Tie-2 and Pecam-1 was analyzed by quantitative real-time PCR after VPA treatment of mouse PCa cells 2E and human PCa cells PC-3.

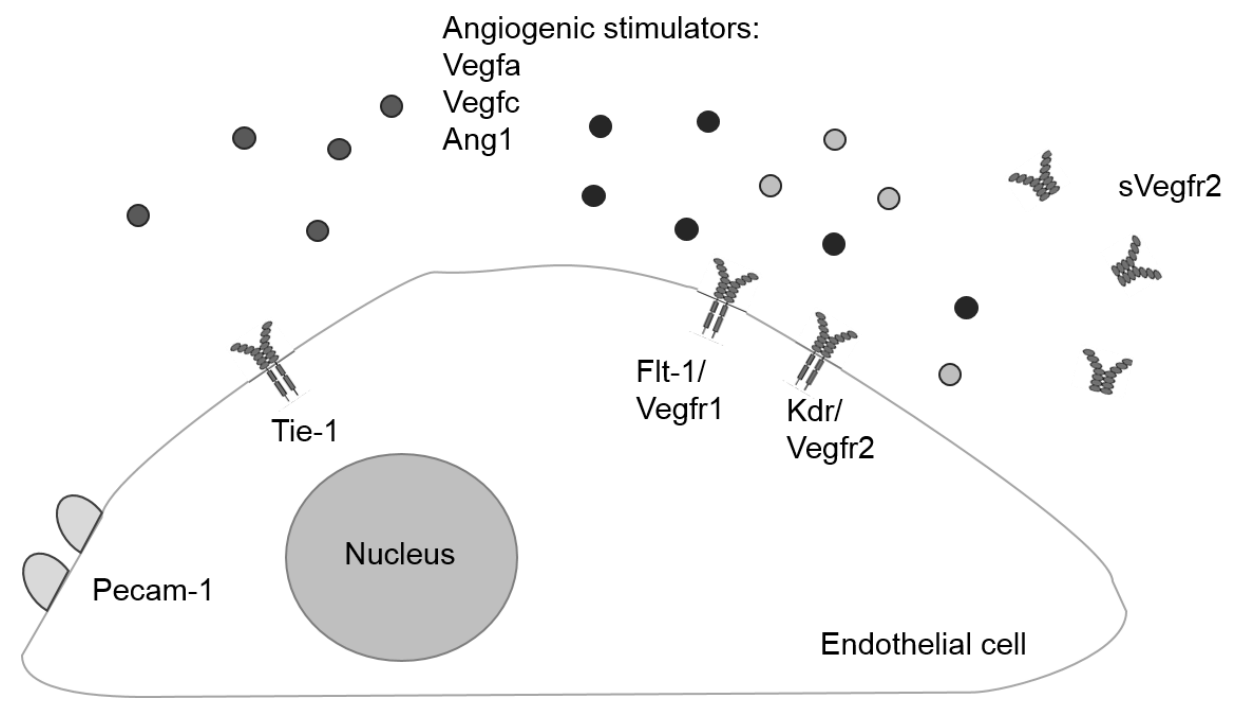

Fig. 4.4: Angiogenesis markers used for expression analyses of PCa cells, prostate tissue and prostate tumor tissue after in vitro and in vivo VPA treatment. The markers used for expression analyses included the angiogenic cytokines vascular endothelial growth factor $A$ and $C$ (Vegfa, VegfC) and angiopoietin 1 (Ang1), three endothelial cell receptor tyrosine kinases (vascular endothelial growth factor receptor-1 (Flt-1/Vegfr1), vascular endothelial growth factor receptor-2 (Kdr/Vegfr2) and tyrosine kinase with immunoglobulin-like and EGF-like domains 1 (Tie-1)), the soluble form of Vegfr2 (sVegfr2) and an endothelial cell adhesion molecule, platelet endothelial cell adhesion molecule (Pecam-1). Black, dark and light grey circles represent the angiogenic stimulators Vegfa, Vegfc and Ang1. 
Analyzing the expression of the above mentioned angiogenesis markers in PCa cells or tissue after in vitro or in vivo VPA treatment is was expected to be reduced compared to controls in order to exert its anti-angiogenic properties. Indeed, it could be observed in $2 \mathrm{E}$ cells that upon VPA treatment the expression of Vegfa, Vegfc and Ang1 as well as Tie-1 and Pecam-1 was statistically significant downregulated, mostly in a time- and concentration- dependent manner. However, the two receptor tyrosine kinases Flt-1/Vegfr1 and Kdr/Vegfr2 were upregulated after VPA treatment (Fig.4.5). In PC-3 cells, VEGFA and FLT-1/VEGFR1 were also downregulated after VPA treatment, depending on the duration of VPA treatment. In contrast, KDR/VEGFR2 was upregulated. VEGFC and sVEGFR expression, dependent on the duration of VPA treatment, were upregulated after 72 hours, but downregulated after 144 hours (Fig.4.5). sVEGFR2, an alternatively spliced variant of VEGFR2, is known to inhibit developmental and reparative lymphangiogenesis by blocking VEGFC function while competing for VEGFR2 binding (Albuquerque et al. 2009).

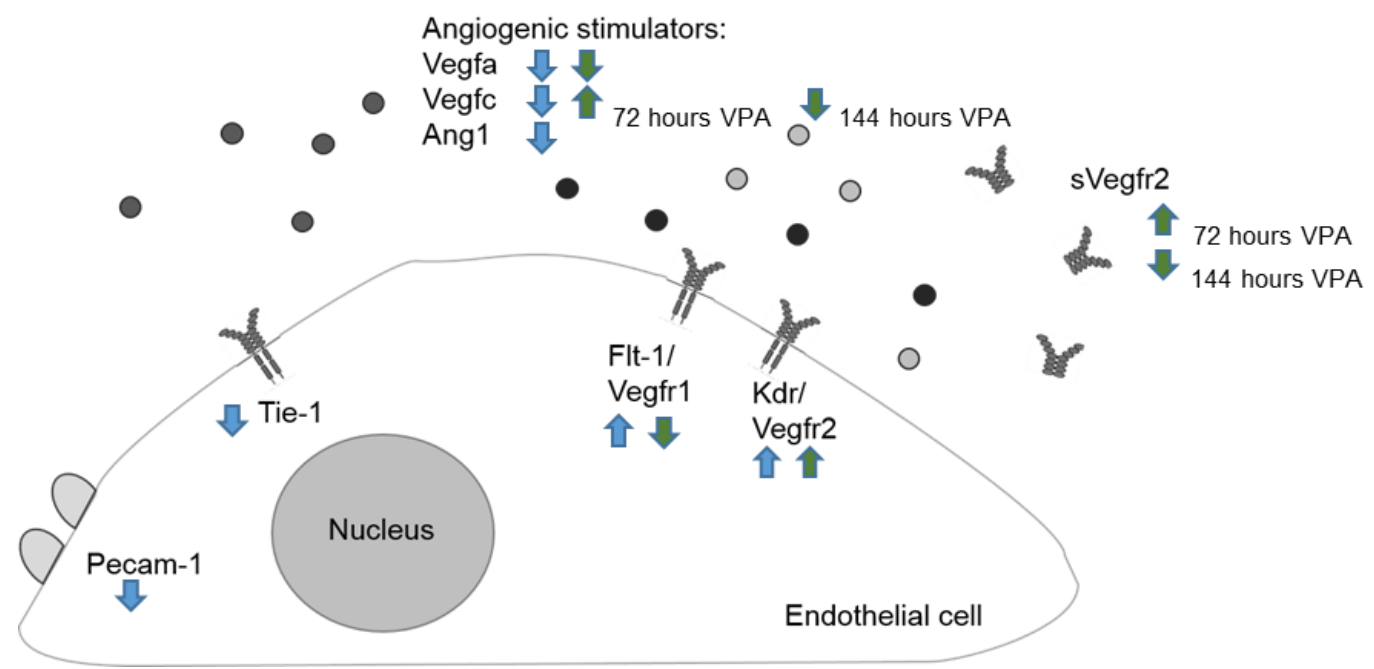

Fig. 4.5: Expression of angiogenesis markers in 2E and PC-3 cells after in vitro VPA treatment. The expression levels of several angiogenesis markers were analyzed in 2E cells (blue arrows) and PC-3 cells (green arrows) after VPA treatment and compared to untreated controls. Black, dark and light grey circles represent the angiogenic stimulators Vegfa, Vegfc and Ang1.

Taken together, in 2E and PC-3 PCa cells not only blood- but also lymphangiogenesis must be impaired after VPA treatment, since the expression of blood- and lymphangiogenic-stimulators and of their respective receptors was mainly downregulated and the lymphangiogenesis inhibitor sVEGFR2 was upregulated. However, in PC-3 cells the duration of VPA treatment seems to have an important impact on the deregulation of angiogenesis markers as in some cases (Vegfc and sVegfr2) the expression deregulation observed after 72 hours VPA treatment was contrary to the observation after 144 hours of VPA treatment. 
Nonetheless, the question remains unresolved why the expression of the receptors Flt-1/Vegfr1 and $K d r /$ Vegfr2 is upregulated in 2E cells and PC-3 after VPA treatment. The KDR/VEGFR2 signaling pathway was even shown to be activated after VPA treatment by an unknown mechanism. It is probably not activated by binding of Vegfa or Vegfc, since their expression was reduced.

The effect of in vivo VPA treatment on the expression of the angiogenesis markers was also investigated. Therefore, prostate tumor tissue and prostate tissue of VPA-treated TRAMP mice was used. In prostate tissue, of the six analyzed angiogenesis markers (Vegfa, Flt-1/Vegfr1, Kdr/Vegfr2, Ang-1, Tie-2 and Pecam-1) only Flt-1/Vegfr1 was statistically significant downregulated in VPA-treated mice as compared to controls (Fig.4.6). Ang1, Tie-1, Kdr/Vegfr2 and Pecam-1 all had a reduced expression after VPA treatment in the mouse prostate, but this effect was not statistically significant. Prostate tissue was isolated from mice at the age of 16 weeks, when TRAMP mice should have developed PIN already, and almost no statistically significant effect of VPA treatment on the expression of angiogenesis markers was observed (except Flt-1/Vegfr1 downregulation). It can be hypothesized that the expression of angiogenesis markers is not severely affected in prostates of 16-week-old TRAMP mice, because at that age these mice did not develop adenocarcinomas and tumor angiogenesis seems to play an inferior role.

In the prostate tumor tissue, the expression of Ang1, Vegfa, Tie-1 and Kdr/Vegfr2 was statistically significant reduced in VPA-treated TRAMP mice as compared to controls (Fig.4.6). Pecam-1 was also downregulated after VPA treatment, although not statistically significant, and Flt-1/VEGFR1 was similar expressed in prostate tumors of VPA-treated mice and control-treated mice.

This indicates that VPA treatment is especially effective in downregulating angiogenesis related genes in more advanced PCa and not in the very early PCa stages (e.g. PIN) when there has no adenocarcinoma developed yet. The effect of VPA on angiogenesis especially in the more advanced PCa could imply a decrease or inhibition of tumor cell proliferation. 


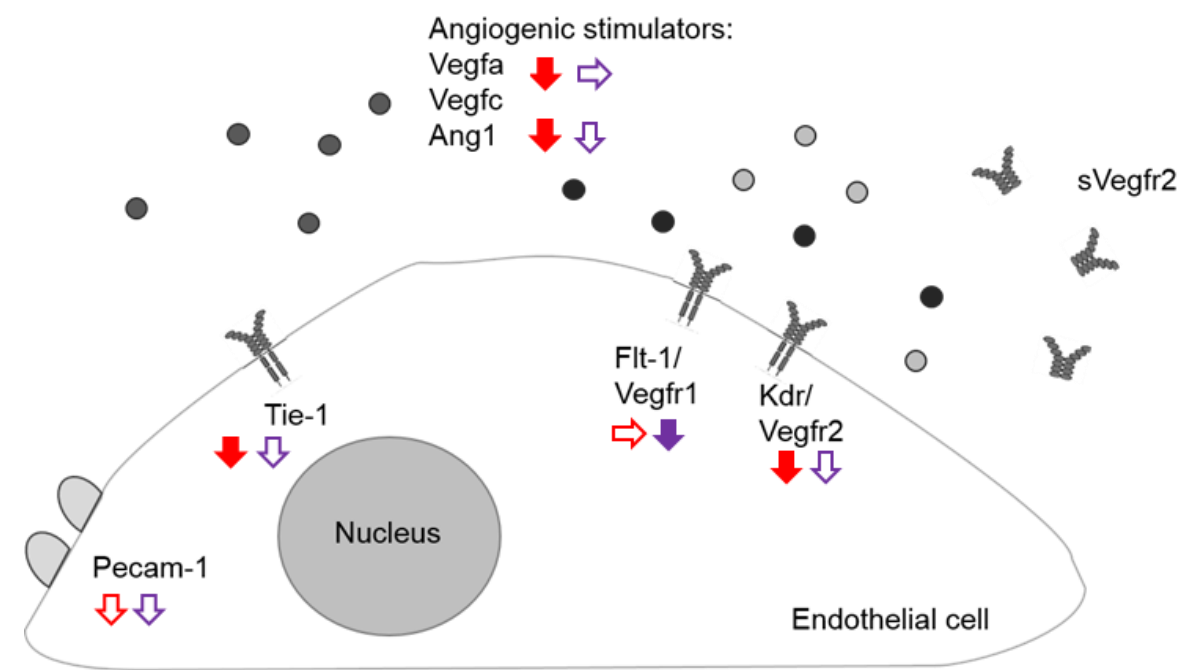

Fig. 4.6: Expression of angiogenesis markers in prostate tissue and prostate tumor tissue of in vivo VPA treated TRAMP mice. The expression of several angiogenesis markers was analyzed in prostate tumor tissue (red arrows) and prostate tissue (purple arrow) of in vivo VPA-treated TRAMP mice and compared to untreated controls. Filled arrows indicate statistically significant results, unfilled arrows indicate not statistically significant results. Black, dark and light grey circles represent the angiogenic stimulators Vegfa, Vegfc and Ang1.

Within the present study it could be shown that VPA treatment reduces the expression of several angiogenesis markers in PCa cells and in prostate tumor tissue, indicating an angiogenesis inhibition. In vitro VPA treatment reduced the expression of Vegfa, Vegfc, Ang1, Tie-1 and Pecam-1 in murine 2E PCa cells and of VEGFA and FLT-1/VEGFR1 in human PC-3 PCa cells. VEGFC and SVEGFR2 expression, dependent on the duration of VPA treatment, was increased after 72 hours and reduced after 144 hours of VPA treatment. In vivo VPA treatment exerts an angiogenesis inhibition since the expression of almost all angiogenesis markers tested was reduced as compared to controls. Especially in prostate tumor tissue this effect was statistically significant, indicating that VPA treatment is effective in downregulating angiogenesis-related genes in more advanced PCa as compared to the very early PCa stage.

Solely the overexpression of the two receptors Flt-1/Vegfr1 and Kdr/ Vegfr2 after VPA treatment in 2E cells and of KDR/VEGFR2 in PC-3 cells is contradictory to the picture of VPA as an antiangiogenic drug. This data is also contrary to what is known from the literature about the effect of VPA treatment on the expression of theses receptors in other cancer entities. A study by Dong et al. (2007) could show that VEGFR1 in human multiple myeloma KM3 cells was decreased upon VPA treatment (Dong et al., 2007). Additionally, in leukemia cell xenografts, a VPA-induced VEGFR2 downregulation has been shown (Zhang et al., 2014). As already mentioned above, the question why Flt-1/Vegfr1 and Kdr/Vegfr2 as well as KDR/VEGFR2 expression are 
upregulated and activated (as seen by increased phosphorylation) in PCa cells after VPA treatment remains unsolved.

To evaluate the influence of VPA on angiogenesis in more detail the chicken chorioallantoic membrane (CAM) assay, being one of the most famous angiogenesis assays, was applied in cooperation with Prof. Dr. Wilting from the Center of Anatomy, University Medical Center Göttingen. Here, both mouse and human PCa cells 2E and PC-3 were pre-treated for different time points with VPA (either no pre-treatment, pre-treatment for 24 hours or for one week) and then applied to the CAM, where they were forming tumors within one week. The tumors were harvested and subjected to macroscopic, immunohistochemical as well as molecular analysis to investigate the influence of VPA treatment on angiogenesis in vivo (Fig. 4.7).

Generally, for each CAM experiment the number of harvested tumors was very low since many eggs had to be discarded due to either contamination or death of the embryo which made evaluation of the CAM experiments very difficult.

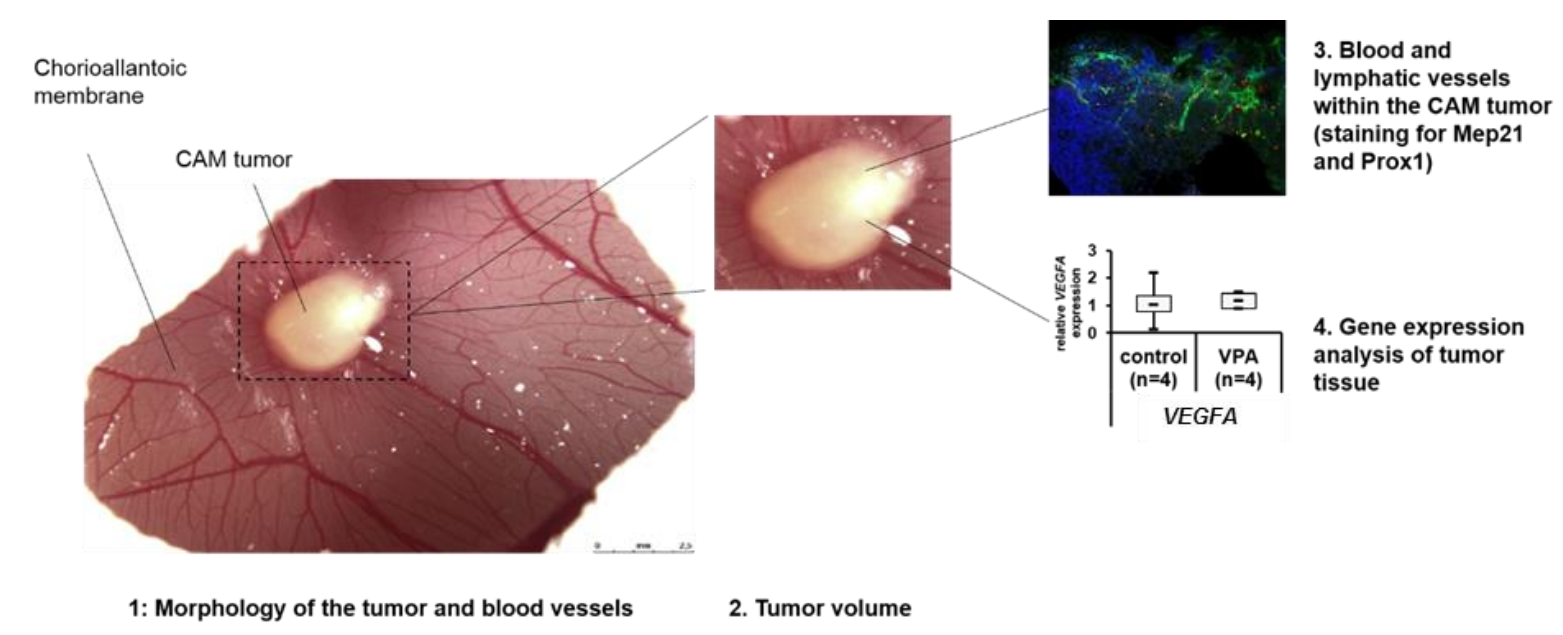

Fig. 4.7: Different approaches used to investigate the influence of VPA treatment on angiogenesis in the CAM tumor. After conducting the CAM assay four different approaches were used to investigate the influence of VPA treatment on angiogenesis. Firstly, the morphology of the CAM tumor and the surrounding blood vessels was investigated. Secondly, the tumor volume itself was calculated. Thirdly, the blood and lymphatic vessels within the CAM tumor were visualized and analyzed by immunofluorescent staining with antibodies against Mep21 and Prox1. Finally, the expression of blood and lymphangiogenesis markers in CAM tumor tissue was investigated by quantitative real-time PCR.

Macroscopic analysis revealed, that CAM tumors can be affected in three different ways, which included blood shot tumors, bloody spots within the tumor and tumors with disturbed blood vessel formation. However, when comparing the numbers of tumors affected by either one of the three phenotypes in tumors derived from VPA-treated cells with tumors grown from control cells, no general characteristic phenotype by VPA treatment could be observed. Within the experiments for one cell line the phenotypic characteristics did not correlate upon VPA treatment 
and they also did not correlate within the two cell lines. The only exception was observed for PC3 cell tumors, which in both experiments showed a reduced number of bloodshot tumors when cells were treated with VPA.

A study from Michaelis et al. (2004) in which VPA was applied to the CAM, showed reduced vessel ingrowth, the development of irregular and brittle vessels, and a markedly reduced perfusion as compared with control CAM (Michaelis et al. 2004). In the present study, in the third experiment with 2E cells and in the first PC-3 cell experiment an increase in disturbed blood vessels was detected when the cells were treated with VPA as compared to the control. However, irregular and brittle blood vessels were not characteristic for tumors of VPA-treated $\mathrm{PCa}$ cells since they were also seen in the control cells.

The CAM tumor volume was calculated by using the modified ellipsoid formula $1 / 2$ (length $x$ width$^{2}$ ). Generally, tumors grown from VPA pre-treated PCa cells had a slightly, however not significantly reduced tumor volume as compared to controls. Furthermore, tumors grown from PCa cells that were not pre-treated with VPA, only on the day of application to the CAM and three days thereafter, exhibited a slightly, but not significant increase in tumor volume compared to controls. These results indicate, that VPA pre-treatment of PCa cells might be of great importance for tumor progression in the CAM model. It is known from several studies that in vivo and in vitro VPA treatment has a significant inhibitory effect on proliferation (Shabbeer et al. 2007, Gao et al. 2007, Witt et al. 2013) which could only partially be observed in the present study. Nonetheless, this observation needs to be treated with caution since only few tumors were available for the tumor volume calculation from each experiment and each group ( $n=2-8)$. Besides, the modified ellipsoid formula was used, which is quite inaccurate since the tumor height is not considered within this formula. Also, length and width were measured from CAM tumor pictures taken on the microscope and not from the actual compound since this proved very difficult with a sliding caliper. Taking the measurements from CAM tumor cryo-sections on a slide could have been an alternative but this proved also to be difficult since some tumors slices were incomplete or deformed when transferred to the slide.

Immunofluorescence staining of CAM tumor sections with the blood vessel marker Mep21 (CD34 homolog) and with the lymphatic vessel marker Prox 1 revealed that CAM tumors derived from untreated 2E cells had very strong Mep21 staining and tumors derived from untreated PC-3 cells had very strong Prox1 and Mep21 staining. This indicates that 2E cell tumors grow mainly by blood angiogenesis, whereas PC-3 cell tumors can grow also by blood angiogenesis and to a major part by lymphangiogenesis. Upon VPA treatment of PC-3 cells, but without pre-treatment, in the distal tumor part the number of Prox1-positive cells was reduced as compared to control tumors, whereas in the proximal CAM part and in the middle part of the tumor the number of 
Pox1-positive cells was increased. This indicates that upon VPA treatment the progression of lymphatic vessels into the tumor might be inhibited.

In support of the observation mentioned above, VPA-treated PC-3 cells showed an increased expression of the lymphangiogenesis inhibitor sVEGFR2. In contrast, an increased expression of VEGFC was also observed, leading to the assumption that VEGFC competes with sVEGFR2 for the binding to VEGFR2. This could result in compensation of the VEGFC-VEGFR2 induced lymphangiogenesis, leading to either reduced lymphangiogenesis or unchanged lymphangiogenesis upon VPA treatment. And indeed, reduced lymphangiogenesis could be observed in the distal tumor part of VPA-treated CAM tumors as compared to controls.

Since untreated PC-3 CAM tumors exhibit strong Prox1 staining, indicating that growth of these tumors depends on lymphangiogenesis, it would be interesting to study if lymphangiogenesis can be inhibited, for example by use of a lymphangiogenesis inhibitor such as sVEGFR2. To date, nothing is known about the effect of VPA treatment on lymphangiogenesis, neither on cancer growth in general nor on PCa development in particular. Lymphatic vessels provide an additional route for tumor cells to metastasize, therefore, inhibiting lymphangiogenesis in PCa could be a promising approach to prevent metastatic spread and thus, making it an interesting target and possible alternative treatment option to inhibition of blood angiogenesis in cancer therapy.

Molecular evaluation of the CAM tumors exhibited some variability between the different experiments performed. Analyzing CAM tumors by quantitative real-time PCR showed that in VPA-treated PC-3 cell CAM tumors from the first CAM experiment (1 mM VPA pre-treatment for 24 hours) none of the angiogenesis markers were statistically significant deregulated as compared to controls, although their expression was slightly increased. The same result was observed for 2E cell CAM tumors from the first experiment. Solely Cp expression was statistically significant downregulated in 2E cell CAM tumors upon VPA treatment.

Increased Ccnd2 expression after VPA treatment of 2E cells was described by Witt et al. (2013) and was therefore used as positive control. Only 2E cell CAM tumors from the third experiment (3mM VPA pre-treatment for one week) exhibited a statistically significant increase in Ccnd2 expression, indicating that at least in the third experiment VPA treatment was effective, suggesting that a longer VPA pre-treatment period as well as a higher VPA concentration is therefore necessary.

Generally, the expression levels of the angiogenesis markers were rather increased in VPAtreated CAM tumors, which is contrary to results mentioned above where the expression of angiogenesis markers after in vitro and in vivo VPA treatment was rather reduced. Besides, only 
a small number of tumors were available for molecular evaluation $(n=2-5)$, which makes this study not quite representative.

Taken together, data received from the CAM experiments need to be considered with caution since the results are not quite representative due to the small number of CAM tumors. Besides, the CAM model does not seem to be the appropriate model to study VPA-induced effects on blood and lymphangiogenesis, not just because of the above mentioned aspect, but also because previous described molecular mechanism observed upon in vitro and in vivo VPA treatment could not be confirmed. Also the known functional proliferation inhibition of VPA could not be observed in VPA-treated PCa cell-derived CAM tumors.

\subsection{The role of cyclin D2 in PCa}

\subsubsection{The family of D-type cyclins}

Passage of any cell through the cell cycle is modulated by a series of proteins, of which the main players include cyclins, CDKs (cyclin-dependent kinases, positive regulators) and CDK inhibitors (negative regulators such as p21 and p27). Cyclin D2 belongs to the family of D-type cyclins alongside cyclin D1 and cyclin D3. D-type cyclins play an important role as cell cycle regulators controlling transition through G1 phase. Thereby D-type cyclins serve as regulatory co-factors for cyclin-dependent kinases (CDKs) which are inactive in the absence of a partner cyclin. Binding of the cyclin to its specific CDK activates the catalytic subunit of the CDK resulting in phosphorylation of target genes, especially of the retinoblastoma $(\mathrm{Rb})$ growth-inhibitory complex (Matsushime et al. 1992). This leads to either activation or inactivation of target proteins, which ultimately leads to the progression to the next phase of the cell cycle. Different combinations of cyclin-CDK complexes, as well as the expression of CDK-inhibitors operate during different phases of the cell cycle and determine the target genes expressed during a specific phase.

The three D-type cyclins differ not only in their structure (see Fig. 1.4), but also in their function. For example, cyclin D2 and cyclin D3 can not only bind to CDK4 and CDK6 but also to CDK2, which cyclin D1 cannot (Ewen et al. 1993). Phenotypic analyses of single cyclin D knockout mice revealed that each of the $\mathrm{D}$-type cyclins is sufficient to drive normal development of the majority of tissues (Ciemerych et al. 2002). Besides, each cyclin D exhibits distinct and mutually exclusive expression patterns in mouse embryos and some organs of adult animals. Cells risen from the epiblast and trophoblast are solely positive for cyclin D3 expression. This indicates that individual cyclin Ds may have their specific functions (Wianny et al. 1998). These specific functions may not only be CDK-dependent as seen in case of cyclin D1 which conveys cell cycle- or CDK- 
independent functions. Cyclin D1 can directly influence the expression of estrogen and androgen receptors, regulate cellular metabolism, fat cell differentiation and cellular migration (Fu et al. 2004).

In tumor cells many of the regulatory mechanism of the cell cycle can be deregulated. Especially the abnormal expression of the D-type cyclins and their CDKs is linked to cancer development and progression (Musgrove et al. 2011).

\subsubsection{Does cyclin D2 act as an oncogene or a tumor suppressor gene in PCa?}

As already mentioned above the expression of cyclin D2 is deregulated in many cancer entities. High levels of cyclin D2 messenger RNA are frequently observed in ovarian epithelial carcinomas (Milde-Langosch and Riethdorf 2003), testicular cancer (Sicinski et al. 1996), colon cancer (Mermelshtein et al. 2005) and gastric cancer (Takano et al. 2000), indicating an oncogenic role of cyclin D2 in these tumors. In glioblastoma stem cells (GSCs), cyclin D2 was also shown to play a critical role in tumorigenicity. Suppression of cyclin D2 expression by RNA interference was shown to cause G1 arrest in vitro and growth retardation of GSCs xenografted into immunocompromised mice in vivo (Koyama-Nasu et al. 2013).

However, in breast cancer (Evron et al. 2001, Fischer et al. 2002), lung cancer ((Virmani et al. 2003), pancreatic cancer (Matsubayashi et al. 2003) and some gastrointestinal tumors (Yu et al. 2003) cyclin D2 expression is frequently downregulated. Also in PCa the expression of cyclin D2 is frequently downregulated due to hypermethylation of the cyclin D2 promoter. Thereby an increased methylation status correlates with the stage of the tumor which in turn correlates inversely with the expression levels in prostatic tissue (Padar et al. 2003, Henrique et al. 2006). Increased cyclin D2 promoter methylation in PCa was also shown to correlate with faster tumor progression and poor prognosis (Padar et al. 2003, Rosenbaum et al. 2005). In contrast, cyclin D1 is frequently overexpressed in PCa and cyclin D1 has been described to act as an oncogene not only in PCa (Drobnjak et al. 2000, Ewen and Lamb 2004) but also in breast, lung, colon and hematopoietic cancer (Gillett et al. 1996, Courjal et al. 1996, Fu et al. 2004, Arnold and Papanikolaou 2005, Santarius et al. 2010, Lehn et al. 2010).

Witt et al. (2013) could show a specific re-expression of cyclin D2 after treatment with either VPA or other HDIs in PCa cells with undetectable low basal cyclin D2 expression. This phenomenon was accompanied by proliferation inhibition of PCa cells. Due to the fact that this could not be observed in fibroblast cells which exert a very high basal cyclin D2 expression, led to the hypothesis that increased cyclin D2 expression in PCa has anti-tumorigenic capacities indicating that cyclin D2 might act as a tumor suppressor (Table 4.5). 
This hypothesis was reinforced by two further studies: Kobayashi et al. (2009) stated that restoration of cyclin D2 expression in the human PCa cell line LNCaP inhibits cell proliferation (Table 4.5) and Ko et al. (2012) demonstrated that a reduced cyclin D2 expression is correlated with a poor recurrence-free survival in non-small cell lung cancer (Table 4.5). To further elucidate the role of cyclin D2 in PCa cyclin D2 downregulation studies were conducted in the present thesis, expecting that non-cancerous cells with reduced cyclin D2 expression could display features comparable to cancer cells. Therefore, NIH/3T3 fibroblast cells were used since they exhibit high basal cyclin D2 expression. Simultaneously, cyclin D2 overexpression studies were conducted with human and mouse PCa cells, expecting that these cancer cells exhibit reduced features of a cancer cell when cyclin D2 expression is increased. If cyclin D2 is indeed a tumor suppressor in $\mathrm{PCa}$, this knowledge would be of great value for possible treatment options for patients with $\mathrm{PCa}$.

Downregulating cyclin D2 expression in NIH/3T3 cells by the use of three different cyclin D2specific siRNAs resulted in an increased migration rate which was statistically significant for siRNA $B$ and siRNA $C$. The increase in the migration rate was specific after cyclin D2 downregulation, since downregulation of cyclin D1 had no effect on the migration behavior. From the literature such an effect of cyclin D2 expression on PCa cell migration was not reported. But a similar effect is known from a study with mammary epithelial cells. The authors could show that the Ets family transcription factor Pea3, which is involved in tumorigenesis especially during the metastatic process and induces migration and invasion in mammary epithelial cell models, stands in a negative feedback loop with cyclin D2. Cyclin D2 expression is lost during Pea-3induced tumorigenesis, but restoration of cyclin D2 expression resulted in decreased cell migration (Ladam et al. 2013). Here, we could show the opposing effect in fibroblast cells, i.e. reducing the cyclin D2 expression resulted in increased cell migration. Cyclin D1 is also known to influence cell migration in breast cancer cells, which is mediated through transforming growth factor beta (TGF $\beta$ ) signaling (Dai et al. 2013), but does not seem to effect cell migration in fibroblast cells.

One feature of cancer cells is their ability to grow anchorage-independent (colony forming capacity in semisolid media, Cifone and Fidler 1980). To test whether NIH/3T3 fibroblast cells with siRNA-mediated cyclin D2 downregulation possess transformation potential and can grow anchorage-independent, which normal NIH/3T3 cells would not, a soft agar assay was conducted. The capability of transformed NIH/3T3 cells, including ras-transformed NIH3T3 cells, to grow anchorage-independent has been demonstrated in many studies (Barr and Johnson 2001, Song et al. 2002, Hoque et al. 2006). The colon carcinoma cells SW620 cells, which are known to grow anchorage-independent (Coffey et al. 1986, Bullard et al. 2003) formed visible 
colonies after two weeks, whereas cyclin D2 siRNA-transfected NIH/3T3 cells exhibited no anchorage-independent growth. One possible explanation is that the transfected cells either possess no transformation potential or that the siRNA-mediated cyclin D2 downregulation is not effective over longer time periods. To exclude the latter possibility the efficiency of the cyclin D2specific siRNAs over longer time periods was tested by western blot analysis. Indeed, it could be demonstrated that the siRNA-mediated downregulation of cyclin D2 expression persists only for up to two weeks and thereafter cyclin D2 expression is restored.

Since it is known that shRNA is significantly more potent than siRNA at mediating knockdown of gene expression (McAnuff et al. 2007), a pSingle-tTs-Ccnd2-shRNA plasmid was generated which was used for transfection of NIH/3T3 fibroblast cells. The doxycycline-inducible expression of the shRNA resulted in downregulation of cyclin D2 expression which was confirmed for one clone (clone No. 11) by quantitative real-time PCR and western blot analysis. Treatment of transfected NIH/3T3 cells with $0.1 \mu \mathrm{g} / \mathrm{ml}$ doxycycline resulted in a $60 \%$ decrease in cyclin D2 expression. However, also shRNA-mediated cyclin D2 downregulation resulted in no aquired transformation potential of NIH/3T3 cells. Furthermore, cell colonies of clone No. 11 were not even growing in soft agar when simultaneously transfected with an oncogene expressing plasmid, such as pEGFP-KRAS or pEGFP-KRAS-G12V, which could support transformation potential. At this point it is important to note that the transfection efficiency for transfection with pEGFP-KRAS or pEGFP-KRAS-G12V was very poor, since only few of the transfected cells showed green fluorescence.

Additionally, the proliferation rate of doxycycline-treated NIH/3T3 clone No. 11 cells with downregulated cyclin D2 expression was comparable to control cells, although it was expected to be increased in case cyclin D2 possesses anti-proliferative functions.

It could be possible that the shRNA-mediated cyclin D2 downregulation would lead to an increased proliferation rate of $\mathrm{NIH} / 3 \mathrm{~T} 3$ cells, but that doxycycline-treatment abolishes this effect. Doxycycline-treatment is known to reduce or inhibit cell proliferation (Fife et al. 1997, Chang et al. 2010, Ahler et al. 2013). The usage of a cyclin D2-specific inhibitor would be helpful to circumvent this problem, but to date there are no cyclin D2-specific inhibitors available, only inhibitors that also inhibit other D-type cyclins, which is not intended in the present studies.

Taken together, the increased migration rate of NIH/3T3 cells transfected with a cyclin D2specific siRNA suggests that cyclin D2 possesses anti-tumorigenic capacities (Table 4.5) which could be neither confirmed nor proved invalid by both soft agar and proliferation assays. To better evaluate the functionalities of reduced cyclin D2 expression for PCa in general the studies should be repeated with a prostate epithelial cell line, for example with ATCC or PNT2 cells. But 
first it needs to be evaluated whether either one of these two cell lines encompasses high basal cyclin D2 expression, making this cell model suitable for cyclin D2 downregulation studies.

To investigate whether overexpression of cyclin D2 reduces tumorigenic features in PCa cells, cell clones and colonies were generated with stable or inducible cyclin D2 expression. Therefore, two different cyclin D2 expression vectors were generated, namely pIRES2-EGFP-CCND2 for constitutive cyclin D2 overexpression and pEBTetD-CCND2 for doxycycline-inducible cyclin D2 overexpression in PCa cells. PCa cell lines were stably transfected with both constructs and suitable cell clones were established and verified for cyclin D2 overexpression.

Studies on the functional effects of transient, stable and inducible cyclin D2 overexpression resulted in similar or increased proliferation rates compared to controls, opposing to what was expected. In the three LNCaP cells clones with stable overexpression of cyclin D2 a slightly increased proliferation was observed during the first 72 hours, whereas only after 92 hours the proliferation rate was slightly reduced for two of these LNCaP clones as compared to the control. In contrast, Kobayashi et al. (2009) could show that forced cyclin D2 expression in LNCaP cells suppressed the proliferative ability and induced cell death in LNCaP cells in a cdk-independent manner. When the forced cyclin D2 expression in LNCaP cells was knocked down again, this resulted in proliferation restoration (Kobayashi et al. 2009). A connection between increased cyclin D2 expression and proliferation inhibition has already been described in 1998 for primary and established fibroblast cells (Meyyappan et al. 1998). Inhibition of cell proliferation by either contact-inhibition or serum-deprivation was accompanied with increased cyclin D2 expression and G1 arrest. Additionally, Meyyappan and coworkers could show that passage number of the cells correlated with increased cyclin D2 expression and decreased proliferation (Meyyappan et al. 1998). In the present study, PC-3 cells with stable cyclin D2 overexpression had a not significantly increased proliferation rate. Furthermore, LNCaP colonies with doxycyclineinducible overexpression of cyclin D2 showed a significantly increased proliferation rate as compared to control cells. In the latter experiment, the concentration of doxycycline was kept as low as possible since it is known that doxycycline treatment exhibits off target effects as observed for example by a reduction or inhibition of cell proliferation (Fife et al. 1997, Chang et al. 2010, Ahler et al. 2013). Nonetheless, the proliferation rate of LNCaP colonies with inducible overexpression of cyclin D2 was increased.

In contrast to the expectation, the migration capability of LNCaP and PC-3 clones with stable cyclin D2 overexpression was also increased as compared to control cells.

Taken together, the studies conducted with cyclin D2 overexpressing cells are opposing to the expectation that cyclin D2 encompasses anti-tumorigenic capacities in PCa cells. They rather suggest that cyclin D2 exerts oncogenic potential (Table 4.5). This hypothesis is supported by 
two studies using micro RNAs (miRNAs) which target cyclin D2. Both studies could show that miRNAs (miRNA-154 and miRNA-let7a) downregulating cyclin D2 expression in human PCa cells inhibited PCa cell proliferation (Table 4.5, Dong et al. 2010, Zhu et al. 2014).

Studies conducted during this thesis on the functional consequences of cyclin D2 downregulation in mouse and human PCa cells revealed that their proliferation rate was reduced compared to luciferase control transfected cells. Upon VPA-treatment of these cells, their proliferation rate decreased further as compared to untreated control cells. However, it was expected that cells transfected with cyclin D2-specific siRNA would have an increased or comparable proliferation rate to controls after VPA treatment. The siRNA is thought to suppress VPA-induced re-expression of cyclin D2 which was shown to be accompanied with proliferation inhibition (Witt et al. 2013). Therefore, cell proliferation should not be inhibited but rather increased if cyclin D2 is directly responsible for the decreased proliferation rate observed in $2 \mathrm{E}$ cells. However, as already observed by Witt (2012), the cyclin D2-specific siRNA-mediated downregulation was not sufficient to suppress the VPA-induced re-expression of cyclin D2, as revealed by quantitative real-time PCR (data not shown). Therefore, a direct connection between VPA-induced re-expression of cyclin D2 and proliferation inhibition of PCa cells could not be demonstrated.

Taken together, from the cyclin D2 downregulation and overexpression studies in both PCa and $\mathrm{NIH} / 3 \mathrm{~T} 3$ cells conducted during the present project and due to the above mentioned limitations during the soft agar assay it could not be determined whether cyclin D2 acts as a tumor suppressor or as an oncogene in PCa. There are advantages and disadvantages for both hypotheses and further studies need to be performed to finally identify the role of cyclin D2 in PCa (Table 4.5). It would be of great interest to repeat the soft agar experiment with newly generated oncogene plasmids to find out whether cells with cyclin D2 downregulation acquire transformation potential when co-transfected with an oncogene. Finally, a combined PCa mouse model (TRAMP) with a conditional cyclin D2 knockout in the prostate $\left(\mathrm{Ccnd} 2^{\mathrm{fl} / \mathrm{fl} / \mathrm{Cre}} / \mathrm{T}^{+}\right)$would unveil with certainty its role in PCa development and progression (summarized in Table 4.5). This conditional cyclin D2 knockout mouse model $\left(\mathrm{Ccnd}^{\mathrm{fl} / f \mid} / \mathrm{Cre}^{+} / T^{+}\right)$has already been established during the present project, but at the moment no conclusions can be drawn from this mouse model, because at 18 weeks of age the $C c n d 2^{f / f f l} / \mathrm{Cre}^{+} / T^{+}$offspring is still too young to have developed a PCa (see Fig. 4.1).

Table 4.5: Comparison of the facts that cyclin D2 might act as a tumor suppressor in PCa with facts that cyclin D2 might act as an oncogene in PCa. Several studies and also results from the present project suggest that cyclin D2 might act as a tumor suppressor in PCa, whereas there are also indications that cyclin D2 might act as an oncogene in PCa. To date, there is no clear fact that proves that cyclin D2 is either an oncogene or a tumor suppressor 
and its role in PCa still remains unsolved. Two important questions that would elucidate the role of cyclin D2 in PCa could not be answered during the present project. On the one hand, the transformation potential of NIH/3T3 cells with reduced cyclin D2 expression and co-transfection with an oncogene could not be assessed by soft agar assay since transfection efficiency was very poor. On the other hand, the influence of lacking cyclin D2 expression in a conditional cyclin D2 knockout mouse model $\left(C \mathrm{Cnd} 2^{\text {fl/fl/}} / \mathrm{Cre}^{+} / T^{+}\right)$on tumor development and progression could not yet be determined since the offspring is too young to have developed a PCa.

\begin{tabular}{|c|c|}
\hline Is cyclin D2 a tumor suppressor in $\mathrm{PCa}$ ? & Is cyclin D2 an oncogene in PCa? \\
\hline $\begin{array}{l}\text { - Re-expression in PCa cells is associated with } \\
\text { proliferation inhibition (Witt et al. 2013, Kobayashi } \\
\text { et al. 2009) } \\
\text { - Reduced expression in NIH/3T3 cells results in } \\
\text { increased proliferation (Witt et al. 2013) } \\
\text { - Reduced expression in NIH/3T3 cells results in } \\
\text { increased migration rate (present study) } \\
\text { in non-small cell lung cancer, a reduced cyclin D2 } \\
\text { expression is correlated with a poor recurrence- } \\
\text { free survival (Ko et al. 2012) }\end{array}$ & $\begin{array}{l}\text { - Increased proliferation rate upon } \\
\text { overexpression in PCa cells (present study) } \\
\text { - Increased migration rate upon overexpression } \\
\text { in PCa cells (present study) } \\
\text { - miRNAs targeting cyclin D2 in human PCa cells } \\
\text { inhibited PCa cell proliferation (Dong et al. } \\
\text { 2010, Zhu et al. 2014) }\end{array}$ \\
\hline $\begin{array}{r}\text { Transformation potential of NIH/3T3 cells with } \\
\text { an oncogene? } \\
\text { - Earlier tumor development and more aggres } \\
\text { knockout? ( }\end{array}$ & $\begin{array}{l}\text { luced cyclin D2 expression and co-transfected with } \\
\text { eds to be repeated) } \\
\text { tumor in TRAMP mice with conditional cyclin D2 } \\
\text { oing experiment) }\end{array}$ \\
\hline
\end{tabular}

Besides, it might be possible that upregulation of cyclin D2 alone cannot exert the VPA-induced effects described previously, only in combination with several other factors. Hypermethylation of genes is a general effect observed in cancer, therefore not only cyclin D2 should be hyperacetylated upon VPA treatment (Witt et al. 2013), but also several other genes which together with cyclin D2 might exert the anti-tumorigenic effects (Fig.4.8).

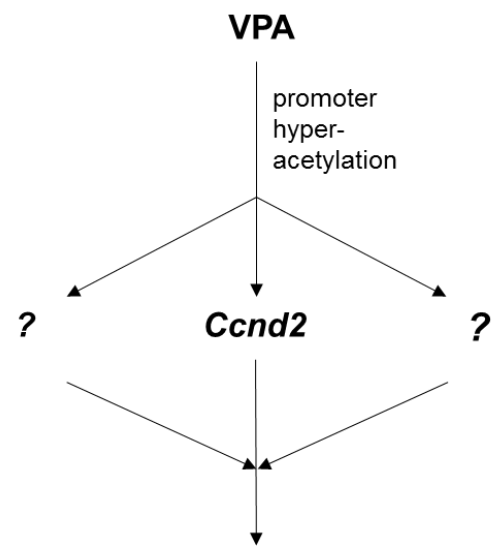

anti-tumorigenic effects (e.g. proliferation inhibition) 
Fig. 4.8: Schematic presentation how VPA treatment might exert its anti-tumorigenic effects in PCa. The VPA mediated anti-tumorigenic effects in PCa might be exerted by combination of promoter hyperacetylation of several genes, of which only Ccnd2 has been identified (Witt et al. 2013).

\subsubsection{The physiological role of cyclin D2}

To elucidate the physiological role of cyclin D2 in the mouse prostate a conditional knockout mouse model with a prostate-specific cyclin D2 deletion was established during the present project. To investigate especially the effect of cyclin D2 deletion on PCa development and progression the cyclin D2 knockout mice were crossed with TRAMP mice.

In the $\mathrm{Apc}(\mathrm{Min} /+)$ mice, a popular animal model for studies of human colon cancer (Leclerc et al. 2004), it was shown that a knockout of cyclin D2 dramatically reduced tumor growth and development (Cole et al. 2010). Cyclin D2 is known to be overexpressed in $53 \%$ of colon cancers (Mermelshtein et al. 2005), whereas it is inactivated in PCa patients due to promoter hypermethylation (Padar et al. 2003). It was shown that restoration of the cyclin D2 expression in LNCaP PCa cells resulted in reduced proliferation. Witt et al. (2013) could also show that reexpression of cyclin D2 in human and mouse PCa cells, induced by VPA treatment was associated with decreased proliferation rates, whereas fibroblast cells, in which cyclin D2 expression was not further increased by VPA treatment, showed no such proliferation inhibition, except for the L-cells. Moreover, in non-small cell lung cancer, reduced cyclin D2 expression is correlated with a poor recurrence-free survival (Ko et al. 2012). These data indicate that at least in some cancers, including PCa, increased or restored cyclin D2 expression is associated with anti-tumorigenic effects. To examine whether this holds true in vivo, in the present study a prostate-specific cyclin D2 knockout mouse model was generated, because to date such a mouse model has not been established. These conditional knockout mice were further crossed with TRAMP mice to induce PCa formation and to study the consequences of a cyclin D2 knockout on tumor development and tumor progression. If cyclin D2 indeed exerts a tumor suppressive function in PCa then TRAMP mice lacking cyclin D2 expression in the prostate epithelium should develop PCa at an earlier age or exhibit a more aggressive PCa.

Multiple conventional knockout mouse models already exist with either a single D-type cyclin knockout, double D-type cyclin knockout or triple D-type cyclin knockout (cyclin D-null mouse). Studies on single D-type cyclin knockout mice showed that they were all viable but exhibited different phenotypes. In cyclin D1 knockout mice the proliferation of breast epithelium was impaired during pregnancy whereas in female cyclin D2 knockout mice the proliferation of ovarian granulosa cells in response to follicle-stimulating hormone $(\mathrm{FSH})$ was inhibited, resulting 
in infertility. Knockout of cyclin D2 resulted in cerebellar abnormalities and impaired neurogenesis (Huard et al. 1999, Kowalczyk et al. 2004). Cyclin D3 knockout mice exhibited disturbed development of immature T-lymphocytes. The predisposition for different cancer types was also divert in the single D-type cyclin knockout mice. A knockout of cyclin D1 expression led to resistance of developing breast cancer promoted by Ras and Neu oncogenes. Cyclin D2 knockout mice had a decreased susceptibility for cancer of the gonads, whereas cyclin D3 knockout mice had a decreased susceptibility for skin papilloma and Myc-promoted oral mucosa tumors (summarized from Kozar and Sicinski 2005).

Studies on mice that express only a single D-type cyclin showed that cyclin D1-only mice developed severe megaloblastic anemia, cyclin D2-only mice presented neurological abnormalities, and cyclin D3-only mice lacked normal cerebella (Ciemerych et al. 2002). These studies indicate that the D-type cyclins possess some individual functions, but also general functions that can be compensated by the other D-type cyclins. Even the lack of all three D-type cyclins in cyclin D-null mice (cyclin D1 $\left(^{(-)}\right) \mathrm{D} 2\left(^{-{ }^{-}}\right) \mathrm{D} 3\left(^{-{ }^{-}}\right)$showed that cyclins are dispensable for cell cycle progression. These mice developed phenotypically and histopathologically comparable to control mice until embryonic day 13.5 (E13.5, Kozar et al. 2004) when most of the organs are already completely developed. At E17.5 cyclin D1 $\left(^{-/}\right) \mathrm{D} 2\left(^{(-)}\right) \mathrm{D} 3\left(^{-{ }^{-}}\right)$mice died due to heart abnormalities combined with a severe anemia. Furthermore, this study showed that Dcyclins are critically required for the expansion of hematopoietic stem cells, whereas cyclin Ddeficient fibroblasts proliferate nearly normally. Additionally, mouse embryonic fibroblast (MEF) cells lacking D-cyclins displayed reduced susceptibility to oncogenic transformation (Kozar et al. 2004).

For the present study, Ccnd2 $2^{f / f t}$ mice and $P B-C r e 4^{+}$mice with a C57/Bl6 background were purchased and their colonies established by mating with C57/BI6 wild type mice. PB-Cre $4^{+}$mice contain a second-generation composite probasin promoter, the ARR2PB promoter, which was modified to contain two androgen response elements (ARR) in the PB promoter (Zhang et al. 2000). This 0.5-kb PB promoter fragment maintains reliable prostate-specificity and simultaneously provides very high transgene expression in transgenic mice (Zhang et al. 2000). Mating of $C c n d 2^{f / / f l}$ mice with $\mathrm{PB}-\mathrm{Cre}^{+}$mice resulted in homozygous $\mathrm{Ccnd} 2^{\mathrm{fl} / f /} / \mathrm{Cre}^{+}$mice as verified by genotyping PCR. The prostate-specific knockout of cyclin D2 could be partially confirmed by PCR analysis of different mouse tissues of homozygous Ccnd2 $2^{f / f t /} / \mathrm{Cre}^{+}$. Only in the prostate tissue the band for the deleted Ccnd2 allele could be detected, however, also a specific band for the floxed Ccnd2 allele was observed. Quantitative real-time PCR and western blot analyses of different tissues revealed that cyclin D2 expression in the prostate was not reduced, 
which is contrary to the expectation from a homozygous, prostate-specific cyclin D2 knockout mouse.

One possible explanation for this observation could be that not a pure population of epithelial cells of the prostate was used for genotyping PCR, quantitative real-time PCR and western blot analyses. The probasin promoter in the knockout construct ensures specific expression of the Cre recombinase exclusively in prostate epithelial cells, therefore only these cells should exhibit a cyclin D2 knockout. For genotyping PCR, quantitative real-time PCR and western blot analyses the whole prostate gland was used, which consists of two generic cell types, i.e. epithelial cells and stromal cells. Epithelial cells form glands that are composed of the luminal secretory and basal cell types and rare neuroendocrine cells. The stroma surrounding the prostatic glands contains smooth muscle cells and fibroblasts (Cunha et al. 1996). Blood vessels, peripheral nerves and ganglia, and tissue-infiltrating white blood cells are additional constituent cell elements of the normal adult human prostate. The fact that the prostate is a solid organ makes the isolation of a specific cell type for a knockout verification problematic (Liu and True 2002).

Ongoing experiments to verify the prostate-specific knockout of cyclin D2 include immunohistochemistry, but to date no suitable antibody for mouse cyclin D2 has been identified, and RNA in situ hybridization (RNA-ISH). The cyclin D2-specific RNA probes were generated during the present study, but still need to undergo digoxigenin (DIG) labeling for the actual ISH procedure. If neither one of these two approaches should be successful in demonstrating the prostate-specific cyclin D2 downregulation, then microdissection should be conducted as a last alternative approach. Using microdissection, a single cell type, in this case prostate epithelial cells, can be specifically isolated from tissues consisting of multiple cell types. The isolated pure prostate epithelial cells could then be used for repetition of PCR, quantitative real-time PCR and western blot analyses.

The putative conditional cyclin D2 knockout mice were further mated with TRAMP mice $\left(T^{+}\right)$to study the effects of prostate-specific loss of cyclin D2 expression in a PCa mouse model. Thereby, especially the effect on tumor development and progression ought to be studied. If cyclin D2 proves to be a tumor suppressor in $\mathrm{PCa}$, then it is expected that mice lacking cyclin D2 expression in the prostate epithelium would develop PCa earlier as compared to normal TRAMP mice or exhibit a more aggressive PCa. The likelihood to receive homozygous $\mathrm{Ccnd} 2^{f / f / f} / \mathrm{Cre}^{+} / T^{+}$mice when mating $\mathrm{Ccnd} 2^{f / /+} / \mathrm{Cre}^{+} / T^{+}$mice with $\mathrm{Ccnd} 2^{f / f t}$ mice is only $12.5 \%$. To date, there are seven putative homozygous $\mathrm{Ccnd}^{f / f t /} / \mathrm{Cre}^{+} / T^{+}$mice of which none has developed a palpable PCa. The oldest of these mice is 18 weeks old, therefore, this mouse is too young to have developed an adenocarcinoma yet, as compared to single-transgenic TRAMP mice which develop an adenocarcinoma at app. 28 weeks of age. Furthermore, one 16-week-old 
heterozygous Ccnd2 $2^{f /+} / \mathrm{Cre}^{+} / T^{+}$mice had already developed a palpable PCa and had to be sacrificed. Based on the age of this heterozygous mouse it should not have developed a PCa but rather a PIN according to the tumorigenic time course in the TRAMP mouse model. Development of $\mathrm{PCa}$ in heterozygous double transgenic Ccnd2 ${ }^{f /+} / \mathrm{Cre}^{+} / T^{+}$mice at such a young age could suggest that even the loss of one cyclin D2 allele in the prostate of TRAMP mice could be sufficient to induce a more severe PCa as compared to single transgenic TRAMP mice. Nonetheless, at the present state of the experiment no definite conclusion can be drawn on the physiological role of cyclin D2 in PCa development and progression in vivo (Table 4.5, section 4.4.2).

The fact that not all TRAMP mice in our institute developed PCa and that these mice often develop seminal vesicle carcinoma, which very rarely is accompanied with development of PCa, as observed in our institute and also known from the literature (Tani et al. 2005, Yeh et al. 2009), led to the search for a more suitable PCa mouse model. In the literature there are other disadvantages of the TRAMP mouse model stated, for example that the carcinoma developed from TRAMP mice are of neuroendocrine origin (Chiaverotti et al. 2008) and not of epithelial origin as compared to the majority of human PCa cases (Abrahamsson 1999). Also, the PCa in TRAMP mice rarely metastasizes to the bone, as it is the case in humans, but rather to lymph nodes and lung (Gingrich et al. 1997). Another limiting factor for the use of TRAMP mice in PCa studies is the inherent use of the probasin promoter which is regulated by androgens (Matuo et al. 1989, Kasper et al. 1998). When it comes to interpreting consequences of castration this will be confounded by the possibility that observed androgen sensitivity is due to downregulation of transgene expression. Furthermore, as already mentioned, use of the non-physiological SV40 T antigen leads to prostate epithelium-specific inactivation of $p R b$ and $p 53$, the major targets of SV40 T antigen, resulting in formation of neuroendocrine tumors with metastasis potential to distant organs. The same is observed by prostate-specific inactivation of $p R B$ and $p 53$ (Zhou et al. 2006). Consequently, the phenotype of TRAMP mice may reflect the consequences of RB and p53 pathway inactivation. Besides, the relatively short kinetics of PCa development differ from the characteristically slow development of PCa in humans (Gingrich et al. 1999).

The PCa mouse model in which the tumor suppressor gene phosphatase and tensin homolog deleted from chromosome 10 (Pten, Song et al. 2012) is excised seems to be a suitable alternative to the TRAMP mouse model. It is the most frequently used genetically-engineered mouse model, although very few human PCa patients have loss of both PTEN alleles. Approximately $23 \%$ of human high grade PIN, $69 \%$ of localized PCa (Yoshimoto et al. 2006) and $86 \%$ of metastatic castration resistant PCa (Holcomb et al. 2009) exhibit PTEN deletions. PTEN is a phosphatase which removes a phosphate group from phosphatidylinositol 3,4,5- 
triphosphate $\left(\mathrm{PIP}_{3,4,5,)}\right.$ resulting in downregulation of the Akt/m-Tor signaling pathway, leading to decreased cell proliferation and survival (Cantley and Neel 1999). PTEN is of considerable importance for $\mathrm{PCa}$ because of its relevance for regulating androgen receptor signaling (AbateShen and Shen 2000, Shen and Abate-Shen 2007, 2010) and its loss has been linked to many cancers, including PCa (Cairns et al. 1997). Due to the fact that Pten conditional knockout mice resemble the principal driving event of human $\mathrm{PCa}$ in $\mathrm{PCa}$ of mice and also represents the human course of the disease from PIN to metastatic, castration resistant PCa of epithelial origin (Wang et al. 2003, Trotman et al. 2003) it was chosen for mating with Ccnd2//f/l Cre ${ }^{+}$mice instead of TRAMP mice. One other major benefit in using Pten conditional knockout mice instead of TRAMP mice for the generation of conditional cyclin D2 knockout mice is that there will be no further interference with other cell cycle- associated factors, solely the cyclin D2 knockout. PTEN is known to induce cell cycle arrest by negatively regulating especially cyclin D1, but also the other D-type cyclins (Radu et al. 2003, Diao and Chen 2007). PTEN was also shown to downregulate cyclin D2 expression (Huang et al. 2007). Therefore, loss of PTEN in conditional cyclin D2 knockout mice should have no further negative effect on cell cycle regulators. Using TRAMP mice, the SV40 $\mathrm{T}$ antigen would inactivate $p R b$ and $p 53$, two major cell cycle components, which would distort the outcome of a conditional cyclin D2 knockout. Thus, the phenotype of TRAMP mice with a conditional cyclin D2 knockout may also reflect the consequences of RB and p53 pathway inactivation. The idea is to solely interfere with cyclin D2 and no other cell cycle components since the physiological role of cyclin D2 ought to be investigated. The Pten conditional knockout mice fulfills this criteria and was therefore purchased and the line just recently established. Due to the advanced stage of this thesis and the accompanied time limitation it was not possible to set up the mating of Pten conditional knockout mice with prostate-specific cyclin D2 knockout mice during this project. Once the physiological function of cyclin D2 in PCa is fully understood, this knowledge can be transferred to reveal its pathologic function and might help in the development of new therapeutic strategies for the treatment of PCa.

\subsection{Perspectives}

\subsubsection{The histone deacetylase inhibitor VPA in PCa}

The influence of in vitro and in vivo VPA treatment on PCa has been investigated by several research groups. In the present study, previously described molecular mechanism in mouse PCa 2E cells induced by VPA treatment could be partially confirmed in prostate and prostate tumor 
tissue of in vivo VPA-treated TRAMP mice. The expression of one candidate gene, namely cyclin D2, was also investigated in murine prostate tumor tissue by immunohistochemistry, but the used antibody was not specific. It would be important to verify the deregulation of the candidate genes upon VPA treatment in the prostate tumor tissue samples, therefore, new cyclin D2 antibodies should be obtained for immunohistochemical evaluation of the prostate tumor tissue. During the present study it has been shown that VPA exerts anti-angiogenic effects in the mouse PCa 2E cells and the human PCa cells PC-3, but also in prostate tumor tissue of VPA-treated TRAMP mice, mainly by deregulation of angiogenesis markers. This emphasizes the need for further studies on VPA as a putative anti-angiogenic agent in PCa, since to date very little is known about the anti-angiogenic effects of VPA in cancer. At present, especially the influence of VPA on lymphangiogenesis and tumor growth has not been described in the literature, thus, opening a new field in cancer research since formation of new lymphatic vessels provides an additional route for tumor cells to metastasize. In the present study, VPA treatment has been shown to influence the expression of blood- and lymphangiogenesis-related genes in mouse and human PCa cells, highlighting the beneficial effect of VPA as an anti-cancer drug. Here, it would be also interesting to study if VPA directly influences the acetylation status of the angiogenesisrelated genes, which could be examined by chromatin immunoprecipitation studies.

In mouse PCa 2E cells it was shown that VPA treatment reduced the expression of several angiogenesis markers, whereas the expression of the two receptor tyrosine kinases Flt-1/Vegfr1 and $K d r / V e g f r 2$ was increased after VPA treatment. In case of $K d r / V e g f r 2$ this was accompanied by increased activation of the receptor signaling pathway, as seen by enhanced phosphorylation status of the receptor. Since the expression of the two ligands able to bind to Kdr/Vegfr2, namely Vegfa and Vegfc, was downregulated upon VPA treatment the question remains open which ligand-binding induces the receptor activation. Vegfd is also known to bind to Kdr/Vegfr2, therefore its expression status upon VPA treatment should be examined as well. To identify the candidate which activates the Vegf-receptor signaling pathway would be of great interest for further studies finding an approach to reduce Vegf-ligand expression or to prevent ligand-binding which would reduce or prevent signaling of the Vegf-receptor pathway and thereby inhibit formation of blood and lymphatic vessels. Furthermore, one should also investigate the expression of Vegfr3 upon VPA treatment to exclude that lymphangiogenesis is induced by this signaling pathway.

One major interesting point to investigate would be whether in vivo VPA treatment influences angiogenesis at the protein level in prostate tumor tissue of TRAMP mice as compared to controls. Therefore, microvessel density could be calculated upon CD31 or CD34 staining of prostate tumor sections. 
By immunofluorescent staining using a Prox1-specific antibody, which is a marker for lymphatic vessels, it was shown that PC-3 cell CAM tumors derived from untreated and VPA-treated cells, exhibit major staining for Prox1, indicating PC-3 cell derived tumors grow mainly by lymphangiogenesis. Further studies using a lymphangiogenesis inhibitor, such as sVegfr2 would be very important to demonstrate whether lymphangiogenesis can be inhibited or reduced in PCa cells. Therefore CAM experiments could be conducted using PC-3 cells treated with sVEGFR2 and compared to untreated controls. If indeed a reduction in lymphatic staining is observed in the sVEGFR2 treated tumors, then further studies are necessary to investigate the potential of lymphangiogenesis-inhibitors for the treatment of $\mathrm{PCa}$, for example in mouse in vivo studies.

The Prox1 staining was quantified for CAM tumor raised from PC-3 cells that were not pretreated with VPA. Thereby a reduction in Prox1-positive cells was observed in the distal tumor part of tumors derived from VPA-treated cells as compared to controls. It would be highly interesting if different VPA concentrations or different VPA pre-treatment procedures also influence lymphatic vessel formation. Therefore the number of Prox1 positive cells should be quantified also for other CAM experiments conducted, including $2 \mathrm{E}$ cell CAM experiments.

\subsubsection{The cell cycle regulator cyclin $\mathrm{D} 2$ in $\mathrm{PCa}$}

One major aim of this study was to investigate the role of cyclin D2 in PCa. Previously, it was shown by Witt (2012) that cyclin D2 exerts a specific role in PCa and it was hypothesized to function as a putative tumor suppressor in $\mathrm{PCa}$.

Firstly, it would be interesting to identify direct interaction partners of cyclin D2, for example by a Yeast-two-Hybrid-experiment using a prostate-specific library. This could contribute to the understanding of specific functions of cyclin D2 in the prostate and how they are exerted.

In the present study, cyclin D2 was overexpressed in PCa cells and the functional effects were analyzed. It was proposed that cyclin D2 overexpressing PCa cells would exhibit less features of cancer cells if cyclin D2 acts indeed as a tumor suppressor in PCa. However, migration and proliferation rates of cyclin D2 overexpressing PCa cell were rather increased than reduced, suggesting that cyclin D2 does not function as a tumor suppressor gene in PCa cells. Cyclin D2 expression was also downregulated in fibroblast cells, since they exert high basal cyclin D2 expression in contrast to PCa cells and the functional effects were analyzed. It was proposed that NIH/3T3 cells exhibiting reduced cyclin D2 expression would change their phenotype into the direction of cancer cells in case cyclin D2 is a tumor suppressor. Indeed, NIH/3T3 cells with reduced cyclin D2 expression displayed an increased migration rate. In contrast, the proliferation 
rate of NIH/3T3 cells was unchanged and a shift in the transformation potential could also not be observed, not even after transfection with an oncogene, for which the very low transfection efficiency could be responsible. Therefore, it would be very important to generate or obtain new oncogene expression vectors and transfect NIH/3T3 cells with inducible cyclin D2 downregulation with these vectors. When transfection efficiency is optimal it should be determined by soft agar assay whether fibroblast cells with reduced cyclin D2 expression exhibit a shift in the transformation potential.

Generally, the studies in which cyclin D2 was downregulated, either by siRNA or shRNA, should be repeated with prostate epithelial cells. In the present study, fibroblast cells were used since they were available in the institute and exhibit high basal cyclin D2 expression. In order to optimize the evaluation regarding the functionalities of reduced cyclin D2 expression for PCa in general the studies should be repeated with a prostate epithelial cell line which exhibits high basal cyclin D2 expression. Human or mouse PCa cells are not suitable for these future experiments since they display undetectable low cyclin D2 expression levels.

Downregulation of cyclin D2 by transfection with a shRNA expression vector could be not sufficient in completely silencing cyclin D2, since the expression of cyclin D2 was downregulated by only $60 \%$. During this study the transcription activator-like effector nuclease (TALEN) technique was also consulted to induce cleavage of the cyclin D2 DNA sequence but it could not be applied, since molecular cloning of the specific domains was not successful. Another option to induce downregulation of cyclin D2 would be the use of a cyclin D2-specific inhibitor, but to date there are none available. At present, available cyclin inhibitors inhibit cyclin D2 as wells as other cyclin Ds simultaneously, but this phenomenon would distort the results.

Taken together, the in vitro studies do not really confirm the hypothesis that cyclin D2 has a tumor suppressive function in PCa. Therefore, the in vivo studies on tumor development and progression in TRAMP mice with a prostate-specific knockout of cyclin D2 are of major importance to clarify the role of cyclin D2 in PCa.

The most important aspect concerning the in vivo studies is the confirmation of the prostatespecific deletion of cyclin D2 in the generated transgenic mice. By PCR analysis of prostate tissue the knockout could only be partially confirmed, presumably because a mixture of prostate cells was analyzed and not pure epithelial cells in which the cyclin D2 knockout should occur. Other approaches conducted during this study to verify the conditional deletion of cyclin D2 include RNA in situ hybridization (RNA-ISH) and immunohistochemical staining of prostate sections with a cyclin D2-specific antibody. Both approaches are still ongoing and need to be continued. If neither one of these techniques is able to demonstrate the conditional knockout 
then microdissection of prostate tissue should be conducted, where only prostate epithelial cells are being excised with a laser and which can then be used for repetition of PCR analyses.

To date, the in vivo studies on tumor development and tumor progression in the conditional cyclin D2 knockout mice are still ongoing because the available mice are too young to have even developed an adenocarcinoma yet. Therefore, at the present state of the in vivo experiment no definite conclusion can be drawn on the physiological role of cyclin D2 in PCa which implies continuation of the in vivo studies. Besides, the TRAMP mouse model should be switched to the Pten knockout mouse model since it exhibits several benefits over the TRAMP mouse model described in section 4.4.3. The Pten knockout mouse model has just recently been established and can now be used for further in vivo studies.

It would also be interesting to analyze the normal prostate tissue of conditional cyclin D2 knockout mice (Ccnd2 $2^{f / f / l} / \mathrm{Cre}^{+}$and $\left.\mathrm{Ccnd} 2^{f / / f l} / \mathrm{Cre}^{+} / T^{+}\right)$histologically, for example by H\&E staining. Thereby the physiological role of cyclin D2 in normal prostate development can also be evaluated. 


\section{Summary}

In the first project of the present study, the influence of in vivo VPA treatment on the expression of previously identified candidate genes associated with PCa (Witt, 2009) was subject to investigation. Besides, VPA-mediated molecular effects on angiogenesis-related genes and on angiogenesis in vivo by use of the CAM model should be analyzed.

In the second project, the role of cyclin D2 in PCa should be further elucidated. It was hypothesized that cyclin D2 exerts tumor suppressive function in PCa (Witt, 2012) which was further investigated in the present study by functional experiments of cells overexpressing cyclin D2 and of cells with reduced cyclin D2 expression. To elucidate the physiological role of cyclin $\mathrm{D} 2$ in PCa in vivo a conditional cyclin D2 knockout mouse model was generated and crossed with TRAMP mice.

\subsection{The histone deacetylase inhibitor valproic acid in $\mathrm{PCa}$}

Studies on possible agents for PCa therapy are indispensable since treatment options for early PCa stages are associated with severe side effects and treatment options for mCRPC solely prolong the patients live and relieve pain, but they are not curative and death is inevitable.

One of the two main parts of the present study was to investigate valproic acid (VPA) as a putative candidate for PCa therapy. VPA is a histone deacetylase inhibitor implicated by many studies as a putative agent for the treatment of cancer, including PCa. Previously, it has been described that VPA treatment decreases the expression of Cxcl15, Cp1, Cp2, Rcbtb2 and Lif and increases the expression of Uchl1, Ptprn and Ccnd2 in the murine PCa cells $2 \mathrm{E}$ in a timeand concentration-dependent manner (Witt et al. 2013).

In the present study, the downregulation of $C p 2$ and Lif could be confirmed by quantitative realtime PCR in prostate tumor tissue of VPA-treated TRAMP mice. Densitometrical evaluation of a western blot confirmed the increased Ccnd2 expression upon VPA treatment observed in 2E cells also in prostate tumor tissue of VPA-treated TRAMP mice.

In prostate tissue of in vivo VPA-treated TRAMP mice the candidate gene expression pattern was more similar to the previously obtained microarray data (Witt. 2009). The downregulation of Cp2 as well as the upregulation of Ptprn and Uchl1 could be confirmed in prostate tissue of in vivo VPA-treaded TRAMP mice.

Taken together, molecular effects observed by in vitro VPA treatment of mouse PCa cells (2E) could be partially confirmed in prostate tumor tissue and to a greater extend in prostate tissue 
of in vivo VPA-treated TRAMP mice. These results demonstrate the benefits of VPA as a possible treatment opportunity for PCa especially in early PCa stages such as PIN.

In the present study, the investigation of the influence of VPA treatment especially on tumor angiogenesis as well as on lymphangiogenesis was of major interest. The expression of several angiogenesis markers, including Vegfa, Vegfc, Ang1, Flt-1/Vegfr1, Kdr/Vegfr2, Tie-1, the soluble Vegfr2 receptor (sVegfr2) and Pecam-1 was analyzed by quantitative real-time PCR after in vitro VPA-treatment of murine 2E PCa cells and in vivo VPA treatment of TRAMP mice. In murine $\mathrm{PCa} 2 \mathrm{E}$ cells, the expression of almost all angiogenesis markers mentioned above (i.e. Vegfa, Vegfc, Ang1, Tie-1 and Pecam-1) was decreased in a concentration- and partially timedependent manner upon VPA treatment. However, both Flt-1/Vegfr1 and Kdr/Vegfr2 were significantly upregulated after VPA treatment and in case of $K d r / V e g f r 2$ it was accompanied by increased activation of the receptor signaling pathway as identified by western blot analysis. In human PC-3 cells, the downregulation of angiogenesis markers could be partially confirmed but dependent on the duration of VPA treatment.

In vivo VPA treatment also reduced the expression of several angiogenesis markers, as can be seen by statistically significant reduced Flt-1/Vegfr1 expression in prostate tissue and Ang1, Vegfa, Tie-1 and Kdr/Vegfr2 expression in prostate tumor tissue. These results indicate, that VPA treatment has a greater effect on the downregulation of angiogenesis markers in more advanced PCa than in very early tumor stages.

Taken together, in vivo and in vitro VPA treatment effects both blood and lymphangiogenesis, as it could be observed by reduced expression of the angiogenic ligands and their receptors and by the upregulation of sVEGFR2, a lymphangiogenesis inhibitor, the latter describing a novel effect of VPA treatment on lymphangiogenesis.

For in vivo studies of VPA treatment regarding the influence on angiogenesis several CAM experiments were conducted with mouse $2 \mathrm{E}$ and human PC-3 PCa cells, which were treated with different concentrations of VPA and received different pre-treatments. The CAM experiments were not quite successful, because the tumor outcome was very low and macroscopic, immunohistochemical and molecular evaluation of these tumors did not yield the expected results, such as a reduced tumor volume or reduced expression of angiogenesis markers.

Taken together, in vivo and in vitro VPA treatment was shown to modulate the expression of candidate genes associated with PCa. Furthermore, it was shown to modulate not only the expression of angiogenesis-related genes (i.e. Vegfa, Ang1, Flt-1/Vegfr1, Pecam-1 and Tie-1) but also of lymphangiogenesis related genes (i.e. Vegfc, Kdr/Vegfr2 and sVegfr2) which might 
be associated with angiogenesis inhibition. These data further highlight the beneficial effect of VPA treatment for PCa therapy.

\subsection{The cell cycle regulator cyclin D2 in PCa}

Cyclin D2 is a known cell cycle regulator, which was proposed to have a special function in PCa, possibly acting as a tumor suppressor (Witt, 2012).

To elucidate the role of cyclin D2 in PCa cells, LNCaP and PC-3 cell clones with a stable overexpression of cyclin D2 and $\mathrm{LNCaP}$ colonies with a doxycycline-inducible cyclin D2 overexpression were generated.

Contrary to the expectation, functional analysis of human PCa cells with a transient, stable or inducible cyclin D2 overexpression revealed that these cells exhibited a similar or slightly increased proliferation rate as compared to controls. PC-3 and LNCaP cells with stable cyclin D2 overexpression had an increased migration rate as compared to controls. These data rather indicate that cyclin D2 is not a tumor suppressor in PCa.

Functional studies of NIH/3T3 cells transfected with cyclin D2-specific siRNAs showed that these cells had an increased migration rate as compared to luciferase control transfected cells, indicating that cyclin D2 might have anti-tumorigenic capacities, nonetheless.

$\mathrm{NIH} / 3 \mathrm{~T} 3$ cells transfected with cyclin D2-specific siRNA acquired no shift in their transformation potential, which was investigated by soft agar assay, presumably because siRNA transfection is not effective over longer time periods. Therefore, NIH/3T3 clones with doxycycline-inducible cyclin D2 downregulation were generated. Their proliferation behavior was similar to that of control cells which were not treated with doxycycline. Again it was tested by soft agar assay if these cells acquired transformation potential, but this was not the case, not even when the cells were double transfected with the oncogene KRAS. For this phenomenon the insufficient transfection efficiency could be responsible.

The functional consequence of cyclin D2 downregulation in VPA-treated 2E, LNCaP, PC-3 and DU145 cells was a reduced proliferation rate, indicating that cyclin D2 is not directly responsible for the known VPA-induced proliferation inhibition. But, since it was shown that the siRNA is not sufficient to prevent the VPA-induced re-expression of cyclin D2, this result needs to be considered with caution.

The physiological role of cyclin D2 in the organism was investigated by the generation of a prostate-specific cyclin D2 knockout mouse model, the Ccnd $2^{f / f t /} / \mathrm{Cre}^{+}$mice. Ccnd $2^{f / f t /} / \mathrm{Cre}^{+}$mice were further bred to $\mathrm{TRAMP}^{+}$mice to study the influence of the prostate-specific deletion of cyclin D2 on prostate tumor development and progression. To date, there are seven 


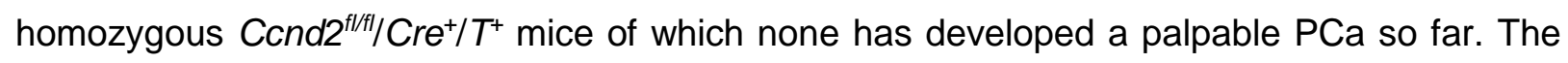

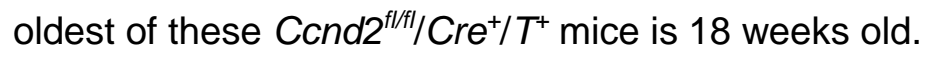

Taken together, the hypothesis that cyclin D2 could act as a tumor suppressor in PCa remains unsolved. Functional studies of cells overexpressing cyclin D2 rather point to the opposite direction, whereas functional studies of cells in which cyclin D2 is downregulated indicate that it might have anti-tumorigenic capacities. Nonetheless, the downregulation studies exhibited several limitations and should be repeated with an epithelial prostate cell line in order to make a closing statement. Besides, from the in vivo studies, which could give a definite answer on the physiological role of cyclin D2 in PCa, no conclusions can be drawn at the present state of the in vivo experiment, because these studies are still ongoing. 


\section{Bibliography}

Abate-Shen, C., and M. M. Shen. 2000. Molecular genetics of prostate cancer. Genes \& Development 14(19):2410-2434.

Abrahamsson, P. A. 1999. Neuroendocrine cells in tumour growth of the prostate. EndocrineRelated Cancer 6(4):503-519.

Adair, T. H., and J.-P. Montani. 2010. Overview of Angiogenesis.

Ahler, E., W. J. Sullivan, A. Cass, D. Braas, A. G. York, S. J. Bensinger, T. G. Graeber, and H. R. Christofk. 2013. Doxycycline alters metabolism and proliferation of human cell lines. PloS One 8(5):e64561.

Albuquerque, R. J. C., T. Hayashi, W. G. Cho, M. E. Kleinman, S. Dridi, A. Takeda, J. Z. Baffi, K. Yamada, H. Kaneko, M. G. Green, J. Chappell, J. Wilting, H. A. Weich, S. Yamagami, S. Amano, N. Mizuki, J. S. Alexander, M. L. Peterson, R. A. Brekken, M. Hirashima, S. Capoor, T. Usui, B. K. Ambati, and J. Ambati. 2009. Alternatively spliced vascular endothelial growth factor receptor-2 is an essential endogenous inhibitor of lymphatic vessel growth. Nature Medicine 15(9):1023-1030.

Almodovar, C. R. de, D. Lambrechts, M. Mazzone, and P. Carmeliet. 2009. Role and Therapeutic Potential of VEGF in the Nervous System. Physiological Reviews 89(2):607-648.

Altschul, S. F., W. Gish, W. Miller, E. W. Myers, and D. J. Lipman. 1990. Basic local alignment search tool. Journal of Molecular Biology 215(3):403-410.

American Cancer Society. Global Cancer Facts \& Figures 3rd Edition. Atlanta: American Cancer Society; 2015.

Angelucci, A., P. Muzi, L. Cristiano, D. Millimaggi, A. Cimini, V. Dolo, R. Miano, C. Vicentini, M. $P$. Cerù, and M. Bologna. 2008. Neuroendocrine transdifferentiation induced by VPA is mediated by PPARgamma activation and confers resistance to antiblastic therapy in prostate carcinoma. The Prostate 68(6):588-598.

Angiogenesis Foundation, Tumor angiogenesis, http://www.dianasaville.com, 17.01.2016.

Annicotte, J.-S., I. lankova, S. Miard, V. Fritz, D. Sarruf, A. Abella, M.-L. Berthe, D. Noël, A. Pillon, F. Iborra, P. Dubus, T. Maudelonde, S. Culine, and L. Fajas. 2006. Peroxisome proliferator-activated receptor gamma regulates E-cadherin expression and inhibits growth and invasion of prostate cancer. Molecular and Cellular Biology 26(20):75617574. 
Antonarakis, E. S., and M. A. Eisenberger. 2013. Phase III trials with docetaxel-based combinations for metastatic castration-resistant prostate cancer: time to learn from past experiences. Journal of Clinical Oncology: Official Journal of the American Society of Clinical Oncology 31(14):1709-1712.

Arce, C., C. Pérez-Plasencia, A. González-Fierro, E. de la Cruz-Hernández, A. Revilla-Vázquez, A. Chávez-Blanco, C. Trejo-Becerril, E. Pérez-Cárdenas, L. Taja-Chayeb, E. Bargallo, P. Villarreal, T. Ramírez, T. Vela, M. Candelaria, M. F. Camargo, E. Robles, and A. Dueñas-González. 2006. A proof-of-principle study of epigenetic therapy added to neoadjuvant doxorubicin cyclophosphamide for locally advanced breast cancer. PloS One 1:e98.

Arnold, A., and A. Papanikolaou. 2005. Cyclin D1 in breast cancer pathogenesis. Journal of Clinical Oncology: Official Journal of the American Society of Clinical Oncology 23(18):4215-4224.

Bach, M., S. Grigat, B. Pawlik, C. Fork, O. Utermöhlen, S. Pal, D. Banczyk, A. Lazar, E. Schömig, and D. Gründemann. 2007. Fast set-up of doxycycline-inducible protein expression in human cell lines with a single plasmid based on Epstein-Barr virus replication and the simple tetracycline repressor. FEBS Journal 274(3):783-790.

Banerjee, S., M. Dowsett, A. Ashworth, and L.-A. Martin. 2007. Mechanisms of disease: angiogenesis and the management of breast cancer. Nature Clinical Practice. Oncology $4(9): 536-550$.

Barr, S. M., and E. M. Johnson. 2001. Ras-induced colony formation and anchorageindependent growth inhibited by elevated expression of Puralpha in NIH3T3 cells. Journal of Cellular Biochemistry 81(4):621-638.

Bilusic, M., and Y.-N. Wong. 2014. Anti-angiogenesis in prostate cancer: knocked down but not out. Asian Journal of Andrology 16(3):372-377.

de Bono, J. S., C. J. Logothetis, A. Molina, K. Fizazi, S. North, L. Chu, K. N. Chi, R. J. Jones, O. B. Goodman, F. Saad, J. N. Staffurth, P. Mainwaring, S. Harland, T. W. Flaig, T. E. Hutson, T. Cheng, H. Patterson, J. D. Hainsworth, C. J. Ryan, C. N. Sternberg, S. L. Ellard, A. Fléchon, M. Saleh, M. Scholz, E. Efstathiou, A. Zivi, D. Bianchini, Y. Loriot, N. Chieffo, T. Kheoh, C. M. Haqq, H. I. Scher, and COU-AA-301 Investigators. 2011. Abiraterone and increased survival in metastatic prostate cancer. The New England Journal of Medicine 364(21):1995-2005.

de Bono, J. S., S. Oudard, M. Ozguroglu, S. Hansen, J.-P. Machiels, I. Kocak, G. Gravis, I. Bodrogi, M. J. Mackenzie, L. Shen, M. Roessner, S. Gupta, A. O. Sartor, and TROPIC Investigators. 2010. Prednisone plus cabazitaxel or mitoxantrone for metastatic castration-resistant prostate cancer progressing after docetaxel treatment: a randomised open-label trial. Lancet (London, England) 376(9747):1147-1154. 
Borowicz, S., M. Van Scoyk, S. Avasarala, M. K. Karuppusamy Rathinam, J. Tauler, R. K. Bikkavilli, and R. A. Winn. 2014. The Soft Agar Colony Formation Assay. Journal of Visualized Experiments(92).

Borre, M., B. V. Offersen, B. Nerstrøm, and J. Overgaard. 1998. Microvessel density predicts survival in prostate cancer patients subjected to watchful waiting. British Journal of Cancer 78(7):940-944.

Bottaro, D. P., and L. A. Liotta. 2003. Cancer: Out of air is not out of action. Nature 423(6940):593-595.

Bradford, M. M. 1976. A rapid and sensitive method for the quantitation of microgram quantities of protein utilizing the principle of protein-dye binding. Analytical Biochemistry 72:248254.

Braiteh, F., A. O. Soriano, G. Garcia-Manero, D. Hong, M. M. Johnson, L. D. P. Silva, H. Yang, S. Alexander, J. Wolff, and R. Kurzrock. 2008. Phase I study of epigenetic modulation with 5-azacytidine and valproic acid in patients with advanced cancers. Clinical Cancer Research: An Official Journal of the American Association for Cancer Research 14(19):6296-6301.

Brinkmann, H., A. L. Dahler, C. Popa, M. M. Serewko, P. G. Parsons, B. G. Gabrielli, A. J. Burgess, and N. A. Saunders. 2001. Histone hyperacetylation induced by histone deacetylase inhibitors is not sufficient to cause growth inhibition in human dermal fibroblasts. The Journal of Biological Chemistry 276(25):22491-22499.

Bullard, K. M., H.-R. Kim, M. A. Wheeler, C. M. Wilson, C. L. Neudauer, M. A. Simpson, and J. B. McCarthy. 2003. Hyaluronan synthase-3 is upregulated in metastatic colon carcinoma cells and manipulation of expression alters matrix retention and cellular growth. International Journal of Cancer. Journal International Du Cancer 107(5):739-746.

Byler, T. K., D. Leocadio, O. Shapiro, G. Bratslavsky, C. J. Stodgell, R. W. Wood, E. M. Messing, and J. E. Reeder. 2012. Valproic acid decreases urothelial cancer cell proliferation and induces thrombospondin-1 expression. BMC Urology 12:21.

Cai T, G. Nesi, G. Tinacci, G. Giubilei, A. Gavazzi, N. Mondaini, E. Zini and R. Bartoletti. 2011. Clinical importance of lymph node density in predicting outcome of prostate cancer patients. Journal of Surgical Research 167(2):267-272.

Cairns, P., K. Okami, S. Halachmi, N. Halachmi, M. Esteller, J. G. Herman, J. Jen, W. B. Isaacs, G. S. Bova, and D. Sidransky. 1997. Frequent inactivation of PTEN/MMAC1 in primary prostate cancer. Cancer Research 57(22):4997-5000. 
Candelaria, M., D. Gallardo-Rincón, C. Arce, L. Cetina, J. L. Aguilar-Ponce, O. Arrieta, A. González-Fierro, A. Chávez-Blanco, E. de la Cruz-Hernández, M. F. Camargo, C. TrejoBecerril, E. Pérez-Cárdenas, C. Pérez-Plasencia, L. Taja-Chayeb, T. Wegman-Ostrosky, A. Revilla-Vazquez, and A. Dueñas-González. 2007. A phase II study of epigenetic therapy with hydralazine and magnesium valproate to overcome chemotherapy resistance in refractory solid tumors. Annals of oncology: official journal of the European Society for Medical Oncology / ESMO 18(9):1529-1538.

Cantley, L. C., and B. G. Neel. 1999. New insights into tumor suppression: PTEN suppresses tumor formation by restraining the phosphoinositide 3-kinase/AKT pathway. Proceedings of the National Academy of Sciences of the United States of America 96(8):4240-4245.

Cao, G., C. D. O’Brien, Z. Zhou, S. M. Sanders, J. N. Greenbaum, A. Makrigiannakis, and H. M. DeLisser. 2002. Involvement of human PECAM-1 in angiogenesis and in vitro endothelial cell migration. American Journal of Physiology. Cell Physiology 282(5):C1181-1190.

Chamberlain, M. C. 2011. Bevacizumab for the Treatment of Recurrent Glioblastoma. Clinical Medicine Insights. Oncology 5:117-129.

Chang, W. Y. C., D. Clements, and S. R. Johnson. 2010. Effect of doxycycline on proliferation, MMP production, and adhesion in LAM-related cells. American Journal of Physiology. Lung Cellular and Molecular Physiology 299(3):L393-400.

Chateauvieux, S., F. Morceau, M. Dicato, and M. Diederich. 2010. Molecular and Therapeutic Potential and Toxicity of Valproic Acid. Journal of Biomedicine and Biotechnology 2010.

Chen-Konak, L., Y. Guetta-Shubin, H. Yahav, A. Shay-Salit, M. Zilberman, O. Binah, and N. Resnick. 2003. Transcriptional and post-translation regulation of the Tie1 receptor by fluid shear stress changes in vascular endothelial cells. FASEB journal: official publication of the Federation of American Societies for Experimental Biology 17(14):2121-2123.

Chiaverotti, T., S. S. Couto, A. Donjacour, J.-H. Mao, H. Nagase, R. D. Cardiff, G. R. Cunha, and A. Balmain. 2008. Dissociation of epithelial and neuroendocrine carcinoma lineages in the transgenic adenocarcinoma of mouse prostate model of prostate cancer. The American Journal of Pathology 172(1):236-246.

Chou, Y.-W., N. K. Chaturvedi, S. Ouyang, F.-F. Lin, D. Kaushik, J. Wang, I. Kim, and M.-F. Lin. 2011. Histone deacetylase inhibitor valproic acid suppresses the growth and increases the androgen responsiveness of prostate cancer cells. Cancer Letters 311(2):177-186.

Chou, Y.-W., F.-F. Lin, S. Muniyan, F. C. Lin, C.-S. Chen, J. Wang, C.-C. Huang, and M.-F. Lin. 2015. Cellular prostatic acid phosphatase (cPAcP) serves as a useful biomarker of histone deacetylase (HDAC) inhibitors in prostate cancer cell growth suppression. Cell \& Bioscience 5. 
Chung, T. D. K., J. J. Yu, M. T. Spiotto, M. Bartkowski, and J. W. Simons. 1999. Characterization of the role of IL-6 in the progression of prostate cancer. The Prostate 38(3):199-207.

Ciemerych, M. A., A. M. Kenney, E. Sicinska, I. Kalaszczynska, R. T. Bronson, D. H. Rowitch, H. Gardner, and P. Sicinski. 2002. Development of mice expressing a single D-type cyclin. Genes \& Development 16(24):3277-3289.

Cifone, M. A., and I. J. Fidler. 1980. Correlation of patterns of anchorage-independent growth with in vivo behavior of cells from a murine fibrosarcoma. Proceedings of the National Academy of Sciences of the United States of America 77(2):1039-1043.

Cinatl, J., R. Kotchetkov, R. Blaheta, P. H. Driever, J. U. Vogel, and J. Cinatl. 2002. Induction of differentiation and suppression of malignant phenotype of human neuroblastoma $\mathrm{BE}(2)$ $C$ cells by valproic acid: enhancement by combination with interferon-alpha. International Journal of Oncology 20(1):97-106.

Clark, J. M. 1988. Novel non-templated nucleotide addition reactions catalyzed by procaryotic and eucaryotic DNA polymerases. Nucleic Acids Research 16(20):9677-9686.

Clegg, N. J., J. Wongvipat, J. D. Joseph, C. Tran, S. Ouk, A. Dilhas, Y. Chen, K. Grillot, E. D. Bischoff, L. Cai, A. Aparicio, S. Dorow, V. Arora, G. Shao, J. Qian, H. Zhao, G. Yang, C. Cao, J. Sensintaffar, T. Wasielewska, M. R. Herbert, C. Bonnefous, B. Darimont, H. I. Scher, P. Smith-Jones, M. Klang, N. D. Smith, E. De Stanchina, N. Wu, O. Ouerfelli, P. J. Rix, R. A. Heyman, M. E. Jung, C. L. Sawyers, and J. H. Hager. 2012. ARN-509: a novel antiandrogen for prostate cancer treatment. Cancer Research 72(6):1494-1503.

Coffey, R. J., G. D. Shipley, and H. L. Moses. 1986. Production of Transforming Growth Factors by Human Colon Cancer Lines. Cancer Research 46(3):1164-1169.

Cohen, M. H., J. Gootenberg, P. Keegan, and R. Pazdur. 2007a. FDA drug approval summary: bevacizumab plus FOLFOX4 as second-line treatment of colorectal cancer. The Oncologist 12(3):356-361.

Cohen, M. H., J. Gootenberg, P. Keegan, and R. Pazdur. 2007b. FDA drug approval summary: bevacizumab (Avastin) plus Carboplatin and Paclitaxel as first-line treatment of advanced/metastatic recurrent nonsquamous non-small cell lung cancer. The Oncologist 12(6):713-718.

Cole, A., K. Myant, K. Reed, R. Ridgway, D. Athineos, G. Van den Brink, V. Muncan, H. Clevers, A. Clarke, P. Sicinski, and O. Sansom. 2010. Cyclin D2-CDK4/6 is required for efficient proliferation and tumorigenesis following Apc loss. Cancer research 70(20):8149-8158.

Coleman, R. E. 2006. Clinical features of metastatic bone disease and risk of skeletal morbidity. Clinical Cancer Research: An Official Journal of the American Association for Cancer Research 12(20 Pt 2):6243s-6249s. 
Conn, G., M. L. Bayne, D. D. Soderman, P. W. Kwok, K. A. Sullivan, T. M. Palisi, D. A. Hope, and K. A. Thomas. 1990. Amino acid and cDNA sequences of a vascular endothelial cell mitogen that is homologous to platelet-derived growth factor. Proceedings of the National Academy of Sciences of the United States of America 87(7):2628-2632.

Courjal, F., G. Louason, P. Speiser, D. Katsaros, R. Zeillinger, and C. Theillet. 1996. Cyclin gene amplification and overexpression in breast and ovarian cancers: evidence for the selection of cyclin D1 in breast and cyclin E in ovarian tumors. International Journal of Cancer. Journal International Du Cancer 69(4):247-253.

Cunha, G. R., S. W. Hayward, R. Dahiya, and B. A. Foster. 1996. Smooth muscle-epithelial interactions in normal and neoplastic prostatic development. Acta Anatomica 155(1):6372.

Dai, M., A. A. Al-Odaini, N. Fils-Aimé, M. A. Villatoro, J. Guo, A. Arakelian, S. A. Rabbani, S. Ali, and J. J. Lebrun. 2013. Cyclin D1 cooperates with p21 to regulate TGF $\beta$-mediated breast cancer cell migration and tumor local invasion. Breast Cancer Research 15(3):R49.

Dameron, K. M., O. V. Volpert, M. A. Tainsky, and N. Bouck. 1994. Control of angiogenesis in fibroblasts by $\mathrm{p53}$ regulation of thrombospondin-1. Science (New York, N.Y.) 265(5178):1582-1584.

DaSilva, J. O., G. P. Amorino, E. V. Casarez, B. Pemberton, and S. J. Parsons. 2013. Neuroendocrine-derived peptides promote prostate cancer cell survival through activation of IGF-1R signaling. The Prostate 73(8):801-812.

Davis, S., T. H. Aldrich, P. F. Jones, A. Acheson, D. L. Compton, V. Jain, T. E. Ryan, J. Bruno, C. Radziejewski, P. C. Maisonpierre, and G. D. Yancopoulos. 1996. Isolation of angiopoietin-1, a ligand for the TIE2 receptor, by secretion-trap expression cloning. Cell 87(7):1161-1169.

DeLisser, H. M., M. Christofidou-Solomidou, R. M. Strieter, M. D. Burdick, C. S. Robinson, R. S. Wexler, J. S. Kerr, C. Garlanda, J. R. Merwin, J. A. Madri, and S. M. Albelda. 1997. Involvement of endothelial PECAM-1/CD31 in angiogenesis. The American Journal of Pathology 151(3):671-677.

Denekamp, J. 1993. Review article: angiogenesis, neovascular proliferation and vascular pathophysiology as targets for cancer therapy. The British Journal of Radiology 66(783):181-196.

Dewald, G. W., S. R. Brockman, S. F. Paternoster, N. D. Bone, J. R. O’Fallon, C. Allmer, C. D. James, D. F. Jelinek, R. C. Tschumper, C. A. Hanson, R. K. Pruthi, T. E. Witzig, T. G. Call, and N. E. Kay. 2003. Chromosome anomalies detected by interphase fluorescence in situ hybridization: correlation with significant biological features of B-cell chronic lymphocytic leukaemia. British Journal of Haematology 121(2):287-295. 
Diao, L., and Y.-G. Chen. 2007. PTEN, a general negative regulator of cyclin D expression. Cell Research 17(4):291-292.

Dong, X.-F., Q. Song, L.-Z. Li, C.-L. Zhao, and L.-Q. Wang. 2007. Histone deacetylase inhibitor valproic acid inhibits proliferation and induces apoptosis in KM3 cells via downregulating VEGF receptor. Neuro Endocrinology Letters 28(6):775-780.

Dong, Q., P. Meng, T. Wang, W. Qin, W. Qin, F. Wang, J. Yuan, Z. Chen, A. Yang, and H. Wang. 2010. MicroRNA let-7a inhibits proliferation of human prostate cancer cells in vitro and in vivo by targeting E2F2 and CCND2. PloS One 5(4):e10147.

Don, R. H., P. T. Cox, B. J. Wainwright, K. Baker, and J. S. Mattick. 1991. "Touchdown" PCR to circumvent spurious priming during gene amplification. Nucleic Acids Research 19(14):4008.

Draisma, G., R. Etzioni, A. Tsodikov, A. Mariotto, E. Wever, R. Gulati, E. Feuer, and H. de Koning. 2009. Lead time and overdiagnosis in prostate-specific antigen screening: importance of methods and context. Journal of the National Cancer Institute 101(6):374383.

Drobnjak, M., I. Osman, H. I. Scher, M. Fazzari, and C. Cordon-Cardo. 2000. Overexpression of Cyclin D1 Is Associated with Metastatic Prostate Cancer to Bone. Clinical Cancer Research 6(5):1891-1895.

Duque, J. L., K. R. Loughlin, R. M. Adam, P. W. Kantoff, D. Zurakowski, and M. R. Freeman. 1999. Plasma levels of vascular endothelial growth factor are increased in patients with metastatic prostate cancer. Urology 54(3):523-527.

Du, Y., and J. R. Grandis. 2015. Receptor-type protein tyrosine phosphatases in cancer. Chinese Journal of Cancer 34(2):61-69.

Du, Z., C. Fujiyama, Y. Chen, and Z. Masaki. 2003. Expression of hypoxia-inducible factor 1alpha in human normal, benign, and malignant prostate tissue. Chinese Medical Journal 116(12):1936-1939.

Dvorak, H. F. 2002. Vascular permeability factor/vascular endothelial growth factor: a critical cytokine in tumor angiogenesis and a potential target for diagnosis and therapy. Journal of Clinical Oncology: Official Journal of the American Society of Clinical Oncology 20(21):4368-4380.

Edelstein, L. C., S. Micheva-Viteva, B. D. Phelan, and J. P. Dougherty. 2009. Short Communication: Activation of Latent HIV Type 1 Gene Expression by Suberoylanilide Hydroxamic Acid (SAHA), an HDAC Inhibitor Approved for Use to Treat Cutaneous T Cell Lymphoma. AIDS Research and Human Retroviruses 25(9):883-887.

El-Amm, J., and J. B. Aragon-Ching. 2015. Radium-223 for the treatment of castration-resistant prostate cancer. OncoTargets and therapy 8:1103-1109. 
El-Gohary, Y. M., J. F. Silverman, P. R. Olson, Y. L. Liu, J. K. Cohen, R. Miller, and R. S. Saad. 2007. Endoglin (CD105) and vascular endothelial growth factor as prognostic markers in prostatic adenocarcinoma. American Journal of Clinical Pathology 127(4):572-579.

Evron, E., C. B. Umbricht, D. Korz, V. Raman, D. M. Loeb, B. Niranjan, L. Buluwela, S. A. Weitzman, J. Marks, and S. Sukumar. 2001. Loss of cyclin D2 expression in the majority of breast cancers is associated with promoter hypermethylation. Cancer Research $61(6): 2782-2787$.

Ewen, M. E., and J. Lamb. 2004. The activities of cyclin D1 that drive tumorigenesis. Trends in Molecular Medicine 10(4):158-162.

Ewen, M. E., H. K. Sluss, C. J. Sherr, H. Matsushime, J. Kato, and D. M. Livingston. 1993. Functional interactions of the retinoblastoma protein with mammalian D-type cyclins. Cell 73(3):487-497.

Fedier, A., K. J. Dedes, P. Imesch, A. O. Von Bueren, and D. Fink. 2007. The histone deacetylase inhibitors suberoylanilide hydroxamic (Vorinostat) and valproic acid induce irreversible and MDR1-independent resistance in human colon cancer cells. International Journal of Oncology 31(3):633-641.

Ferrara, N. 1995. The role of vascular endothelial growth factor in pathological angiogenesis. Breast Cancer Research and Treatment 36(2):127-137.

Ferrara, N., and R. S. Kerbel. 2005. Angiogenesis as a therapeutic target. Nature 438(7070):967-974.

Fife, R. S., B. T. Rougraff, C. Proctor, and G. W. Sledge Jr. 1997. Inhibition of proliferation and induction of apoptosis by doxycycline in cultured human osteosarcoma cells. Journal of Laboratory and Clinical Medicine 130(5):530-534.

Fina, L., H. V. Molgaard, D. Robertson, N. J. Bradley, P. Monaghan, D. Delia, D. R. Sutherland, M. A. Baker, and M. F. Greaves. 1990. Expression of the CD34 gene in vascular endothelial cells. Blood 75(12):2417-2426.

Fischer, H., J. Chen, L. Skoog, and A. Lindblom. 2002. Cyclin D2 expression in familial and sporadic breast cancer. Oncology Reports 9(6):1157-1161.

Fischkoff, S. A., and E. Walter. 1984. Induction of neutrophilic differentiation of human promyelocytic leukemic cells by branched-chain carboxylic acid anticonvulsant drugs. Journal of Biological Response Modifiers 3(2):132-137.

Folkman, J. 1971. Tumor angiogenesis: therapeutic implications. The New England Journal of Medicine 285(21):1182-1186.

Folkman, J. 1974. Tumor angiogenesis. Advances in Cancer Research 19(0):331-358. 
Fonseca, R., B. Barlogie, R. Bataille, C. Bastard, P. L. Bergsagel, M. Chesi, F. E. Davies, J. Drach, P. R. Greipp, I. R. Kirsch, W. M. Kuehl, J. M. Hernandez, S. Minvielle, L. M. Pilarski, J. D. Shaughnessy, A. K. Stewart, and H. Avet-Loiseau. 2004. Genetics and cytogenetics of multiple myeloma: a workshop report. Cancer Research 64(4):15461558.

Fortunati, N., S. Bertino, L. Costantino, O. Bosco, I. Vercellinatto, M. G. Catalano, and G. Boccuzzi. 2008. Valproic acid is a selective antiproliferative agent in estrogen-sensitive breast cancer cells. Cancer Letters 259(2):156-164.

Fotiou, K., G. Vaiopoulos, K. Lilakos, A. Giannopoulos, K. Mandalenaki, G. Marinos, G. Koritsiadis, J. Sourdis, E. Konstantinidou, and K. Konstantopoulos. 2007. Serum ceruloplasmin as a marker in prostate cancer. Minerva Urologica $E$ Nefrologica $=$ The Italian Journal of Urology and Nephrology 59(4):407-411.

Fu, M., C. Wang, Z. Li, T. Sakamaki, and R. G. Pestell. 2004. Minireview: Cyclin D1: normal and abnormal functions. Endocrinology 145(12):5439-5447.

Gan, C. P., S. Hamid, S. Y. Hor, R. B. Zain, S. M. Ismail, W. M. Wan Mustafa, S. H. Teo, N. Saunders, and S. C. Cheong. 2012. Valproic acid: growth inhibition of head and neck cancer by induction of terminal differentiation and senescence. Head \& Neck 34(3):344353.

Ganguly, S. S., X. Li, and C. K. MiRNAanti. 2014. The Host Microenvironment Influences Prostate Cancer Invasion, Systemic Spread, Bone Colonization, and Osteoblastic Metastasis. Frontiers in Oncology 4.

Gao, D., Q. Xia, J. Lv, and H. Zhang. 2007. Chronic administration of valproic acid inhibits PC3 cell growth by suppressing tumor angiogenesis in vivo. International Journal of Urology: Official Journal of the Japanese Urological Association 14(9):838-845.

George, D. J., S. Halabi, T. F. Shepard, N. J. Vogelzang, D. F. Hayes, E. J. Small, P. W. Kantoff, and Cancer and Leukemia Group B 9480. 2001. Prognostic significance of plasma vascular endothelial growth factor levels in patients with hormone-refractory prostate cancer treated on Cancer and Leukemia Group B 9480. Clinical Cancer Research: An Official Journal of the American Association for Cancer Research 7(7):1932-1936.

Gillet, N., A. Florins, M. Boxus, C. Burteau, A. Nigro, F. Vandermeers, H. Balon, A.-B. Bouzar, J. Defoiche, A. Burny, M. Reichert, R. Kettmann, and L. Willems. 2007. Mechanisms of leukemogenesis induced by bovine leukemia virus: prospects for novel anti-retroviral therapies in human. Retrovirology 4:18.

Gillett, C., P. Smith, W. Gregory, M. Richards, R. Millis, G. Peters, and D. Barnes. 1996. Cyclin D1 and prognosis in human breast cancer. International Journal of Cancer. Journal International Du Cancer 69(2):92-99. 
Gingrich, J. R., R. J. Barrios, B. A. Foster, and N. M. Greenberg. 1999. Pathologic progression of autochthonous prostate cancer in the TRAMP model. Prostate Cancer and Prostatic Diseases 2(2):70-75.

Gingrich, J. R., R. J. Barrios, M. W. Kattan, H. S. Nahm, M. J. Finegold, and N. M. Greenberg. 1997. Androgen-independent prostate cancer progression in the TRAMP model. Cancer Research 57(21):4687-4691.

Gingrich, J. R., R. J. Barrios, R. A. Morton, B. F. Boyce, F. J. DeMayo, M. J. Finegold, R. Angelopoulou, J. M. Rosen, and N. M. Greenberg. 1996. Metastatic prostate cancer in a transgenic mouse. Cancer Research 56(18):4096-4102.

Göttlicher, M., S. Minucci, P. Zhu, O. H. Krämer, A. Schimpf, S. Giavara, J. P. Sleeman, F. Lo Coco, C. Nervi, P. G. Pelicci, and T. Heinzel. 2001. Valproic acid defines a novel class of HDAC inhibitors inducing differentiation of transformed cells. The EMBO Journal 20(24):6969-6978.

Greenberg, N. M., F. DeMayo, M. J. Finegold, D. Medina, W. D. Tilley, J. O. Aspinall, G. R. Cunha, A. A. Donjacour, R. J. Matusik, and J. M. Rosen. 1995. Prostate cancer in a transgenic mouse. Proceedings of the National Academy of Sciences of the United States of America 92(8):3439-3443.

Greenberg, N. M., F. J. DeMayo, P. C. Sheppard, R. Barrios, R. Lebovitz, M. Finegold, R. Angelopoulou, J. G. Dodd, M. L. Duckworth, and J. M. Rosen. 1994. The rat probasin gene promoter directs hormonally and developmentally regulated expression of a heterologous gene specifically to the prostate in transgenic mice. Molecular Endocrinology (Baltimore, Md.) 8(2):230-239.

Hanahan, D. 1983. Studies on transformation of Escherichia coli with plasmids. Journal of Molecular Biology 166(4):557-580.

Hanahan, D. and R. A. Weinberg. 2002. The hallmarks of cancer. Cell 100(1):57-70.

Hanahan, D. and R. A. Weinberg. 2011. Hallmarks of cancer: the next generation. Cell 144(5): 646-674.

Hansen, T. M., H. Singh, T. A. Tahir, and N. P. J. Brindle. 2010. Effects of angiopoietins-1 and 2 on the receptor tyrosine kinase Tie2 are differentially regulated at the endothelial cell surface. Cellular Signalling 22(3):527-532.

Hardenberg, S. 2010: Charakterisierung von Leupaxin und seiner Interaktionspartner in Karzinomzellen. Dissertation. University Göttingen, Germany. Institute for Human Genetics 
Harris, W. P., E. A. Mostaghel, P. S. Nelson and B. Montgomery. 2009. Androgen deprivation therapy: progress in understanding mechanisms of resistance and optimizing androgen depletion. Nature Clinical Practice Urology 6: 76-85.

Hecker, K. H., and K. H. Roux. 1996. High and low annealing temperatures increase both specificity and yield in touchdown and stepdown PCR. BioTechniques 20(3):478-485.

Henrique, R., V. L. Costa, N. Cerveira, A. L. Carvalho, M. O. Hoque, F. R. Ribeiro, J. Oliveira, M. R. Teixeira, D. Sidransky, and C. Jerónimo. 2006. Hypermethylation of Cyclin D2 is associated with loss of mRNA expression and tumor development in prostate cancer. Journal of Molecular Medicine (Berlin, Germany) 84(11):911-918.

Holcomb, I. N., J. M. Young, I. M. Coleman, K. Salari, D. I. Grove, L. Hsu, L. D. True, M. P. Roudier, C. M. Morrissey, C. S. Higano, P. S. Nelson, R. L. Vessella, and B. J. Trask. 2009. Comparative analyses of chromosome alterations in soft-tissue metastases within and across patients with castration-resistant prostate cancer. Cancer Research 69(19):7793-7802.

Holmgren, L., M. S. O’Reilly, and J. Folkman. 1995. Dormancy of micrometastases: Balanced proliferation and apoptosis in the presence of angiogenesis suppression. Nature Medicine 1(2):149-153.

Hong, Y.-K., N. Harvey, Y.-H. Noh, V. Schacht, S. Hirakawa, M. Detmar, and G. Oliver. 2002. Prox1 is a master control gene in the program specifying lymphatic endothelial cell fate. Developmental Dynamics: An Official Publication of the American Association of Anatomists 225(3):351-357.

Hoque, M. O., J.-C. Soria, J. Woo, T. Lee, J. Lee, S. J. Jang, S. Upadhyay, B. Trink, C. Monitto, C. Desmaze, L. Mao, D. Sidransky, and C. Moon. 2006. Aquaporin 1 Is Overexpressed in Lung Cancer and Stimulates NIH-3T3 Cell Proliferation and Anchorage-Independent Growth. The American Journal of Pathology 168(4):1345-1353.

Horoszewicz, J. S., S. S. Leong, E. Kawinski, J. P. Karr, H. Rosenthal, T. M. Chu, E. A. MiRNAand, and G. P. Murphy. 1983. LNCaP model of human prostatic carcinoma. Cancer Research 43(4):1809-1818.

Hsu, A., C. P. Wong, Z. Yu, D. E. Williams, R. H. Dashwood, and E. Ho. 2011. Promoter demethylation of cyclin D2 by sulforaphane in prostate cancer cells. Clinical Epigenetics 3(1):3.

Huang, W., H. Y. Chang, T. Fei, H. Wu, and Y.-G. Chen. 2007. GSK3 beta mediates suppression of cyclin D2 expression by tumor suppressor PTEN. Oncogene 26(17):2471-2482.

Huard, J. M., C. C. Forster, M. L. Carter, P. Sicinski, and M. E. Ross. 1999. Cerebellar histogenesis is disturbed in mice lacking cyclin D2. Development (Cambridge, England) 126(9):1927-1935. 
Hubaux, R., F. Vandermeers, M. C. Crisanti, C. Crisanti, V. Kapoor, A. Burny, C. Mascaux, S. M. Albelda, and L. Willems. 2010. Preclinical evidence for a beneficial impact of valproate on the response of small cell lung cancer to first-line chemotherapy. European Journal of Cancer (Oxford, England: 1990) 46(9):1724-1734.

Hu, B., and S.-Y. Cheng. 2009. Angiopoietin-2: Development of Inhibitors for Cancer Therapy. Current oncology reports 11(2):111-116.

Hu, G. 1993. DNA polymerase-catalyzed addition of nontemplated extra nucleotides to the 3' end of a DNA fragment. DNA and cell biology 12(8):763-770.

Hurwitz, H., L. Fehrenbacher, W. Novotny, T. Cartwright, J. Hainsworth, W. Heim, J. Berlin, A. Baron, S. Griffing, E. Holmgren, N. Ferrara, G. Fyfe, B. Rogers, R. Ross, and F. Kabbinavar. 2004. Bevacizumab plus irinotecan, fluorouracil, and leucovorin for metastatic colorectal cancer. The New England Journal of Medicine 350(23):2335-2342.

Hwang, C., and E. I. Heath. 2010. Angiogenesis inhibitors in the treatment of prostate cancer. Journal of Hematology \& Oncology 3(1):26.

Irshad, S., and C. Abate-Shen. 2013. Modeling prostate cancer in mice: Something old, something new, something premalignant, something metastatic. Cancer metastasis reviews 32(0):109-122.

Jha, G. G., V. Anand, A. Soubra, and B. R. Konety. 2014. Challenges of managing elderly men with prostate cancer. Nature Reviews Clinical Oncology 11(6):354-364.

Johannessen, C. U. 2000. Mechanisms of action of valproate: a commentatory. Neurochemistry International 37(2-3):103-110.

Johannessen, C. U., and S. I. Johannessen. 2003. Valproate: past, present, and future. CNS drug reviews 9(2):199-216.

Jones, J., W. Bentas, R. A. Blaheta, J. Makarevic, L. Hudak, S. Wedel, M. Probst, D. Jonas, and E. Juengel. 2008. Modulation of adhesion and growth of colon and pancreatic cancer cells by the histone deacetylase inhibitor valproic acid. International Journal of Molecular Medicine 22(3):293-299.

Jones, J., E. Juengel, A. Mickuckyte, L. Hudak, S. Wedel, D. Jonas, and R. A. Blaheta. 2009. The histone deacetylase inhibitor valproic acid alters growth properties of renal cell carcinoma in vitro and in vivo. Journal of Cellular and Molecular Medicine 13(8B):23762385.

Juengel, E., J. Makarević, I. Tsaur, G. Bartsch, K. Nelson, A. Haferkamp, and R. A. Blaheta. 2013. Resistance after chronic application of the HDAC-inhibitor valproic acid is associated with elevated Akt activation in renal cell carcinoma in vivo. PloS One 8(1):e53100. 
Kaighn, M. E., K. S. Narayan, Y. Ohnuki, J. F. Lechner, and L. W. Jones. 1979. Establishment and characterization of a human prostatic carcinoma cell line (PC-3). Investigative Urology 17(1):16-23.

Kantoff, P. W., C. S. Higano, N. D. Shore, E. R. Berger, E. J. Small, D. F. Penson, C. H. Redfern, A. C. Ferrari, R. Dreicer, R. B. Sims, Y. Xu, M. W. Frohlich, P. F. Schellhammer, and IMPACT Study Investigators. 2010. Sipuleucel-T immunotherapy for castration-resistant prostate cancer. The New England Journal of Medicine 363(5):411-422.

Karan, D., J. B. Thrasher, and D. Lubaroff. 2008. Prostate cancer: genes, environment, immunity and the use of immunotherapy. Prostate Cancer and Prostatic Diseases 11(3):230-236.

Kasper, S., P. C. Sheppard, Y. Yan, N. Pettigrew, A. D. Borowsky, G. S. Prins, J. G. Dodd, M. L. Duckworth, and R. J. Matusik. 1998. Development, progression, and androgendependence of prostate tumors in probasin-large T antigen transgenic mice: a model for prostate cancer. Laboratory Investigation; a Journal of Technical Methods and Pathology 78(6):i-xv.

Kaushik, D., V. Vashistha, S. Isharwal, S. A. Sediqe, and M.-F. Lin. 2015. Histone deacetylase inhibitors in castration-resistant prostate cancer: molecular mechanism of action and recent clinical trials. Therapeutic Advances in Urology 7(6):388-395.

Kellokumpu-Lehtinen, P., M. Talpaz, D. Harris, Q. Van, R. Kurzrock, and Z. Estrov. 1996. Leukemia-inhibitory factor stimulates breast, kidney and prostate cancer cell proliferation by paracrine and autocrine pathways. International Journal of Cancer. Journal International Du Cancer 66(4):515-519.

Kelly, W. K., S. Halabi, M. Carducci, D. George, J. F. Mahoney, W. M. Stadler, M. Morris, P. Kantoff, J. P. Monk, E. Kaplan, N. J. Vogelzang, and E. J. Small. 2012. Randomized, double-blind, placebo-controlled phase III trial comparing docetaxel and prednisone with or without bevacizumab in men with metastatic castration-resistant prostate cancer: CALGB 90401. Journal of Clinical Oncology: Official Journal of the American Society of Clinical Oncology 30(13):1534-1540.

Kluetz, P. G., W. D. Figg, and W. L. Dahut. 2010. Angiogenesis Inhibitors in the treatment of Prostate Cancer. Expert opinion on pharmacotherapy 11(2):233-247.

Kobayashi, T., E. Nakamura, Y. Shimizu, N. Terada, A. Maeno, G. Kobori, T. Kamba, T. Kamoto, O. Ogawa, and T. Inoue. 2009. Restoration of cyclin D2 has an inhibitory potential on the proliferation of LNCaP cells. Biochemical and Biophysical Research Communications 387(1):196-201.

Ko, E., Y. Kim, S.-E. Park, E. Y. Cho, J. Han, Y. M. Shim, J. Park, and D.-H. Kim. 2012. Reduced expression of cyclin D2 is associated with poor recurrence-free survival independent of cyclin D1 in stage III non-small cell lung cancer. Lung Cancer (Amsterdam, Netherlands) 77(2):401-406. 
Korbie, D. J., and J. S. Mattick. 2008. Touchdown PCR for increased specificity and sensitivity in PCR amplification. Nature Protocols 3(9):1452-1456.

Koren, G., and D. Kennedy. 1999. Safe use of valproic acid during pregnancy. Canadian Family Physician 45:1451-1453.

Koren, G., A. A. Nava-Ocampo, M. E. Moretti, R. Sussman, and I. Nulman. 2006. Major malformations with valproic acid. Canadian Family Physician 52(4):441-447.

Kostrouchová, M., Z. Kostrouch, and M. Kostrouchová. 2007. Valproic acid, a molecular lead to multiple regulatory pathways. Folia Biologica 53(2):37-49.

Kowalczyk, A., R. K. Filipkowski, M. Rylski, G. M. Wilczynski, F. A. Konopacki, J. Jaworski, M. A. Ciemerych, P. Sicinski, and L. Kaczmarek. 2004. The critical role of cyclin D2 in adult neurogenesis. The Journal of Cell Biology 167(2):209-213.

Koyama-Nasu, R., Y. Nasu-Nishimura, T. Todo, Y. Ino, N. Saito, H. Aburatani, K. Funato, K. Echizen, H. Sugano, R. Haruta, M. Matsui, R. Takahashi, E. Manabe, T. Oda, and T. Akiyama. 2013. The critical role of cyclin D2 in cell cycle progression and tumorigenicity of glioblastoma stem cells. Oncogene 32(33):3840-3845.

Kozar, K., M. A. Ciemerych, V. I. Rebel, H. Shigematsu, A. Zagozdzon, E. Sicinska, Y. Geng, Q. Yu, S. Bhattacharya, R. T. Bronson, K. Akashi, and P. Sicinski. 2004. Mouse development and cell proliferation in the absence of D-cyclins. Cell 118(4):477-491.

Kozar, K., and P. Sicinski. 2005. Cell cycle progression without cyclin D-CDK4 and cyclin DCDK6 complexes. Cell Cycle (Georgetown, Tex.) 4(3):388-391.

Krämer, O. H., P. Zhu, H. P. Ostendorff, M. Golebiewski, J. Tiefenbach, M. A. Peters, B. Brill, B. Groner, I. Bach, T. Heinzel, and M. Göttlicher. 2003. The histone deacetylase inhibitor valproic acid selectively induces proteasomal degradation of HDAC2. The EMBO Journal 22(13):3411-3420.

Krishnan, A. V., J. Moreno, L. Nonn, S. Swami, D. M. Peehl, and D. Feldman. 2007. Calcitriol as a chemopreventive and therapeutic agent in prostate cancer: role of anti-inflammatory activity. Journal of Bone and Mineral Research: The Official Journal of the American Society for Bone and Mineral Research 22 Suppl 2:V74-80.

Ladam, F., I. Damour, P. Dumont, Z. Kherrouche, Y. de Launoit, D. Tulasne, and A. ChotteauLelievre. 2013. Loss of a negative feedback loop involving pea3 and cyclin d2 is required for pea3-induced migration in transformed mammary epithelial cells. Molecular cancer research: MCR 11(11):1412-1424.

Laemmli, U. K. 1970. Cleavage of structural proteins during the assembly of the head of bacteriophage T4. Nature 227(5259):680-685. 
Lammer, E. J., L. E. Sever, and G. P. Oakley. 1987. Teratogen update: valproic acid. Teratology 35(3):465-473.

Langley, K. E., M. R. Villarejo, A. V. Fowler, P. J. Zamenhof, and I. Zabin. 1975. Molecular basis of beta-galactosidase alpha-complementation. Proceedings of the National Academy of Sciences of the United States of America 72(4):1254-1257.

Latil, A., P. Morant, G. Fournier, P. Mangin, P. Berthon, and O. Cussenot. 2002. CHC1-L, a candidate gene for prostate carcinogenesis at $13 q 14.2$, is frequently affected by loss of heterozygosity and underexpressed in human prostate cancer. International Journal of Cancer. Journal International Du Cancer 99(5):689-696.

Leclerc, D., L. Deng, J. Trasler, and R. Rozen. 2004. ApcMin/+ mouse model of colon cancer: gene expression profiling in tumors. Journal of Cellular Biochemistry 93(6):1242-1254.

Lee, R. J., and M. R. Smith. 2013. Targeting MET and VEGFR Signaling in Castration-Resistant Prostate Cancer. Cancer journal (Sudbury, Mass.) 19(1):90-98.

Lehn, S., N. P. Tobin, P. Berglund, K. Nilsson, A. H. Sims, K. Jirström, P. Härkönen, R. Lamb, and G. Landberg. 2010. Down-Regulation of the Oncogene Cyclin D1 Increases Migratory Capacity in Breast Cancer and Is Linked to Unfavorable Prognostic Features. The American Journal of Pathology 177(6):2886-2897.

Lei, N., Z. Song, B. Lu, Z. Tan, J. Pei, W. Liu, and K. Xu. 2014. Docetaxel-based therapy with and without antiangiogenic agents as first-line chemotherapy for castration-resistant prostate cancer: A meta-analysis of nine randomized controlled trials. Molecular and Clinical Oncology 2(6):1182-1188.

Leja, J., A. Essaghir, M. Essand, K. Wester, K. Öberg, T. H. Tötterman, R. Lloyd, G. Vasmatzis, J.-B. Demoulin, and V. Giandomenico. 2008. Novel markers for enterochromaffin cells and gastrointestinal neuroendocrine carcinomas. Modern Pathology 22(2):261-272.

Leung, D. W., G. Cachianes, W. J. Kuang, D. V. Goeddel, and N. Ferrara. 1989. Vascular endothelial growth factor is a secreted angiogenic mitogen. Science (New York, N.Y.) 246(4935):1306-1309.

Liu, A. Y., and L. D. True. 2002. Characterization of Prostate Cell Types by CD Cell Surface Molecules. The American Journal of Pathology 160(1):37-43.

Li, X. J., Z. J. Ren, and J. H. Tang. 2014. MicroRNA-34a: a potential therapeutic target in human cancer. Cell Death \& Disease 5(7):e1327.

Machado, M. C. C., M. Bellodi-Privato, M. S. Kubrusly, N. A. T. Molan, T. Tharcisio, E. R. de Oliveira, and L. A. C. D'Albuquerque. 2011. Valproic acid inhibits human hepatocellular cancer cells growth in vitro and in vivo. Journal of Experimental Therapeutics \& Oncology 9(2):85-92. 
Maisonpierre, P. C., C. Suri, P. F. Jones, S. Bartunkova, S. J. Wiegand, C. Radziejewski, D. Compton, J. McClain, T. H. Aldrich, N. Papadopoulos, T. J. Daly, S. Davis, T. N. Sato, and G. D. Yancopoulos. 1997. Angiopoietin-2, a natural antagonist for Tie2 that disrupts in vivo angiogenesis. Science (New York, N.Y.) 277(5322):55-60.

Mandriota, S. J., L. Jussila, M. Jeltsch, A. Compagni, D. Baetens, R. Prevo, S. Banerji, J. Huarte, R. Montesano, D. G. Jackson, L. Orci, K. Alitalo, G. Christofori, and M. S. Pepper. 2001. Vascular endothelial growth factor-C-mediated lymphangiogenesis promotes tumour metastasis. The EMBO Journal 20(4):672-682.

Marron, M. B., D. P. Hughes, M. D. Edge, C. L. Forder, and N. P. Brindle. 2000. Evidence for heterotypic interaction between the receptor tyrosine kinases TIE-1 and TIE-2. The Journal of Biological Chemistry 275(50):39741-39746.

Marron, M. B., H. Singh, T. A. Tahir, J. Kavumkal, H.-Z. Kim, G. Y. Koh, and N. P. J. Brindle. 2007. Regulated proteolytic processing of Tie1 modulates ligand responsiveness of the receptor-tyrosine kinase Tie2. The Journal of Biological Chemistry 282(42):30509_ 30517.

Martin, F., T. Linden, D. M. Katschinski, F. Oehme, I. Flamme, C. K. Mukhopadhyay, K. Eckhardt, J. Tröger, S. Barth, G. Camenisch, and R. H. Wenger. 2005. Copperdependent activation of hypoxia-inducible factor (HIF)-1: implications for ceruloplasmin regulation. Blood 105(12):4613-4619.

Matsubayashi, H., N. Sato, N. Fukushima, C. J. Yeo, K. M. Walter, K. Brune, F. Sahin, R. H. Hruban, and M. Goggins. 2003. Methylation of cyclin D2 is observed frequently in pancreatic cancer but is also an age-related phenomenon in gastrointestinal tissues. Clinical Cancer Research: An Official Journal of the American Association for Cancer Research 9(4):1446-1452.

Matsushime, H., M. E. Ewen, D. K. Strom, J. Y. Kato, S. K. Hanks, M. F. Roussel, and C. J. Sherr. 1992. Identification and properties of an atypical catalytic subunit (p34PSKJ3/cdk4) for mammalian D type G1 cyclins. Cell 71(2):323-334.

Matuo, Y., P. S. Adams, N. Nishi, H. Yasumitsu, J. W. Crabb, R. J. Matusik, and W. L. McKeehan. 1989. The androgen-dependent rat prostate protein, probasin, is a heparinbinding protein that co-purifies with heparin-binding growth factor-1. In Vitro Cellular \& Developmental Biology: Journal of the Tissue Culture Association 25(6):581-584.

McAnuff, M. A., G. R. Rettig, and K. G. Rice. 2007. Potency of siRNA versus shRNA mediated knockdown in vivo. Journal of Pharmaceutical Sciences 96(11):2922-2930.

Mentor-Marcel, R., C. A. Lamartiniere, I. E. Eltoum, N. M. Greenberg, and A. Elgavish. 2001. Genistein in the diet reduces the incidence of poorly differentiated prostatic adenocarcinoma in transgenic mice (TRAMP). Cancer Research 61(18):6777-6782. 
Mermelshtein, A., A. Gerson, S. Walfisch, B. Delgado, G. Shechter-Maor, J. Delgado, A. Fich, and L. Gheber. 2005. Expression of D-type cyclins in colon cancer and in cell lines from colon carcinomas. British Journal of Cancer 93(3):338-345.

Meyyappan, M., H. Wong, C. Hull, and K. T. Riabowol. 1998. Increased Expression of Cyclin D2 during Multiple States of Growth Arrest in Primary and Established Cells. Molecular and Cellular Biology 18(6):3163-3172.

Michaelis, M., U. R. Michaelis, I. Fleming, T. Suhan, J. Cinatl, R. A. Blaheta, K. Hoffmann, R. Kotchetkov, R. Busse, H. Nau, and J. Cinatl. 2004. Valproic acid inhibits angiogenesis in vitro and in vivo. Molecular Pharmacology 65(3):520-527.

Milde-Langosch, K., and S. Riethdorf. 2003. Role of cell-cycle regulatory proteins in gynecological cancer. Journal of Cellular Physiology 196(2):224-244.

Mizejewski, G. J. 1999. Role of integrins in cancer: survey of expression patterns. Proceedings of the Society for Experimental Biology and Medicine. Society for Experimental Biology and Medicine (New York, N.Y.) 222(2):124-138.

Montero, A. J., M. Escobar, G. Lopes, S. Glück, and C. Vogel. 2012. Bevacizumab in the Treatment of Metastatic Breast Cancer: Friend or Foe? Current Oncology Reports 14(1):1-11.

Motzer, R. J., T. E. Hutson, P. Tomczak, M. D. Michaelson, R. M. Bukowski, O. Rixe, S. Oudard, S. Negrier, C. Szczylik, S. T. Kim, I. Chen, P. W. Bycott, C. M. Baum, and R. A. Figlin. 2007. Sunitinib versus interferon alfa in metastatic renal-cell carcinoma. The New England Journal of Medicine 356(2):115-124.

Mukherji, D., S. Temraz, D. Wehbe, and A. Shamseddine. 2013. Angiogenesis and antiangiogenic therapy in prostate cancer. Critical Reviews in Oncology/Hematology $87(2): 122-131$.

Mullis, K. B., and F. A. Faloona. 1987. Specific synthesis of DNA in vitro via a polymerasecatalyzed chain reaction. Methods in Enzymology 155:335-350.

Munster, P., D. Marchion, E. Bicaku, M. Lacevic, J. Kim, B. Centeno, A. Daud, A. Neuger, S. Minton, and D. Sullivan. 2009. Clinical and biological effects of valproic acid as a histone deacetylase inhibitor on tumor and surrogate tissues: phase I/II trial of valproic acid and epirubicin/FEC. Clinical Cancer Research: An Official Journal of the American Association for Cancer Research 15(7):2488-2496.

Murphree, A. L., and W. F. Benedict. 1984. Retinoblastoma: clues to human oncogenesis. Science (New York, N.Y.) 223(4640):1028-1033.

Musgrove, E. A., C. E. Caldon, J. Barraclough, A. Stone, and R. L. Sutherland. 2011. Cyclin D as a therapeutic target in cancer. Nature Reviews Cancer 11(8):558-572. 
Muthukkaruppan, V. R., L. Kubai, and R. Auerbach. 1982. Tumor-induced neovascularization in the mouse eye. Journal of the National Cancer Institute 69(3):699-708.

Nayak, S. B., V. R. Bhat, D. Upadhyay, and S. L. Udupa. 2003. Copper and ceruloplasmin status in serum of prostate and colon cancer patients. Indian Journal of Physiology and Pharmacology 47(1):108-110.

Nelson, A. R., B. Fingleton, M. L. Rothenberg, and L. M. Matrisian. 2000. Matrix metalloproteinases: biologic activity and clinical implications. Journal of Clinical Oncology: Official Journal of the American Society of Clinical Oncology 18(5):11351149.

Neufeld, G., T. Cohen, S. Gengrinovitch, and Z. Poltorak. 1999. Vascular endothelial growth factor (VEGF) and its receptors. FASEB journal: official publication of the Federation of American Societies for Experimental Biology 13(1):9-22.

Nishida, N., H. Yano, T. Nishida, T. Kamura, and M. Kojiro. 2006. Angiogenesis in Cancer. Vascular Health and Risk Management 2(3):213-219.

Ohnuki, Y., M. M. Marnell, M. S. Babcock, J. F. Lechner, and M. E. Kaighn. 1980. Chromosomal analysis of human prostatic adenocarcinoma cell lines. Cancer Research 40(3):524-534.

Osuka, S., S. Takano, S. Watanabe, E. Ishikawa, T. Yamamoto, and A. Matsumura. 2012. Valproic acid inhibits angiogenesis in vitro and glioma angiogenesis in vivo in the brain. Neurologia Medico-Chirurgica 52(4):186-193.

Owens, M. J., and C. B. Nemeroff. 2003. Pharmacology of valproate. Psychopharmacology Bulletin 37 Suppl 2:17-24.

Ozawa, A., N. Tanji, T. Kikugawa, T. Sasaki, Y. Yanagihara, N. Miura, and M. Yokoyama. 2010. Inhibition of bladder tumour growth by histone deacetylase inhibitor. BJU international 105(8):1181-1186.

Padar, A., U. G. Sathyanarayana, M. Suzuki, R. Maruyama, J.-T. Hsieh, E. P. Frenkel, J. D. Minna, and A. F. Gazdar. 2003. Inactivation of cyclin D2 gene in prostate cancers by aberrant promoter methylation. Clinical Cancer Research: An Official Journal of the American Association for Cancer Research 9(13):4730-4734.

Pappa, C. A., G. Tsirakis, P. Kanellou, M. Kaparou, M. Stratinaki, A. Xekalou, A. Alegakis, A. Boula, E. N. Stathopoulos, and M. G. Alexandrakis. 2011. Monitoring serum levels ELR+ CXC chemokines and the relationship between microvessel density and angiogenic growth factors in multiple myeloma. Cytokine 56(3):616-620.

Parangi, S., M. O’Reilly, G. Christofori, L. Holmgren, J. Grosfeld, J. Folkman, and D. Hanahan. 1996. Antiangiogenic therapy of transgenic mice impairs de novo tumor growth. Proceedings of the National Academy of Sciences of the United States of America 93(5):2002-2007. 
Pass, H. I., G. J. Brewer, R. Dick, M. Carbone, and S. Merajver. 2008. A phase II trial of tetrathiomolybdate after surgery for malignant mesothelioma: final results. The Annals of Thoracic Surgery 86(2):383-389; discussion 390.

Pepper, M. S. 2001. Lymphangiogenesis and tumor metastasis: myth or reality? Clinical Cancer Research: An Official Journal of the American Association for Cancer Research $7(3): 462-468$.

Pfaffl, M. W. 2001. A new mathematical model for relative quantification in real-time RT-PCR. Nucleic Acids Research 29(9):e45.

Ponchel, F., C. Toomes, K. Bransfield, F. T. Leong, S. H. Douglas, S. L. Field, S. M. Bell, V. Combaret, A. Puisieux, A. J. Mighell, P. A. Robinson, C. F. Inglehearn, J. D. Isaacs, and A. F. Markham. 2003. Real-time PCR based on SYBR-Green I fluorescence: an alternative to the TaqMan assay for a relative quantification of gene rearrangements, gene amplifications and micro gene deletions. BMC biotechnology 3:18.

Radu, A., V. Neubauer, T. Akagi, H. Hanafusa, and M.-M. Georgescu. 2003. PTEN induces cell cycle arrest by decreasing the level and nuclear localization of cyclin D1. Molecular and Cellular Biology 23(17):6139-6149.

Rafii, S., and M. Skobe. 2003. Splitting vessels: Keeping lymph apart from blood. Nature Medicine 9(2):166-168.

Raju, K. S., G. Alessandri, M. Ziche, and P. M. Gullino. 1982. Ceruloplasmin, copper ions, and angiogenesis. Journal of the National Cancer Institute 69(5):1183-1188.

Rathkopf, D. E., M. J. Morris, J. J. Fox, D. C. Danila, S. F. Slovin, J. H. Hager, P. J. Rix, E. Chow Maneval, I. Chen, M. Gönen, M. Fleisher, S. M. Larson, C. L. Sawyers, and H. I. Scher. 2013. Phase I study of ARN-509, a novel antiandrogen, in the treatment of castrationresistant prostate cancer. Journal of Clinical Oncology: Official Journal of the American Society of Clinical Oncology 31(28):3525-3530.

Regan, C. M. 1985. Therapeutic levels of sodium valproate inhibit mitotic indices in cells of neural origin. Brain Research 347(2):394-398.

Robert Koch-Institute and German Centre for Cancer Registry Data (ZfKD). 2015. Krebs in Deutschland 2011/2012. 10th Edition, Berlin: Robert Koch-Institute.

Rocca, A., S. Minucci, G. Tosti, D. Croci, F. Contegno, M. Ballarini, F. Nolè, E. Munzone, A. Salmaggi, A. Goldhirsch, P. G. Pelicci, and A. Testori. 2009. A phase I-Il study of the histone deacetylase inhibitor valproic acid plus chemoimmunotherapy in patients with advanced melanoma. British Journal of Cancer 100(1):28-36. 
Rosenbaum, E., M. O. Hoque, Y. Cohen, M. Zahurak, M. A. Eisenberger, J. I. Epstein, A. W. Partin, and D. Sidransky. 2005. Promoter hypermethylation as an independent prognostic factor for relapse in patients with prostate cancer following radical prostatectomy. Clinical Cancer Research: An Official Journal of the American Association for Cancer Research 11(23):8321-8325.

Pichler, R. W. H. 2015. Bevacizumab as first-line therapy in metastatic renal cell carcinoma: Progression-free survival for 3 years. Der Urologe. Ausg. A.

Saiki, R. K., S. Scharf, F. Faloona, K. B. Mullis, G. T. Horn, H. A. Erlich, and N. Arnheim. 1985. Enzymatic amplification of beta-globin genomic sequences and restriction site analysis for diagnosis of sickle cell anemia. Science (New York, N.Y.) 230(4732):1350-1354.

Sambrook, J., E. F Fritsch, T. Maniatis. 1989. Molecular cloning: a laboratory manual. New York, USA, Cold Spring Habour.

Sami, S., N. Höti, H.-M. Xu, Z. Shen, and X. Huang. 2008. Valproic acid inhibits the growth of cervical cancer both in vitro and in vivo. Journal of Biochemistry 144(3):357-362.

Sandler, A., R. Gray, M. C. Perry, J. Brahmer, J. H. Schiller, A. Dowlati, R. Lilenbaum, and D. H. Johnson. 2006. Paclitaxel-carboplatin alone or with bevacizumab for non-small-cell lung cancer. The New England Journal of Medicine 355(24):2542-2550.

Sanger, F., and A. R. Coulson. 1975. A rapid method for determining sequences in DNA by primed synthesis with DNA polymerase. Journal of Molecular Biology 94(3):441-448.

Santarius, T., J. Shipley, D. Brewer, M. R. Stratton, and C. S. Cooper. 2010. A census of amplified and overexpressed human cancer genes. Nature Reviews Cancer 10(1):5964.

Schaefer, A., M. Jung, H.-J. Mollenkopf, I. Wagner, C. Stephan, F. Jentzmik, K. Miller, M. Lein, G. Kristiansen, and K. Jung. 2010. Diagnostic and prognostic implications of microRNA profiling in prostate carcinoma. International Journal of Cancer. Journal International Du Cancer 126(5):1166-1176.

Scher, H. I., K. Fizazi, F. Saad, M.-E. Taplin, C. N. Sternberg, K. Miller, R. de Wit, P. Mulders, K. N. Chi, N. D. Shore, A. J. Armstrong, T. W. Flaig, A. Fléchon, P. Mainwaring, M. Fleming, J. D. Hainsworth, M. Hirmand, B. Selby, L. Seely, J. S. de Bono, and AFFIRM Investigators. 2012. Increased survival with enzalutamide in prostate cancer after chemotherapy. The New England Journal of Medicine 367(13):1187-1197.

Shabbeer, S., M. S. Q. Kortenhorst, S. Kachhap, N. Galloway, R. Rodriguez, and M. A. Carducci. 2007. Multiple Molecular pathways explain the anti-proliferative effect of valproic acid on prostate cancer cells in vitro and in vivo. The Prostate 67(10):1099-1110.

Sharma, S. B., and J. M. Ruppert. 2015. MicroRNA-Based Therapeutic Strategies for Targeting Mutant and Wild Type RAS in Cancer. Drug Development Research. 
Sharma, S., J. Symanowski, B. Wong, P. Dino, P. Manno, and N. Vogelzang. 2008. A Phase II Clinical Trial of Oral Valproic Acid in Patients with Castration-Resistant Prostate Cancers Using an Intensive Biomarker Sampling Strategy. Translational Oncology 1(3):141-147.

Shen, M. M., and C. Abate-Shen. 2007. Pten inactivation and the emergence of androgenindependent prostate cancer. Cancer Research 67(14):6535-6538.

Shen, M. M., and C. Abate-Shen. 2010. Molecular genetics of prostate cancer: new prospects for old challenges. Genes \& Development 24(18):1967-2000.

Sicinski, P., J. L. Donaher, Y. Geng, S. B. Parker, H. Gardner, M. Y. Park, R. L. Robker, J. S. Richards, L. K. McGinnis, J. D. Biggers, J. J. Eppig, R. T. Bronson, S. J. Elledge, and R. A. Weinberg. 1996. Cyclin D2 is an FSH-responsive gene involved in gonadal cell proliferation and oncogenesis. Nature 384(6608):470-474.

Sidana, A., M. Wang, S. Shabbeer, W. H. Chowdhury, G. Netto, S. E. Lupold, M. Carducci, and R. Rodriguez. 2012. Mechanism of Growth Inhibition of Prostate Cancer Xenografts by Valproic Acid. Journal of Biomedicine and Biotechnology 2012.

Smith, D. C., M. R. Smith, C. Sweeney, A. A. Elfiky, C. Logothetis, P. G. Corn, N. J. Vogelzang, E. J. Small, A. L. Harzstark, M. S. Gordon, U. N. Vaishampayan, N. B. Haas, A. I. Spira, P. N. Lara, C.-C. Lin, S. Srinivas, A. Sella, P. Schöffski, C. Scheffold, A. L. Weitzman, and M. Hussain. 2013. Cabozantinib in patients with advanced prostate cancer: results of a phase II randomized discontinuation trial. Journal of Clinical Oncology: Official Journal of the American Society of Clinical Oncology 31(4):412-419.

Song, M. S., L. Salmena, and P. P. Pandolfi. 2012. The functions and regulation of the PTEN tumour suppressor. Nature Reviews. Molecular Cell Biology 13(5):283-296.

Song, Y., R. S. Maul, C. S. Gerbin, and D. D. Chang. 2002. Inhibition of Anchorage-independent Growth of Transformed NIH3T3 Cells by Epithelial Protein Lost in Neoplasm (EPLIN) Requires Localization of EPLIN to Actin Cytoskeleton. Molecular Biology of the Cell 13(4):1408-1416.

Spillane, D. R., D. Y. Wang, S. Newbigging, Y. Wang, C.-X. Shi, H.-R. Cho, H. Shimizu, A. Gramolini, M. Liu, and X.-Y. Wen. 2015. Chromosome Condensation 1-Like (Chc1L) Is a Novel Tumor Suppressor Involved in Development of Histiocyte-Rich Neoplasms. PLOS ONE 10(8).

Stettner, M., G. Krämer, A. Strauss, T. Kvitkina, S. Ohle, B. C. Kieseier, and P. Thelen. 2012. Long-term antiepileptic treatment with histone deacetylase inhibitors may reduce the risk of prostate cancer. European journal of cancer prevention: the official journal of the European Cancer Prevention Organisation (ECP) 21(1):55-64.

Stone, K. R., D. D. Mickey, H. Wunderli, G. H. Mickey, and D. F. Paulson. 1978. Isolation of a human prostate carcinoma cell line (DU 145). International Journal of Cancer. Journal International Du Cancer 21(3):274-281. 
Strieter, R. M., P. J. Polverini, S. L. Kunkel, D. A. Arenberg, M. D. Burdick, J. Kasper, J. Dzuiba, J. Van Damme, A. Walz, and D. Marriott. 1995. The functional role of the ELR motif in CXC chemokine-mediated angiogenesis. The Journal of Biological Chemistry 270(45):27348-27357.

Strohmeyer, D., C. Rössing, A. Bauerfeind, O. Kaufmann, H. Schlechte, G. Bartsch, and S. Loening. 2000. Vascular endothelial growth factor and its correlation with angiogenesis and p53 expression in prostate cancer. The Prostate 45(3):216-224.

Sun, L. and D. H. Coy. 2014. Anti-convulsant drug valproic acid in cancers and in combination anti-cancer therapeutics. Modern Chemistry and Applications, 2:118.

Surget, S., M. P. Khoury, and J.-C. Bourdon. 2013. Uncovering the role of p53 splice variants in human malignancy: a clinical perspective. OncoTargets and therapy 7:57-68.

Suri, C., P. F. Jones, S. Patan, S. Bartunkova, P. C. Maisonpierre, S. Davis, T. N. Sato, and G. D. Yancopoulos. 1996. Requisite role of angiopoietin-1, a ligand for the TIE2 receptor, during embryonic angiogenesis. Cell 87(7):1171-1180.

Takano, Y., Y. Kato, P. J. van Diest, M. Masuda, H. Mitomi, and I. Okayasu. 2000. Cyclin D2 overexpression and lack of p27 correlate positively and cyclin E inversely with a poor prognosis in gastric cancer cases. The American Journal of Pathology 156(2):585-594.

Tani, Y., A. Suttie, G. P. Flake, A. Nyska, and R. R. Maronpot. 2005. Epithelial-stromal tumor of the seminal vesicles in the transgenic adenocarcinoma mouse prostate model. Veterinary Pathology 42(3):306-314.

Tournaire, R., M.-P. Simon, F. le Noble, A. Eichmann, P. England, and J. Pouysségur. 2004. A short synthetic peptide inhibits signal transduction, migration and angiogenesis mediated by Tie2 receptor. EMBO reports 5(3):262-267.

Trotman, L. C., M. Niki, Z. A. Dotan, J. A. Koutcher, A. Di Cristofano, A. Xiao, A. S. Khoo, P. Roy-Burman, N. M. Greenberg, T. V. Dyke, C. Cordon-Cardo, and P. P. Pandolfi. 2003. Pten Dose Dictates Cancer Progression in the Prostate. PLoS Biol 1(3):e59.

Ummanni, R., E. Jost, M. Braig, F. Lohmann, F. Mundt, C. Barett, T. Schlomm, G. Sauter, T. Senff, C. Bokemeyer, H. Sültmann, C. Meyer-Schwesinger, T. H. Brümmendorf, and S. Balabanov. 2011. Ubiquitin carboxyl-terminal hydrolase 1 (UCHL1) is a potential tumour suppressor in prostate cancer and is frequently silenced by promoter methylation. Molecular Cancer 10:129.

Vallo, S., W. Xi, L. Hudak, E. Juengel, I. Tsaur, C. Wiesner, A. Haferkamp, and R. A. Blaheta. 2011. HDAC inhibition delays cell cycle progression of human bladder cancer cells in vitro. Anti-Cancer Drugs 22(10):1002-1009. 
Venkataramani, V., C. Rossner, L. Iffland, S. Schweyer, I. Y. Tamboli, J. Walter, O. Wirths, and T. A. Bayer. 2010. Histone deacetylase inhibitor valproic acid inhibits cancer cell proliferation via down-regulation of the alzheimer amyloid precursor protein. The Journal of Biological Chemistry 285(14):10678-10689.

Vieira, J., and J. Messing. 1982. The pUC plasmids, an M13mp7-derived system for insertion mutagenesis and sequencing with synthetic universal primers. Gene 19(3):259-268.

Virmani, A., A. Rathi, S. Heda, K. Sugio, C. Lewis, V. Tonk, T. Takahashi, J. A. Roth, J. D. Minna, D. M. Euhus, and A. F. Gazdar. 2003. Aberrant methylation of the cyclin D2 promoter in primary small cell, nonsmall cell lung and breast cancers. International Journal of Cancer. Journal International Du Cancer 107(3):341-345.

Voorzanger-Rousselot, N., P. Garnero, N. Voorzanger-Rousselot, P. Garnero, N. VoorzangerRousselot, and P. Garnero. 2007. Biochemical markers in oncology. Part I: Molecular basis. Part II: Clinical uses. Cancer Treatment Reviews 33(3):230-283.

Wagner, J. M.; G. Bug, M. Jung. 2010: Valproinsäure als Histon-Deacetylase-Hemmstoff. Neues Einsatzgebiet für einen altbekannten Arzneistoff. Pharmazie in unserer Zeit 39 (3):197-203.

Wagner, D., D. Trudel, T. Van der Kwast, L. Nonn, A. A. Giangreco, D. Li, A. Dias, M. Cardoza, S. Laszlo, K. Hersey, L. Klotz, A. Finelli, N. Fleshner, and R. Vieth. 2013a. Randomized clinical trial of vitamin D3 doses on prostatic vitamin D metabolite levels and ki67 labeling in prostate cancer patients. The Journal of Clinical Endocrinology and Metabolism 98(4):1498-1507.

Wagner, D., D. Trudel, T. Van der Kwast, L. Nonn, A. A. Giangreco, D. Li, A. Dias, M. Cardoza, S. Laszlo, K. Hersey, L. Klotz, A. Finelli, N. Fleshner, and R. Vieth. 2013b. Randomized clinical trial of vitamin D3 doses on prostatic vitamin D metabolite levels and ki67 labeling in prostate cancer patients. The Journal of Clinical Endocrinology and Metabolism 98(4):1498-1507.

Walter, B. A., V. A. Valera, P. A. Pinto, and M. J. Merino. 2013. Comprehensive microRNA Profiling of Prostate Cancer. Journal of Cancer 4(5):350-357.

Wang, S., J. Gao, Q. Lei, N. Rozengurt, C. Pritchard, J. Jiao, G. V. Thomas, G. Li, P. RoyBurman, P. S. Nelson, X. Liu, and H. Wu. 2003. Prostate-specific deletion of the murine Pten tumor suppressor gene leads to metastatic prostate cancer. Cancer Cell 4(3):209221.

Wedel, S., L. Hudak, J.-M. Seibel, J. Makarević, E. Juengel, I. Tsaur, A. Waaga-Gasser, A. Haferkamp, and R. A. Blaheta. 2011. Molecular targeting of prostate cancer cells by a triple drug combination down-regulates integrin driven adhesion processes, delays cell cycle progression and interferes with the cdk-cyclin axis. BMC cancer 11:375. 
Weidner, N., P. R. Carroll, J. Flax, W. Blumenfeld, and J. Folkman. 1993. Tumor angiogenesis correlates with metastasis in invasive prostate carcinoma. The American Journal of Pathology 143(2):401-409.

Weinberg, R. A. 1995. The retinoblastoma protein and cell cycle control. Cell 81(3):323-330.

Weller, M., T. Gorlia, J. G. Cairncross, M. J. van den Bent, W. Mason, K. Belanger, A. A. Brandes, U. Bogdahn, D. R. Macdonald, P. Forsyth, A. O. Rossetti, D. Lacombe, R.-O. MiRNAimanoff, C. J. Vecht, and R. Stupp. 2011. Prolonged survival with valproic acid use in the EORTC/NCIC temozolomide trial for glioblastoma. Neurology 77(12):11561164.

Wianny, F., F. X. Real, C. L. Mummery, M. Van Rooijen, J. Lahti, J. Samarut, and P. Savatier. 1998. G1-phase regulators, cyclin D1, cyclin D2, and cyclin D3: up-regulation at gastrulation and dynamic expression during neurulation. Developmental Dynamics: An Official Publication of the American Association of Anatomists 212(1):49-62.

Wigle, J. T., N. Harvey, M. Detmar, I. Lagutina, G. Grosveld, M. D. Gunn, D. G. Jackson, and G. Oliver. 2002. An essential role for Prox1 in the induction of the lymphatic endothelial cell phenotype. The EMBO journal 21(7):1505-1513.

Wilting, J., M. Papoutsi, B. Christ, K. H. Nicolaides, C. S. von Kaisenberg, J. Borges, G. B. Stark, K. Alitalo, S. I. Tomarev, C. Niemeyer, and J. Rössler. 2002. The transcription factor Prox1 is a marker for lymphatic endothelial cells in normal and diseased human tissues. FASEB journal: official publication of the Federation of American Societies for Experimental Biology 16(10):1271-1273.

Witt, D. 2009. Der Einfluss des Phytoöstrogens Tectorigenin und des HistondeacetylaseInhibitors Valproinsäure auf Wachstumsfaktor regulierte Signalwege im Prostatakarzinom. Diploma thesis. University Göttingen, Germany. Institute for Human Genetics.

Witt, D. 2012. Analyse zur Rolle von pflanzlichen Wirkstoffen und HistondeacetylaseInhibitoren auf Wachstumsfaktoren und deren Signalwege in Prostatakarzinomzellen. Dissertation. University Göttingen, Germany. Institute for Human Genetics.

Witt, D., P. Burfeind, S. von Hardenberg, L. Opitz, G. Salinas-Riester, F. Bremmer, S. Schweyer, P. Thelen, J. Neesen, and S. Kaulfuss. 2013. Valproic acid inhibits the proliferation of cancer cells by re-expressing cyclin D2. Carcinogenesis 34(5):1115-1124.

Wong, C. P., A. Hsu, A. Buchanan, Z. Palomera-Sanchez, L. M. Beaver, E. A. Houseman, D. E. Williams, R. H. Dashwood, and E. Ho. 2014. Effects of sulforaphane and 3,3'diindolylmethane on genome-wide promoter methylation in normal prostate epithelial cells and prostate cancer cells. PloS One 9(1):e86787.

Woollard, D. J., K. Opeskin, S. Coso, D. Wu, M. E. Baldwin, and E. D. Williams. 2013. Differential expression of VEGF ligands and receptors in prostate cancer. The Prostate 73(6):563572. 
Wu, X., J. Wu, J. Huang, W. C. Powell, J. Zhang, R. J. Matusik, F. O. Sangiorgi, R. E. Maxson, H. M. Sucov, and P. Roy-Burman. 2001. Generation of a prostate epithelial cell-specific Cre transgenic mouse model for tissue-specific gene ablation. Mechanisms of Development 101(1-2):61-69.

Xia, Q., J. Sung, W. Chowdhury, C.-L. Chen, N. Höti, S. Shabbeer, M. Carducci, and R. Rodriguez. 2006. Chronic administration of valproic acid inhibits prostate cancer cell growth in vitro and in vivo. Cancer Research 66(14):7237-7244.

Yamamura, S., S. Saini, S. Majid, H. Hirata, K. Ueno, G. Deng, and R. Dahiya. 2012. MicroRNA$34 a$ modulates c-Myc transcriptional complexes to suppress malignancy in human prostate cancer cells. PloS One 7(1):e29722.

Yeh, I.-T., R. L. Reddick, and A. P. Kumar. 2009. Malignancy arising in seminal vesicles in the transgenic adenocarcinoma of mouse prostate (TRAMP) model. The Prostate 69(7):755-760.

Yoshimoto, M., J.-C. Cutz, P. A. S. Nuin, A. M. Joshua, J. Bayani, A. J. Evans, M. Zielenska, and J. A. Squire. 2006. Interphase FISH analysis of PTEN in histologic sections shows genomic deletions in $68 \%$ of primary prostate cancer and $23 \%$ of high-grade prostatic intra-epithelial neoplasias. Cancer Genetics and Cytogenetics 169(2):128-137.

Yu, J., W. K. Leung, M. P. A. Ebert, R. W. L. Leong, P. C. H. Tse, M. W. Y. Chan, A. H. C. Bai, K. F. To, P. Malfertheiner, and J. J. Y. Sung. 2003. Absence of cyclin D2 expression is associated with promoter hypermethylation in gastric cancer. British Journal of Cancer 88(10):1560-1565.

Zhang, J., T. Z. Thomas, S. Kasper, and R. J. Matusik. 2000. A small composite probasin promoter confers high levels of prostate-specific gene expression through regulation by androgens and glucocorticoids in vitro and in vivo. Endocrinology 141(12):4698-4710.

Zhang, Z.-H., C.-L. Hao, P. Liu, X. Tian, L.-H. Wang, L. Zhao, and C.-M. Zhu. 2014. Valproic acid inhibits tumor angiogenesis in mice transplanted with Kasumi-1 leukemia cells. Molecular Medicine Reports 9(2):443-449.

Zhou, Y., Y. Xu, H. Wang, J. Niu, H. Hou, and Y. Jiang. 2014. Histone deacetylase inhibitor, valproic acid, radiosensitizes the C6 glioma cell line in vitro. Oncology Letters 7(1):203208.

Zhou, Z., A. Flesken-Nikitin, D. C. Corney, W. Wang, D. W. Goodrich, P. Roy-Burman, and A. Y. Nikitin. 2006. Synergy of p53 and Rb deficiency in a conditional mouse model for metastatic prostate cancer. Cancer Research 66(16):7889-7898.

Zhu, C., P. Shao, M. Bao, P. Li, H. Zhou, H. Cai, Q. Cao, L. Tao, X. Meng, X. Ju, C. Qin, J. Li, and C. Yin. 2014. miRNA-154 inhibits prostate cancer cell proliferation by targeting CCND2. Urologic Oncology 32(1):31.e9-16. 


\section{Acknowledgements}

I would like to express my gratitude to Prof. Dr. Peter Burfeind for being my referee, for his invaluable help and advice during this project and for proofreading of the thesis.

I would like to extend my gratitude to Dr. Silke Kaulfuß for giving me the opportunity to work on this interesting project and for the supervision. Especially I want to thank her for the endless support and encouragement, for her guidance and for proofreading this thesis.

I sincerely thank Prof. Dr. Matthias Dobbelstein and Prof. Dr. Dieter Kube for being my thesis committee members.

I am very thankful to Prof. Dr. Jörg Wilting for the great collaboration regarding the CAM assays. I also want to thank his study group, especially Kerstin, Sonja, Jürgen and Berti for their help and support.

I would like to thank all the members of the animal house and especially Lea for doing a great job taking care of the mice I worked with.

Special thanks to my lab colleagues Rabea, Julia, Rovena, Lisa, Jasmin, Sascha and Daria for the friendly atmosphere in the lab, their sympathetic ear, advice and for their generous help. Thank you Rabea for helping me windowing the eggs.

I also would like to thank my students Maike, Tashina and Tabea for their support in conducting this project.

I would like to thank all my institute colleagues for their support, advice and friendly atmosphere. Special thanks to Lukasz for the technical assistance.

My deepest gratitude is addressed to my family and Christian. Your constant support and love contributed tremendously to the completion of this work. Thank you Christian not only for all the help at work but also for always backing me up, putting faith in me, encouraging me and cheering me up, especially during the hard times of my study. Thank you Mom, Dad, Grandma, Martina and Dennis for always being there for me and supporting me in every possible way. I dedicate this work to my parents. 


\section{Curriculum Vitae}

Personal information:

$\begin{array}{ll}\text { Name: } & \text { Claudia Morich } \\ \text { Date of birth: } & 12.04 .1984 \\ \text { Place of birth: } & \text { Osterode am Harz } \\ \text { Nationality: } & \text { German }\end{array}$

Course of education:

1990-1994

Elementary school, Walkenried

1994-2013

Internatsgymnasium Pädagogium, Bad Sachsa, Graduation: A level

2006-2009

Studies in Biology (B.Sc.), Georg-August University, Göttingen, Graduation: Bachelor of Science

2009-2012

Studies in Developmental, Neural and Behavioral Biology (M.Sc.), Georg-August University, Göttingen, Graduation: Master of Science

2012-2016

Experimental studies for the doctoral thesis in the Institute of Human Genetics at the Georg-August University, Göttingen 